\title{
Strategies for non-invasive management of high-grade cervical intraepithelial neoplasia
}

Citation for published version (APA):

Koeneman, M. M. (2019). Strategies for non-invasive management of high-grade cervical intraepithelial neoplasia: prognostic biomarkers and immunotherapy. [Doctoral Thesis, Maastricht University]. Maastricht University. https://doi.org/10.26481/dis.20190116mk

Document status and date:

Published: 01/01/2019

DOI:

10.26481/dis.20190116mk

Document Version:

Publisher's PDF, also known as Version of record

\section{Please check the document version of this publication:}

- A submitted manuscript is the version of the article upon submission and before peer-review. There can be important differences between the submitted version and the official published version of record.

People interested in the research are advised to contact the author for the final version of the publication, or visit the DOI to the publisher's website.

- The final author version and the galley proof are versions of the publication after peer review.

- The final published version features the final layout of the paper including the volume, issue and page numbers.

Link to publication

\footnotetext{
General rights rights.

- You may freely distribute the URL identifying the publication in the public portal. please follow below link for the End User Agreement:

www.umlib.nl/taverne-license

Take down policy

If you believe that this document breaches copyright please contact us at:

repository@maastrichtuniversity.nl

providing details and we will investigate your claim.
}

Copyright and moral rights for the publications made accessible in the public portal are retained by the authors and/or other copyright owners and it is a condition of accessing publications that users recognise and abide by the legal requirements associated with these

- Users may download and print one copy of any publication from the public portal for the purpose of private study or research.

- You may not further distribute the material or use it for any profit-making activity or commercial gain

If the publication is distributed under the terms of Article $25 \mathrm{fa}$ of the Dutch Copyright Act, indicated by the "Taverne" license above, 



\section{Strategies for non-invasive management of high-grade cervical intraepithelial neoplasia: prognostic biomarkers and immunotherapy}

Margot Maria Koeneman 
(c) Copyright, M.M. Koeneman, Maastricht 2018

ISBN 978-94-6332-435-9

Graphic design by Loes Kema

Printing by GVO drukkers \& vormgevers, Ede, NL

All rights reserved. No part of this publication may be reproduced, stored in a retrieval system of any nature, or transmitted, in any form or by any means, electronic, mechanical, photocopying, recording or otherwise, without the prior permission in writing from the copyright owner.

Financial support for the publication of this thesis was provided by Stichting Olijf and Chipsoft. 


\section{Strategies for non-invasive management of high-grade cervical intraepithelial neoplasia: prognostic biomarkers and immunotherapy}

PROEFSCHRIFT

ter verkrijging van de graad van doctor aan de Universiteit Maastricht, op gezag van de Rector Magnificus, Prof. dr. Rianne M Letschert, volgens het besluit van het College van Decanen, in het openbaar te verdedigen op woensdag 16 januari 2019 om 14:00 uur

door

Margot Maria Koeneman geboren op 3 juni 1986 te Eindhoven 


\section{Promotores}

Prof. dr. RFPM Kruitwagen

Prof. dr. HW Nijman (Universitair Medisch Centrum Croningen)

\section{Copromotor}

Dr. A] Kruse

\section{Beoordelingscommissie}

Prof. dr. FCS Ramaekers (voorzitter)

Dr. MIE van de Poelgeest (Leids Universitair Medisch Centrum)

Prof. dr. EJM Speel

Prof. dr. KK van de Vijver (UZ Gent)

Prof. dr. GDEM van der Weijden 

Table of contents

Chapter1 Introduction 9

Part $1 \quad$ Assessment of clinical and molecular biomarkers as predictors for spontaneous regression of high-grade CIN lesions

Chapter $2 \quad$ Natural history of high-grade cervical intraepithelial neoplasia:a review of prognostic biomarkers Expert Review of Molecular Diagnostics. 2015 Apr;15(4):527-46

Chapter 3 A common epitope in HLA-DRB1*13/14 may protect against HPV16 related high-grade cervical intraepithelial neoplasia Submitted

Chapter 4 Gain of chromosomal region 3926 as a prognostic biomarker 79 for high-grade cervical intraepithelial neoplasia: literature overview and pilot study Pathology \& Oncology Research. 2018 Oct 25

Chapter 5 A prediction model for spontaneous regression of cervical intraepithelial neoplasia grade 2 , based on simple clinical parameters

Human Pathology. 2017]an;59:62-69

Chapter $6 \quad$ Smoking status and parity are associated with spontaneous 113 regression of high-risk HPV-positive $\mathrm{CIN}_{2}$ Submitted 
Part 2 Assessment of clinical applicability of imiquimod cream as a treatment modality for high-grade CIN

Chapter 7 Physicians' awareness, attitudes and experiences regarding 129 imiquimod treatment of vaginal and cervical intraepithelial neoplasia

Journal of Lower Cenital Tract Disease. 2016 Jan;20(1):75-9

Chapter 8 Treatment of cervical intraepithelial neoplasia: patients 151 preferences for surgery or immunotherapy with imiquimod Journal of Immunotherapy. 2017; 40:148-153

Chapter 9a TOPical Imiquimod treatment of high-grade Cervical 165 intraepithelial neoplasia (TOPIC trial): study protocol for a randomized controlled trial BMC Cancer. 2016 Feb 20;16:132

Chapter 9b Preliminary stop of the TOPical Imiquimod treatment of high- 179 grade Cervical intraepithelial neoplasia (TOPIC) trial.

BMC Cancer. 2017 Feb 7:17(1):110

$\begin{array}{lll}\text { Chapter10 Discussion } & 185\end{array}$

$\begin{array}{lll}\text { Chapter11 Valorisation } & 203\end{array}$

$\begin{array}{lll}\text { Chapter12 Summary } & 211\end{array}$

$\begin{array}{lll}\text { Chapter13 Nederlandstalige samenvatting } & 219\end{array}$

$\begin{array}{ll}\text { Abbreviations } & 227\end{array}$

Publications, presentations and awards $\quad 231$

$\begin{array}{ll}\text { Dankwoord } & 237\end{array}$

Curriculum vitae 243 


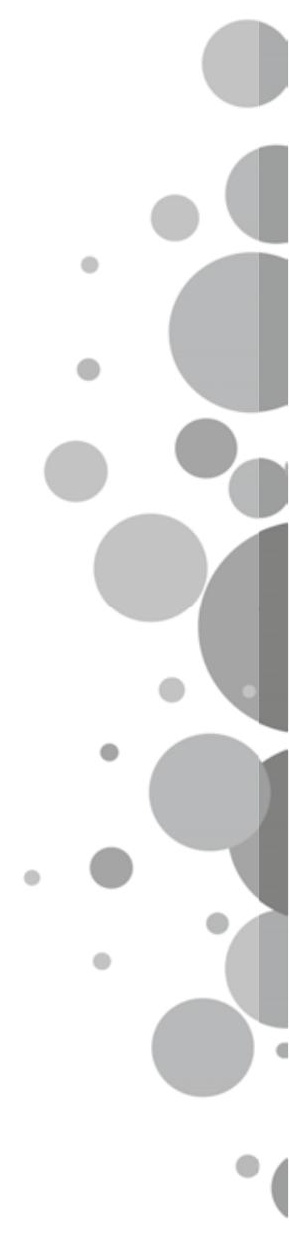




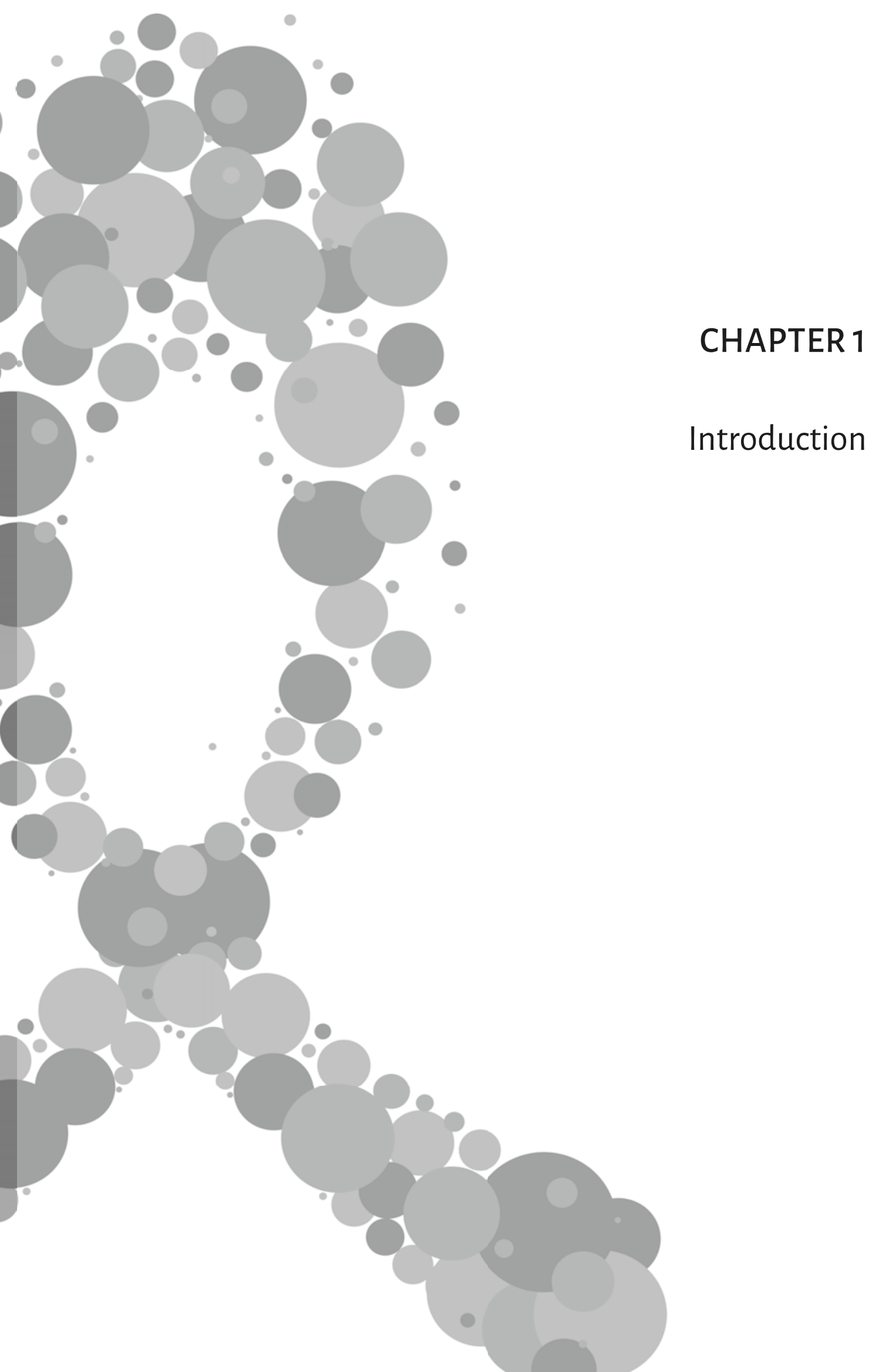





\section{Cervical Intraepithelial Neoplasia}

Cervical Intraepithelial Neoplasia (CIN) is the term used to describe dysplastic lesions of the uterine cervix. It is the precursor of cervical cancer.[1] CIN is caused by infection with human papillomavirus (HPV).[2] CIN lesions originate at the squamocolumnar junction (SC]) of the uterine cervix, which is the border between two types of epithelium covering the cervix: the glandular columnar epithelium of the endocervix and the squamous epithelium of the ectocervix. [3] Recent evidence has shown that a particular population of cells in the SC] may be the origin of $\mathrm{CIN}$ lesions: these are single layered, cuboidal epithelial cells of embryonic origin.[4] CIN lesions are classified as low-grade (CIN1) or high-grade (CIN2-3), based on the degree and extension of histopathological abnormalities. Low grade $\mathrm{CIN}$ is characterized by minor cellular atypia confined to the lower $1 / 3$ of the epithelial thickness. In high-grade $\mathrm{CIN}$, histopathological abnormalities are more pronounced and extensive: increased numbers of mitotic figures, actively replicating cells and an increased nucleus to cytoplasm ratio is seen in the lower $2 / 3$ of the epithelium (CIN2) or the complete thickness of the epithelium (CIN3).

\section{Epidemiology of CIN}

Genital infection with HPV is very common: it likely affects nearly all sexually active individuals. [5] However, most infections are successfully cleared by the immune system, leaving a small subset of approximately $10-20 \%$ of infected women with a persistent HPV infection, susceptible to development of CIN lesions.[5] In the western world, the incidence of high-grade CIN is estimated at 1.5/1000 women, with a peak incidence between the ages of 25-30 years (8.1/1000). [6] In the Netherlands, approximately 5000 women are treated for high-grade CIN lesions every year. [7] Most CIN lesions are asymptomatic and are diagnosed as a result of population screening for cervical cancer. This population screening program enables early treatment of cervical precancerous lesions and as a result, the incidence of cervical cancer is much lower than $\mathrm{CIN}$ : 6/100 o00 women in the Netherlands.[7]

\section{Human papillomavirus and oncogenesis}

CIN is caused by cervical infection with HPV, which is sexually transmitted.[2] More than 150 different HPV types (genotypes) have been identified, out of which approximately one-third infect the human genital tract. HPV genotypes are classified into risk categories according to their oncogenic potential. Twelve HPV genotypes have been classified as 'high-risk' and are associated with the development of high-grade CIN and cervical carcinoma. Among these, HPV16 and HPV18 are the most common.[3] HPV contains eight genes: six early genes (E1, E2, E4, E5, E6 and E7) regulate viral replication and DNA transcription. Of these, E6 and E7 are considered oncogenic. Two late genes (L1 and L2) encode for the virus capside proteins. Cervical HPV infection occurs at the basal cell layers of the SC]. After entry of the virus in the host cell, the viral oncogenes E6 and $\mathrm{E} 7$ are expressed and induce viral replication through activation of the host DNA replication machinery. The E7 protein binds to the retinoblastoma tumor suppressor protein (pRB) and inhibits binding of $\mathrm{pRB}$ to E2F, which leads to S-phase entry of the infected cell, inducing DNA 
replication. The E6 protein binds and degrades the host tumor suppressor protein p53, causing diminished cell-cycle blockade and apoptosis. [3, 8] These processes have been depicted in figure 1.

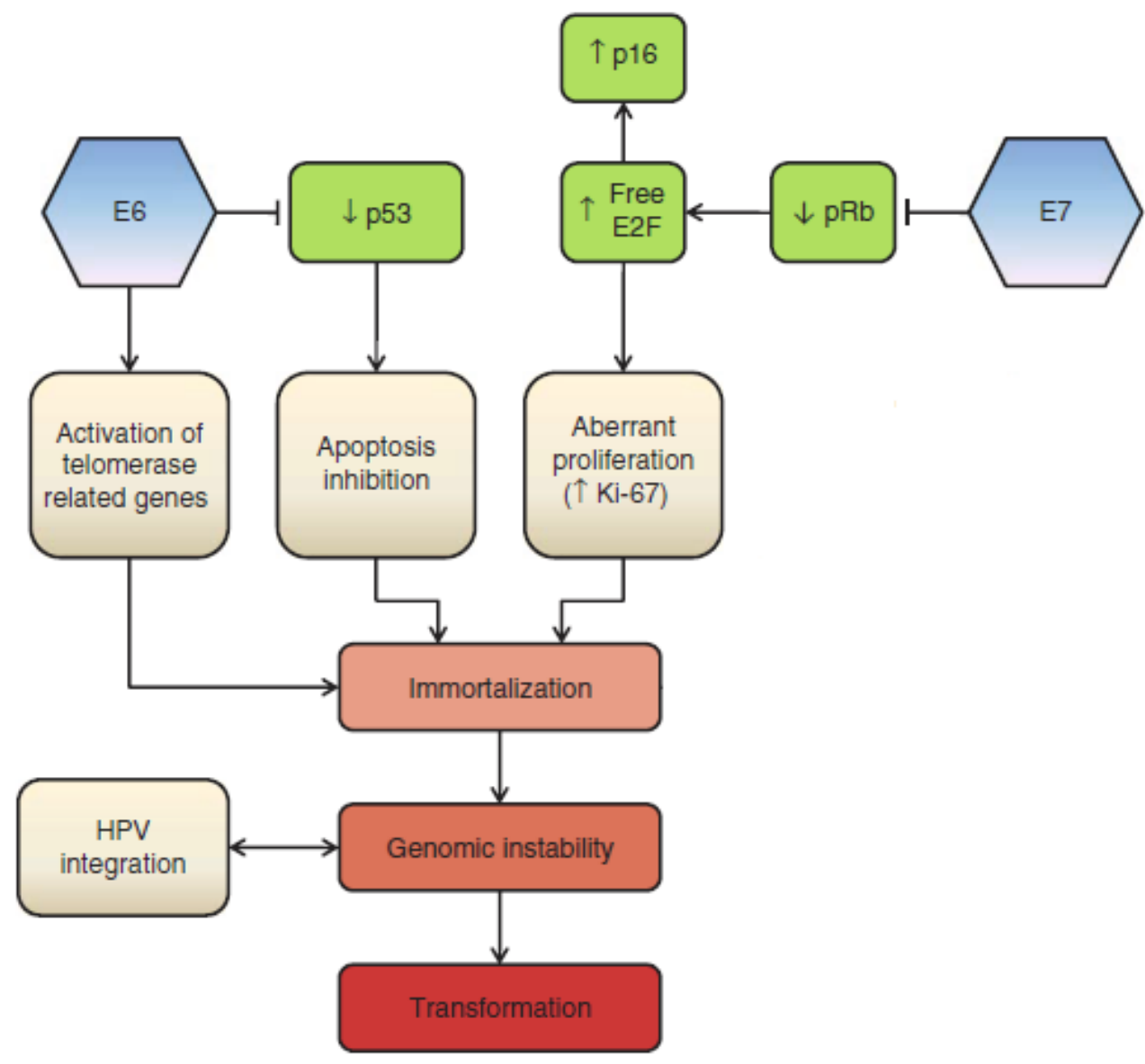

Figure 1. Cellular processes in cervical carcinogenesis following HPV infection.

E6 acts through inhibition of p53, a well-known inductor of apoptosis. Additionally, E6 leads to the increase of telomerase activity by activation of transcription of telomerase reverse transcriptase, leading to sustained telomerase, necessary for cell immortalization. E7 inactivates tumour suppressor protein retinoblastoma $(R b)$, thereby increasing free $\mathrm{E} 2 \mathrm{~F}$, leading to cellular proliferation. Cells in which the normal actions of $R b$ are suppressed by $E 7$ are characterized by an increased expression of proliferation markers, such as Ki67, and increased expression of p16. P16 is a protein that promotes cell cycle arrest through prevention of hyperphosphorylation of Rb. However, its function is bypassed by HPV, because binding of $E 7$ to $p R b$ creates a phosphorylation independent release of E2F. The combined action of E6 and E7 proteins stimulate S-phase cell cycle entry and surpassing of cell cycle checkpoints, leading to cell proliferation and immortalization. With additional accumulation of genomic instability, this forms the basis for cervical cancer development. 
Two types of infection can be distinguished. In productive infections, the expression of viral E6 and E7 is limited, leading to viral replication in normally differentiating cells of the cervical epithelium and shedding of viral particles from the superficial layers. Productive infections may lead to minor cellular reactions, which are histologically diagnosed as $\mathrm{CIN}_{1}$, or $\mathrm{CIN} 2$ at the most. Transforming infections, on the other hand, are characterized by a deregulation of viral gene expression in the basal cell layers, leading to a more abundant expression of the viral oncogenes $\mathrm{E} 6$ and E7. $[3,8]$ To date, it remains unclear whether a transforming infection is the result of a persisting and deregulated productive infection, or originates as a primary transforming infection.[9] In transforming infections, the expression of E6 and E7 induces uncontrolled cell proliferation and immortalization and makes the infected host cell susceptible to chromosomal instability. Viral integration in the host DNA and epigenetic changes further facilitate oncogenesis, by enhancing the expression of viral oncogenes and silencing of tumor-suppressor genes. Transforming infections are usually caused by high-risk HPV types and are histologically characterized by increased numbers of mitotic figures, actively replicating cells throughout the thickness of the epithelium and an increased nucleus to cytoplasm ratio. These cellular abnormalities are histologically diagnosed as high-grade $\mathrm{CIN}$ (CIN2-3). Persistent transforming infections can ultimately lead to cervical carcinoma, over a time frame of several years. [3, 8]

\section{Natural history of CIN lesions}

Not all high-grade CIN lesions would progress to cervical cancer if left untreated. It is estimated that approximately $30 \%$ of high-grade CIN lesions progress to cervical cancer, whereas spontaneous regression occurs in $20-40 \%$ of cases. $[1,10-13]$ Spontaneous regression of exclusively $\mathrm{CIN} 2$ lesions is even more common: regression rates of up to $74 \%$ have been described.[13-16] Regression of CIN lesions is induced by the host cellular immune system.[17, 18] Presentation of HPV antigens by antigen presenting cells triggers a T-cell response, comprising of cytotoxic $T$ cells (CTLs), $T$ helper cells (Th cells) and regulatory T cells (Tregs). An adequate host immune response leads to influx of CTLs, Th cells and macrophages into the HPV induced lesion, by which clearance is effected. However, in a subset of women, the immune response is insufficient for lesion clearance. This is in part due to the individual immune capacity, determined by genetic factors, acquired immunodeficiency and environmental factors such as smoking. Additionally, an insufficient immune response may be the result of immune evasion strategies of HPV and active downregulation of the immune response by the virus. Immune evasion results from the fact that HPV infection is a largely intraepithelial process with limited viremia to activate the immune system. Additionally, HPV replication does not lead to cytolysis and associated local inflammatory reactions. Active downregulation of the immune system is caused by impairment of APC functioning, inhibition of interferon synthesis and signaling, altered cytokine responses, downregulation of HLA expression on the cell surface and increased infiltration of Tregs. As such, the natural prognosis of CIN lesions is determined by a complex interaction between viral and host factors, with effects on a cellular and molecular level. 


\section{Prevention of cervical cancer}

Prevention of cervical carcinoma consists of two strategies: primary and secondary prevention. Primary prevention can be performed by HPV vaccination, but also by condom use to prevent HPV transmission. Secondary prevention can be performed by population screening for cervical cancer precursor lesions, followed by treatment.

Primary prevention of cervical carcinoma by HPV vaccination has been implemented in many countries worldwide. Young girls (and in some countries boys as well) are provided with either the bivalent vaccine, targeting HPV types 16 and 18, or the quadrivalent vaccine, which also protects against HPV types 6 and 11. Evidence has shown that vaccination of hrHPV negative women aged 15-26 years almost diminishes the incidence of HPV16/18 associated high-grade $\mathrm{CIN}$ lesions. The overall reduction of high-grade $\mathrm{CIN}$, including lesions caused by other hrHPV types, is less substantial: the incidence of any high-grade CIN lesion in these women is reduced from 287 to 106/10.000 (RR 0.37).[19] Although implementation of HPV vaccination has indeed led to a significant reduction in the prevalence of HPV 16/18 in clinical practice, the effect is restrained by a limited vaccination coverage.[20] Vaccination uptake is hampered by perceived - but invalid - safety concerns, disbelief in vaccination efficacy, parental fear that vaccination might lead to more and/or riskier sexual behavior, but also by lack of insurance coverage.[21, 22] Worldwide, the vaccination rate ranges from $39-88 \%$ of the targeted population.[23] In the Netherlands, the uptake was only 61\% in 2016.[24] It has been estimated that the coverage rate needs to be over $70 \%$ for girls aged 10-13 years, in order to substantially reduce the incidence of cervical cancer.[25] Consequently, the current vaccination program as a primary prevention strategy will not prevent all cases of HPV 16/18 induced CIN and cervical carcinoma. Moreover, the currently used bivalent and quadrivalent vaccines do not primarily protect against infection with other high-risk HPV types. A nonavalent vaccine, providing protection against HPV types $6 / 11 / 16 / 18 / 31 / 33 / 45 / 52 / 58$, has recently been approved and is now commercially available. Studies indicate an increased prevention of high-grade $\mathrm{CIN}$ with the use of this vaccine, compared to the bivalent or quadrivalent alternatives. The proportion of additional cases potentially prevented by the nonavalent vaccine is estimated to be $19-33 \%$, increasing protection against high-grade $\mathrm{CIN}$ up to $90 \%$.[26, 27] However, this nonavalent vaccine is not currently used in vaccination programs and, like the other vaccines, does not provide complete protection against HPV induced cervical lesions. Therefore, additional strategies to prevent cervical carcinoma remain necessary. Condom use provides such a strategy, by prevention of HPV transmission during sexual intercourse. However, even consistent condom use does not provide optimal protection.[28] Consequently, not all cases of cervical carcinoma will be prevented by the current primary prevention options. Therefore, secondary prevention strategies remain necessary.

Secondary prevention can be performed by population screening for cervical abnormalities, followed by treatment of cervical cancer precursor lesions. Historically, cervical cancer screening was performed by cytological assessment of a cervical smear, followed by colposcopic evaluation when cytology revealed atypical cells. However, evidence has shown that primary HPV testing, followed by cytological evaluation upon the finding of a hrHPV infection, is a more effective 
screening method. It leads to an earlier detection of persistent high-grade lesions, a $60-70 \%$ decrease in cervical carcinoma and requires less screening tests than primary cytological screening.[29-33] For this reason, many countries have changed or are changing their cervical screening program to an (at least partial) HPV-based screening method. Among these are the Netherlands, the UK, the USA, Australia, New-Zealand, Norway, Denmark, Finland, Italy, Sweden and Portugal. In this HPV based screening method, cervical cytology is first tested for the presence of hrHPV, after which cytological assessment is only done in hrHPV positive cases. Colposcopy is performed upon the finding of atypical cells. During colposcopy, either diagnostic biopsies or therapeutic excision is performed on lesions suspect for dysplasia. A definitive diagnosis (i.e. extent of dysplasia) is established based on histological examination.

\section{Current treatment of high-grade $\mathrm{CIN}$ and its pitfalls}

Until recently, guidelines advised to treat all high-grade CIN lesions, with the aim to prevent cervical cancer. The standardized treatment modality is surgical excision, which is usually performed by Large Loop Excision of the Transformation Zone (LLETZ). Surgical treatment of CIN lesions is associated with short-term side effects such as pain, prolonged bleeding and vaginal discharge, but also with the more serious long-term risk of premature birth in subsequent pregnancies.[34, 35] A recent meta-analysis shows that treatment of CIN with LLETZ increases the risk of overall premature birth ( $<37$ weeks, RR 1.58 [1.37-1.81]), but also the risk of severe $(<32-$ 34 weeks) and extreme premature (<28-30 weeks) birth (RR 2.13 [1.66-2.75] and 2.57 [1.97-3.35] respectively).[35] Interestingly, women with CIN showed a baseline increased risk of prematurity, regardless of treatment procedure. Nevertheless, increasing cone depth and repeated treatment further increased this risk, indicating that the increased risk of premature birth is at least in part due to cervical insufficiency after partial cervical excision. As CIN commonly affects young women in their fertile ages, this is a relevant side effect and prevention of unnecessary surgical treatment should be pursued in women with a future pregnancy desire. For this reason, guidelines have recently introduced the option of observational management for $\mathrm{CIN} 2$ lesions for younger women, as these lesions often show spontaneous regression and progression of $\mathrm{CIN} 2$ to cervical carcinoma on short term is rare.[36] Follow-up is performed by cytological examination. The recommendation upon conservative management of CIN2 lesions has led to a decrease in LLETZ treatment of $\mathrm{CIN} 2$ lesions, as was shown in a study among Boston women: LLETZ treatment of $\mathrm{CIN} 2$ decreased from $55 \%$ to $18 \%(p=0.04)$ in women aged $18-23$. For women over 24 , however, the LLETZ rate remained stable (70\% vs $74 \%, p=0.72)$.[37] Although the treatment rate for $\mathrm{CIN}_{2}$ has declined, not all (young) women with a $\mathrm{CIN} 2$ lesion receive conservative management, for reasons unknown. Many of these women may become pregnant in the future and are thus at risk for premature birth. $\mathrm{CIN} 3$ lesions remain to be primarily treated by LLETZ, as they carry a higher risk of progression to cervical carcinoma than $\mathrm{CIN} 2$ and their potential of spontaneous regression is lower. These women too, are at risk for side effects. Importantly, a subset of their lesions would show spontaneous regression if left untreated, so overtreatment also occurs in women with CIN3. Taken together, a significant number of women with high-grade $\mathrm{CIN}$ is at risk for complications as a result of surgical treatment, including a subset of women whose lesions would show spontaneous regression if left untreated. To reduce the surgical treatment rate of high-grade CIN lesions, with the aim 
to reduce side effects, strategies are needed towards conservative management of high-grade CIN, without increasing the risk of lesion progression to cervical cancer.

\section{Strategies towards observational and non-surgical management of high-grade CIN}

Two strategies can be identified with the aim to reduce surgical (over)treatment of high-grade CIN. These include (1) observational management of CIN lesions that are likely to show spontaneous regression and (2) non-surgical treatment modalities for CIN lesions.

\section{Observational management of $\mathrm{CIN}$}

A first strategy is observational management of CIN lesions that are likely to show spontaneous regression. As discussed before, (inter)national guidelines now advice upon observational managementofCIN2 lesions inyoungerwomen.[36] However, for reasons unknown, observational management is not applied to all young women, leaving a subset at risk for side effects of potentially unnecessary - surgical treatment.[37] Additionally, a subset of women with a $\mathrm{CIN} 3$ lesion undergoes unnecessary treatment, as their lesions would show spontaneous regression if left untreated. Ideally, the individual disease outcome would be predictable, in order to select those women with $\mathrm{CIN} 2$ lesions and $\mathrm{CIN} 3$ lesions in which spontaneous regression is expected. Current histopathological examination cannot distinguish those lesions that will regress from those that will not. Therefore, other markers are needed: individual or combined markers that predict the natural course of individual CIN lesions. Such prognostic biomarkers are clinical or biological characteristics that are objectively measurable and provide information on the likely outcome of disease in an individual.[38] In case of CIN lesions, prognostic biomarkers should be identified at the different levels of interaction between viral and host factors in their natural history. Potential prognostic biomarkers may be identified at the level of general patient and lesion characteristics (such as smoking status, HPV genotype and lesion size) or on the level of functional cellular mechanisms in the pathogenesis and regression of CIN lesions (such as proliferation markers, immune markers or genetic markers). Clinically suitable biomarkers should meet several criteria, regarding assessment, test characteristics and decision-making. There should be an accurate and reproducible analytical method and the assay should be readily available at reasonable cost. There must be a strong and consistent association between the biomarker and disease outcome, preferably verified in multiple studies. Finally, decision-limits should be available and validated. [39] A variety of prognostic molecular markers in the natural history of both low-and high-grade $\mathrm{CIN}$ lesions has been reported in the literature. An overview of these markers and critical appraisal of their clinical applicability, however, is lacking. Determination of the clinical applicability of the identified markers can be performed according to the prospective-specimen-collection, retrospective-blinded-evaluation (PROBE) design. This design advises upon the development of a functional biomarker and consists of four key components: clinical context and outcome, biomarker performance criteria, biomarker test characteristics and study size. [40] An overview of the natural history of high-grade CIN and critical appraisal of previously studied prognostic molecular markers according to the PROBE criteria could clarify their clinical applicability. Furthermore, it could identify molecular markers that deserve further evaluation as prognostic biomarkers in high-grade CIN. 
While counseling of patients for conservative management of $\mathrm{CIN} 3$ cannot yet be adequately applied, this is already a matter of common practice for CIN2. Nevertheless, not all young women with $\mathrm{CIN} 2$ receive conservative treatment. The reasons for this are unknown, but may be found in patient factors (fear of progression, desire for a fast solution) or physician factors (fear of progression, performing surgical treatment by habit). A better individual prediction of disease outcome would enable a more adequate patient counseling with regard to management options. As conservative treatment of $\mathrm{CIN} 2$ is already implemented in clinical practice, it is undesirable to use complex and expensive biomarkers to predict disease outcome. Rather, simple clinical parameters - based on patient and lesion characteristics - may be applied to predict individual disease outcome and thus enable a more personalized counseling with regard to management options. Since the implementation of HPV-based screening for cervical cancer, nearly all newly diagnosed $\mathrm{CIN}_{2}$ lesions are now hrHPV positive. Identification of prognostic factors for disease outcome in exclusively hrHPV positive CIN2 is therefore vital, in order to provide with clinically applicable prognostic factors.

Taken together, the identification of prognostic markers in high-grade CIN may enable individual prediction of disease outcome, in order to select those women with a high likelihood of spontaneous regression for conservative management.

\section{Non-surgical treatment of CIN}

Another strategy to reduce side effects of surgical treatment of CIN lesions is the application of non-surgical treatment modalities. Non-surgical ablative methods (electrocautery, cryotherapy and laser ablation) have been proven effective and were widely used until the 1990s. After the introduction of LLETZ treatment in the 1990s, several first studies indicated a potential undertreatment of invasive cancer by ablative methods, for which reason these methods were abandoned and replaced by surgical excision.[41] The fact that meta-analytic data shows that cryotherapy and laser ablation have comparable efficacy to excisional techniques has not lead to their re-introduction.[41, 42] More recently, several other non-surgical approaches have been evaluated in the treatment of CIN.[43] Among these are photodynamic therapy and 5-fluorouracil (5-FU), which are applied as direct cervical irritants. Both have not reached clinical implementation, due to limited evidence but also high costs and toxicity. Other research is focusing on immunotherapy for $\mathrm{CIN}$, with the aim to enhance the immune response to $\mathrm{CIN}$ lesions in order to promote disease regression. Several forms of immunotherapy have been studied: local and systemic treatment with antiviral drugs, local treatment with interferon and imiquimod and therapeutic HPV vaccination. Regarding antiviral drugs, local application of cidofovir has been shown to be moderately effective in small trials. It is not likely to be clinically applied due to potential mutagenic and carcinogenic effects and high costs. Local and systemic application of HIV protease inhibitors has shown promising results in vitro and in small trials, but has not yet been studied beyond a phase II trial. Intralesional injection of interferon has been proven very effective in CIN lesions in one study, but has not been investigated further. This may be due to high costs and significant side effects. HPV vaccination is now extensively studied as systemic immunotherapy in CIN.[44-46] The currently available prophylactic HPV vaccines have not been successful in treating established HPV infections. These prophylactic vaccines prevent HPV infection by targeting the humoral immune response through delivering virus- 
like particles (VLPs), encoding late HPV viral capsid proteins L1 and/or L2. T-helper cells activate B-cells to become plasma cells, which results in the generation of antibodies. These antibodies block HPV infection and induce protection from HPV. In contrast, therapeutic HPV vaccines must activate cell-mediated immunity. Antigen-presenting cells (APCs) induce priming of T-cells into effector cells. CD8+ T cells, or cytotoxic T lymphocytes (CTLs), mediate the antigen-specific killing of tumor cells and are, as such, responsible for clearance of CIN lesions. The deletion of L1 and L2 genes during HPV integration and deregulation of $\mathrm{E} 6$ and $\mathrm{E} 7$ expression in high-grade $\mathrm{CIN}$ renders the currently available prophylactic vaccines ineffective in targeting infected cells. $[45,46]$ Vaccines targeting other viral immunogens, mainly E6 and E7 oncogenes, have been developed and tested, but the development of an effective and clinically applicable therapeutic vaccine has proven challenging. The greatest challenge is the relative inaccessibility of HPV due to previously described immune evasion strategies of the virus. [44] Several different categories of vaccines have been produced and tested, which differ in the type of immunogen and delivery system. These different categories include vaccines based on bacterial vector vaccines, viral vector, peptides, proteins, DNA, RNA, dendritic cells and tumor cells. [46] DNA vaccines have emerged as the most promising form of therapeutic HPV vaccines, due to their safety, stability, manufacturability and ability to induce antigen-specific immunity.[45] However, clinical efficacy remains limited. Several phase I/II studies have shown efficacy rates of 30\% to $78 \%$.[45] One trial included a placebo group. Regression occurred in $48 \%$ of vaccinated women with $\mathrm{CIN} 2 / 3$, compared to $30 \%$ in the placebo group ( $p=0.034)$. [47] Additional trials are ongoing, which also focus on enhancement of the vaccination effect by co-treatment with adjuvants. [44-46].

Currently, the only form of immunotherapy that is incorporated in the Dutch guideline for treatment of CIN is imiquimod cream.[7] Imiquimod enhances anti-viral and anti-tumor immunity and has been registered for treatment of genital warts and basal cell carcinoma. In the gynecological discipline, it is used to treat genital warts as well as vulvar intraepithelial neoplasia (VIN), both HPV-related diseases alike CIN. In VIN, treatment efficacy rates up to $88 \%$ have been reported.[48] Following the application in VIN, imiquimod was also studied in patients with high-grade CIN lesions. The only RCT was performed by Crimm et al., who included 59 women with high-grade CIN.[49] Disease regression and remission was more common in the imiquimod group, compared to a placebo group (73 vs $39 \%, p<0.05$ and 47 vs $14 \%, p<0.05$ ). Based on this study, treatment of high-grade CIN with imiquimod seems feasible and could reduce side effects of surgical treatment. However, despite the fact that imiquimod is incorporated in the Dutch guideline for treatment of $\mathrm{CIN}$, its application in clinical practice seems limited. An important limiting factor may be a perceived lack of evidence regarding treatment efficacy. Indeed, evidence on efficacy is based on one relatively small study and long-term results in terms of recurrence rates are lacking. Clinical application of a new treatment modality warrants robust evidence of efficacy. For this reason, additional research on both short-and long-term efficacy of imiquimod for high-grade CIN seems necessary. Clinical application could also be limited by unawareness of physicians regarding imiquimod application in CIN, or unwillingness to apply it due to other reasons, such as side effects. An inventory of the awareness, attitudes and current clinical experience of gynecologists regarding imiquimod treatment of high-grade CIN could explain the limited clinical application by physicians and indicate what conditions are considered important for clinical application. 
A last limitation of clinical application could be unwillingness of women to receive imiquimod treatment. Indeed, although imiquimod treatment could potentially prevent side effects of surgical treatment, the treatment modality has several important downsides. First, treatment efficacy does not compare to the efficacy of LLETZ treatment. Second, imiquimod treatment is more labor intensive and time consuming for patients than surgical treatment. Last, side effects of imiquimod treatment were very common and sometimes severe: almost all women treated with imiquimod experienced at least one side effect, among which were vulvar pain or pruritus, headache, myalgia, flu-like symptoms and vulvar erythema, erosion, edema or ulceration. Severe vulvar pain or pruritus was reported by $7 \%$ of women and severe flu-like symptoms by $13 \%$. These treatment characteristics may influence the willingness of women to receive imiquimod treatment as an alternative to surgical treatment. It seems likely that imiquimod treatment will not be preferred by all women with high-grade CIN. Rather, those women with a future pregnancy desire may consider imiquimod treatment to prevent future obstetric complications. In the era of personalized medicine and shared-decision-making, patient preferences are considered increasingly important.[50] It is therefore essential to understand patient preferences with regard to the choice between imiquimod or surgery of high-grade CIN. A patient preference study could clarify which women would prefer imiquimod treatment instead of surgery, and at what terms. [51] Based on the above, additional evidence is necessary to assess the clinical applicability of imiquimod as a treatment modality for high-grade $\mathrm{CIN}$, in the fields of treatment efficacy, physician awareness and attitudes, and patient preferences.

\section{Aims of the current thesis}

The aims of the studies described in this thesis are:

1. Assessment of clinical and molecular biomarkers as predictors of spontaneous regression of high-grade CIN lesions.

For this purpose, we first summarized the complex interaction between the immune system, viral factors and functional cellular mechanisms that determine the natural history of CIN lesions and conducted a review on prognostic biomarkers in high-grade CIN. This review is presented in chapter 2. Several biomarkers were identified that are associated with the natural history of highgrade $\mathrm{CIN}$ or can be regarded as promising in this regard. We further evaluated the prognostic properties of two of these biomarkers (HLA alleles and the chromosomal 3926 locus). Chapter 3 describes the prognostic properties of HLA alleles in the natural history of high-grade CIN. Chapter 4 describes the prognostic properties of the chromosomal 3926 locus in the natural history of high-grade CIN. Additionally, we aimed to identify biomarkers for the prediction of disease outcome in $\mathrm{CIN} 2$, to improve counseling of these patients for conservative management. Chapter 5 describes the development a prediction model for disease outcome of CIN 2. This prediction model is based on simple clinical parameters, reflecting patient and lesion characteristics, in order to provide with a widely applicable prediction model. After the development of this model, the hrHPV based screening method was implemented. As a consequence, nearly all CIN2 lesions are now hrHPV positive. We therefore aimed to identify prognostic factors in this subgroup of lesions as well, as described in chapter 6. 
2. Assessment of clinical applicability of imiquimod cream as a treatment modality for highgrade CIN.

For this purpose, we assessed the knowledge, experiences and attitudes of physicians regarding imiquimod treatment of high-grade $\mathrm{CIN}$ and the preferences of $\mathrm{CIN}$ patients for either surgical treatment or imiquimod treatment. The results of these studies are presented in chapter 7 and 8. Chapter 9 contains the study protocol of the TOPical Imiquimod treatment of high-grade CIN (TOPIC) trial. This randomized controlled trial was designed to assess short and long-term efficacy of imiquimod treatment in high-grade CIN, as well as side effects and quality of life associated with the treatment modality, comparing imiquimod treatment to conventional treatment by LLETZ. Due to lagging inclusions, the study was prematurely terminated and converted to a nonrandomized trial, in which patients could choose the treatment modality of their preference. Details as to the termination of the study are described in the same chapter. The adjusted, nonrandomized, study (TOPIC-3) is currently running in three centers in the Netherlands. All 120 women have been included at the time of writing. The first study results are expected in the spring of 2019.

Taken together, the studies in this thesis aim to provide a basis for alternative strategies in the treatment of high-grade $\mathrm{CIN}$, with a focus on observational management of women with a high probability of disease regression and non-surgical treatment with imiquimod for women who prefer this above surgical treatment. 


\section{References}

1. McCredie MR, Sharples K], Paul C, Baranyai ], Medley G, Jones RW, et al. Natural history of cervical neoplasia and risk of invasive cancer in women with cervical intraepithelial neoplasia 3: a retrospective cohort study. Lancet Oncol. 2008;9(5):425-34.

2. zur Hausen $\mathrm{H}$. Papillomaviruses causing cancer: evasion from host-cell control in early events in carcinogenesis. J Natl Cancer Inst. 2000;92(9):690-8.

3. Doorbar ], Quint W, Banks L, Bravo IC, Stoler M, Broker TR, et al. The biology and life-cycle of human papillomaviruses. Vaccine. 2012;30 Suppl 5:F55-70.

4. Herfs M, Yamamoto Y, Laury A, Wang X, Nucci MR, McLaughlin-Drubin ME, et al. A discrete population of squamocolumnar junction cells implicated in the pathogenesis of cervical cancer. Proc Natl Acad Sci U S A. 2012;109(26):10516-21.

5. Trottier H, Franco EL. The epidemiology of genital human papillomavirus infection. Vaccine. 2006;24 Suppl 1:S1-15.

6. Insinga RP, Glass AG, Rush BB. Diagnoses and outcomes in cervical cancer screening: a population-based study. Am J Obstet Gynecol. 2004;191(1):105-13.

7. Landelijke richtlijn CIN, AIS en VAIN. Versie 1.0, 2015. Integraal Kankercentrum Nederland. https://www.nhg.org/sites/default/files/content/nhg_org/uploads/cin_ais_en_vain.pdf Accessed at july 9, 2018. .

8. Moody CA, Laimins LA. Human papillomavirus oncoproteins: pathways to transformation. Nat Rev Cancer. 2010;10(8):550-60.

9. Steenbergen RD, Snijders P], Heideman DA, Meijer C]. Clinical implications of (epi)genetic changes in HPV-induced cervical precancerous lesions. Nat Rev Cancer. 2014;14(6):395-405.

10. Ostor AC. Natural history of cervical intraepithelial neoplasia: a critical review. Int ] Gynecol Pathol. 1993;12(2):186-92.

11. Munk AC, Gudlaugsson E, Ovestad IT, Lovslett K, Fiane B, Hidle B, et al. Interaction of epithelial biomarkers, local immune response and condom use in cervical intraepithelial neoplasia 2-3 regression. Gynecol Oncol. 2012;127(3):489-94.

12. Trimble CL, Piantadosi S, Gravitt P, Ronnett B, Pizer E, Elko A, et al. Spontaneous regression of high-grade cervical dysplasia: effects of human papillomavirus type and HLA phenotype. Clin Cancer Res. 2005;11(13):4717-23.

13. Lee MH, Finlayson S], Gukova K, Hanley G, Miller D, Sadownik LA. Outcomes of Conservative Management of High Grade Squamous Intraepithelial Lesions in Young Women.] Low Cenit Tract Dis. 2018;22(3):212-8.

14. Holowaty P, Miller AB, Rohan T, To T. Natural history of dysplasia of the uterine cervix.] Natl Cancer Inst. 1999;91(3):252-8.

15. Discacciati MG, de Souza CA, d'Otavianno MG, Angelo-Andrade LA, Westin MC, RabeloSantos $\mathrm{SH}$, et al. Outcome of expectant management of cervical intraepithelial neoplasia grade 2 in women followed for 12 months. Eur ] Obstet Cynecol Reprod Biol. 2011;155(2):2048.

16. Tainio K, Athanasiou A, Tikkinen KAO, Aaltonen R, Cardenas ], Hernandes, et al. Clinical course of untreated cervical intraepithelial neoplasia grade 2 under active surveillance: systematic review and meta-analysis. BM]. 2018;360:k499. 
17. Stanley MA. Epithelial cell responses to infection with human papillomavirus. Clin Microbiol Rev. 2012;25(2):215-22.

18. Patel S, Chiplunkar S. Host immune responses to cervical cancer. Curr Opin Obstet Cynecol. 2009;21(1):54-9.

19. Arbyn M, Xu L, Simoens C, Martin-Hirsch PP. Prophylactic vaccination against human papillomaviruses to prevent cervical cancer and its precursors. Cochrane Database Syst Rev. 2018;5:CDoo9069.

20. Mesher D, Panwar K, Thomas SL, Edmundson C, Choi YH, Beddows S, et al. The Impact of the National HPV Vaccination Program in England Using the Bivalent HPV Vaccine: Surveillance of Type-Specific HPV in Young Females, 2010-2016. J Infect Dis. 2018.

21. Newman PA, Logie CH, Lacombe-Duncan A, Baiden P, Tepjan S, Rubincam C, et al. Parents' uptake of human papillomavirus vaccines for their children: a systematic review and metaanalysis of observational studies. BM] Open. 2018;8(4):e019206.

22. Donken R, Tami A, Knol M], Lubbers K, van der Sande MAB, Nijman HW, et al. Changes in (risk) behavior and HPV knowledge among Dutch girls eligible for HPV vaccination: an observational cohort study. BMC Public Health. 2018;18(1):837.

23. Bruni L, Diaz M, Barrionuevo-Rosas L, Herrero R, Bray F, Bosch FX, et al. Clobal estimates of human papillomavirus vaccination coverage by region and income level: a pooled analysis. Lancet Clob Health. 2016;4(7):e453-63.

24. Vaccinatiegraad Rijksvaccinatieprogramma Nederland. Jaarverslag 2016. RIVM Rapport 2016-0064. Accessible at: https://www.rivm.nl/dsresource?objectid=89ece336-f681-43c2abco-7213e87f6b6b\&type=org\&disposition=inline. Accessed Juli 9, 2018.

25. Human papillomavirus vaccines. WHO position paper. Wkly Epidemiol Rec. 2009 Apr 10;84(15):118-31.

26. Capra G, Giovannelli L, Matranga D, Bellavia C, Guarneri MF, Fasciana T, et al. Potential impact of a nonavalent HPV vaccine on HPV related low-and high-grade cervical intraepithelial lesions: A referral hospital-based study in Sicily. Hum Vaccin Immunother. 2017;13(8):183943.

27. Bogani C, Taverna F, Lombardo C, Signorelli M, Chiappa V, Casarin ], et al. Potential impact of introducing a nonavalent HPV vaccination. Int ] Cynaecol Obstet. 2018.

28. Lam JU, Rebolj M, Dugue PA, Bonde ], von Euler-Chelpin M, Lynge E. Condom use in prevention of Human Papillomavirus infections and cervical neoplasia: systematic review of longitudinal studies. J Med Screen. 2014;21(1):38-50.

29. Ronco G, Giorgi-Rossi P, Carozzi F, Confortini M, Dalla Palma P, Del Mistro A, et al. Efficacy of human papillomavirus testing for the detection of invasive cervical cancers and cervical intraepithelial neoplasia: a randomised controlled trial. Lancet Oncol. 2010;11(3):249-57.

30. Rijkaart DC, Berkhof ], Rozendaal L, van Kemenade F], Bulkmans NW, Heideman DA, et al. Human papillomavirus testing for the detection of high-grade cervical intraepithelial neoplasia and cancer: final results of the POBASCAM randomised controlled trial. Lancet Oncol. 2012;13(1):78-88.

31. Wright TC, Stoler MH, Behrens CM, Sharma A, Zhang G, Wright TL. Primary cervical cancer screening with human papillomavirus: end of study results from the ATHENA study using HPV as the first-line screening test. Gynecol Oncol. 2015;136(2):189-97. 
32. Koliopoulos G, Nyaga VN, Santesso N, Bryant A, Martin-Hirsch PP, Mustafa RA, et al. Cytology versus HPV testing for cervical cancer screening in the general population. Cochrane Database Syst Rev. 2017;8:CDo08587.

33. Ronco G, Dillner ], Elfstrom KM, Tunesi S, Snijders P], Arbyn M, et al. Efficacy of HPV-based screening for prevention of invasive cervical cancer: follow-up of four European randomised controlled trials. Lancet. 2014;383(9916):524-32.

34. Group T, Sharp L, Cotton S, Cochran C, Gray N, Little ], et al. After-effects reported by women following colposcopy, cervical biopsies and LLETZ: results from the TOMBOLA trial. BJOC. 2009;116(11):1506-14.

35. Kyrgiou M, Athanasiou A, Kalliala IE], Paraskevaidi M, Mitra A, Martin-Hirsch PP, et al. Obstetric outcomes after conservative treatment for cervical intraepithelial lesions and early invasive disease. Cochrane Database Syst Rev. 2017;11:CD012847.

36. Massad LS, Einstein MH, Huh WK, Katki HA, Kinney WK, Schiffman M, et al. 2012 updated consensus guidelines for the management of abnormal cervical cancer screening tests and cancer precursors. J Low Genit Tract Dis. 2013;17(5 Suppl 1):S1-S27.

37. Perkins RB, Jorgensen JR, McCoy ME, Bak SM, Battaglia TA, Freund KM. Adherence to conservative management recommendations for abnormal pap test results in adolescents. Obstet Gynecol. 2012;119(6):1157-63.

38. Italiano A. Prognostic or predictive? It's time to get back to definitions! ] Clin Oncol. 2011;29(35):4718; author reply -9.

39. Morrow DA, de Lemos]A. Benchmarks for the assessment of novel cardiovascular biomarkers. Circulation. 2007;115(8):949-52.

40. Pepe MS, Feng Z, Janes H, Bossuyt PM, Potter JD. Pivotal evaluation of the accuracy of a biomarker used for classification or prediction: standards for study design. ] Natl Cancer Inst. 2008;100(20):1432-8.

41. Khan M], Smith-McCune KK. Treatment of cervical precancers: back to basics. Obstet Gynecol. 2014;123(6):1339-43.

42. Kyrgiou M, Tsoumpou I, Vrekoussis T, Martin-Hirsch P, Arbyn M, Prendiville W, et al. The upto-date evidence on colposcopy practice and treatment of cervical intraepithelial neoplasia: the Cochrane colposcopy \& cervical cytopathology collaborative group (C5 group) approach. Cancer Treat Rev. 2006;32(7):516-23.

43. Hampson L, Martin-Hirsch P, Hampson IN. An overview of early investigational drugs for the treatment of human papilloma virus infection and associated dysplasia. Expert Opin Investig Drugs. 2015;24(12):1529-37.

44. Trimble CL. Towards identifying new therapeutic targets in cancers attributable to HPV. Gynecol Oncol. 2018;149(2):225-6.

45. Cheng MA, Farmer E, Huang C, Lin ], Hung CF, Wu TC. Therapeutic DNA Vaccines for Human Papillomavirus and Associated Diseases. Hum Cene Ther. 2018.

46. Yang A, Jeang ], Cheng K, Cheng T, Yang B, Wu TC, et al. Current state in the development of candidate therapeutic HPV vaccines. Expert Rev Vaccines. 2016;15(8):989-1007. 
47. Trimble CL, Morrow MP, Kraynyak KA, Shen X, Dallas M, Yan J, et al. Safety, efficacy, and immunogenicity of VGX-3100, a therapeutic synthetic DNA vaccine targeting human papillomavirus 16 and 18 E6 and E7 proteins for cervical intraepithelial neoplasia 2/3: a randomised, double-blind, placebo-controlled phase 2 b trial. Lancet. 2015;386(10008):207888.

48. de Witte C], van de Sande A], van Beekhuizen H], Koeneman MM, Kruse A], Gerestein CC. Imiquimod in cervical, vaginal and vulvar intraepithelial neoplasia: a review. Gynecol Oncol. 2015;139(2):377-84.

49. Crimm C, Polterauer S, Natter C, Rahhal ], Hefler L, Tempfer CB, et al. Treatment of cervical intraepithelial neoplasia with topical imiquimod: a randomized controlled trial. Obstet Gynecol. 2012;120(1):152-9.

50. Legare F, Witteman HO. Shared decision making: examining key elements and barriers to adoption into routine clinical practice. Health Aff (Millwood). 2013;32(2):276-84.

51. Dirksen CD. The use of research evidence on patient preferences in health care decisionmaking: issues, controversies and moving forward. Expert Rev Pharmacoecon Outcomes Res. 2014;14(6):785-94. 


\section{PART 1}

Assessment of clinical and molecular biomarkers as predictors for spontaneous regression of high-grade $\mathrm{CIN}$ lesions 


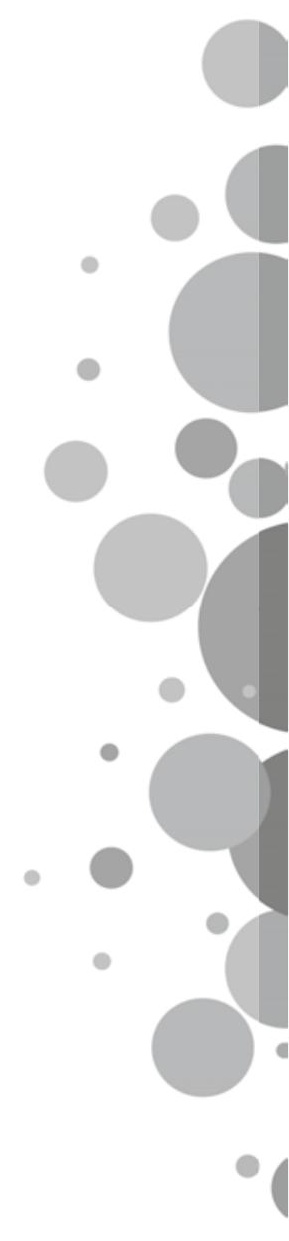




\section{CHAPTER 2}

Natural history of high-grade cervical intraepithelial neoplasia: a review of prognostic biomarkers

Margot M Koeneman, Roy FPM Kruitwagen, Hans W Nijman, Brigitte F Slangen, Toon van Corp, Arnold ] Kruse

Expert Review of Molecular Diagnostics. 2015 Apr;15(4):527-46. 


\section{Abstract}

The natural history of high-grade cervical intraepithelial neoplasia (CIN) is largely unpredictable and current histopathological examination is unable to differentiate between lesions that will regress or not. Therefore, most high-grade lesions are currently treated by surgical excision, leading to overtreatment and unnecessary complications. Prognostic biomarkers may differentiate between lesions that will regress or not, making individualized treatment of high-grade CIN possible. This review identified several promising prognostic biomarkers. These biomarkers include viral genotype and viral DNA methylation (viral factors), HLA-subtypes, markers of lymphoproliferative response, telomerase amplification and HPV-induced epigenetic effects (host factors) and Ki67, p53 and pRb (cellular factors). All identified biomarkers were evaluated according to their role in the natural history of high-grade $\mathrm{CIN}$ and according to established criteria for evaluation of biomarkers (PROBE-criteria). None of the biomarkers meet the PROBE criteria for clinical applicability. More research on prognostic biomarkers in high-grade $\mathrm{CIN}$ is necessary. 


\section{Introduction}

High-grade cervical intraepithelial neoplasia $(\mathrm{CIN})$ is considered to be the precursor of cervical cancer, which is currently the fourth most common malignancy in women worldwide.(1) Cervical cancer, and high-grade $\mathrm{CIN}$ as its precursor, are caused by infection with human papillomavirus (HPV).(2) The incidence of cervical cancer has declined in developed countries as a result of screening programs and early treatment of precursor lesions.(3) Currently, standard therapy for high-grade $\mathrm{CIN}$ lesions is surgical excision: this is usually done by large loop excision of the transformation zone (LLETZ). The rationale for this generalized treatment is that the natural history of the individual CIN lesion is unpredictable: whereas a subset of high-grade lesions will progress to cervical cancer, another subset will regress spontaneously. Approximately $30 \%$ of high-grade CIN progresses to cervical cancer. $(4,5)$ On the contrary, recent evidence suggests that spontaneous regression of high-grade $\mathrm{CIN}$ occurs in approximately $20-40 \%$ of high-grade lesions.(6-9) Current histopathological assessment is unable to differentiate between lesions that will progress to cervical cancer and those that will regress spontaneously. In the light of these findings, treatment of all high-grade CIN leads to overtreatment and unnecessary complications as a result.(10) LLETZ is an invasive procedure with several short term and long term side effects. Two important long term adverse effects of LLETZ treatment are a potentially increased risk of subfertility and increased risk of premature birth in subsequent pregnancies, most probably as a result of cervical insufficiency.(11-14) Ideally, those lesions that will regress spontaneously should be differentiated from those that will not do so, allowing for watchful waiting instead of invasive treatment in the subgroup of patients in which spontaneous regression is expected. Individual regression risk prediction of high-grade CIN is essential for this strategy. As current histopathological assessment is not suited for this purpose, other markers are needed: individual or combined markers that predict the natural course of individual high-grade CIN lesions.

Such prognostic biomarkers are clinical or biological characteristics that are objectively measurable and provide information on the likely outcome of disease outcome in an individual. (15) Prognostic biomarkers define the effects of patient or disease characteristics on disease outcome in patients. As such, prognostic biomarkers may be helpful for the identification of patients with high risk of disease progression thereby guiding the choice to select those who will benefit from treatment.

The natural history of $\mathrm{CIN}$ was historically assumed a sequence of steps through histopathological classification. More recent insights indicate that the natural history of CIN should rather reflect HPV infection status: it is hypothesized that HPV infections are either productive or transforming. Productive infections lead to the production of new viruses and are histologically characterized by minor cellular changes, diagnosed as $\mathrm{CIN} 1$ or less. On the contrary, high-grade $\mathrm{CIN}$ is caused by a transforming infection, leading to more extensive cellular changes.(16) The development of CIN is determined by a complex interaction between the immune system, viral factors and functional cellular mechanisms. Potential prognostic biomarkers may be identified at these different levels of interaction.(17-19) This review aims to identify biomarkers that have been associated with the natural history of high-grade $\mathrm{CIN}$ or are considered promising in this regard. Before biomarker 
results can be used in individualized medical decision making, it is crucial to know their accuracy in predicting the behaviour of high-grade CIN lesions. Each biomarker will be evaluated according to established criteria to determine applicability of diagnostic and prognostic candidate biomarkers: the prospective-specimen-collection, retrospective-blinded-evaluation (PROBE) design.(20) Selected biomarkers may function as prognostic biomarkers in individual high-grade $\mathrm{CIN}$ lesions. This enables a more individualized treatment plan for patients, with the option of watchful waiting in those patients in which spontaneous regression is likely.

\section{HPV: infection and oncogenesis}

Papillomaviruses are species-specific, double strand DNA viruses. To date, over 150 different types have been identified that infect human beings and of these, approximately one third infect squamous epithelia of the genital tract (http://pave.niaid.nih.gov/\#home). The virus contains eight coding genes. Six early genes (E1, E2, E4, E5, E6 and E7) regulate viral replication and DNA transcription, of which E6 and E7 are considered oncogenic. Two late genes (L1 and L2) encode for the virus capside proteins. Human papillomaviruses are categorized according to their oncogenic potential. Although the individual oncogenic potential shows a gradient pattern, all viruses are categorized into either high risk, intermediate risk or low risk types. Twelve HPV-types have been consistently classified as high-risk. (21) High-grade CIN and cervical carcinoma are predominantly associated with high-risk HPV types.(22) High risk types HPV16 and HPV-18 are the most prevalent virus types in cervical carcinoma. Either of these types are identified in approximately $70 \%$ of cervical malignancies.(23)

\section{Infection}

Infection of the anogenital tract with HPV is very common: up to $80 \%$ of the female population may be infected at least once in a lifetime. $(24,25)$ Most infections are successfully cleared, leaving a small subset of approximately $10-20 \%$ of infected women with a persistent HPV infection, susceptible to development of CIN. $(24,26,27)$. The biology and life-cycle of HPV and its role in cervical oncogenesis has been extensively reviewed by others. $(28,29)$ A short summary will be provided here, as a basis for biomarker selection

\section{Productive infection}

HPV infection results primarily in a productive infection of the cervical epithelium in which new viruses are produced. Infection occurs at the basal cell layers of the squamocolumnar junction, after which viral DNA is present in an episomal form and expression of E6 and E7 is limited. Viral replication is facilitated by the host replication apparatus and leads to abundant expression of viral genes, including E6 and E7, in differentiating cells in the upper cell layers. Viral replication occurs, capside proteins are produced and new viruses are formed, which are shed from the upper cell layers. Productive infections are cytologically and histologically characterized by koilocytosis and other minor cellular reactions, which are roughly diagnosed as CIN 1 or CIN 2 at the most (figure 1). 

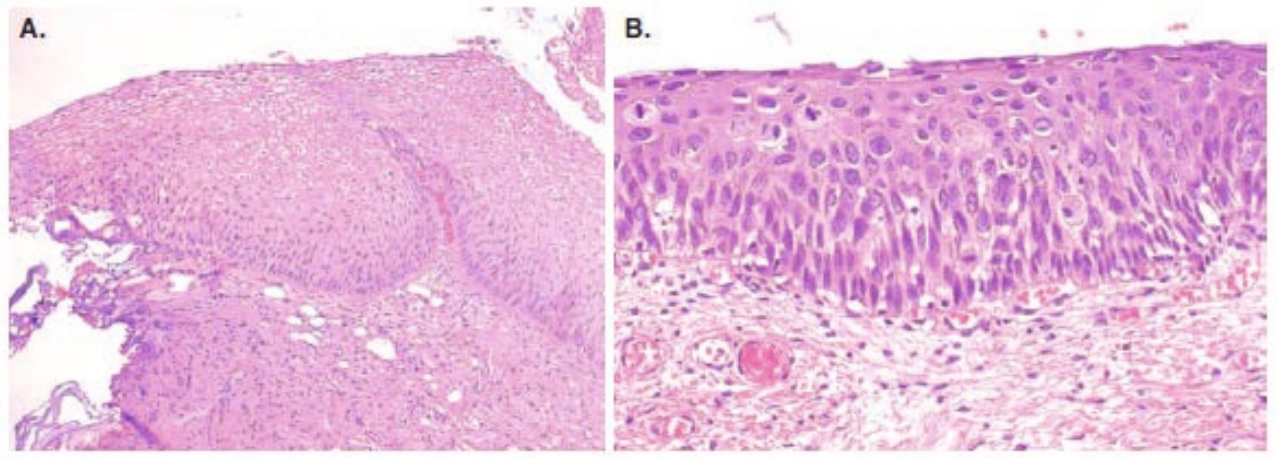

Figure 1. Histopathological findings in productive and transforming HPV infections

A. productive HPV infection, characterized by koilocytosis and other minor cellular reactions. B. CIN 3 lesion, characterized by an increased number of mitotic figures, actively replicating cells throughout the thickness of the epithelium and an increased nucleus to cytoplasm ratio. Both figures are hematoxylin and eosin stained. Adapted from Litjens et al. (86)

\section{Transforming infection}

High-grade intraepithelial neoplasia of the cervix occurs when the normal viral gene expression is deregulated. $(28,29)$ Expression of E6 and E7 in the basal cell layers is increased, initiating uncontrolled cell proliferation and immortalization and making the cell susceptible to chromosomal instability. This type of infection is known as a 'transforming infection' and is associated almost exclusively with high-risk HPV types. A transforming infection is the basis for cervical oncogenesis. Transforming infections are cytologically and histologically characterized by increased numbers of mitotic figures, actively replicating cells throughout the thickness of the epithelium and an increased nucleus to cytoplasm ratio, which are diagnosed as high-grade CIN (figure 1). Several hypotheses have evolved as to the origin of the cervical transforming infections: they could be the result of a persisting and deregulating productive infection or originate as primary transforming infections. An interesting new concept was recently introduced by Herfs et al.(30) They found that the removal of squamocolumnar junction (SC]) cells decreased disease recurrence. This empowered a recently introduced hypothesis that specific SC] cells may be the origin of high-grade $\mathrm{CIN}$ and that these cells may not regenerate after excision. They showed that most high-grade $\mathrm{CIN}$ lesions developed in $\mathrm{SC}$ ] cells, whereas most low-grade CIN lesions developed in other cells populations, such as metaplastic epithelium of the cervix or the ectocervix. They concluded that SC] cells may be more susceptible to HPV infection and dysplastic changes. Following on this hypothesis, one may imagine two pathways to high-grade CIN: some highgrade CIN lesions occur as a result of a primary transforming infection of the S]C cells, whereas other high-grade $\mathrm{CIN}$ lesions may occur as a result of a persistent and deregulated productive infection of other cervical cells. This concept was recently elegantly illustrated by Steenbergen et al.(21) 


\section{Oncogenesis}

\section{Proliferation, immortalization and genomic instability}

Cervical carcinogenesis is characterized by a combination of uncontrolled cell proliferation and immortalization and genomic instability, for which the early viral genes E6 and E7 are largely responsible. The combined action of HPV E6 and E7 leads to cell proliferation and immortalization, through processes summarized in figure 2. Furthermore, it induces genomic instability, which is the result of deregulation of the centrosome cycle and direct DNA damage. As a result, a proliferating cell population will evolve with chromosomal aberrations.

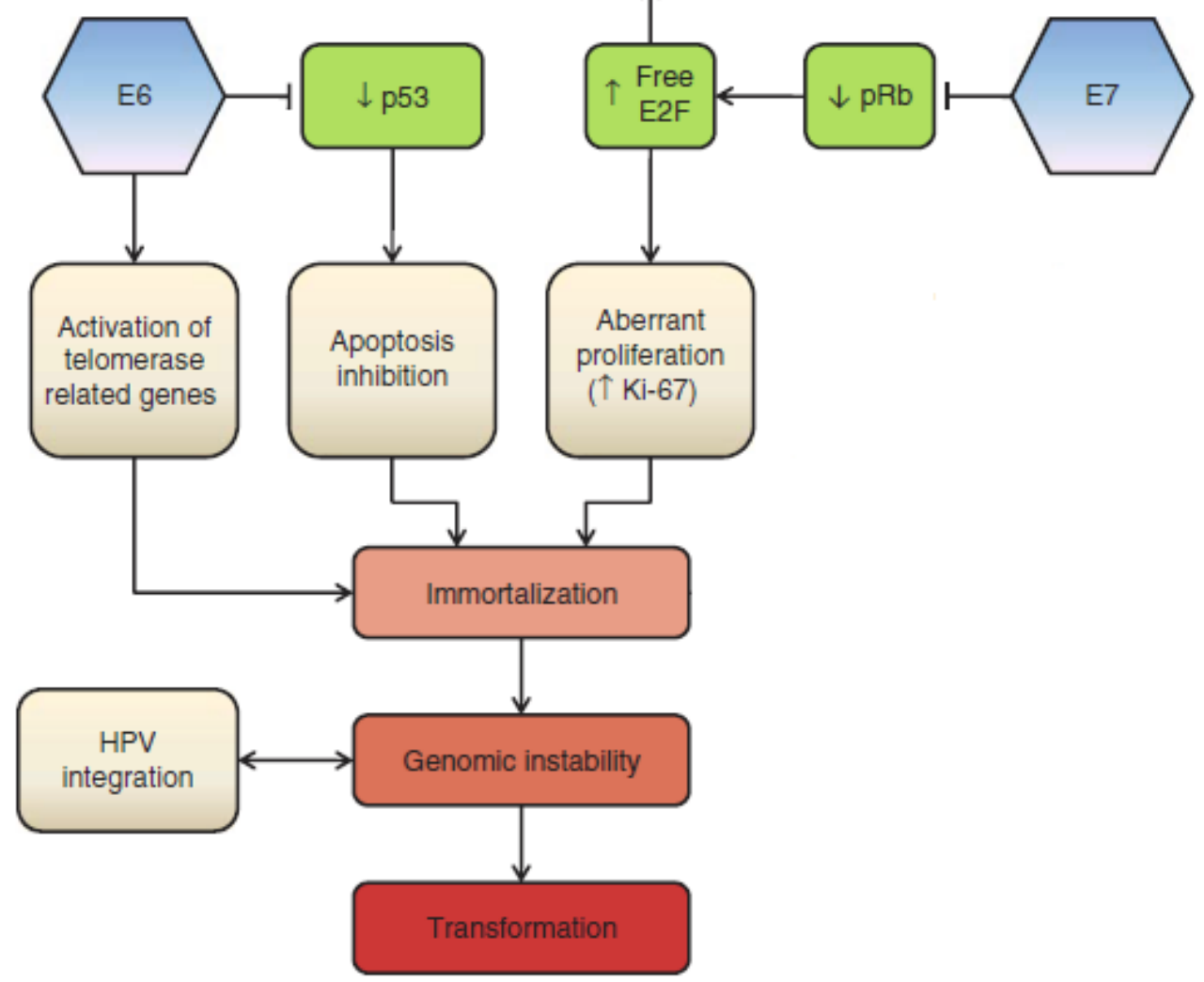

Figure 2. Cellular processes in cervical carcinogenesis following HPV infection.

E6 acts through inhibition of p53, a well-known inductor of apoptosis. Additionally, E6 leads to the increase of telomerase activity by activation of transcription of telomerase reverse transcriptase, leading to sustained telomerase, necessary for cell immortalization. E7 inactivates tumour suppressor protein retinoblastoma $(R b)$, thereby increasing free $\mathrm{E} 2 \mathrm{~F}$, leading to cellular proliferation. Cells in which the normal actions of $R b$ are suppressed by $E 7$ are characterized 
by an increased expression of proliferation markers, such as Ki67, and increased expression of p16. P16 is a protein that promotes cell cycle arrest through prevention of hyperphosphorylation of $R b$. However, its function is bypassed by HPV, because binding of $E 7$ to $p R b$ creates a phosphorylation independent release of E2F. The combined action of E6 and E7 proteins stimulate S-phase cell cycle entry and surpassing of cell cycle checkpoints, leading to cell proliferation and immortalization. With additional accumulation of genomic instability, this forms the basis for cervical cancer development. Adapted from Litjens et al (86)

\section{DNA integration}

DNA integration occurs frequently in cervical carcinogenesis. $(28,29)$ Disruption of the viral genome takes place at fragile sites, often in the E1 and E2 gene sites. Viral integration leads to loss of E2 expression, which is responsible for repression of early gene expression. As a result, expression of the E6 and E7 genes is enhanced, leading to cell proliferation. Furthermore, cells with integrated viral DNA show increased genomic instability and therefore have a greater probability of malignant progression. Interestingly, viral integration is not present in all HPV-induced cervical malignancies. Whereas almost all HPV-18 positive cervical malignancies contain integrated DNA, it is found in only approximately $70 \%$ of HPV 16 positive cervical malignancies. This indicates that viral integration may not to be essential for carcinogenesis and may be a consequence of the carcinogenic process rather than a cause.(31)

\section{Epigenetic changes}

Epigenetic changes in the host genome influence gene expression and disease control in many diseases. Although the exact mechanisms are not yet completely understood, the role of epigenetic modification by HPV in cervical oncogenesis has been established. $(21,29,32)$ HPV induces modifications to the host DNA methylation status and aberrant miRNA expression, which results in silencing of tumour-suppressor genes and the overexpression of oncogenes. Recently, it has been suggested that methylation analysis may distinguish advanced high-grade CIN from those with a high probability of spontaneous regression.(21) This is understandable in view of the fact that accumulation of genetic and epigenetic changes in the host and/or viral genome are acknowledged to be involved in progression toward invasive cervical cancer. Methylation of viral DNA may also contribute to cervical oncogenesis. Methylation occurs at $\mathrm{CpC}$ sites and this process is thought to induce a transforming infection, leading to high-grade CIN.(33)

\section{Natural history of high-grade CIN: what may influence disease regression or progression?}

In conclusion, cervical intraepithelial neoplasia is the result of transforming infections by HPV, in which expression of E6 and E7 is deregulated in the basal cell layers. Oncogenesis is further aided by genomic instability, viral DNA integration and epigenetic modifications. However, only a minority of HPV infections persists and may result in high-grade $\mathrm{CIN}$. Of these, only a subset will progress to invasive cervical cancer. As mentioned before, the natural history of HPV infection and $\mathrm{CIN}$ lesions is determined by a complex interaction between viral and host factors (figure 3). The immune system must ensure clearance of the infected cells before the effects of E6 and E7 in different important cellular functions will result in a CIN lesion that can no longer regress. Understanding of these influencing factors is essential for the potential identification of those 
individuals in which HPV infections and CIN will and will not persist. An important viral factor is the individual oncogenic potential of different HPV types, which is determined by differences in interactions with cellular functions after infection. For example, the affinity of E6 and E7 for p53 and $\mathrm{pRb}$ is lower for low risk-HPV types than for high-risk HPV types. Other important viral and host factors influence the ability of the infected individual to clear HPV infection or HPV-induced $\mathrm{CIN}$ lesions. These include the ability of HPV to evade and down regulate the host immune system and also the individual capacity of the host immune system for clearance of HPV and CIN lesions. Cenetic differences in immune capacity, acquired immunodeficiency and other factors such as smoking may lead to an increased or decreased individual ability for clearance.

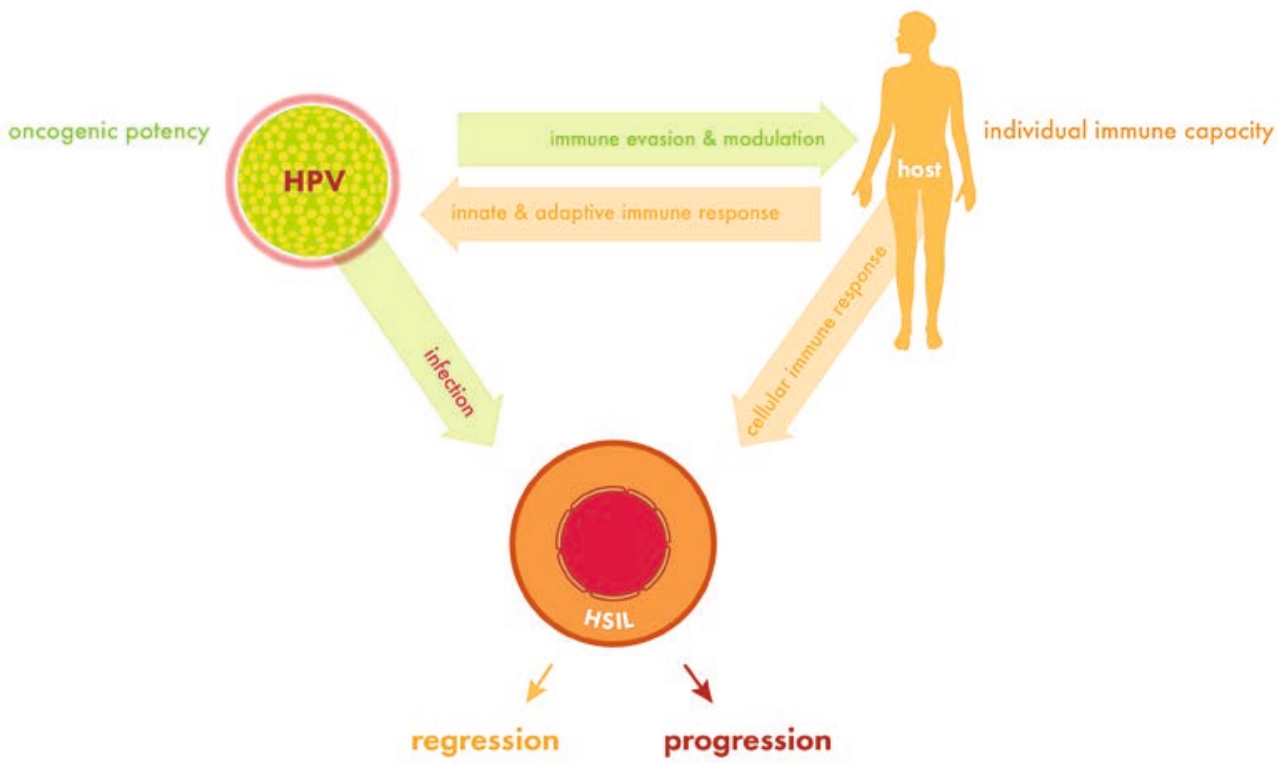

Figure 3. Interaction between virus and host in the natural history of high-grade CIN.

The natural history of high-grade CIN is influenced by a complex interaction between viral and host factors. The most important established viral factor is the HPV genotype, determining the viral oncogenic potential. Host factors include factors which influence the individual immune capacity, such as genetic differences in immune capacity and acquired immunodeficiency. The attributing role of the immune response in the natural history of HPV infection and CIN lesions will be reviewed hereafter.

\section{Immune response}

The immune response to HPV infection has been extensively reviewed by other authors.(34, 35) An overview of important immunological mechanisms will be provided here as a basis for biomarker selection. 
HPV infection and HPV induced lesions can both be cleared by a combination of the innate and adaptive immune system. Controversy exists as to whether HPV infections are truly cleared or just become latent as a result of the host immune response.(36) Whereas the innate immune system acts mainly as an immune barrier to HPV infection, the cellular immune response of the adaptive immune system is responsible for clearance of HPV-induced lesions of the cervix. The humoral immune response to HPV infection is a slow and inconsistent process: only $50-70 \%$ of those infected show seroconversion. The humoral immune response is believed to play no part in lesions clearance, but seems to be protective against new infections.

The immune response to HPV and HPV induced lesions is a largely local process and relies on the individuals immune capacities, but is also influenced by characteristics of the virus and the infectious process: HPV is characterized by several immune evasion strategies and infection leads to active downregulation of immune responses.(34)

Several viral characteristics lead to immune evasion. Infection with HPV is a largely intraepithelial process with limited viraemia to activate the immune response. In addition, replication of HPV in the differentiating keratinocyte does not lead to cytolysis or cell death and an associated inflammatory reaction. This results in an impaired activation of both the innate and adaptive immune systems. Beside immune evasion, HPV also causes downregulation of immune responses atseveral stages. HPV inhibits interferon synthesis and signalling and dampens cytokine responses, down regulating both the innate and cellular immune response to infection. Furthermore, HPV seems to impair the normal function of several innate immune cells. Langerhans cells are not activated by the uptake of HPV capsids. Instead, dermal dendritic cells (DCs) may be the key players in the HPV recognition and initiation of the cellular immune response. NK cells show reduced cytotoxic activity and their cell receptor expression seems to be reduced in the presence of HPV. The combination of immune evasion and downregulation of the immune system enables HPV to reside in the host epithelium for a long time with limited immune response, leading to alteration of cellular processes as previously described with neoplastic progression as a result.

\section{Immune response in persistent HPV infections}

In most women, the immune response is able to clear HPV infection and induce regression of low grade $\mathrm{CIN}$, in spite of the immune evasion and downregulation by HPV. Persistent HPV infection is present in a smaller subset of women, influenced by a combination of host and viral factors. These women are at risk for the development of high-grade $\mathrm{CIN}$ and cervical carcinoma. With neoplastic transformation and genomic instability, key mediators of the immune system such as cytokines, adhesion molecules, chemokines and their receptors are further deregulated. There is evidence for an impaired cell-mediated immune response in women with high-grade CIN. Activation of the adaptive immune system leads to differentiation of CD4+ lymphocytes into T-helper cells, which produce cytokines of two different profiles: Th-1 cytokines promote differentiation of CD8+ lymphocytes into CTLs, which stand at the base of the cell-mediated immune response to HPV infected cells. Th-2 cytokines induce antibody production, as part of the humoral immune response. In cervical neoplasia, increasing CIN grade is associated with a shift in 
cytokine profiles. Low grade CIN is characterized mainly by cytokines of the Th-1 profile, whereas high-grade $\mathrm{CIN}$ and cervical carcinoma are characterized by Th-2 profile cytokines, indicating a shift from cell-mediated to humoral immune response with increasing CIN grade. $(37,38)$ Finally, the cellular immune response seems to be further deregulated by regulatory T-cells. Increased frequencies and suppressive activity of these cells has been found in patients with high-grade $\mathrm{CIN}$ and cervical carcinoma, indicating an active downregulation of the immune response in more progressive disease.(39)

In conclusion, the development of high-grade $\mathrm{CIN}$ is the result of a persistent and transforming HPV infection. A subset of high-grade CIN can be cleared by the host cellular immune response (figure 4). The likelihood of spontaneous regression is determined by the balance between HPVinduced effects (deregulation of cellular processes and downregulation of the immune response) and the capacity of the host immune system.

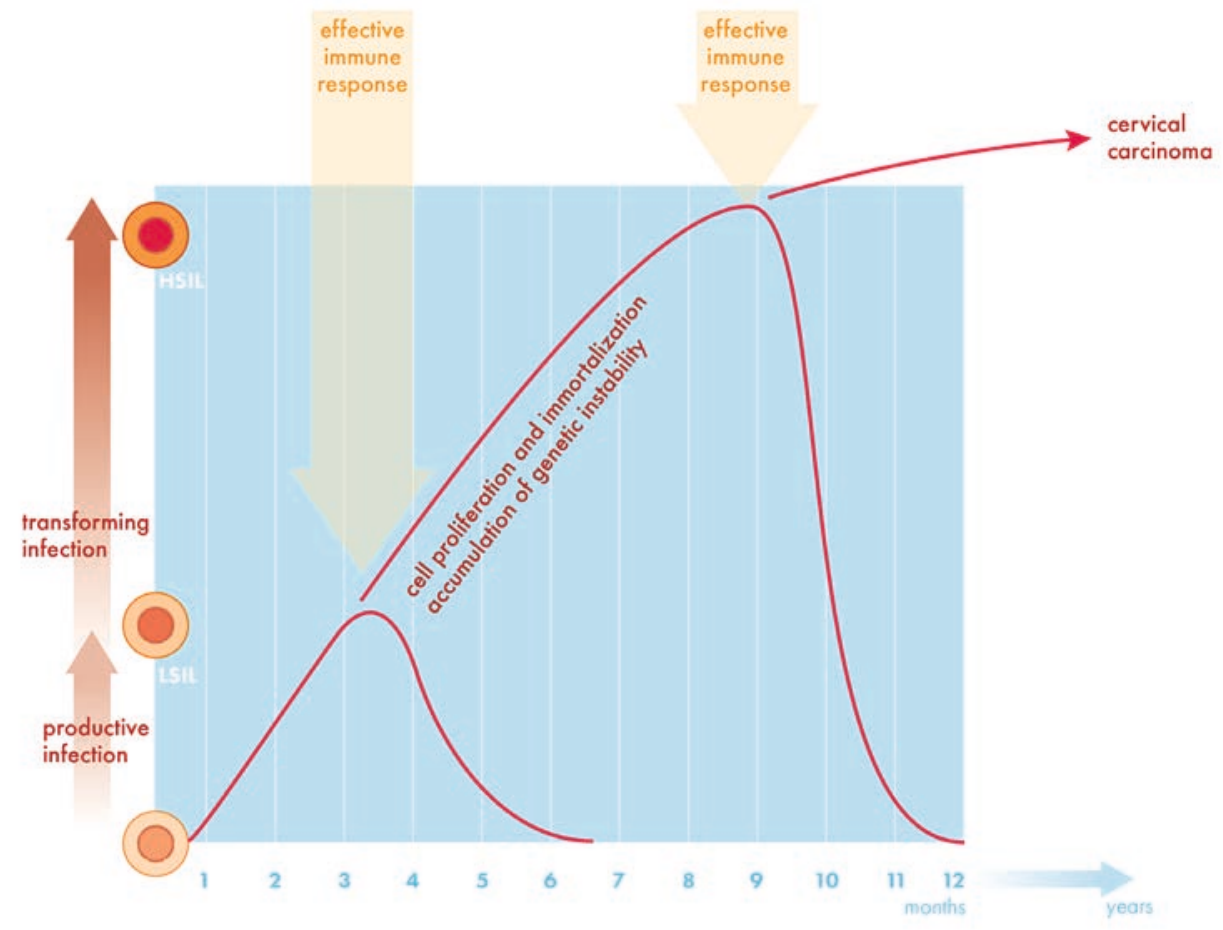

Figure 4. Natural history of CIN in the course of time.

The development of high-grade CIN is the result of a persistent and transforming HPV infection, in which HPVinduced cell proliferation and immortalization and the accumulation of genetic instability cause histopathological abnormalities. Whereas the development of CIN is a relatively short-term process, the subsequent development of cervical carcinoma takes many years. Most low-grade lesions and a subset of high-grade CIN can be cleared by the host cellular immune response at any point of time during the infectious process, as indicated at two stages in this figure. 


\section{Identification of candidate prognostic biomarkers in the natural history of high-grade CIN}

We aimed to identify biomarkers that predict the natural history of histologically confirmed high-grade $\mathrm{CIN}$ (CIN 2-3). These must be biomarkers that predict spontaneous regression or progression of high-grade $\mathrm{CIN}$. To this end, a search for promising biomarkers was conducted and a critical appraisal was performed as described below.

\section{Search strategy}

A search was conducted on Pubmed using combinations of the following search terms: "cervical intraepithelial neoplasia"[MeSH Terms]; "biological markers"[MeSH Terms] OR ("biological"[All Fields] AND "markers"[All Fields]) OR "biological markers"[All Fields] OR "biomarker"[All Fields]; "natural history"[MeSH Terms] OR ("natural"[All Fields] AND "history"[All Fields]) OR "natural history"[All Fields]; "disease progression"[MeSH Terms] OR ("disease"[All Fields] AND "progression"[All Fields]) OR "disease progression"[All Fields] OR "progression"[All Fields] "regression"[All Fields]; ("humans"[MeSH Terms] OR "humans"[All Fields] OR "human"[All Fields]) AND ("papillomaviridae"[MeSH Terms] OR "papillomaviridae"[All Fields] OR "papillomavirus"[All Fields]). Language was restricted to English only. Those studies were selected that investigated prognostic biomarkers in histologically confirmed CIN lesions. Publications were first selected based on title and abstract. Final inclusion or exclusion was based on review of the full publication content. Additionally, relevant references from selected articles were also evaluated for inclusion.

\section{Overview of candidate prognostic biomarkers in the natural history of high-grade CIN}

As reviewed above, persistence or regression of HPV-induced lesions is determined by a complex interaction between the immune system, viral factors and different cellular functions. Understanding of the underlying biological mechanisms provides a rational basis for the selection of biomarkers with a clear role in cervical carcinogenesis and provides an extensive amount of candidate biomarkers. $(40,41)$ Candidate prognostic biomarkers of high-grade CIN can be categorized into three main areas: host factors, viral factors and cellular factors and will be discussed accordingly.

\section{Host factors}

Human Leukocyte Antigens (HLA) subtypes

Many viral infections are controlled by T-cells recognizing their antigen within the context of HLA class I and II molecules expressed on the cell surface. Evidence suggests a role for HLA polymorphisms in the individual risk of cervical cancer after HPV infection, based on case-control studies.(42) Two authors found an association between a certain HLA subtype and disease progression in histologically confirmed low-grade CIN. Matsumoto et al. found a protective effect of HLA-DRB1 1302 against progression of $\mathrm{CIN}_{1 / 2}$ to $\mathrm{CIN}_{3}$ in 454 patients. (43) Sastre-Carau et al. found an association between $\mathrm{HLA}-\mathrm{DRB} 1$ * 13 and regression of $\mathrm{CIN} 1$ in a cohort of 86 women with $\mathrm{CIN}$ 1. Disease regression was $71.8 \%$ in patients with HLA-DRB1" 13 and $45.9 \%$ in patients with other genotypes. Regression reached $90.5 \%$ (38.9-98.5\%) at 18 months in DRB1*13 patients with 
HPV16/18-negative-associated CIN.(44) One study was conducted in patients with histologically confirmed high-grade CIN. Trimble et al. found a decreased disease regression of non-HPV16 high-grade $\mathrm{CIN}$ in the presence of HLA*A201 in 100 patients. Disease regression was $14.3 \%$ for patients with non-HPV16 high-grade CIN who carried an HLA*A201 allele, compared to $42.3 \%$ in those without an HLA*A201 allele.(8) HLA*A201 is expressed by approximately half of the population. The authors hypothesise that the effect of HLA polymorphisms could be explained by either differences in efficiency of antigen presentation or an altered immune response, in which differences in generation of regulatory $\mathrm{T}$ cells and/or NK cells occur.

\section{Lymphoproliferative response}

Multiple components of the cell-mediated immune response have been studied as potential biomarkers in the natural history of $\mathrm{CIN}$ and several promising biomarkers have been identified. As part of a composite biomarker profile containing Rb, lesions size, $\mathrm{CD} 4+$ cells and condom use, Munketal. established an association between lower numbers of CD4+cells in the basal membrane and disease regression, in 162 women with histologically confirmed high-grade CIN during a median interval of 16 weeks.(6) Spontaneous regression occurred in $21 \%$ and was significantly increased in smaller lesions with increased $\mathrm{Rb}$ expression and a lower number of stromal $\mathrm{CD}_{4}+$ cells (regression in 53\%). No correlation was found between disease regression and epithelial and stromal CD8+, CD138+ and CD25+ cells. The authors hypothesize that a lower number of stromal $\mathrm{CD} 4+$ cells, as a marker of T-helper cells, may reflect a more effective immune response: HPV immune modulation leads to a disturbed immune response with on-going activation of innate immune cells. Ovestad et al. retrospectively studied the local immune response in 55 patients with histologically confirmed CIN 2/3, which were followed for a mean of 12 weeks (5-34 weeks). (45) Assessment of helper T-cells, cytotoxic T-cells, regulatory T-cells and B-lymphocytes was done by quantification of CD4, CD8, CD 25, Foxp3 and CD 138. Regression occurred in $18 \%$. After multivariate survival analysis, disease regression was independently predicted by higher stromal CD8+ cell numbers, higher $C D 4+/ C D 25+$ cell ratio and lower stromal CD $138+$ cell numbers, indicating a strong T-cell response. None of the HPV16-positive cases showed regression. Interestingly, HPV16 infection correlated with lower CD8+ cells and higher CD 25+ cells, indicating a potentially stronger immune modulatory effect of HPV16 than other types. Moreover, stromal CD25+ cells (possible regulatory T cells) were more common in persistent cases, indicating that the specific immune response may be suppressed in these cases. The association between local regulatory $T$ cells and disease persistence or progression was also established by Kojima et al., in patients with low grade cytological abnormalities.(46) They evaluated 12 patients with regressing $\mathrm{CIN} 1 / 2$ and 12 patients with persisting $\mathrm{CIN} 1 / 2$ during 8-33 months and found significantly lower numbers of regulatory $\mathrm{T}$ cells in cytobrush material of $\mathrm{CIN}$ regressors as compared to non-regressors. Molling et al. studied the presence of systemic regulatory T cells (PMBCs) in 82 women with HPV16 positive low grade cytological abnormalities.(47) The frequency of regulatory T cells was significantly increased in women with persistent HPV16 infections, which may lead to an inefficient immune response and may contribute to subsequent progression to neoplasia. However, they found no differences in the presence of systemic regulatory T cells between patient who did and did not progress to $\mathrm{CIN} 3$ during prospective follow-up. In conclusion, markers of local immune response have been shown to be associated with disease regression, although the 
available evidence lacks consistency. As clearance of high-grade $\mathrm{CIN}$ is effected by the individual immune response, using markers of this response as prognostic biomarkers seems sensible. More research is required for application purposes.

\section{Telomerase amplification}

Telomerase is an enzyme complex that provides telomere maintenance, a process that is normally restricted by activation of $\mathrm{pRb}$ and $\mathrm{p} 53$. Oncogenesis is associated with increased telomerase activity. Telomerase consists of several subunits, of which the human telomerase gene (hTERC) serves as a template during telomere elongation. The amplification of hTERC can stop cell apoptosis, leading to tumour occurrence. Gain of hTERC has been shown to correlate with histological disease grade.(48) Only one author conducted a prospective study, evaluating the prognostic value of telomerase amplification in CIN. Lan et al. studied 54 patients with histologically confirmed $\mathrm{CIN} 1 / 2$, during 24 months. All lesions were associated with low-risk HPV types. All patients that showed progression to CIN 3 displayed gain of hTERC, whereas progression did not occur in patients were hTERC had not been amplified.(49) As such, hTERC amplification may have diagnostic purposes, in differentiating between low-grade CIN lesions with low or high risk of disease progression. Given its clear and established role in carcinogenesis and its association with $\mathrm{CIN}$ grade, its function as a prognostic biomarker for the natural history of CIN deserves further research.

\section{Other markers}

Both HPV antibodies and cytokine profiles, as markers of the host immune response, have been associated with disease grade in $\mathrm{CIN}$, making them potential diagnostic biomarkers. $(38,50)$ The evidence on their value in disease prognosis is limited, however. One author found a significantly higher regression rate of $\mathrm{CIN}$ in patients with a stronger Th-1 cytokine response.(51) The population size was limited, however, and disease severity was not documented. The evidence on HPV antibodies in the prognosis of CIN is limited and conflicting and does not identify HPV antibodies as a promising biomarker. One author found an association between IgC positivity and progression to $\mathrm{CIN}_{3}$ in patients with HPV16 positive cytological abnormalities. (52) Another

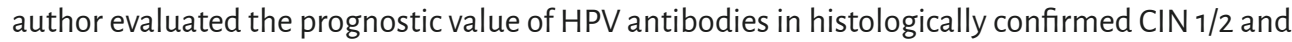
found no correlation between baseline IgC reactivity to HPV L1 capsid and disease regression.(53)

The role of HPV induced epigenetic changes in cervical carcinogenesis is receiving increasing attention. Both altered microRNA expression and host DNA methylation is found in CIN. The mechanisms by which HPV oncoproteins deregulate microRNA expression and associations between several microRNAs and cervical cancer are reviewed by others. $(54,55)$ MicroRNAs have not yet been proven useful as diagnostic or prognostic markers in cervical carcinogenesis, but deserve more research in the future. Host DNA methylation of several sites has been identified as a diagnostic biomarker in CIN.(56-58) Moreover, it has recently been postulated that the extent of DNA methylation may distinguish CIN lesions with high short-term progression risk from those with low risk, as more extensive methylation may reflect a longer existing, transforming infection instead of a productive infection.(21) 
Although current evidence is limited, DNA methylation seems to be a promising prognostic biomarker and deserves more research in the future.

\section{Viral factors}

\section{Viral genotype}

The influence of HPV subtype on the risk of CIN development and progression has been established many years ago, leading to categorization of HPV into high-risk and low-risk types. (59) An increased risk for CIN progression was found for HPV16, -18, -31, -33, -52 and -58.(6062) HPV16 has been associated with a decreased rate of spontaneous regression. Trimble et al. followed 100 women with $\mathrm{CIN} 2 / 3$ over a mean of 15 weeks.(8) Spontaneous regression occurred in $20.5 \%$ of those with single HPV16 infection as compared to $36.4 \%$ of those with an infection other than HPV16. Ovestad et al. followed 55 patients with histologically confirmed CIN 2-3 over a mean follow-up period of 12 weeks. $18 \%$ regressed, of which none contained HPV16. All 18 cases with HPV16 persisted.(7)

\section{Viral DNA methylation}

Clarke et al. reviewed the literature on viral DNA methylation in CIN diagnosis and concluded that the detection of methylation of $\mathrm{CpC}$ sites in the L1 gene may distinguish between HPVinfected women with $\mathrm{CIN} 2+$ and without CIN.(33) The authors state that it is not yet clear whether methylation of HPV DNA reflects an early event that can be measured as a prognostic biomarker of later $\mathrm{CIN} 2+$, or whether it is a late event that will serve only as a diagnostic biomarker. The evidence on the prognostic value of viral DNA methylation is limited. Mirabello et al. evaluated the prognostic value of DNA methylation in pre-diagnostic samples of $\mathrm{CIN}_{2}+$ lesions and found good performance of methylation of $\mathrm{CpC}$ sites in the $\mathrm{L} 1$ and $\mathrm{L} 2$ gene as prognostic markers for the development of $\mathrm{CIN} 2+$.(63) Oka et al. is the only author that studied an association between viral DNA methylation and the natural history of histologically confirmed CIN. 15 patients were followed (10 patients with $\mathrm{CIN} 1,4$ patients with $\mathrm{CIN} 2$ and one patient with $\mathrm{CIN} 3$ ). Eight cases showed progression: all eight cases were $\mathrm{CIN}_{1} / 2$ and progressed to $\mathrm{CIN}_{3}$. The only case with $\mathrm{CIN}$ 3 regressed. Methylation rates of $\mathrm{L} 1$ gene were significantly higher in the progression group than in the regression group.(64)

\section{Other factors}

The HPV L1-gene encodes for the virus capsid protein. It represents about $90 \%$ of total protein on the surface of the virus and is typically evident during the reproductive phase of the HPV infection. (65) Several authors have found a correlation between loss of L1 capsid protein expression and disease grade. $(65,66)$ This seems logical, as L1-expession occurs in differentiating desquamating cells in the productive viral life cycle. A potential prognostic value of L1-expression in the natural history of CIN has been subject of study by several authors. Choi et al. found a positive association between $\mathrm{L} 1$ expression and disease regression of histologically confirmed $\mathrm{CIN}$ 1: the regression rate in HPV-L1 positive patients was $72,7 \%$ vs $38,1 \%$ in $\mathrm{L} 1$ negative patients. Progression did, however, occur in $L 1$ positive patients and L1 negative patients also showed regression.(67) Rauber et al. followed 279 women with histologically confirmed CIN $1 / 2$ during a mean of 25 months. Progression to $\mathrm{CIN} 3$ was found in both $\mathrm{L} 1$ positive and $\mathrm{L} 1$ negative patients, but progression 
occurred significantly more often in HPV L1 negative patients than in L1 positive patients $(25.9 \%$ vs $9.4 \%$ ). The positive predictive value for no progression to a higher disease grade was $90,6 \%$ in L1 positive women. The negative predictive value for progression in L1 negative women was $26 \%$. No subgroup analysis for patients with CIN 2 was made.(68) Sarmadi et al. followed 65 women, 43 with histologically confirmed LSIL and 22 with HSIL during 24 months.(65) Disease regression was significantly less frequent in L1 negative LSIL patients (33.3\%) than in L1 positive patients (60.7\%), while disease progression was more frequent. The sensitivity of L1 positivity for lack of disease progression in LSIL was 70,2\%, the specificity $100 \%$ and the PPV $100 \%$. All cases of HSIL were L1-negative.The study by Sarmadi et al. shows that L1-expression is lost in high-grade CIN. This is a logical consequence of early gene overexpression and loss of late gene expression in highgrade CIN. L1 therefore does not seem to be a logical prognostic biomarker in the natural history of high-grade CIN.

Wang et al. and Kim et al. found an association between HPV viral load at baseline and progression of low-grade CIN. $(69,70)$ However, this may also reflect the development of a new high-grade lesion in addition to a low-grade lesion, instead of true progression of the low-grade lesion. Furthermore, an association was found between a decreasing viral load over time and regression of high-grade $\mathrm{CIN}$, but this study does not provide information on the prognostic value of baseline viral load.(8) Kadish et al. and Peng et al. found an association between T-cell epitopes in the HPV16 E7 proteins and disease regression of LSIL and HSIL, but both studies were performed in small populations and the effect sizes seem to be limited. $(71,72)$

Viral integration has been identified as a promising diagnostic biomarker in $\mathrm{CIN}$, but the evidence on its prognostic value is limited. Manawapat et al. found an association between increased viral integration and persistence of HPV16 infection, but not disease progression to CIN 3.(73) Li et al. showed that persistent virus infection and progression of LSIL was more common in cases with integrated HPV16 than in cases with episomal HPV16.(74) The study was performed in a very limited number of patients.

\section{Cell cycle regulatory factors}

Understanding of the cellular processes involved in cervical carcinogenesis provides a basis for biomarker selection. The HPV oncoproteins Rb and p53, tumour suppressor protein p16 and proliferation marker Ki67 are probably the most well-known markers of cellular processes in cervical oncogenesis. Several cell cycle proteins have been identified and implemented in CIN diagnosis and others have been identified as potential biomarkers in CIN diagnosis.(75) Much less evidence exist on potential prognostic biomarkers.

\section{$p 53$ and $R b / p R b$}

Decreased $\mathrm{Rb}$ was identified as a strong independent predictor of $\mathrm{CIN} 1 / 2$ progression by Kruse et al., in a prognostic study in 90 patients.(76) pRb was not found predictive for the progression of histologically proven LSIL in 101 cases by Quint et al., who also found no association with p53. (77) One author studied the prognostic value of pRb in high-grade CIN: Munk et al. followed 162 patients with CIN 2-3 during an mean of 16 weeks.(6) Increased pRb was the strongest predictor 
of lesions regression. Two authors studied the prognostic value of both p53 and pRb in HSIL and found consistent results. Baak et al. followed 28 patients with histologically confirmed HSIL during a mean of 8.2 months and found that lower p53 and pRb in the deeper half of the epithelium was associated with disease persistence.(78) The overall regression rate was $43 \%$. Lesions with low expression of both markers persisted in $16 / 20$ cases (80\%), whereas none of the eight lesions with high expression of both markers persisted. Ovestad et al. followed 55 patients with histologically confirmed CIN 2/3 over a mean of 12 weeks. Increased expression of pRb and p53 was associated with disease regression and proved to be an independent predictor of disease regression with multivariate analysis.(7)

\section{Ki67}

An association between Ki67 and progression of $\mathrm{CIN}_{1 / 2}$ was established by Kruse et al.(79) They followed 44 patients for a maximum follow-up period of 18.8 months. Progression occurred in $11 \%$. Test characteristics of their Ki67-staining method showed a sensitivity of $100 \%$, specificity of $56 \%$, positive predictive value of $23 \%$ and negative predictive value of $100 \%$. Overall, $61 \%$ of cases were correctly classified. The results were validated in a test set of another 35 patients an showed similar results. As was later reviewed by Baak et al., the prognostically strongest Ki67 features were the Ki67 stratification index (threshold 0.57) and percentage of Ki67 positive nuclei in the middle third layer of the epithelium (threshold 30\%).(18) The association between Ki67 and natural history of high-grade $\mathrm{CIN}$ was studied by Baak et al. A study with a limited number of patients $(n=28)$ found no association between Ki67 expression and regression of high-grade $\mathrm{CIN}$.(78) Given the high prognostic value of Ki67 in low-grade $\mathrm{CIN}$, additional research seems indicated to evaluate the prognostic value of Ki67 in high-grade CIN.

\section{Other markers}

Currently, the only biomarker that has been implemented in clinical practice is p16. Its use is recommended by the College of American Pathologists and the American Society for Colposcopy and Cervical Pathology, as part of the LAST project (Lower Anogenital Squamous Terminology project).(80) Evaluation of p16 is intended as an adjunct to morphologic assessment of biopsy specimens for differentiation between HSIL and a disease state mimicking HSIL, or to differentiate CIN 2 into a HSIL and LSIL category. As such, p16 has been implemented as a diagnostic biomarker. The literature on the prognostic value of p16 is inconsistent. Several authors have found an association between p16 staining and progression of LSIL.(81) Recently however, Quint et al. found no correlation between p16 and LSIL progression: they followed 238 patients with histologically proven LSIL during two years. Age and HPV genotyping were identified as predictive for LSIL progression, but not cell cycle markers (pRb, p53, Ki67 and p16).(77) Three authors evaluated the prognostic value of p16 in the natural history of HSIL and found inconsistent results. Omori et al. followed 52 CIN cases during two years. Regression occurred in 28 lesions, progression in 13 cases. The risk of progression was greater with stronger p16 expression and a higher frequency of a punctate nuclear signal.(82) Baak et al. followed $28 \mathrm{HSIL}$ patients during a mean of 8.2 months and found no association between p16 and disease persistence or regression.(78) Ovestad et al. retrospectively studied 55 patients for a mean of 12 weeks and found that p16 staining intensity in the upper epithelium was significantly stronger in the persistent cases. The conflicting results on 
the performance of p16 as a prognostic biomarker may be explained by the fact that it is, in fact, a surrogate marker of cell cycle processes in the development of CIN.

Lu et al. studied insulin-like growth factor II m-RNA-binding protein 3 (IMP3) as a prognostic biomarker in high-grade $\mathrm{CIN}_{\mathrm{N}} \mathrm{IMP}_{3}$ is normally expressed in embryogenesis and is also an oncoprotein that is expressed in malignant tumours. Lu et al. followed 710 patients with histologically confirmed CIN 2/3 during a mean follow-up of 44 days before surgical intervention. (83) 140 of these patients were IMP3 positive and 38 patients were diagnosed with invasive carcinoma after LEEP. All invasive carcinomas were IMP3 positive and no IMP3-negative patient was diagnosed with invasive carcinoma. $27 \%$ of IMP3 positive patients with $\mathrm{CIN} 2 / 3$ developed invasive carcinoma within the time frame of the study. The possibility of the carcinoma already being existent at the time of the initial biopsies (biopsy error) cannot be excluded. The role of $\mathrm{IMP}_{3}$ in $\mathrm{CIN}$ was not established before and requires additional research that should precede further evaluation of IMP3 as a prognostic biomarker.

Recently, Uleberg et al demonstrated that zinc finger protein 441 and phospholipase D6 can predict regression of CIN 2-3.(84) These two proteins were identified in a learning set of 20 cases (10 regression and 10 persistence cases) and validated in an independent test set of another 20 high-grade CIN lesions (10 regression and 10 persistence cases). All 20 cases were correctly classified. Zinc finger proteins are transcription regulators functioning as tumour suppressors. Phospholipase proteins are involved in several cellular processes, including cell proliferation and may function by inducing epigenetic changes. The role of both biomarkers in $\mathrm{CIN}$ has not been established before. In this study, supernatants of fresh biopsy samples were used to extract watersoluble proteins, which are otherwise degraded in formalin-fixed, paraffin-embedded biopsies. Application of these promising biomarkers therefore requires additional fresh biopsy samples, making clinical applicability less likely.

\section{Composite prognostic biomarker profiles}

Baak et al. and Kruse et al. have developed prognostic biomarker profiles, by which patients can be categorized according to probability of lesion regression. The authors followed 90 patients with CIN $1(n=25)$ or CIN $2(n=65) .(18,76)$ Progression occurred in $17 \%$ and was effectively predicted by Ki67 testing. Furthermore, CIN progressors showed decreased Rb, CK 13, CK 14 and involucrin, and increased p21 and p27 expression. Ki67 Sl90 and Rb in the deeper half of the epithelium were the strongest multivariate independent predictors of progression. Further evaluation showed that combined quantification of Ki67, Rb, CK 13 and CK 14 gives accurate information about the progression risk of $\mathrm{ClN}_{1} / 2$. They developed a composite profile of these four markers, which categorizes patients into several groups with probabilities of lesion regression varying from $<1 \%$ to $100 \%$ (figure 5). Munk et al. developed a predictive biomarker profile for high-grade CIN. They evaluated $162 \mathrm{CIN} 2 / 3$ patients over a mean of 16 weeks.(6) The overall regression rate was $21 \%$. The percentage of $\mathrm{pRb}$ in the lower epithelium, lesions size, the amount of CD4+ cells in the stroma and consistent condom use were independently associated with lesion regression. $A$ model consisting of these four markers categorized patients into a group with a low regression probability of $8 \%$ and a group with a high regression probability of $56 \%$. 
The profile was characterized by a sensitivity of $71 \%$, specificity of $85 \%$, PPV of $56 \%$ and NPV of $92 \%$.

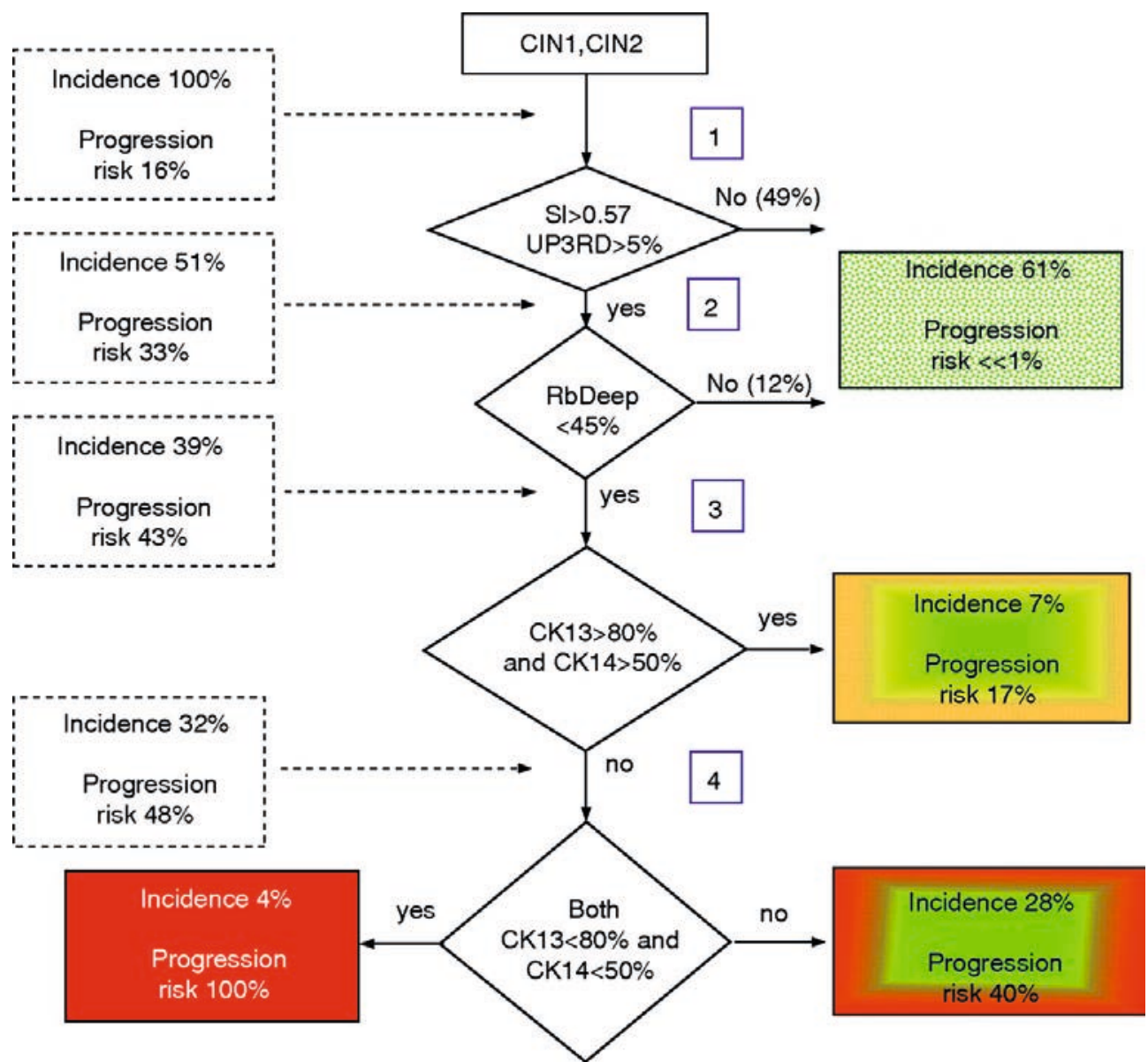

Figure 5. Prognostic biomarker based decision scheme of an early cervical intraepithelial neoplasia (CIN) lesion.

CK, cytokeratin; SI, Stratification Index; UP3RD, upper third. Diagnostic sections are stained with haematoxylin-eosin for routine evaluation and stained for $p 16$ to recognize CIN. A next serial section is stained with Ki67. Ki67 positive cells clusters are further analysed (step 1). If the Ki67 Sl90>0.57 or the percentage of Ki67-positive nuclei in the middle third layer of the epithelium exceeds $30 \%$ (which occurs in about $51 \%$ of cases), the likelihood of progression to $\mathrm{CIN} 3$ is about $30 \%$. A next serial section is stained for $p R b$ (step 2). If $p R b$-positive nuclei in the lower half of the epithelium $<40 \%$, progression risk is about $43 \%$. The progression risk of the remaining lesions is very low. Finally, a next section is stained for $\mathrm{CK} 13$ and $\mathrm{CK} 14$ (steps 3 and 4) and interpreted as shown in the figure. Based on all three analyses, patients can be divided into different progression risk groups, ranging from almost 0\% to $100 \%$. Adapted from Kruse et al. and Baak etal $(18,76)$ 


\section{Critical appraisal of candidate biomarkers}

All candidate biomarkers were evaluated according to two principles. First, we focus on those biomarkers with a clear role in the natural history of high-grade CIN. Second, their prognostic value and clinical applicability was evaluated according to established criteria. Pepe et al. have proposed a design to determine applicability of diagnostic and prognostic candidate biomarkers: the prospective-specimen-collection, retrospective-blinded-evaluation (PROBE) design.(20) They state that the development of a functional biomarker should go through several stages: discovery of the biomarker, determination of its classification accuracy and finally the validation of its impact on the prediction of clinical outcome. Their design consist of four key components: 1 . clinical context and outcome; 2. biomarker performance criteria; 3. biomarker test characteristics and 4. study size. For a more detailed information on their criteria, we refer to their publication on this subject. The majority of potential biomarkers in CIN have been evaluated for diagnostic and not for prognostic purposes.(75) Those studies that were performed on potential prognostic biomarkers in cervical precancerous lesions were mainly conducted in patients with low-grade $\mathrm{CIN}$ or cytological abnormalities as opposed to patients with high-grade CIN. Nevertheless, a substantial number of studies have been identified that evaluated biomarkers in the natural history of high-grade CIN and were discussed above. An overview all identified prognostic biomarkers in $\mathrm{CIN}$ can be found in table 1, which also provides a concise summary of their evaluation.

None of the studies were performed according to the PROBE criteria for determination of applicability of diagnostic and prognostic candidate biomarkers. Nonetheless, several biomarkers have been identified that show an association with the natural history of histologically proven high-grade $\mathrm{CIN}$ or have been identified as promising in this regard: they are associated at least with the natural history of low grade CIN or cytological abnormalities or are recently discovered biomarkers that warrant further investigation in their role as potential prognostic biomarkers. Of special interest are HLA subtypes, markers of the lymphoproliferative response and telomerase amplification (reflecting host factors in the natural history of $\mathrm{CIN}$ ), viral genotype and viral DNA methylation (reflecting viral factors in the natural history of $\mathrm{CIN}$ ), and Ki67, p53 and pRb (reflecting cellular factors in the natural history of CIN). HLA subtypes influence the individual immune response and could therefore influence the individual capacity for clearance of HPV and HPV-induced cervical lesions. One HLA subtype was identified in a prospective study, that was associated with decreased disease regression of high-grade CIN. This subtype is carried by approximately half of the population. Individual differences in immune response to HPV infection and cervical precancerous lesions can also be reflected in differences in the lymphoproliferative response. HPV induced lesions are cleared by the cellular immune response. Indeed, markers of a stronger T-cell response have been associated with disease clearance, whereas increased regulatory T-cells (acting as immune suppressors) have been associated with disease persistence. 


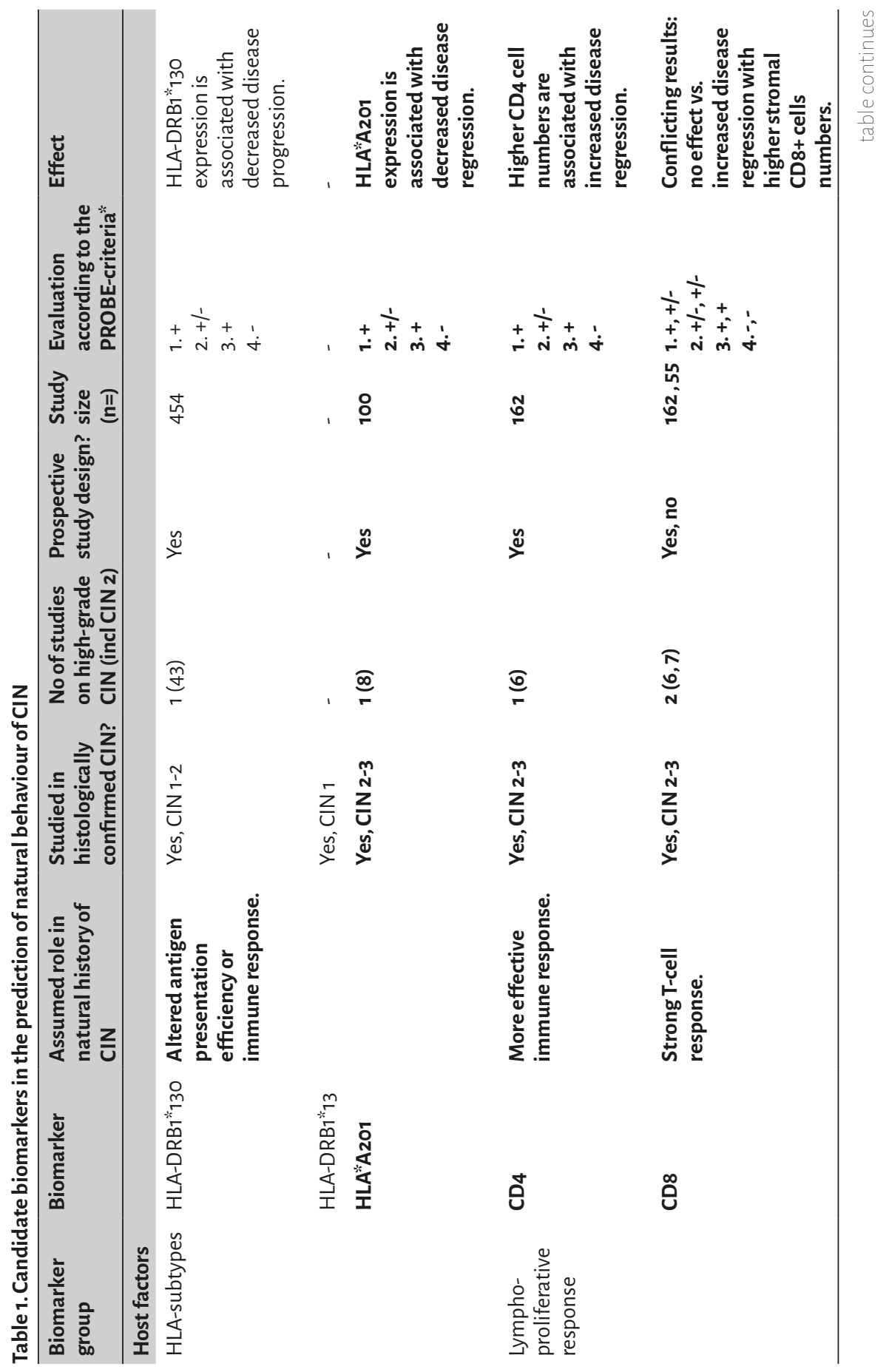




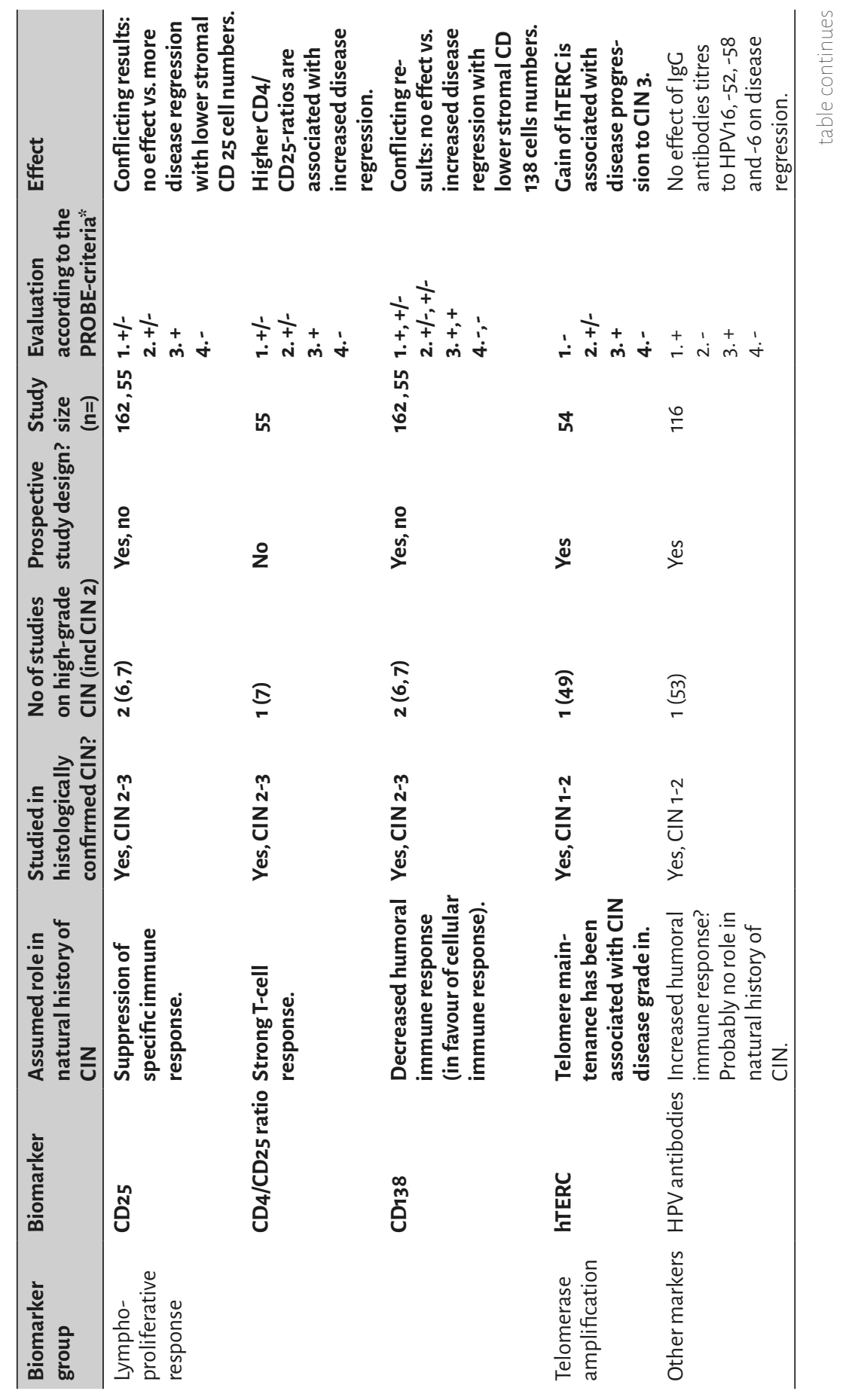




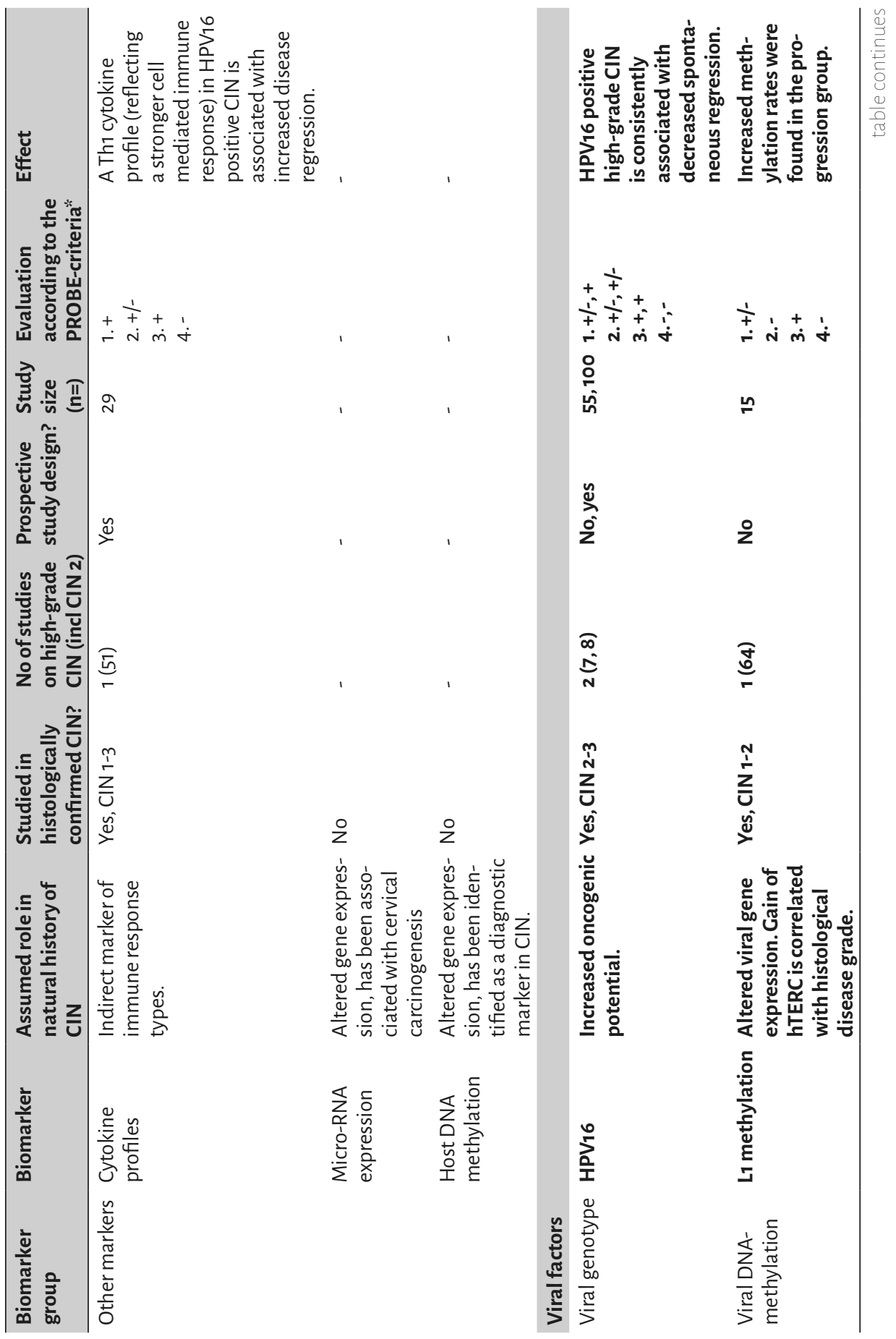




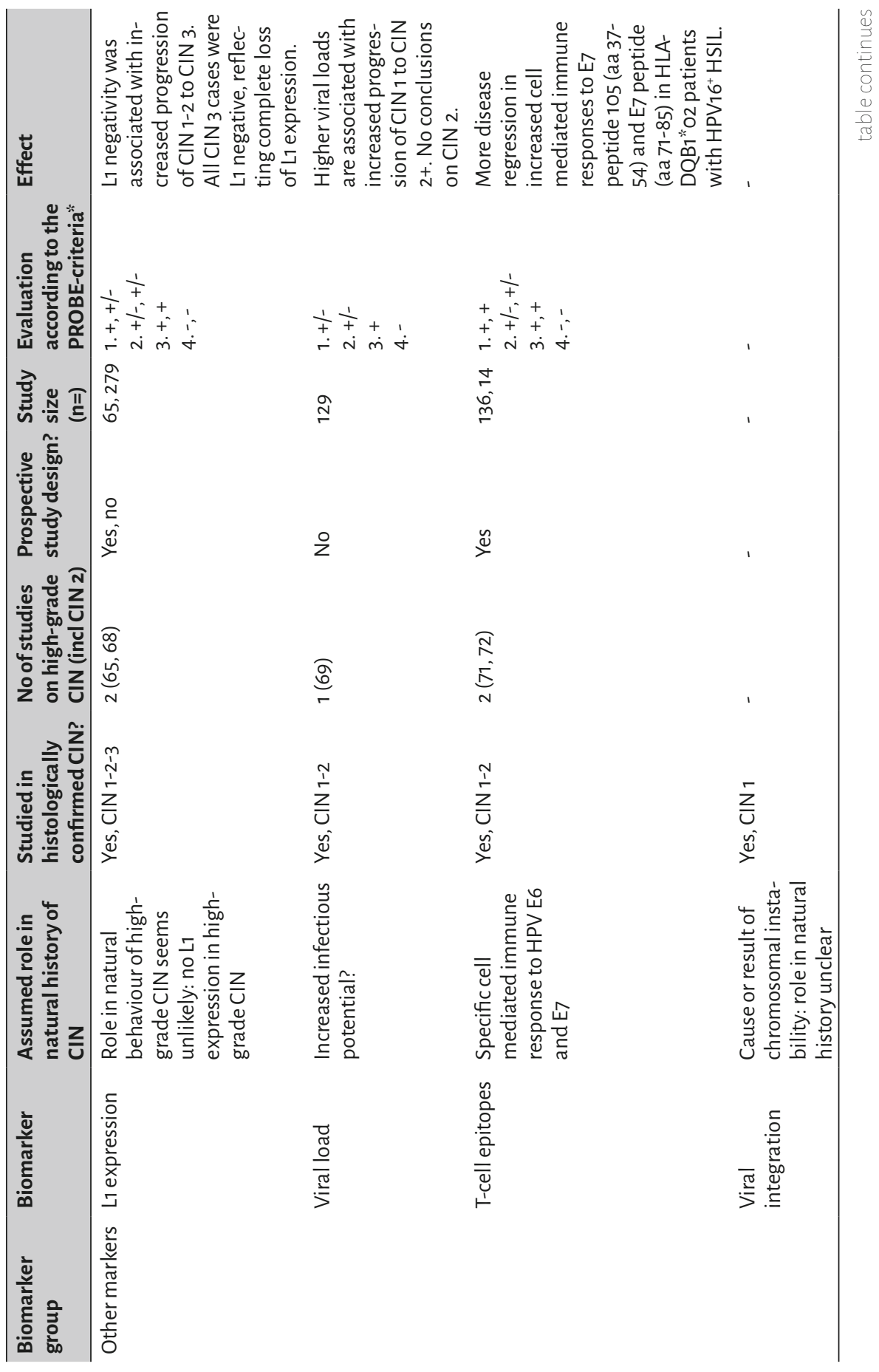




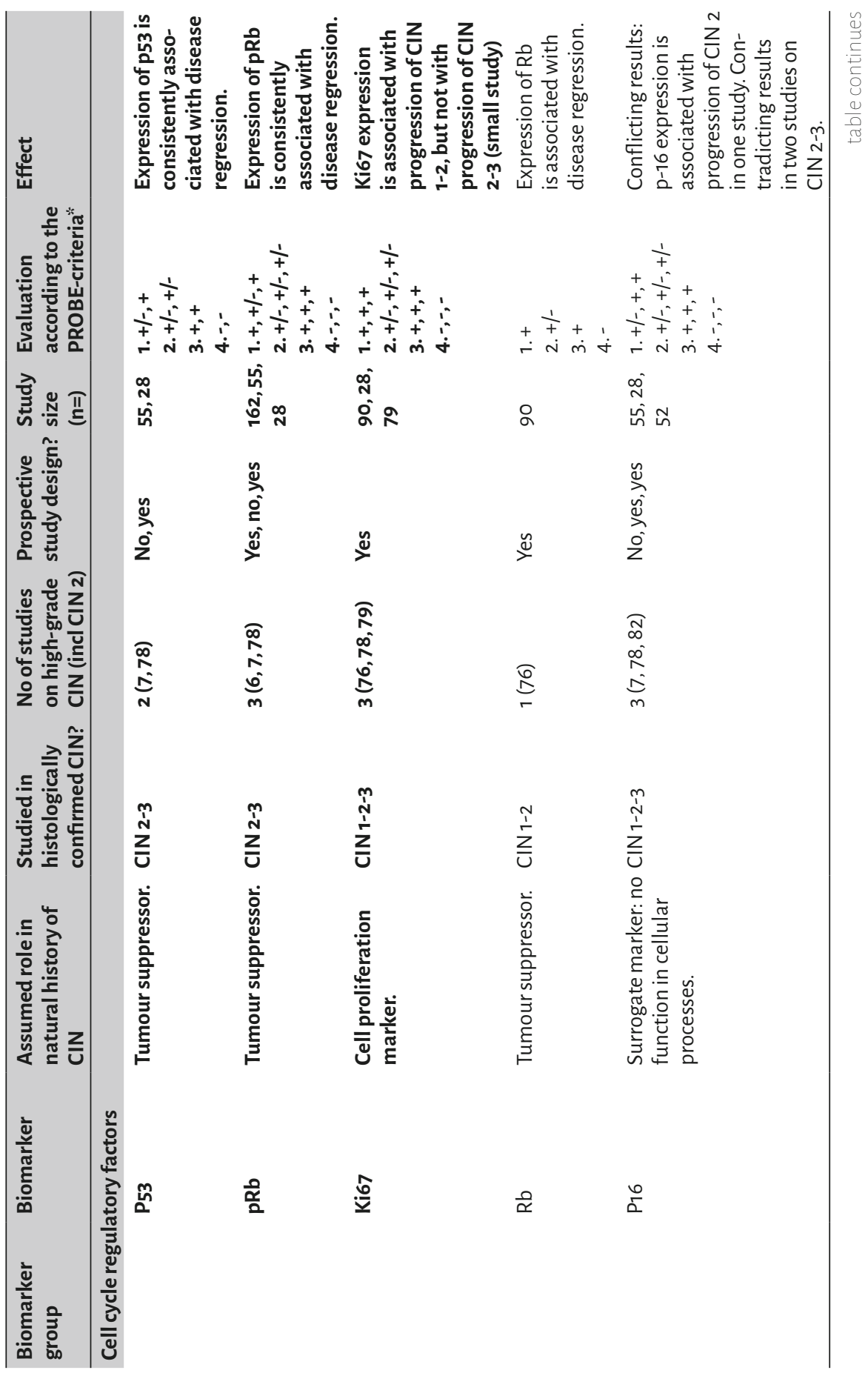




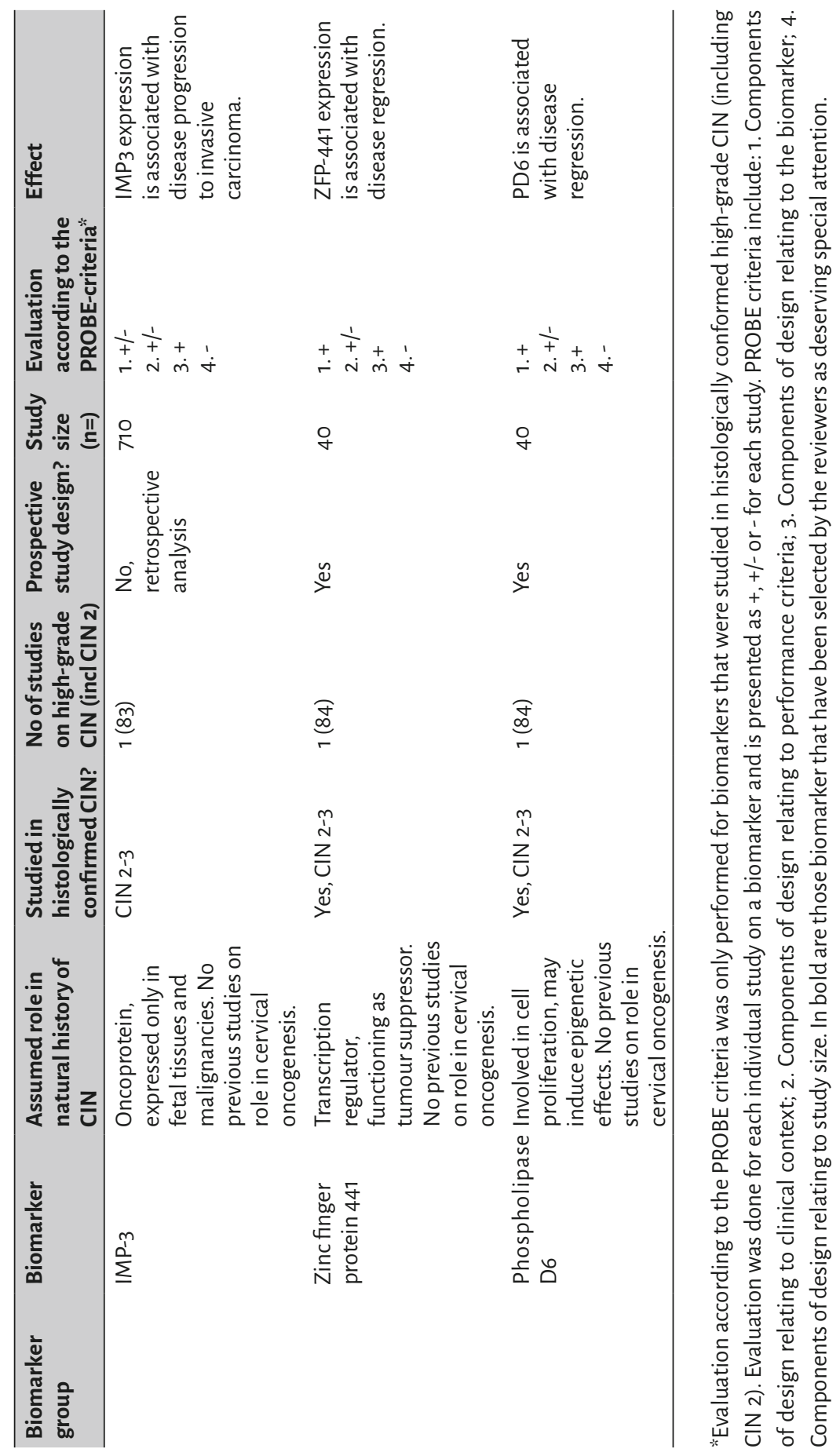


Although study results are not always consistent, additional research on markers of the immune response as prognostic biomarkers seems appropriate. After all, HPV and HPV-induced cervical lesions are cleared by the immune system. Telomerase amplification is a well-known contributor to carcinogenesis and expression hTERC (a template in telomere elongation) is associated with $\mathrm{CIN}$ grade and with progression of low-grade $\mathrm{CIN}$. No evidence is available on its prognostic value in high-grade $\mathrm{CIN}$, but its established role in carcinogenesis makes it a promising biomarker, in need of additional research. Viral genotype could serve as a prognostic biomarker. Individual differences in oncogenic potential of different HPV-types is well known and HPV16 is associated with a decreased rate of disease regression. Viral DNA methylation of $\mathrm{CpG}$-sites has recently been proven an effective diagnostic biomarker. DNA methylation, as an epigenetic effect, causes altered gene expression and has been associated with many types of cancer. Evidence on the prognostic effects of viral DNA methylation in the natural history of CIN is limited, as this marker was only recently introduced. A first study on low-grade $\mathrm{CIN}$ shows promising results, however. Cell cycle factors reflect the cellular processes causing cervical lesions. Lower p53 and pRb was associated with disease persistence in HSIL, probably reflecting in enhanced effect of viral $\mathrm{E} 6$ and E7 and increased oncogenic effect. Ki67 reflects cell proliferation as a result of increased E2F, and was associated with progression of CIN 1-2. Its prognostic effect could not be confirmed in highgrade $\mathrm{CIN}$. However, the amount of studies on the prognostic effect of Ki67 is limited and the only study on its role in high-grade CIN was performed in a small patient population. Furthermore, its prognostic value in low-grade CIN was substantial and Ki67 serves has an established role in cervical carcinogenesis. We therefore feel that further research in high-grade $\mathrm{CIN}$ seems sensible.

The above mentioned promising biomarkers have all been associated with the natural history of $\mathrm{CIN}$. However, we also advocate further investigation of HPV-induced epigenetic effects. Recently, HPV-induced methylation of host-DNA has been proven a valuable biomarker in CIN diagnostics, as was discussed before. Following on this, its value as a prognostic biomarker should be assessed.

\section{Expert commentary}

High-grade $\mathrm{CIN}$ is the precursor of cervical carcinoma, one of the most common types of cancers in women worldwide. Prevention of cervical cancer is based on both screening for cervical cancer precursor lesions and adequate treatment of such lesions. Both cervical cancer screening and treatment of high-grade $\mathrm{CIN}$ has led to a decreased incidence of cervical cancer, but screening and treatment practices can still be improved. Cervical cancer screening has recently been improved by the introduction of primary HPV-typing. Furthermore, the implementation of biomarkers in cervical cancer screening is being investigated and shows promising results. Treatment of cervical cancer precursor lesions could also be further improved, with the main purpose of reducing overtreatment and under-treatment. Because the natural behaviour of high-grade CIN is still unpredictable, most of such lesions are treated by surgical excision. Approximately $20-40 \%$ of all high-grade $\mathrm{CIN}$ lesions shows spontaneous regression. Identification of these lesions would make watchful waiting an option in selected patients, especially in those with a future pregnancy wish. Current histopathological assessment is unable to differentiate between high-grade CIN lesions that will regress spontaneously or not. Prognostic biomarkers may be helpful this differentiation. 
This review aims to identify potential prognostic biomarkers that predict the natural behaviour of high-grade $\mathrm{CIN}$. The natural history of high-grade $\mathrm{CIN}$ is determined by an interaction of viral and host factors that influence cellular processes in cervical oncogenesis. Candidate biomarkers should therefore ideally have a clear role in the natural history of CIN, reflecting associated host, viral and cellular factors. An adequate immune response seems to be of utmost importance in cervical carcinogenesis, as this should clear HPV infection or cervical precursor lesions before irreversible effects of HPV E6 and E7 on the cell cycle processes have been induced and the immune system is evaded. This "immunoediting hypothesis" was recently illustrated by Umar, and assumes sculpting of the tumours' ability to be recognized by the immune system. This leads to active immune evasion in later stages of oncogenesis, whereas early stages are recognized and subsequently cleared by the immune system.(85) In this context, it is not surprizing that methylation assays (as a surrogate marker of the accumulation of genetic and epigenetic changes as a result of persisting effects of HPV E6 and E7 expression in high-grade $\mathrm{CIN}$ ) are highly efficient at detecting advanced high-grade CIN lesions.(21)

Several candidate prognostic biomarkers for the natural history of high-grade $\mathrm{CIN}$ were identified in this review. However, none of the studies have been conducted according to the PROBE-criteria. Furthermore, the quality of evidence for those markers that have been associated with disease prognosis is often poor: studies sizes are small and selection criteria and outcome measures show great diversity. Investigation of prognostic biomarkers in high-grade CIN should ideally be performed in prospective follow-up studies on high-grade $\mathrm{CIN}$, to evaluate lesion regression or progression in relation to a biomarker at baseline. The conduction of long-term prospective observational studies is impossible for high-grade CIN patients, due to obvious ethical reasons. One such study was performed, in which women with high-grade CIN were withheld from treatment. Over $30 \%$ of these women developed cervical cancer, compared to less than $1 \%$ of women receiving treatment.(5) For the identification of candidate prospective biomarkers, we therefore rely largely on short term prospective or retrospective observational studies, in which lesion development in high-grade $\mathrm{CIN}$ patients is observed in the time interval between diagnosis and treatment, as was done by Munk and Trimble et al. $(6,8)$ This seems to be the best alternative for the evaluation of prognostic biomarkers in high-grade CIN. However, such studies should ideally be conducted according to the PROBE-criteria in order to ensure adequate and uniform interpretation of study results.

None of the reviewed candidate prognostic biomarkers is yet approaching application in clinical practice. Nevertheless, several of the identified candidate prognostic biomarkers are of special interest, as they have a clear role in the natural history of high-grade $\mathrm{CIN}$ and have been associated with disease regression or progression in one or more studies. These include HLA polymorphisms, markers of the lymphoproliferative response (CD4+, CD8+, CD25 and CD138) and telomerase amplification as host factors, viral genotype and viral DNA methylation as viral factors and cell cycle markers p53, Rb and Ki67. Additional research is needed to evaluate HPV-induced epigenetic effects as prognostic biomarkers, as current evidence is promising but insufficient to draw conclusions. As no single biomarker offers sufficient prognostic value, we believe that prediction of the natural history of $\mathrm{CIN}$ will most probably be based on a biomarker profile, consisting of 
several markers reflecting host, viral and cellular functions in cervical oncogenesis. Of the currently available composite biomarker profiles, one was developed for the natural history of CIN 1-2. The other composite biomarker profile was developed for high-grade $\mathrm{CIN}$, but could still be improved in terms of predictive value. These two composite biomarker profiles offer a good basis for further development and could be supplemented with other potential prognostic biomarkers.

In conclusion, although several promising biomarkers have been identified for the prediction of the natural history of high-grade $\mathrm{CIN}$, no single biomarker or biomarker profile is currently approaching implementation in clinical practice. Additional clinical trials are necessary to determine their true clinical value. These trials should be carried out in prospective study designs, according to the PROBE criteria.

\section{Five-year view}

In concordance with the optimization of HPV and CIN screening and diagnosis, we expect improvement in the field of CIN treatment in the next five years. Medical decision making and treatment of disease is increasingly individualized and based upon individual wishes and characteristics. Currently, all high-gradeCIN lesions are treated by LLETZ, as their natural behaviour is unpredictable. As a substantial subset of high-grade CIN lesions regress spontaneously, standard treatment with LLETZ leads to overtreatment. This is particularly disadvantageous for patients with a future pregnancy wish, as they are at increased risk of subfertility and premature birth in subsequent pregnancies. We advocate the development of an adequate biomarker profile in the next five years, by which the behaviour of the individual high-grade CIN lesions can be predicted. This offers the opportunity for an individualized treatment strategy, in which a waitand-see policy could be applied in those cases in which regression is expected. This is beneficial for both patients, who will be subjected to less overtreatment and associated side effects, and for health care providers, who benefit from less therapeutic colposcopies and probably lower costs.

Aiming to reduce overtreatment of CIN lesions, an increasing number of studies is being conducted on conservative treatment strategies for $\mathrm{CIN}$, including treatment by therapeutic vaccination, topical imiquimod or intralesional interferons. The limited study results show that these treatments may be effective, but only in a subset of patients, making these treatment modalities unfit for application in the general patient population. In line with the development of a biomarker profile for the prediction of the natural history of $\mathrm{CIN}$, a biomarker profile may be developed predicting the response to conservative treatment modalities. This may lead to the differentiation between high-grade $\mathrm{CIN}$ patients who will and will not show spontaneous regression and patients who will and will not respond to conservative treatment, leaving only a subset of patients for treatment with LLETZ.

In order to realize these goals, more research on established and potential prognostic biomarkers is necessary. The prognostic value of several viral and host factors, such as viral genotype and markers of lymphoproliferative response, and several well-known cell cycle markers has been established in the past. Other markers have only recently been identified in HPV induced lesions 
and other diseases: markers of epigenetic effects are among the most extensively studied at the moment. No single biomarker or biomarker profile currently offers sufficient prognostic value in HPV induced lesions, rendering further research for all proposed biomarkers. Obviously, highgrade CIN cannot be left untreated for unlimited follow-up, for obvious ethical reasons. However, spontaneous regression of high-grade CIN has been established and, furthermore, several studies show that follow-up of untreated high-grade CIN during a mean of 12-16 weeks offers a safe window for studies evaluating the natural history of these lesions.(6-8) We therefore advocate further evaluation of the proposed biomarkers in prospective studies, which should be conducted according to the established (e.g. PROBE) criteria. In the course of the next five years or more, this could lead to the development of a biomarker profile with high predictive values for the natural history of high-grade $\mathrm{CIN}$ and response to conservative treatment modalities, as a basis for its individualized treatment. 


\section{References}

1. Ferlay ], Shin HR, Bray F, Forman D, Mathers C, Parkin DM. Estimates of worldwide burden of cancer in 2008: GLOBOCAN 2008. International journal of cancer Journal international du cancer. 2010;127(12):2893-917.

2. zur Hausen H. Papillomaviruses causing cancer: evasion from host-cell control in early events in carcinogenesis. Journal of the National Cancer Institute. 2000;92(9):690-8.

\section{** this review illustrates that HPV is the cause of cervical cancer.}

3. Jemal A, Simard EP, Dorell C, et al. Annual Report to the Nation on the Status of Cancer, 1975-2009, featuring the burden and trends in human papillomavirus(HPV)-associated cancers and HPV vaccination coverage levels. Journal of the National Cancer Institute. 2013;105(3):175-201.

4. Peto ], Gilham C, Deacon ], et al. Cervical HPV infection and neoplasia in a large populationbased prospective study: the Manchester cohort. British journal of cancer. 2004;91(5):942-53.

5. McCredie MR, Sharples K], Paul C, et al. Natural history of cervical neoplasia and risk of invasive cancer in women with cervical intraepithelial neoplasia 3: a retrospective cohort study. The lancet oncology. 2008;9(5):425-34.

6. MunkAC, Gudlaugsson E, Ovestad IT, etal. Interaction of epithelial biomarkers, local immune response and condom use in cervical intraepithelial neoplasia 2-3 regression. Gynecologic oncology. 2012;127(3):489-94.

7. Ovestad IT, Gudlaugsson E, Skaland I, etal. The impact of epithelial biomarkers, local immune response and human papillomavirus genotype in the regression of cervical intraepithelial neoplasia grades 2-3. Journal of clinical pathology. 2011;64(4):303-7.

8. Trimble CL, Piantadosi S, Gravitt P, et al. Spontaneous regression of high-grade cervical dysplasia: effects of human papillomavirus type and HLA phenotype. Clinical cancer research : an official journal of the American Association for Cancer Research. 2005;11(13):4717-23.

9. Grimm C, Polterauer S, Natter C, et al. Treatment of cervical intraepithelial neoplasia with topical imiquimod: a randomized controlled trial. Obstetrics and gynecology. 2012;120(1):1529.

10. Barken SS, Rebolj M, Andersen ES, Lynge E. Frequency of cervical intraepithelial neoplasia treatment in a well-screened population. International journal of cancer Journal international du cancer. 2012;130(10):2438-44.

11. Spracklen CN, Harland KK, Stegmann B], Saftlas AF. Cervical surgery forcervical intraepithelial neoplasia and prolonged time to conception of a live birth: a case-control study. BJOC : an international journal of obstetrics and gynaecology. 2013;120(8):960-5.

12. Crane JM. Pregnancy outcome after loop electrosurgical excision procedure: a systematic review. Obstetrics and gynecology. 2003;102(5 Pt 1):1058-62.

13. Kyrgiou M, Koliopoulos G, Martin-Hirsch P, Arbyn M, Prendiville W, Paraskevaidis E. Obstetric outcomes after conservative treatment for intraepithelial or early invasive cervical lesions: systematic review and meta-analysis. Lancet. 2006;367(9509):489-98.

14. Arbyn M, Kyrgiou M, Simoens C, et al. Perinatal mortality and other severe adverse pregnancy outcomes associated with treatment of cervical intraepithelial neoplasia: meta-analysis. Bmj. 2008;337:a1284. 
15. Italiano A. Prognostic or predictive? It's time to get back to definitions! Journal of clinical oncology : official journal of the American Society of Clinical Oncology. 2011;29(35):4718; author reply -9.

16. Campos NG, Burger EA, Sy S, et al. An Updated Natural History Model of Cervical Cancer: Derivation of Model Parameters. American journal of epidemiology. 2014 Sep 1; 180(5):54555.

17. Wang SS, Hildesheim A.Chapter 5: Viral and host factors in human papillomavirus persistence and progression. Journal of the National Cancer Institute Monographs. 2003(31):35-40.

18. Baak JP, Kruse A], Robboy S], Janssen EA, van Diermen B, Skaland I. Dynamic behavioural interpretation of cervical intraepithelial neoplasia with molecular biomarkers. Journal of clinical pathology. 2006;59(10):1017-28.

19. Moscicki $A B$, Schiffman $M$, Burchell $A$, et al. Updating the natural history of human papillomavirus and anogenital cancers. Vaccine. 2012;30 Suppl 5:F24-33.

20. Pepe MS, Feng Z, Janes H, Bossuyt PM, Potter JD. Pivotal evaluation of the accuracy of a biomarker used for classification or prediction: standards for study design. Journal of the National Cancer Institute. 2008;100(20):1432-8.

* this paper provides a method to determine applicability of diagnostic and prognostic candidate biomarkers

21. Steenbergen RD, Snijders P], Heideman DA, Meijer C]. Clinical implications of (epi) genetic changes in HPV-induced cervical precancerous lesions. Nature reviews Cancer. 2014;14(6):395-405.

*: this paper provides an excellent overview of current knowledge on (epi)genetic changes in $\mathrm{CIN}$

22. Lungu O, Sun XW, Felix J, Richart RM, Silverstein S, Wright TC, Jr. Relationship of human papillomavirus type to grade of cervical intraepithelial neoplasia. JAMA : the journal of the American Medical Association. 1992;267(18):2493-6.

23. Munoz N. Human papillomavirus and cancer: the epidemiological evidence. Journal of clinical virology : the official publication of the Pan American Society for Clinical Virology. 2000;19(1-2):1-5.

24. Trottier H, Franco EL. The epidemiology of genital human papillomavirus infection. Vaccine. 2006;24 Suppl 1:S1-15.

25. Syrjanen K, Hakama M, Saarikoski S, et al. Prevalence, incidence, and estimated life-time risk of cervical human papillomavirus infections in a nonselected Finnish female population. Sexually transmitted diseases. 1990;17(1):15-9.

26. Cuschieri KS, Cubie HA, Whitley MW, et al. Persistent high risk HPV infection associated with development of cervical neoplasia in a prospective population study. Journal of clinical pathology. 2005;58(9):946-50.

27. Winer RL, Hughes JP, Feng Q, et al. Early natural history of incident, type-specific human papillomavirus infections in newly sexually active young women. Cancer epidemiology, biomarkers \& prevention : a publication of the American Association for Cancer Research, cosponsored by the American Society of Preventive Oncology. 2011;20(4):699-707. 
28. Moody CA, Laimins LA. Human papillomavirus oncoproteins: pathways to transformation. Nature reviews Cancer. 2010;10(8):550-60.

* this review provides an excellent overview of HPV induced molecular mechanisms leading to cervical cancer

29. Doorbar ], Quint W, Banks L, et al. The biology and life-cycle of human papillomaviruses. Vaccine. 2012;30 Suppl 5:F55-70.

*** this review provides an excellent overview of HPV biology, life cycle and HPV oncogenic properties

30. Herfs M, Somja J, Howitt BE, et al. Unique recurrence patterns of cervical intraepithelial neoplasia after excision of the squamocolumnar junction. International journal of cancer. 2015 Mar 1;136(5): 1043-52.

31. Wentzensen N, von Knebel Doeberitz M. Biomarkers in cervical cancer screening. Disease markers. 2007;23(4):315-30.

32. Jimenez-Wences H, Peralta-Zaragoza O, Fernandez-Tilapa G. Human papilloma virus, DNA methylation and microRNA expression in cervical cancer (Review). Oncology reports. 2014;31(6):2467-76.

33. Clarke MA, Wentzensen N, Mirabello L, et al. Human papillomavirus DNA methylation as a potential biomarker for cervical cancer. Cancer epidemiology, biomarkers \& prevention : a publication of the American Association for Cancer Research, cosponsored by the American Society of Preventive Oncology. 2012;21(12):2125-37.

34. Stanley MA. Epithelial cell responses to infection with human papillomavirus. Clinical microbiology reviews. 2012;25(2):215-22.

*this review provides an excellent overview of the host immune response to HPV infection and HPV immune evasion strategies

35. Patel S, Chiplunkar S. Host immune responses to cervical cancer. Current opinion in obstetrics \& gynecology. 2009;21(1):54-9.

*this review provides an excellent overview of the host immune response to HPV infection and HPV immune evasion strategies

36. Maglennon GA, Doorbar ]. The biology of papillomavirus latency. The open virology journal. 2012;6:190-7.

37. Bais AG, Beckmann I, Lindemans ], et al. A shift to a peripheral Th2-type cytokine pattern during the carcinogenesis of cervical cancer becomes manifest in CIN III lesions. Journal of clinical pathology. 2005;58(10):1096-100.

38. Sharma A, Rajappa M, Saxena A, Sharma M. Cytokine profile in Indian women with cervical intraepithelial neoplasia and cancer cervix. International journal of gynecological cancer : official journal of the International Gynecological Cancer Society. 2007;17(4):879-85.

39. Visser ], Nijman HW, Hoogenboom BN, et al. Frequencies and role of regulatory T cells in patients with (pre)malignant cervical neoplasia. Clinical and experimental immunology. 2007;150(2):199-209.

40. Doorbar ]. Papillomavirus life cycle organization and biomarker selection. Disease markers. 2007;23(4):297-313.

41. de Freitas AC, Coimbra EC, Leitao MD. Molecular targets of HPV oncoproteins: Potential biomarkers for cervical carcinogenesis. Biochimica et biophysica acta. 2014;1845(2):91-103. 
42. Hildesheim A, Wang SS. Host and viral genetics and risk of cervical cancer: a review. Virus research. 2002;89(2):229-40.

43. Matsumoto $\mathrm{K}$, Maeda $\mathrm{H}$, Oki $\mathrm{A}$, et al. HLA class II DRB1"1302 allele protects against progression to cervical intraepithelial neoplasia grade 3: a multicenter prospective cohort study. International journal of gynecological cancer : official journal of the International Gynecological Cancer Society. 2012;22(3):471-8.

44. Sastre-Carau X, Cartier I, Jourdan-Da Silva N, De Cremoux P, Lepage V, Charron D. Regression of low-grade cervical intraepithelial neoplasia in patients with HLA-DRB1*13 genotype. Obstetrics and gynecology. 2004;104(4):751-5.

45. Ovestad IT, Gudlaugsson E, Skaland I, et al. Local immune response in the microenvironment of CIN2-3 with and without spontaneous regression. Modern pathology : an official journal of the United States and Canadian Academy of Pathology, Inc. 2010;23(9):1231-40.

46. Kojima S, Kawana K, Tomio K, et al. The prevalence of cervical regulatory T cells in HPV-related cervical intraepithelial neoplasia (CIN) correlates inversely with spontaneous regression of CIN. American journal of reproductive immunology. 2013;69(2):134-41.

47. Molling JW, de Gruijl TD, Glim J, et al. CD4(+)CD25hi regulatory T-cell frequency correlates with persistence of human papillomavirus type 16 and $T$ helper cell responses in patients with cervical intraepithelial neoplasia. International journal of cancer Journal international du cancer. 2007;121(8):1749-55.

48. Liu H, Liu S, Wang H, et al. Cenomic amplification of the human telomerase gene (hTERC) associated with human papillomavirus is related to the progression of uterine cervical dysplasia to invasive cancer. Diagnostic pathology. 2012;7:147.

49. Lan YL, Yu L, Jia CW, Wu YM, Wang SY. Gain of human telomerase RNA gene is associated with progression of cervical intraepithelial neoplasia grade I or II. Chinese medical journal. 2012;125(9):1599-602.

50. Chay DB, Cho H, Kim BW, Kang ES, Song E, Kim JH. Clinical significance of serum anti-human papillomavirus 16 and 18 antibodies in cervical neoplasia. Obstetrics and gynecology. 2013;121(2 Pt 1):321-9.

51. Matsumoto K, Yoshikawa H, Yasugi T, et al. Balance of IgC subclasses toward human papillomavirus type 16 (HPV16) L1-capsids is a possible predictor for the regression of HPV16-positive cervical intraepithelial neoplasia. Biochemical and biophysical research communications. 1999;258(1):128-31.

52. de Gruijl TD, Bontkes H], Walboomers JM, et al. Immunoglobulin G responses against human papillomavirus type 16 virus-like particles in a prospective nonintervention cohort study of women with cervical intraepithelial neoplasia. Journal of the National Cancer Institute. 1997;89(9):630-8.

53. Matsumoto K, Yasugi T, Oki A, et al. IgC antibodies to HPV16, 52, 58 and 6 L1-capsids and spontaneous regression of cervical intraepithelial neoplasia. Cancer letters. 2006;231(2):30913.

54. Wilting SM, Snijders P], Verlaat $W$, et al. Altered microRNA expression associated with chromosomal changes contributes to cervical carcinogenesis. Oncogene. 2013;32(1):106-16.

55. Zheng ZM, Wang X. Regulation of cellular miRNA expression by human papillomaviruses. Biochimica et biophysica acta. 2011;1809(11-12):668-77. 
56. Wentzensen N, Sherman ME, Schiffman M, Wang SS. Utility of methylation markers in cervical cancer early detection: appraisal of the state-of-the-science. Cynecologic oncology. 2009;112(2):293-9.

57. Verhoef VM, Bosgraaf RP, van Kemenade F], et al. Triage by methylation-marker testing versus cytology in women who test HPV-positive on self-collected cervicovaginal specimens (PROHTECT-3): a randomised controlled non-inferiority trial. The lancet oncology. 2014;15(3):315-22.

58. De Strooper LM, Hesselink BA, Berkhof ], et al. Combined CADM1/MAL methylation and cytology testing forcolposcopy triage of high-riskHPV-positive women.Cancer epidemiology, biomarkers \& prevention : a publication of the American Association for Cancer Research, cosponsored by the American Society of Preventive Oncology. 2014 Sep;23(9):1933-7.

59. Kataja V, Syrjanen K, Syrjanen S, et al. Prospective follow-up of genital HPV infections: survival analysis of the HPV typing data. European journal of epidemiology. 1990;6(1):9-14.

60. Syrjanen K, Parkkinen S, Mantyjarvi R, et al. Human papillomavirus (HPV) type as an important determinant of the natural history of HPV infections in uterine cervix. European journal of epidemiology. 1985;1(3):180-7.

61. Syrjanen K, Mantyjarvi R, Vayrynen M, et al. Human papillomavirus (HPV) infections involved in the neoplastic process of the uterine cervix as established by prospective followup of 513 women for two years. European journal of gynaecological oncology. 1987;8(1):5-16.

62. Matsumoto K, Oki A, Furuta R, et al. Predicting the progression of cervical precursor lesions by human papillomavirus genotyping: a prospective cohort study. International journal of cancer Journal international du cancer. 2011;128(12):2898-910.

63. Mirabello L, Schiffman M, Ghosh A, et al. Elevated methylation of HPV16 DNA is associated with the development of high grade cervical intraepithelial neoplasia. International journal of cancer Journal international du cancer. 2013;132(6):1412-22.

64. Oka N, Kajita M, Nishimura R, Ohbayashi C, Sudo T. L1 gene methylation in high-risk human papillomaviruses for the prognosis of cervical intraepithelial neoplasia. International journal of gynecological cancer : official journal of the International Gynecological Cancer Society. 2013;23(2):235-43.

65. Sarmadi S, Izadi-mood N, Pourlashkari M, Yarandi F, Sanii S. HPV L1 capsid protein expression in squamous intraepithelial lesions of cervix uteri and its relevance to disease outcome. Archives of gynecology and obstetrics. 2012;285(3):779-84.

66. Izadi-Mood N, Sarmadi S, Eftekhar Z, Jahanteegh HA, Sanii S. Immunohistochemical expression of p16 and HPV L1 capsid proteins as predictive markers in cervical lesions. Archives of gynecology and obstetrics. 2014 Jun;289(6):1287-92.

67. Choi YS, Kang WD, Kim SM, et al. Human papillomavirus L1 capsid protein and human papillomavirus type 16 as prognostic markers in cervical intraepithelial neoplasia 1. International journal of gynecological cancer : official journal of the International Gynecological Cancer Society. 2010;20(2):288-93.

68. Rauber D, Mehlhorn G, Fasching PA, Beckmann MW, Ackermann S. Prognostic significance of the detection of human papilloma virus L1 protein in smears of mild to moderate cervical intraepithelial lesions. European journal of obstetrics, gynecology, and reproductive biology. 2008;140(2):258-62. 
69. Wang SM, Colombara D, Shi JF, et al. Six-year regression and progression of cervical lesions of different human papillomavirus viral loads in varied histological diagnoses. International journal of gynecological cancer : official journal of the International Gynecological Cancer Society. 2013;23(4):716-23.

70. Kim JW, Song SH, Jin CH, Lee JK, Lee NW, Lee KW. Factors affecting the clearance of high-risk human papillomavirus infection and the progression of cervical intraepithelial neoplasia. The Journal of international medical research. 2012;40(2):486-96.

71. Kadish AS, Timmins P, Wang Y, et al. Regression of cervical intraepithelial neoplasia and loss of human papillomavirus (HPV) infection is associated with cell-mediated immune responses to an HPV type 16 E7 peptide. Cancer epidemiology, biomarkers \& prevention : a publication of the American Association for Cancer Research, cosponsored by the American Society of Preventive Oncology. 2002;11(5):483-8.

72. Peng S, Trimble C, Wu L, et al. HLA-DQB1"02-restricted HPV16 E7 peptide-specific CD4+ T-cell immune responses correlate with regression of HPV16-associated high-grade squamous intraepithelial lesions. Clinical cancer research : an official journal of the American Association for Cancer Research. 2007;13(8):2479-87.

73. Manawapat A, Stubenrauch F, Russ R, Munk C, Kjaer SK, Iftner T. Physical state and viral load as predictive biomarkersfor persistence and progression of HPV16-positive cervical lesions: results from a population based long-term prospective cohort study. American journal of cancer research. 2012;2(2):192-203.

74. Li W, Wang W, Si M, et al. The physical state of HPV16 infection and its clinical significance in cancer precursor lesion and cervical carcinoma. Journal of cancer research and clinical oncology. 2008;134(12):1355-61.

75. Tornesello ML, Buonaguro L, Giorgi-Rossi P, Buonaguro FM. Viral and cellular biomarkers in the diagnosis of cervical intraepithelial neoplasia and cancer. BioMed research international. 2013;2013:519619.

*: this review provides an overview of current knowledge on validated and candidate biomarkers, mostly diagnostic

76. Kruse A], Skaland I, Janssen EA, et al. Quantitative molecular parameters to identify low-risk and high-risk early CIN lesions: role of markers of proliferative activity and differentiation and $\mathrm{Rb}$ availability. International journal of gynecological pathology : official journal of the International Society of Cynecological Pathologists. 2004;23(2):100-9.

77. Quint KD, de Koning MN, Quint WG, Pirog EC. Progression of cervical low grade squamous intraepithelial lesions: in search of prognostic biomarkers. European journal of obstetrics, gynecology, and reproductive biology. 2013;170(2):501-6.

78. Baak JP, Kruse AJ, Garland SM, et al. Combined p53 and retinoblastoma protein detection identifies persistent and regressive cervical high-grade squamous intraepithelial lesions. The American journal of surgical pathology. 2005;29(8):1062-6.

79. Kruse A], Baak JP, Janssen EA, et al. Low- and high-risk CIN 1 and 2 lesions: prospective predictive value of grade, HPV, and Ki67 immuno-quantitative variables. The Journal of pathology. 2003;199(4):462-70. 
80. Darragh TM, Colgan T], Thomas Cox ], et al. The Lower Anogenital Squamous Terminology Standardization project for HPV-associated lesions: background and consensus recommendations from the College of American Pathologists and the American Society for Colposcopy and Cervical Pathology. International journal of gynecological pathology : official journal of the International Society of Cynecological Pathologists. 2013;32(1):76-115.

81. Tsoumpou I, Arbyn M, Kyrgiou M, et al. p16(INK4a) immunostaining in cytological and histological specimens from the uterine cervix: a systematic review and meta-analysis. Cancer treatment reviews. 2009;35(3):210-20.

82. Omori M, Hashi A, Nakazawa K, et al. Estimation of prognoses for cervical intraepithelial neoplasia 2 by p16INK4a immunoexpression and high-risk HPV in situ hybridization signal types. American journal of clinical pathology. 2007;128(2):208-17.

83. Lu D, Yang $X$, Jiang NY, et al. IMP3, a new biomarker to predict progression of cervical intraepithelial neoplasia into invasive cancer. The American journal of surgical pathology. 2011;35(11):1638-45.

84. Uleberg KE, Ovestad IT, Munk AC, et al. Prediction of spontaneous regression of cervical intraepithelial neoplasia lesions grades 2 and 3 by proteomic analysis. International journal of proteomics. 2014;2014:129064.

85. Umar A. Cancer immunoprevention: a new approach to intercept cancer early. Cancer prevention research. 2014;7(11):1067-71.

86. Litjens R], Hopman AH, van de Vijver KK, Ramaekers FC, Kruitwagen RF, Kruse A]. Molecular biomarkers in cervical cancer diagnosis: a critical appraisal. Expert opinion on medical diagnostics. 2013;7(4):365-77.(86) 



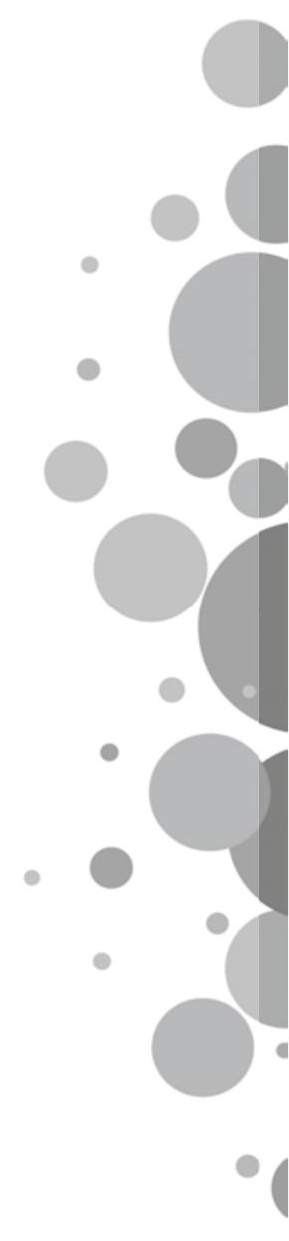




\section{CHAPTER 3}

\section{A common epitope in HLA-DRB1"13/14}

may protect against HPV16 related high-grade cervical intraepithelial neoplasia

Margot M Koeneman, Irene T Ovestad, Jennifer Schellekens, Ane Cecilie Munk, Emiel AM Janssen, Marcel G] Tilanus, Roy FPM Kruitwagen, Arnold ] Kruse

Submitted 


\section{Abstract}

Objective: Several Human Leucocyte Antigen (HLA) alleles have been associated with a decreased risk of Cervical Intraepithelial Neoplasia (CIN) and its natural prognosis. This study aims to assess HLA-A, -B, -C, -DRB1 and -DQB1 alleles as prognostic biomarkers in high-grade CIN.

Methods: 34 patients with high-grade $\mathrm{CIN}$ were selected from a previous prospective population based cohort study, conducted at the Stavanger University Hospital, Norway. Patients were followed for a median of 112 days follow-up, from biopsy to loop excision. HLA-A, -B, -C, -DRB1 and $-D Q B 1$ typing was performed in biobank stored blood samples. The influence of HLA alleles on spontaneous regression and HPV16 status of high-grade CIN was assessed by use of contingency tables, using Fisher's exact test to determine statistical significance $(p \leq 0.05)$.

Results: HLA typing analysis was successful in 32 patients. 14 patients showed disease regression. No association was found between all studied HLA allele groups and spontaneous regression of high-grade CIN. A significant association was found between HLA-DRB1*13/14 alleles and HPV status: all eight patients with these alleles were HPV16-negative, while HPV16 positive lesions did occur in patients without an HLA-DRB1*13/14 allele.

Conclusion: HLA-DRB1*13/14 may be protective against HPV16-induced high-grade CIN, which indicates an early and HPV16-specific protective effect of an HLA-DRB1*13/14 epitope. 


\section{Introduction}

Cervical intraepithelial neoplasia (CIN) is caused by infection with human papilloma virus (HPV) and is considered to be the precursor of cervical carcinoma. While high-grade CIN lesions can progress to cervical carcinoma, approximately $20-40 \%$ of these lesions show spontaneous regression.[1-4] Currently, the prognosis of individual lesions remains unpredictable, but the quest for prognostic biomarkers is ongoing. Identification of biomarkers, predicting spontaneous regression of high-grade $\mathrm{CIN}$, may enable a more individualized treatment. Patients with a high likelihood for spontaneous disease regression can then be selected for conservative management by watchful waiting. A potential biomarker may be found in the Human Leucocyte Antigen (HLA) molecules. The HLA gene complex encodes the Major Histocompatibility Complex receptors on the human cell-surface that enable antigen presentation to host immune cells. Clearance of CIN lesions is an immune-mediated process, which is largely effected by a cellular immune reaction. [5] As such, differences in HLA repertoire could influence the individual capacity for spontaneous regression of the $\mathrm{CIN}$ lesion.

Epidemiological studies indeed show that differences in HLA repertoire influence the individual susceptibility for the development of CIN lesions and cervical carcinoma.[6-8] Moreover, several $\mathrm{HLA}$ alleles have been associated with regression or persistence of CIN lesions, which is the focus of the current study. $[4,9,10]$ HLA-DRB1*13 has been associated with spontaneous regression and non-progression of low-grade CIN lesions, independently of HPV genotype.[9, 10] Only one publication studied the influence of HLA alleles on the spontaneous regression of high-grade $\mathrm{CIN}$ lesions and found that HLA-A*02:01 is associated with persistence of high-grade $\mathrm{CIN}$, but only in non-HPV 16 positive lesions. [4] The influence of HLA-DRB1*13 on spontaneous regression in highgrade $\mathrm{CIN}$ lesions was not assessed in this study.

In summary, there is some evidence that HLA repertoire may influence spontaneous regression of high-grade CIN lesions. However, not all HLA genes have been studied in regard to spontaneous regression of these lesions and it remains unclear whether the protective effect of different HLA alleles on the development of cervical lesions is HPV specific.

We hypothesize that HLA alleles could function as prognostic biomarkers in high-grade CIN. The goal of our study is to assess the influence of HLA-A, HLA-B, HLA-C, HLA-DRB1 and HLA-DQB1 on the spontaneous regression of high-grade CIN lesions. We also analyzed the association between these HLA alleles and the prevalence of HPV genotypes in high-grade CIN lesions, to assess a potential HPV-specific protective effect.

\section{Materials and methods}

The study has been performed and reported according to the STRECA guidelines for the reporting of genetic association studies and according to the PROBE criteria for biomarker research.[11, 12] 


\section{Study design and patient population}

The patient population was extracted from a prospective population based cohortstudy, conducted at the Stavanger University Hospital, Norway.[2] The original cohort consisted of 162 women aged 25-41 years, with histologically proven $\mathrm{CIN} 2$ or $\mathrm{CIN} 3$ lesions. The lesions were diagnosed in diagnostic biopsies at baseline. All women underwent a Loop Electrosurgical Excision Procedure (LEEP) after a median of 113 days follow-up (range 84-171 days). The natural history of the baseline cervical lesion during the follow-up period was evaluated in the LEEP specimen. Regression was defined as $\mathrm{CIN} 1$ or less in the LEEP specimen. Histological evaluation was performed on standard Hematoxylin Erythrosin Saffran (HES) sections, according to the World Health Organization criteria. Ki67 and p16 were used to support their diagnoses. HPV genotyping was performed by Linear Array (Roche) on DNA- material from the formalin fixed paraffin embedded cervical biopsy at inclusion. Details on histological evaluation and HPV genotyping can be found in the original article.[5] From this cohort, 34 patients were included for analysis in the current study, based on the availability of sufficient blood samples for HLA analysis. EDTA blood was sampled at the same time as the biopsy and stored in a biobank.

\section{DNA isolation}

DNA isolation was performed by qualified staff with expertise in molecular biology in a Molecular Laboratory at the Pathology Department of Stavanger University Hospital, Stavanger, Norway. Until use the DNA had been stored in Biosphere safe seal Eppendorf tubes, (Sarstedt Inc., Thermo Fisher Scientific, MA, US) in a $-80^{\circ} \mathrm{C}$ biobank freezer. The EZNA ${ }^{\circ}$ Tissue kit (Omega biotek, Norcross CA, USA) was used for purification of DNA from $250 \mu$ l frozen whole blood diluted $(4.429 \mathrm{mmol} / \mathrm{l})$ with the anticoagulant Ethylene Diamine Tetraacetic Acid (EDTA). The protocol for Blood and body fluids was followed according to the manufacturer's instructions. DNA was purified using the HiBind ${ }^{\circ}$ DNA Mini Column and eluted in $50 \mu$ l Elution Buffer.

\section{HLA procedure}

The HLA procedure was performed at the Tissue Typing Laboratory of the Department of Transplantation Immunology, Maastricht University Medical Centre, Maastricht, The Netherlands. The tissue typing laboratory has been accredited by EFI. HLA class I and class II typing was performed using the Luminex (Austin, TX, USA) sequence specific oligonucleotide (SSO) hybridization method. The SSO kits (One Lambda, Los Angeles, CA, USA) were used according to the manufacturer's guidelines.[13] DNA amplification and hybridization were automated using the LabXpress (One Lambda). The fluorescence values were read by a Luminex Labscan and HLA typing assignments were obtained using HLA Fusion 3.0 software (One Lambda). All data have been generated simultaneously (i.e. using the same reagents, conditions and allele assignment databases (IPD-IMCT/HLA)). Blinding was not applicable, as HLA typing is not subject to interpretation.

\section{Outcome measures and criteria for biomarker performance}

The outcome measures were defined as (1) the correlation between HLA alleles and spontaneous regression of high-grade CIN and (2) the correlation between HLA alleles and HPV genotype in high-grade $\mathrm{CIN}$ lesions. No previous biomarker performance values are available for HLA types. 
The required test performance values include a high specificity and positive predictive value: lesions that will not regress spontaneously must be identified, as treatment is necessary in these women. The actual values depend on the follow-up term of observational management. Lower values can be accepted when strict histological follow-up is implemented to identify persisting lesions at an early stage.

\section{Statistical analysis}

Statistical analyses were performed using Graphpad Prism version 5.01 (Graphpad Software, 2007, La Jolla California, CA, USA). The correlation between HLA alleles (HLA-A, -B, -C, -DRB1 and $-\mathrm{DQB1}$ ) and spontaneous regression or HPV-subtype were analyzed by contingency tables, using Fisher's exact test to determine statistical significance $(p \leq 0.05)$. The Hardy-Weinberg equilibrium was not considered, as the focus of our study was not to calculate allele frequencies of the HLA alleles. We have assumed that the typed alleles are expressed on the host cell surfaces. Sample size calculation was not feasible, due to the lack of comparable biomarker performance values and limitation of the study population by the availability of material.

\section{Nomenclature}

The nomenclature for defining HLA alleles has been based on the IPD-HLA/IMGT allele database that has been established according the WHO nomenclature committee.

\section{Results}

\section{Patient population}

34 patients were included in the study. Two patients were excluded from the analysis, as all HLA typing reactions failed and no further DNA was available. Patient characteristics of the 32 remaining patients can be found in table 1 . There was no missing data regarding the patient characteristics. Between patients with and without disease regression, there were no statistical differences mean age (30.6 vs 30.3 years, $p=0.86$ ), biopsy-LEEP interval ( 110 vs 114 days, $p=0.29$ ) and HPV16 positivity (5/14 (36\%) vs 7/18 (39\%), $p=0.86)$.

\section{Table 1. Patient characteristics}

\begin{tabular}{ll}
\hline Characteristic & Outcome $\mathbf{N}=\mathbf{3 2}$ \\
\hline Age (mean, range in years) & $30.5(25-40)$ \\
Biopsy-LEEP interval (mean, range in days) & $112(84-149)$ \\
HPV 16 positive (N, \%) & $12(38 \%)$ \\
Spontaneous disease regression overall (N, \%) & $14(44 \%)$ \\
\hline
\end{tabular}

\section{HLA repertoire and spontaneous regression of high-grade CIN}

The results of the HLA-typing reactions can be found in table 2. In five patients, one or more HLA typing reactions failed; no further DNA was available for additional typing. No significant associations were found between the studied HLA genes and spontaneous regression of highgrade $\mathrm{CIN}$ in the entire study population, nor in the subgroups of HPV16 positive lesions and HPV16 negative lesions. 


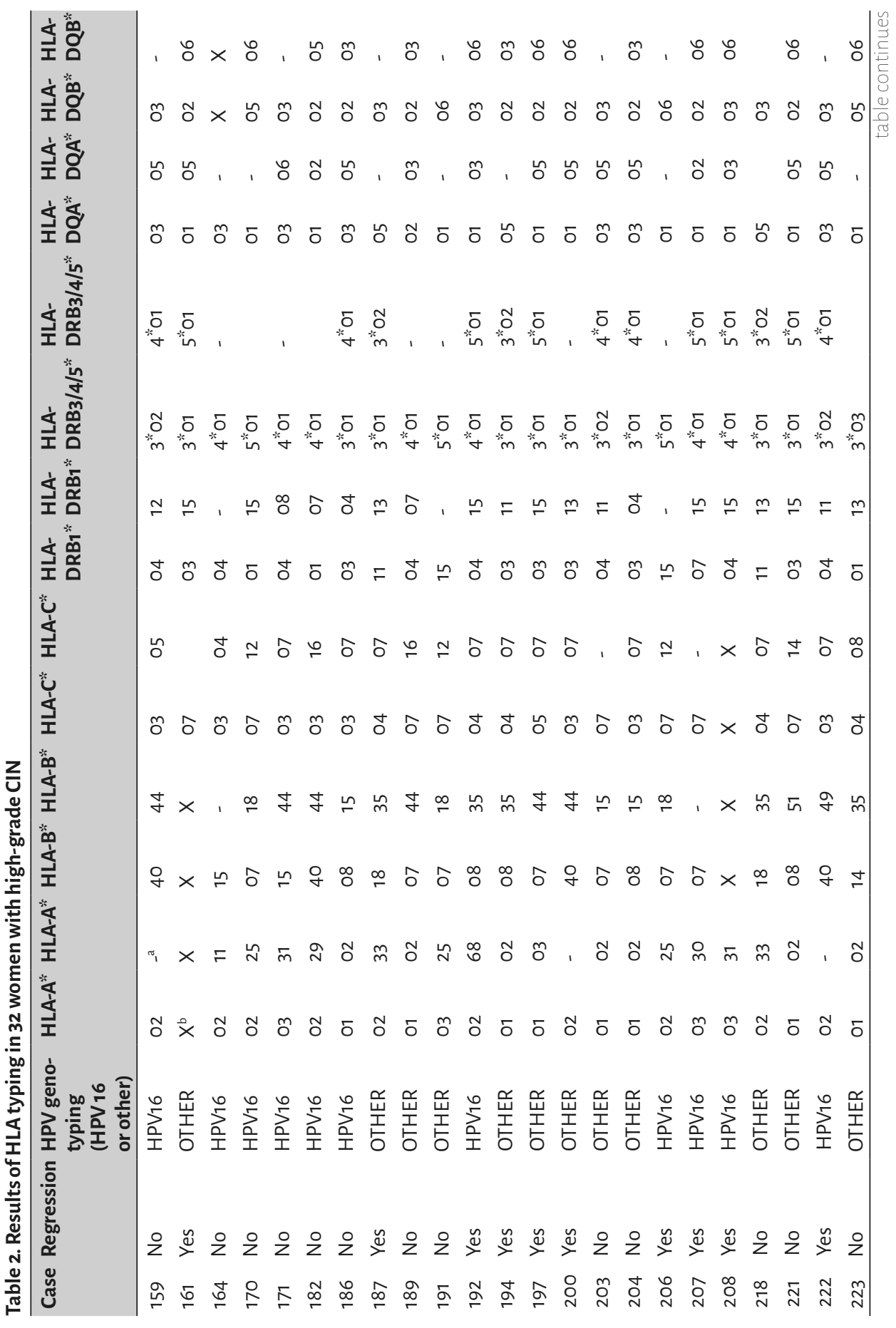




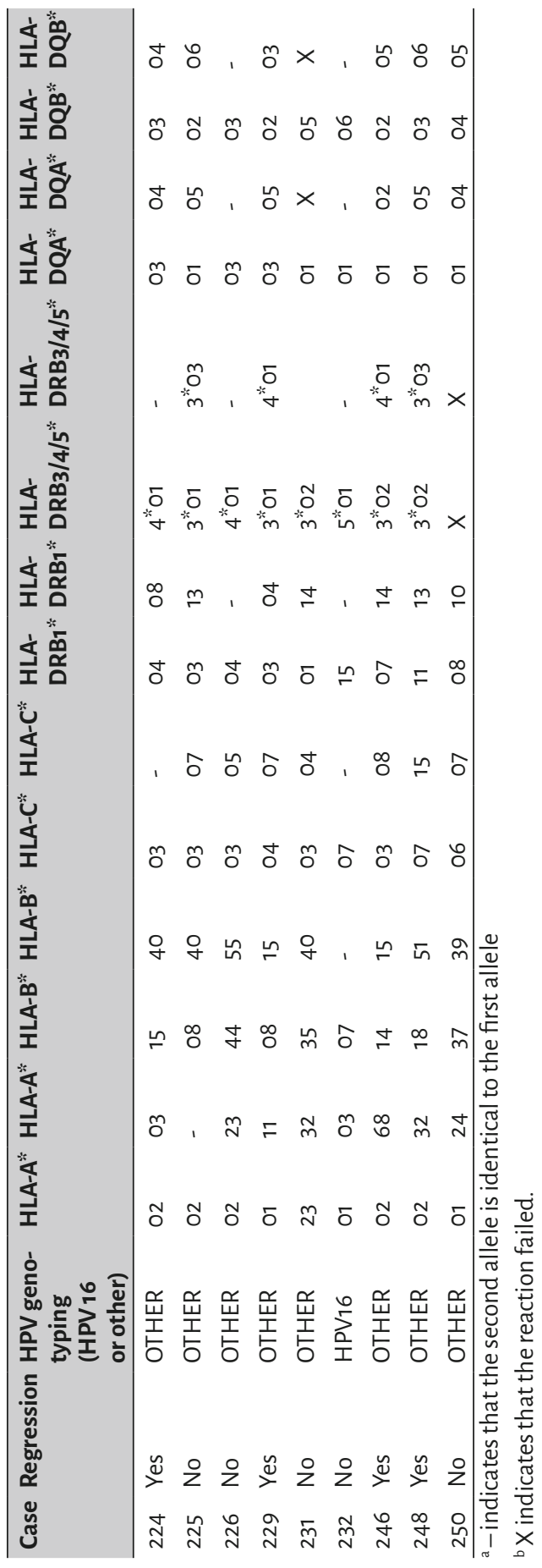




\section{HLA repertoire and HPV status}

A significant association was found between HLA-DRB1"13/14 alleles and HPV status. The lesions of all eight patients with an HLA-DRB1*13/14 allele were HPV16-negative, while the lesions of patients without an HLA-DRB1"13/14 allele were equally frequent HPV16 positive $(n=12)$ as HPV16 negative $(n=12)(p=0.0135)$. Results are presented in table 3 . There was a trend towards HPV16 negativity in patients with an HLA-DRB1"13 allele: all six patients with an HLA-DRB1*13 allele were HPV16-negative. Of those patients without an HLA-DRB1"13 allele, 12 were HPV16 positive and 14 were HPV16 negative $(p=0.0613)$. No other significant associations were found between other HLA alleles and HPV status.

Table 3. HLA-DRB1*13/14 in relation to HPV status in high-grade CIN lesions

\begin{tabular}{lllllll}
\hline & DRB1*13 & No DRB1*13 & DRB1*14 & No DRB1*14 & DRB1*13/14 & No DRB1*13/14 \\
\hline HPV 16 & 0 & 12 & 0 & 12 & 0 & 12 \\
Others & 6 & 14 & 2 & 18 & 8 & 12 \\
& $\mathrm{p}=0.0613$ & & $\mathrm{p}=0.5161$ & & $\mathrm{p}=0.0135$ & \\
\hline
\end{tabular}

\section{Discussion}

The results of this study show a potential protective effect of the HLA-DRB1"13/14 allele group against $\mathrm{HPV}_{16} 6$ positive $\mathrm{CIN}$, suggesting a common epitope in the DRB1*13/14 allele group. None of the women with an HLA-DRB1*13/14 allele group had an HPV16 positive CIN lesion. In our cohort, no correlation of any HLA-A, -B, -C, -DRB1 or -DQB1 alleles with spontaneous regression of high-grade CIN was observed.

Previous publications found significant influences of certain HLA types on the spontaneous regression of both low- and high-grade CIN lesions. In low-grade lesions, HLA-DRB1"13 has been associated with spontaneous regression and HLA-DRB1*13:02 was found to protect against progression to $\operatorname{CIN} 3 .[9,10,14]$ Only one study has described the influence of the HLA repertoire on the spontaneous regression of high-grade $\mathrm{CIN}$ lesions. In this study, women with an HLA-A*02:01 allele were significantly less likely to clear a $\mathrm{CIN}$ 2/3 lesion than women without this allele ( $14.3 \%$ vs $42.3 \%, p=0.05$ ), but only in HPV16 negative lesions. [4] The influence of HLA$\mathrm{DRB}$ "13 on spontaneous regression in high-grade CIN lesions was not assessed in this study. Our study did not show a protective effect of HLA-DRB1"13 in high-grade CIN lesions. Neither could we confirm the previously established association between the HLA-A*02:01 allele and the natural history of high-grade CIN lesions. This could be caused by the limited number of patients in our study. However, the lack of a protective effect of HLA-DRB1*13 in high-grade CIN lesions represented in our cohort may also be explained by the possibility that the protective effect of the HLA-DRB1"13 allele against cervical carcinoma occurs in the early stages of CIN, and not in the stage of established CIN3 lesions or lesions progressing to cervical cancer. Rather, the HLA$\mathrm{DRB}_{1}{ }^{* 13}$ allele may be protective against the development of high-grade $\mathrm{CIN}$ in the first place. This theory is supported by population frequency studies, showing a similar frequency of the HLA-DRB1"13 allele in patients with low-grade lesions as compared to the general population, 
but a reduced frequency in patients with high-grade $\mathrm{CIN}$ and cervical carcinoma. $[6,7,9,14-17]$ A possible mechanism for this theory may be that the protective influence of the HLA-DRB1 13 allele is larger when the expression of viral oncogenes is limited, which is the case in low-grade $\mathrm{CIN}$ lesions. Indeed, spontaneous regression of these lesions is more common in women with the HLA-DRB1" 13 allele. Theoretically, this could prevent a persistent infection, thereby preventing a transforming infection leading to high-grade $\mathrm{CIN}$ lesions. Once a high-grade $\mathrm{CIN}$ lesion has developed, the protective effect of HLA-DRB1" 13 is most likely counterbalanced by the increased expression of viral oncogenes, which is reflected in the fact that the HLA-DRB1"13 allele does not seem to increase spontaneous regression of high-grade CIN. Based on these findings, we postulate that the protective effect of HLA-DRB1" 13 may be exerted mainly through a decreased susceptibility to high-grade CIN lesions, rather than through the promotion of spontaneous regression of these lesions. This may explain why an effect on the natural prognosis of high-grade lesions was not found in this study.

Our study indicates an HPV16 specific protective effect of the HLA-DRB1"13/14 allele group. None of the patients with an HLA-DRB1"13/14 allele had an HPV16 positive CIN lesion. This may imply that a specific HLA-DRB1*13/14 epitope is selectively protective against HPV16-induced CIN. A biological explanation for this could be that protective HLA molecules bind HPV16 with greater affinity than non-protective HLA molecules. Indeed, a study on HPV peptide sequences bound to several HLA-DR and-DQ molecules revealed that HPV16 peptide epitopes bind with higher affinity to the protective than to non-protective HLA-DR and-DQ molecules. [18] This may lead to a more effective immune response. Since HPV16 is more likely than other HPV types to induce high-grade CIN lesions, this could also explain the protective effect of HLA-DRB1*13 against the development of high-grade $\mathrm{CIN}$ lesions. However, previous studies have shown conflicting results regarding the association between HLA-DRB1*13/14 and HPV16. Several authors found an HPV16 dependent association between the HLA-DRB1*13 and HLA-DRB1"14 alleles and cervical carcinoma. $[8,19]$ Other authors, however, have not confirmed this finding.[9, 17, 20, 21] Eiguchi et al. performed the only study in patients with high-grade CIN (in combination with cervical carcinoma patients) and found a significant negative association between HPV16 and HLA-DRB1*13.[15] This is in agreement with our finding that HLA-DRB1*13 seems to protect against HPV16 induced highgrade $\mathrm{CIN}$. In our hypothesis that HLA-DRB1"13 has a protective effect against the development of high-grade $\mathrm{CIN}$, this could imply that this protective effect is caused preferentially through an increased immune response to HPV16. Further studies on HPV16 peptide epitopes and HLA$\mathrm{DRB}^{*} 13$ in both low- and high-grade CIN should clarify this issue.

Our study is the first to assess the associations between HLA-DRB1"13/14 alleles and the natural history and HPV16 status in high-grade $\mathrm{CIN}$, but several limitations must be addressed. A major limitation of the current study is the limited sample size. From the historical cohort of patients, sufficient material was available for HLA typing in 34 patients only. As the HLA complex is highly polymorphic, small patient populations may cause difficulties establishing significant results. Another limitation is that linkage disequilibrium was not assessed in this study. HLA-DRB 1 " 13 has been shown to be in linkage disequilibrium with $D Q B 1^{*} 06 .[6,14]$ Previous studies have not clarified whether the protective effect is caused by either the individual alleles or by the combination of 
alleles. [8, 20] Further research in larger populations should focus on this issue. A final limitation may be that we did not specify the HLA-DRB1"13 allele further. Whereas Sastre-Garau et al. studied the correlation of the HLA-DRB1"13 allele and spontaneous regression, Matsumoto et al. focused on the HLA-DRB1"13:02 allele. $[9,10]$ Other studies show that the protective effect of HLADRB1"13 appeared to be contributed by individual alleles: HLA-DRB1*13:01, "13:02 and *13:03. [7, 19] Studying the overall HLA-DRB1"13 allele group may therefore mask the protective effects of individual alleles. The limited availability of DNA material in our study, however, did not facilitate further specification of individual alleles.

In conclusion, we found no effect of the HLA repertoire on spontaneous regression of high-grade $\mathrm{CIN}$ in the current patient group and could therefore not identify HLA alleles as a prognostic biomarker in high-grade CIN. We did find a protective effect of the HLA-DRB1*13/14 allele group against HPV16 induced high-grade CIN. Given the established fact that HLA-DRB1"13 is protective against $\mathrm{CIN}$ and cervical cancer and has been shown to induce spontaneous regression of lowgrade $\mathrm{CIN}$, we hypothesize that the protective effect of HLA-DRB1*13 is exerted by preventing the development of high-grade lesions, rather than promoting spontaneous regression of these lesions. This protective effect may be exerted through an enhanced immune response to HPV16. Further research is necessary to test our hypothesis and to establish the mode of action of this protective effect. 


\section{References}

1. Grimm C, Polterauer S, Natter C, Rahhal ], Hefler L, Tempfer CB, et al. Treatment of cervical intraepithelial neoplasia with topical imiquimod: a randomized controlled trial. Obstet Gynecol. 2012;120(1):152-9.

2. Munk AC, Gudlaugsson E, Ovestad IT, Lovslett K, Fiane B, Hidle B, et al. Interaction of epithelial biomarkers, local immune response and condom use in cervical intraepithelial neoplasia 2-3 regression. Gynecol Oncol. 2012;127(3):489-94.

3. Ovestad IT, Gudlaugsson E, Skaland I, Malpica A, Munk AC, Janssen EA, et al. The impact of epithelial biomarkers, local immune response and human papillomavirus genotype in the regression of cervical intraepithelial neoplasia grades 2-3. J Clin Pathol. 2011;64(4):303-7.

4. Trimble CL, Piantadosi S, Gravitt P, Ronnett B, Pizer E, Elko A, et al. Spontaneous regression of high-grade cervical dysplasia: effects of human papillomavirus type and HLA phenotype. Clin Cancer Res. 2005;11(13):4717-23.

5. Stanley MA. Epithelial cell responses to infection with human papillomavirus. Clin Microbiol Rev. 2012;25(2):215-22.

6. Hildesheim A, Wang SS. Host and viral genetics and risk of cervical cancer: a review. Virus Res. 2002;89(2):229-40.

7. Zoodsma M, Nolte IM, Te Meerman G], De Vries EG, Van der Zee AG. HLA genes and other candidate genes involved in susceptibility for (pre)neoplastic cervical disease. Int ] Oncol. 2005;26(3):769-84.

8. Leo P], Madeleine MM, Wang S, Schwartz SM, Newell F, Pettersson-Kymmer U, et al. Defining the genetic susceptibility to cervical neoplasia-A genome-wide association study. PLoS Genet. 2017;13(8):e1006866.

9. Sastre-Carau X, Cartier I, Jourdan-Da Silva N, De Cremoux P, Lepage V, Charron D. Regression of low-grade cervical intraepithelial neoplasia in patients with HLA-DRB1*13 genotype. Obstet Gynecol. 2004;104(4):751-5.

10. Matsumoto K, Maeda H, Oki A, Takatsuka N, Yasugi T, Furuta R, et al. HLA class II DRB1*1302 allele protects against progression to cervical intraepithelial neoplasia grade 3: a multicenter prospective cohort study. Int ] Gynecol Cancer. 2012;22(3):471-8.

11. Little J, Higgins JP, loannidis JP, Moher D, Gagnon F, von Elm E, et al. Strengthening the reporting of genetic association studies (STRECA): an extension of the strengthening the reporting of observational studies in epidemiology (STROBE) statement. ] Clin Epidemiol. 2009;62(6):597-608 e4.

12. Pepe MS, Feng Z, Janes H, Bossuyt PM, Potter JD. Pivotal evaluation of the accuracy of a biomarker used for classification or prediction: standards for study design. J Natl Cancer Inst. 2008;100(20):1432-8.

13. Dunbar SA. Applications of Luminex XMAP technology for rapid, high-throughput multiplexed nucleic acid detection. Clin Chim Acta. 2006;363(1-2):71-82.

14. Matsumoto K, Maeda H, Oki A, Takatsuka N, Yasugi T, Furuta R, et al. Human leukocyte antigen class II DRB1"1302 allele protects against cervical cancer: At which step of multistage carcinogenesis? Cancer Sci. 2015;106(10):1448-54. 
15. Eiguchi K, Tatti S, Alonio LV, Conzalez JV, Leiros G], Fleider L, et al. Association of DRB1 and DQB1 HLA class II polymorphisms in high-grade and neoplastic cervical lesions of women from Argentina. J Low Genit Tract Dis. 2008;12(4):262-8.

16. Heusinkveld M, Welters M], van Poelgeest MI, van der Hulst JM, Melief C], Fleuren G], et al. The detection of circulating human papillomavirus-specific T cells is associated with improved survival of patients with deeply infiltrating tumors. Int ] Cancer. 2011;128(2):37989.

17. Sastre-Garau X, Loste MN, Vincent-Salomon A, Favre M, Mouret E, de la Rochefordiere A, et al. Decreased frequency of HLA-DRB1 13 alleles in Frenchwomen with HPV-positive carcinoma of the cervix. Int ] Cancer. 1996;69(3):159-64.

18. Odunsi K, Ganesan T. Motif analysis of HLA class II molecules that determine the HPV associated risk of cervical carcinogenesis. Int ] Mol Med. 2001;8(4):405-12.

19. Apple R], Erlich HA, Klitz W, Manos MM, Becker TM, Wheeler CM. HLA DR-DQ associations with cervical carcinoma show papillomavirus-type specificity. Nat Genet. 1994;6(2):157-62.

20. Madeleine MM, Brumback B, Cushing-Haugen KL, Schwartz SM, Daling JR, Smith AC, et al. Human leukocyte antigen class II and cervical cancer risk: a population-based study. ] Infect Dis. 2002;186(11):1565-74.

21. Beskow AH, Josefsson AM, Gyllensten UB. HLA class II alleles associated with infection by HPV16 in cervical cancer in situ. Int ] Cancer. 2001;93(6):817-22. 



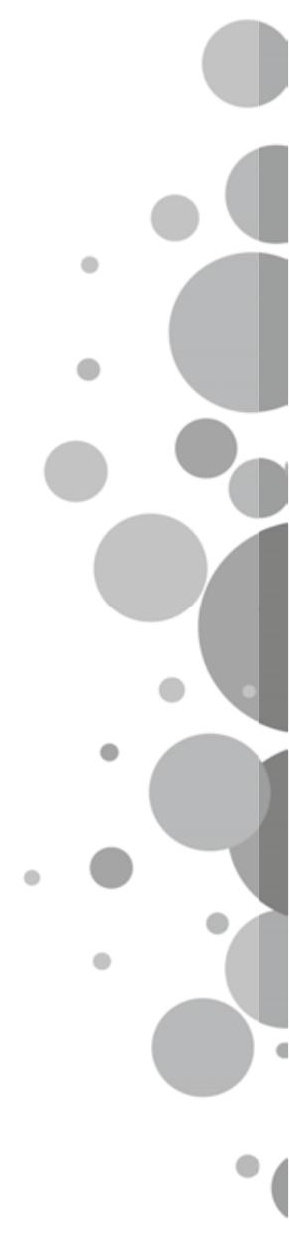




\section{CHAPTER 4}

Gain of chromosomal region $3 q 26$ as a prognostic biomarker for high-grade cervical intraepithelial neoplasia: literature overview and pilot study

Margot M Koeneman, Irene T Ovestad, Emiel AM Janssen, Monique Ummelen, Roy FPM Kruitwagen, Anton H Hopman, Arnold] Kruse

Pathology \& Oncology Research. 2018 Oct 25 


\section{Abstract}

Objective: Approximately $20-40 \%$ of high-grade Cervical Intraepithelial Neoplasia (CIN) regresses spontaneously without treatment, but the natural prognosis of an individual lesion is unpredictable. Gain of the chromosomal 39 region, which contains the human telomerase RNA gene on 3926, is found in CIN lesions and cervical carcinomas and shows correlation with disease grade. The aim of this study is to assess whether 3926 gain as a single genetic marker can predict the natural prognosis of high-grade $\mathrm{CIN}$, by performing a review of the literature and pilot study.

Methods: A literature review was conducted. Additionally, we performed a pilot study in 19 patients with histologically confirmed high-grade CIN lesions who were followed for a mean of 115 days, after which loop excision was performed. Fluorescent in situ hybridization analysis was performed on the initial diagnostic biopsies to determine gain of 3926 .

Results: Eight studies were included in the literature overview, with a total of 407 patients. Of these, only 22 patients had high-grade lesions. All studies found an association between 3926 gain and disease prognosis. Positive predictive values (PPV) ranged from 50-93\%, negative predictive values (NPV) ranged from $75-100 \%$. Only five out of 155 patients (3.2\%) without 3926 gain showed disease persistence or progression. In our pilot study, the PPV of 3926 gain for disease persistence was $67 \%$, the NPV $100 \%$. All four patients without 3926 gain showed disease regression.

Conclusion: The absence of 3926 gain in diagnostic biopsies may be applied to identify highgrade $\mathrm{CIN}$ lesions with a high probability of disease regression. 


\section{Introduction}

High-grade Cervical Intraepithelial Neoplasia (CIN) is caused by Human Papillomavirus (HPV)infection and is considered to be the precursor of cervical carcinoma.[1] Approximately $30 \%$ of high-grade lesions progresses to cervical cancer on the long term, whereas spontaneous regression occurs in approximately $20-40 \%$.[2-6] Conventional histopathological assessment is unable to differentiate between high-grade lesions that will progress to cervical cancer and those that will regress spontaneously. Consequently, most high-grade lesions are currently treated, leading to significant overtreatment with associated side effects. [7] Ideally, the natural prognosis of individual CIN lesions would be predictable, in order to select patients in whom spontaneous regression is expected for a wait-and-see policy.

It has been established that the development of $\mathrm{CIN}$ and concurrent progression to cervical cancer is influenced by a complex interaction between HPV, the host immune system and functional cellular mechanisms. [8, 9] Cervical oncogenesis is characterized by several genetic effects, among which are genomic instability, chromosomal aberrations and integration of viral DNA into the host genome. Markers of these processes have been identified as potential diagnostic or prognostic biomarkers in the diagnosis and prognosis of CIN.[10, 11] Among these is chromosomal region 39 gain, which is frequently found in cervical carcinomas and its precursor lesions. [12] The association between 3q gain and cervical oncogenesis may be caused by amplification of the human telomerase RNA gene (hTERC), which is localized on the 3926 locus. The hTERC gene encodes for the RNA unit of telomerase, which maintains the length of telomeres through cellular divisions. Overexpression of hTERC leads to the avoidance of abnormal cells with critically short telomeres to undergo apoptosis, which is a contributing factor in oncogenesis. Gain of 3926/hTERC or copy number variations has been shown to correlate with disease grade in cervical lesions and could function as a diagnostic tool in cervical pathology.[13-16] Several studies have addressed the prognostic properties of 3926/hTERC gain in the natural prognosis of CIN, but most studies focussed on low-grade lesions and/or evaluated 39 gain in cytological specimen. Evidence on 3q gain in histologically confirmed high-grade $\mathrm{CIN}$ is very scarce. The goal of this study is to provide an overview of the literature on the prognostic properties of 3 q26/hTERC gain in the natural prognosis of $\mathrm{CIN}$ and to investigate the predictive properties of 3926 gain specifically in high-grade CIN.

\section{Materials and methods}

The study was performed according to the PROBE criteria for biomarker research.[17]

\section{Patient population}

For the pilot study, the patient population was extracted from a prospective population based cohort study, conducted at the Stavanger University Hospital, Norway.[5] The women in this cohort were diagnosed with a $\mathrm{CIN} 2$ or $\mathrm{CIN}_{3}$ lesion in a diagnostic biopsy. All biopsies were stained for Hematoxylin Eosin, p16 and Ki67 and disease grade was based on the most severely dysplastic area with the most intensive Ki67 and p16 staining. Staining was assessed for disease grade by 
consensus scoring of three observers (EC, AM, IO), followed by independent quality control of a fourth observer (JB). All used the same microscope ( $40 \times$ objective $0.52 \mathrm{~mm}$, numerical aperture 0.65). In case of discrepancy of $>10 \%$, which occurred in $8 \%$ of all cases, consensus recount was done. All women underwent a Loop Electrosurgical Excision Procedure (LEEP) after a median of 113 days follow-up (range 84-171 days). The natural history of the baseline cervical lesion during the follow-up period was evaluated in the LEEP specimen. Regression was defined as $\mathrm{CIN} 1$ or less in the LEEP specimen. Further details on histological evaluation, HPV genotyping and lesions size measurements can be found in the original article.[5] Out of this cohort, representative and sufficient baseline biopsy material for 3926 analysis was available for 19 patients. These patients were included in the pilot study.

\section{FISH procedure}

FISH analysis was performed on the baseline biopsies. The $3 q$ specific FISH was performed on $4 \mu \mathrm{m}$ thick FFPE tissue sections fixed onto Superfrost Plus Microscope Slides (Thermo Fisher Scientific). The tissue sections were first heated for $15 \mathrm{~min}$ at $80^{\circ} \mathrm{C}$, then dewaxed, hydrated and microwaved for $10 \mathrm{~min}$ at $100^{\circ} \mathrm{C}$ in a $10 \mathrm{mM} \mathrm{Na}$-Citrate $\mathrm{pH}$ buffer and incubated at room temperature for 20 min to cool down. Subsequently, the sections were washed in demineralized water, rinsed in 0.01 $\mathrm{M} \mathrm{HCl}$ and digested with $2.5 \mathrm{mg}$ of pepsin in $0.01 \mathrm{~N} \mathrm{HCl}$ and post-fixed in $1 \%$ formaldehyde in PBS for 5 min at room temperature. Subsequently, the 3 centromere probe ( $p 3.5$ ) and 3926 probe (3q26.1: BAC23 RP11-264D7, Map position 3q26.1 - 26.3 close to the TERC locus), were labeled with Digoxigenin (3c) and Biotin (3q) in a nick translation labelling (Jena Bioscience $\mathrm{CmBH}$, Jena, Germany). The probes were hybridized at a concentration of $2 \mathrm{~g} / \mu \mathrm{l}(3 \mathrm{c}), 5 \mathrm{~g} / \mu \mathrm{l}(3 \mathrm{q}) ; 10 \mathrm{x}$ excess COT, and 75x excess of carrier DNA (salmon sperm DNA) in 50\% formamide; $2 x$ SSC; $10 \%$ dextran sulphate. The probe was applied under a coverslip, simultaneously denatured for $10 \mathrm{~min}$ at $80^{\circ} \mathrm{C}$ and hybridized overnight at $37^{\circ} \mathrm{C}$. After hybridization, the preparations were washed for $5 \mathrm{~min}$ at $61^{\circ} \mathrm{C}$ in a solution, containing $2 \times$ SSC, $0.05 \%$ tween-20 (Janssen Chimica, Beerse, Belgium) and $0.1 \times$ SSC (the washing was carried out twice). The hybridized FISH probe was detected with a triple layer detection method, consisting of 1. FITC-conjugated avidin (Av-FITC, 1:100 dilution, Vector Laboratories) / Monoclonal anti-Digoxigenin (M Dig, 1:100 dilution, Sigma, USA, St Louis MO); 2. Botinylated Coat anti-Avidin (Bio-C A, 1:100 dilution, Vector Laboratories USA) / Rabbit anti Mouse-TRITC (1:100 dilution, Dako, Clostrup, Denmark) and 3. Av-FITC / Swine anti RabbitTRITC (1:100 dilutionDako). Finally, the slides were washed in PBS containing 0.05\% Tween-20, dehydrated in an ascending ethanol series and mounted in Vectashield (Vector Laboratories), containing DAPI (Sigma: $0.5 \mu \mathrm{g} / \mu \mathrm{l}$ ). Images were recorded with the Metasystems Image Pro System (black and white CCD camera; Sandhausen, Germany), mounted on top of a Leica DM-RE fluorescence microscope.[15]

\section{FISH evaluation}

The FISH signals were interpreted by two analysts (MU, AH), who were blinded to the outcome data. Dysplastic areas were identified based on p16 staining and were scanned for FITC and TRITC signal copy numbers. The copy number was estimated as previously described to detect disomy, tetrasomy up to nonasomy by means of the determination of the maximum copy number and heterogeneity in formalin fixed and paraffin embedded tissue sections. The validity of this 
strategy was independently proven by means of a statistical analysis of spot counting in tissue sections. $[18,19]$ Lesions were classified are tetrasomic (copy number 4 ) in case a major fraction exhibited 4 copies, as aneusomic (copy number 3,4 ) in case a major fraction exhibited 3 copies and in case of minor fractions (copy number 2-4). Gain for the targets was noted when dysplastic areas were recognized with more than two copies for $3 c$ or $3 q$. Subsequently, normal morphologic areas were analysed and consistently showed a disomy for 3c and 3q.

\section{Outcome measures and criteria for biomarker performance}

The outcome measure was defined as the correlation between 3926 gain and disease persistence of high-grade CIN. No previous biomarker performance values are available for HLA types. The required test performance values include a high sensitivity and negative predictive value: lesions that will not regress spontaneously must be identified, as treatment is necessary in these women. The actual values depend on the follow-up term of observational management. Lower values can be accepted when strict histological follow-up is implemented to identify persisting lesions at an early stage.

\section{Outcome measure and statistical analysis}

Quantitative variables were described as mean and ranges. Qualitative variables were described as frequency and percentage. Sensitivity, specificity, positive and negative predictive values were calculated from a $2 \times 2$ table. Sample size calculation was not feasible, due to the lack of comparable biomarker performance values and limitation of the study population by the availability of material.

\section{Literature overview}

Eight studies were identified that evaluated the predictive properties of 3q26/hTERC gain in cervical squamous lesions. [20-27] All studies assessed patients with cervical squamous intraepithelial lesions who were followed for a certain period of time, without immediate treatment, in order to evaluate the natural prognosis of the lesions. The main study features are displayed in table 1. Only two studies included patients with high-grade lesions. HeselmeyerHaddad included patients with German PAP 3D cytology, which resembles CIN 1 or 2, of which the latter is interpreted as a high-grade lesion. [20] Ravaioli et al. included five patients with highgrade CIN.[27] 


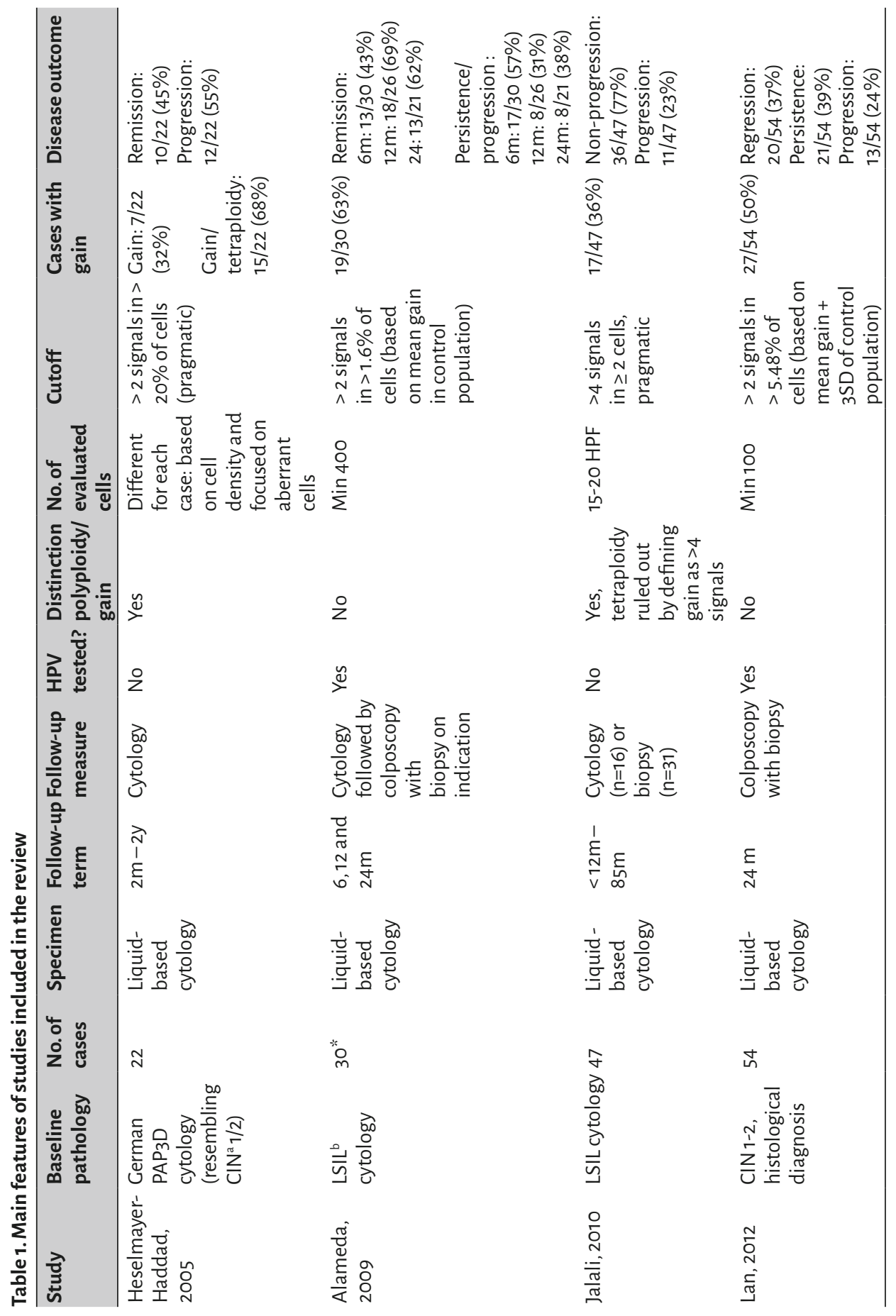




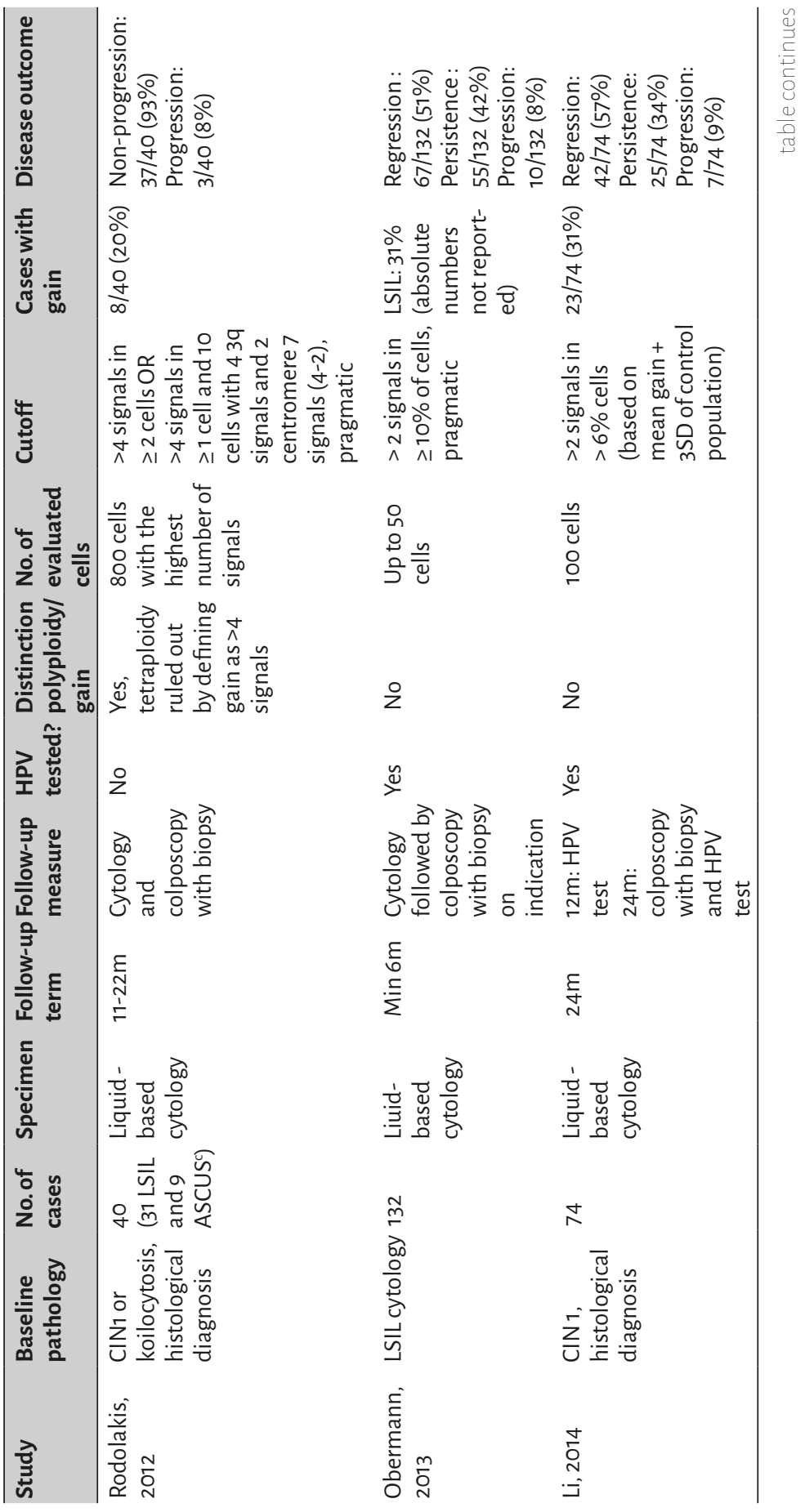




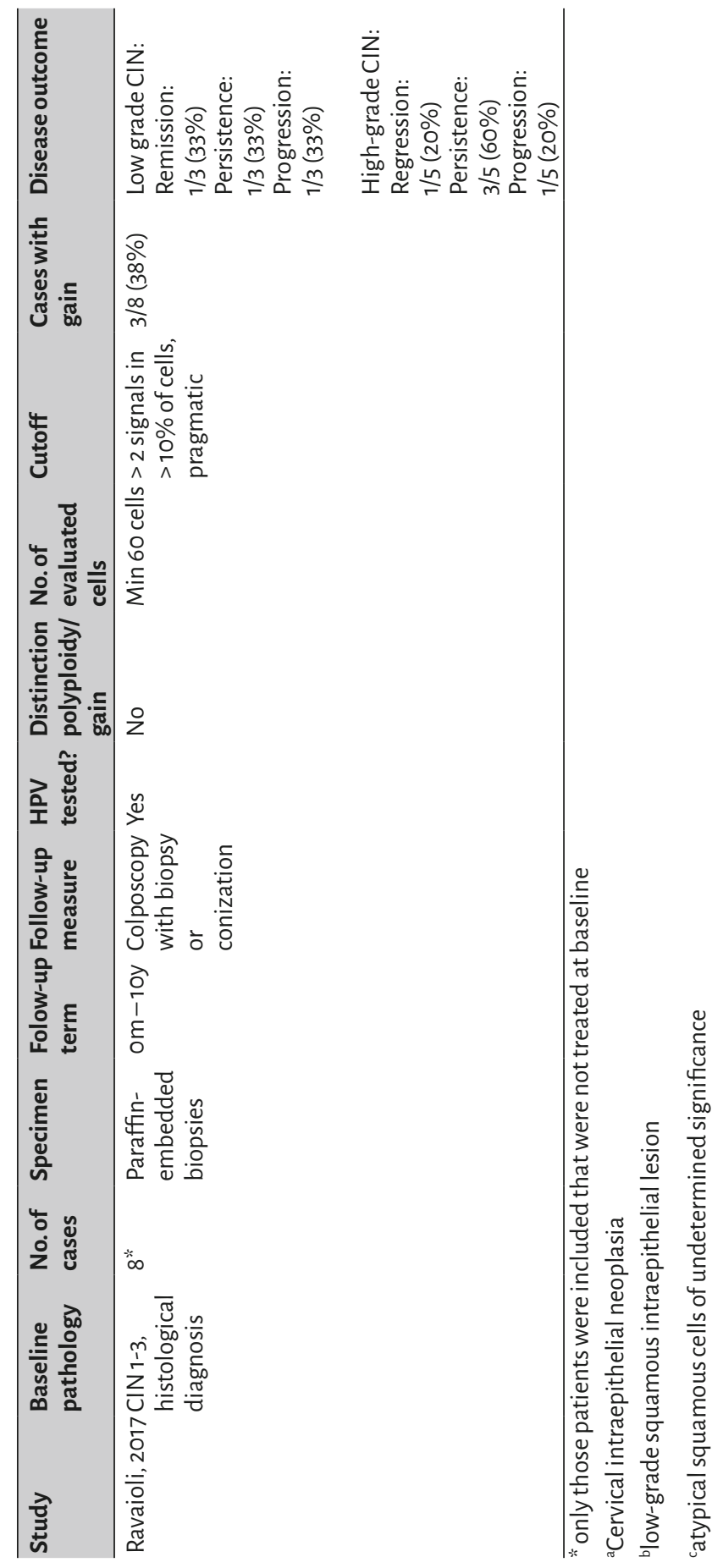




\section{Study results}

The main study results are summarized in table 2. A total of 407 patients were included, of which 385 patients were diagnosed with ASCUS/LSIL/low-grade CIN and 22 patients were diagnosed with HSIL or high-grade CIN. Only five patients had a histological diagnosis of high-grade $\mathrm{CIN}$. Pooling of the study results was not possible, due to a marked heterogeneity in patient populations, follow-up terms and outcome-measures. Only five out of 155 patients (3.2\%) without $3 q 26$ gain showed disease persistence or progression.

Table 2. Results of studies on 3q26/hTERC gain as a prognostic biomarker in CIN

\begin{tabular}{|c|c|c|c|c|}
\hline \multirow[t]{2}{*}{ Author } & \multirow[t]{2}{*}{$\begin{array}{l}\text { Gain of } 3926 / h T E R C \\
\text { per outcome group } \\
\text { (using study threshold) }\end{array}$} & \multirow[t]{2}{*}{$\begin{array}{l}\text { Progression in } \\
\text { gain-negative } \\
\text { group }\end{array}$} & \multicolumn{2}{|l|}{ Test properties } \\
\hline & & & $\begin{array}{l}\text { Prediction of persistencel } \\
\text { progression vs regression }\end{array}$ & $\begin{array}{l}\text { Prediction of progression } \\
\text { vs regression/persistence }\end{array}$ \\
\hline $\begin{array}{l}\text { Heselmayer- } \\
\text { Haddad, } \\
2005\end{array}$ & $\begin{array}{l}\text { Only } 3926 \text { gain: } \\
\text { Progression: } 7 / 12 \\
\text { Regression: } 0 / 10 \\
\text { 3q26 gain and/or } \\
\text { tetraploidy: } \\
\text { Progression: } 12 / 12 \\
\text { Regression: } 3 / 10\end{array}$ & $5 / 15$ & $\begin{array}{l}\text { Sens }^{\mathrm{a}} 100 \% \\
\text { Spec }^{\mathrm{b}} 70 \% \\
\text { PPV }^{\mathrm{c}} 80 \% \\
\text { NPV }^{d} 100 \% \\
\text { (progression vs regression, } \\
\text { gain+ tetraploidy group) }\end{array}$ & \\
\hline $\begin{array}{l}\text { Alameda, } \\
2009\end{array}$ & $\begin{array}{l}\frac{6 \text { months }}{\text { Regression: } 7 / 15} \\
\text { Persistence/ progression: } \\
\text { 12/15 } \\
\frac{12 / 24 \text { months }}{\text { Regression: } 8 / 18} \\
\text { Persistence/ progression: } \\
6 / 8\end{array}$ & NR & $\begin{array}{l}\frac{6 \text { months }}{\text { Sens } 80 \%} \\
\text { Spec 53\% } \\
\text { PPV 63\% } \\
\text { NPV 73\% } \\
12 / 24 \text { months } \\
\text { Sens } 75 \% \\
\text { Spec 53\% } \\
\text { PPV } 43 \% \\
\text { NPV } 91 \%\end{array}$ & \\
\hline Jalali, 2010 & $\begin{array}{l}\text { Regression/ persistence: } \\
\text { 7/36 } \\
\text { Progression: } 10 / 11\end{array}$ & $1 / 30$ & & $\begin{array}{l}\text { Sens } 91 \% \\
\text { Spec } 81 \% \\
\text { PPV 59\% } \\
\text { NPV } 97 \%\end{array}$ \\
\hline Lan, 2012 & $\begin{array}{l}\text { Regression: } 2 / 20 \\
\text { Persistence: } 12 / 21 \\
\text { Progression: } 13 / 13\end{array}$ & $0 / 27$ & $\begin{array}{l}\text { Sens 74\% } \\
\text { Spec 90\% } \\
\text { PVV 93\% } \\
\text { NPV 67\% }\end{array}$ & $\begin{array}{l}\text { Sens } 100 \% \\
\text { Spec } 66 \% \\
\text { PPV } 48 \% \\
\text { NPV } 100 \%\end{array}$ \\
\hline $\begin{array}{l}\text { Rodolakis, } \\
2012\end{array}$ & $\begin{array}{l}\text { Regression/ persistence: } \\
\text { 5/37 } \\
\text { Progression: } 3 / 3\end{array}$ & $0 / 32$ & & $\begin{array}{l}\text { Sens 100\% } \\
\text { Spec } 89 \% \\
\text { PPV } 50 \% \\
\text { NPV } 100 \%\end{array}$ \\
\hline
\end{tabular}




\begin{tabular}{|c|c|c|c|c|}
\hline \multirow[t]{2}{*}{ Author } & \multirow{2}{*}{$\begin{array}{l}\text { Gain of 3q26/hTERC } \\
\text { per outcome group (using } \\
\text { study threshold) }\end{array}$} & \multirow{2}{*}{$\begin{array}{l}\text { Progression in } \\
\text { gain-negative } \\
\text { group }\end{array}$} & \multicolumn{2}{|l|}{ Test properties } \\
\hline & & & $\begin{array}{l}\text { Prediction of persistence/ } \\
\text { progression vs regression }\end{array}$ & $\begin{array}{l}\text { Prediction of progression } \\
\text { vs regression/ persistence }\end{array}$ \\
\hline $\begin{array}{l}\text { Obermann, } \\
2013\end{array}$ & $\begin{array}{l}\text { Regression: } 16 / 67 \\
\text { Persistence: } 15 / 55 \\
\text { Progression: } 7 / 10\end{array}$ & $3 / 54$ & $\begin{array}{l}\text { Sens } 70 \% \\
\text { Spec } 76 \% \\
\text { PPV } 30 \% \\
\text { NPV } 94 \% \\
\text { (progression vs regression) }\end{array}$ & $\begin{array}{l}\text { Sens } 35 \% \\
\text { Spec } 76 \% \\
\text { PPV 58\% } \\
\text { NPV } 54 \%\end{array}$ \\
\hline Li, 2014 & $\begin{array}{l}\text { Regression: } 4 / 42 \\
\text { Persistence/ progression: } \\
19 / 32\end{array}$ & NR & $\begin{array}{l}\text { Sens } 59 \% \\
\text { Spec } 90 \% \\
\text { PPV } 82 \% \\
\text { NPV } 75 \%\end{array}$ & \\
\hline $\begin{array}{l}\text { Ravaioli, } \\
2017\end{array}$ & $\begin{array}{l}\text { Regression: } 0 / 2 \\
\text { Persistence: } 2 / 4 \\
\text { Progression: } 1 / 2\end{array}$ & $1 / 5$ & $\mathrm{NA}^{\mathrm{e}}$ & \\
\hline
\end{tabular}

${ }^{a}$ sensitivity, ${ }^{b}$ specificity, ${ }^{\text {cppositive }}$ redictive value, ${ }^{d}$ negative predictive value, ${ }^{e}$ not applicable

\section{Summary and appraisal}

All studies identify 3q26/hTERC gain as a potential prognostic marker in cervical precancerous lesions. 3q26/hTERC gain seems more frequent in persistent or progressive lesions, but positive predictive values are generally low: patients with 3q26/hTERC gain often show disease regression during follow-up. Negative predictive values are consistently higher: absence of 3q26/hTERC gain seems to be a strong predictor of disease regression.

Nevertheless, several limitations of the individual studies and their review must be noted. Patient populations were generally small. Most studies included only patients with low-grade lesions, which limits the evidence on the prognostic properties of 3q26/hTERC gain in high-grade lesions. The baseline diagnosis was not determined uniformly: some studies included patients based on cytology, whereas others included only histologically confirmed lesions. Furthermore, followup periods and methods differed: both cytology and histology was applied. Regarding the FISH analysis, the different studies did not apply a similar signal interpretation method and threshold for gain. Another important limitation in the interpretation of the study results is that HPV testing was not performed or reported in most studies. It is therefore unclear whether all lesions were HPV-induced. This limits the applicability of the study results to high-grade lesions, which are usually HPV positive. Moreover, HPV genotype is an individual predictor in the natural history of CIN lesions. As such, information on HPV status would improve the interpretation of the study data.

Despite all limitations, 3926/hTERC analysis has been consistently identified as a prognostic marker in cervical precancerous lesions, with a high negative predictive value in mostly low-grade lesions. As such, evidence indicates a potential predictive role of 3926/hTERC gain in the natural prognosis of cervical dysplasia, but clinical applicability is yet limited and the evidence on the 
predictive properties of 3926/hTERC gain in histologically confirmed high-grade lesions is scarce $(n=5)$. This prompted us to perform a pilot study evaluating the predictive properties of 3926/ hTERC gain in histologically confirmed high-grade lesions.

\section{Pilot study: results}

\section{Patient characteristics}

A total of 19 women were included in our study. The mean age was 31 years (range 25-41). The mean interval between the initial colposcopy and the follow-up colposcopy with LEEP was 115 days (91-154 days). Nine women (47\%) showed disease regression during this follow-up period and ten did not (53\%). The mean age and biopsy-LEEP interval did not differ significantly between women who showed spontaneous regression and those who did not (mean age 32 vs 31 years, mean biopsy-LEEP interval 115 vs 114 days respectively).

\section{Lesions characteristics: CIN grade, lesions size, HPV genotyping and p16 staining}

Sixteen patients were diagnosed with a $\mathrm{CIN}_{3}$ lesion and three with a $\mathrm{CIN} 2$ lesion. Of the patients with a $\mathrm{CIN} 2$ lesion, two showed regression and one showed disease persistence (22\% vs $10 \%$, $\mathrm{p}=0.5$ ). In the original study, lesions were classified according to size in two categories: larger than $2.5 \mathrm{~mm}$ or equal to or smaller than $2.5 \mathrm{~mm}$. There was no difference between these two categories in terms of the number of women with and without regression: a lesion larger than $2.5 \mathrm{~mm}$ was found in $5 / 9$ (56\%) women with disease regression and in 7/10 (70\%) women with disease persistence $(p=0.54)$. All women carried high-risk HPV. HPV16 was found in 5/9 (56\%) women with disease regression and $7 / 10(70 \%)$ women with disease persistence $(p=0.54)$. The biopsy material of all cases was 16 positive, confirming HPV infection.

\section{FISH results}

Results of the 3926 analysis are shown in table 3. Figure 1 shows typical examples of the FISH analysis. Four patients showed no 3926 gain, all of their lesions regressed spontaneously. Of interest, all $\mathrm{CIN} 2$ lesions showed 3926 gain. The test performance of 3926 gain in the prediction of natural prognosis of high-grade CIN in the study population is shown in table 4 . When the analysis was restricted to only $\mathrm{CIN} 3$ lesions, the positive predictive value increased to $75 \%$, while the negative predictive value remained $100 \%$. 
Table 3. Results of 3926 analysis and natural prognosis in 19 patients with high-grade CIN

\begin{tabular}{llllll}
\hline Case & CIN grade & $3 q 26$ copy numbers & $\mathbf{3 c}$ copy numbers & 3q status & $\begin{array}{l}\text { Regression } \\
\text { (yes/no) }\end{array}$ \\
\hline 96 & 3 & 4 & 4 & Gain & No \\
125 & 3 & 3,4 & 3,4 & Gain & No \\
159 & 3 & 4 & 4 & Gain & No \\
164 & 3 & 4 & 4 & Gain & No \\
170 & 3 & $2-4$ & $2-4$ & Gain & No \\
171 & 3 & $2-6$ & $2-6$ & Gain & No \\
182 & 3 & 3,4 & 3,4 & Gain & No \\
218 & 2 & $6-8$ & 3,4 & Gain & No \\
221 & 3 & $2-4$ & $2-4$ & Gain & No \\
225 & 3 & $2-4$ & $2-4$ & Gain & No \\
187 & 2 & $2-4$ & $2-4$ & Gain & Yes \\
192 & 3 & 4 & 4 & Gain & Yes \\
207 & 3 & 3 & 3 & Gain & Yes \\
222 & 3 & $2-4$ & $2-4$ & Gain & Yes \\
237 & 2 & 3,4 & 3,4 & Gain & Yes \\
194 & 3 & 2 & 2 & No gain & Yes \\
197 & 3 & 2 & 2 & No gain & Yes \\
200 & 3 & 2 & 2 & No gain & Yes \\
206 & 3 & 2 & 2 & No gain & Yes \\
\hline
\end{tabular}

Table 4. Test performance of 3926 gain in the prediction of natural prognosis of high-grade CIN in 19 patients

\begin{tabular}{llll} 
& Persistence & Regression & Total \\
\cline { 2 - 4 } Gain of 3q & 10 & 5 & 15 \\
No gain of 3q & 0 & 4 & 4 \\
Total & 10 & 9 & 19 \\
\cline { 1 - 2 }$=0.0325$ & & & \\
Sensitivity: & & $100 \%(95 \% \mathrm{Cl} 66-100 \%)$ & \\
Specificity: & & $44 \%(95 \% \mathrm{Cl} 15-77 \%)$ & \\
Positive predictive value: & $67 \%(95 \% \mathrm{Cl} 39-87 \%)$ & \\
Negative predictive value: & $100 \%(95 \% \mathrm{Cl} 40-100 \%)$ & \\
\hline
\end{tabular}



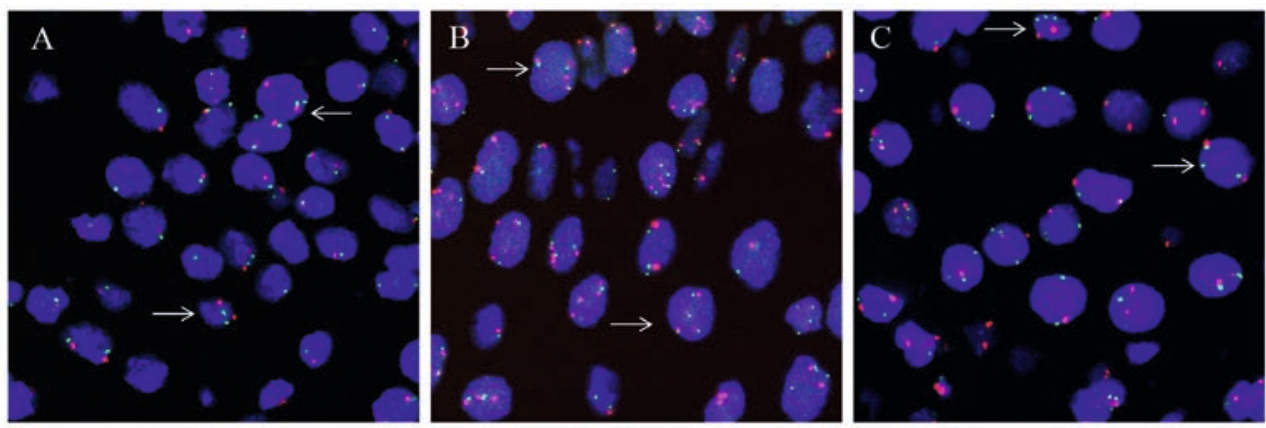

Figure 1. Fluorescence in situ hybridization in high-grade CIN

Typical examples showing a disomy in A) for both 3926 (green FITC signal) and 3 C (red TRITC signal) (case number 197). In B) a tetrasomy (case 192) and in C) an imbalance between 3926 and $3 c$ (case 218). In the latter case the cells showed a ratio of 3 to 2 signals for 3926 and 3 c respectively, with nuclei with multiple copies for both targets (classified as gain). The arrows point to the nuclei with the typical signal distribution for the cases with no gain $(A)$ and gain $(B, C)$.

\section{Discussion}

This is the first study to assess the prognostic value of 3926 gain as a single genetic marker in the natural prognosis of solely high-grade CIN. The results show that 3926 gain is found in both women with persistence and regression of high-grade $\mathrm{CIN}$, but that none of the women without 3926 gain show disease persistence. This results in a high negative predictive value of 3926 for disease persistence. As such, the absence of $3926 / \mathrm{hTERC}$ gain may potentially be applied to identify those lesions with a high potential of disease regression.

The test performance of hTERC gain in high-grade lesions in our pilot study is comparable to the test performance of hTERC gain in low-grade lesions, as reviewed in the literature. Negative predictive values were consistently high, while positive predictive values were much lower. The mediocre positive predictive value of 3926/hTERC gain as a prognostic marker for disease persistence and/or progression may indicate that hTERC gain is a contributing, but not critical step in cervical oncogenesis. The development of cervical precancerous lesions and subsequent carcinoma is based on a complex interaction between virus and host, in which viral oncogenic properties and the human immune system influence the cellular processes that lead to cell transformation. [8, 9] In this process, several important molecular events have been identified, among which are viral DNA integration and upregulation of telomerase.[8] However, none of these events have been identified as critical steps or 'point of no return'. Indeed, upregulation of telomerase is not found in all high-grade CIN lesions or cervical carcinomas. [12] As such, it is unlikely that the prediction of the natural prognosis of CIN lesions will be based on one molecular event, but rather on a combination of viral, host and genetic parameters. Therefore, combining hTERC testing with other predictive biomarkers may lead to a test panel with a better overall test performance. 
Interestingly, 3q gain can occur based on tetrasomy, in which four copy numbers are found, or aneusomy, in which three or more than four copy numbers are found. It is unclear whether there is a clinical difference between these two forms of 39 gain, in terms of the risk of disease persistence or progression. Both tetrapoidy and aneuploidy are frequent events in CIN development. The frequency of tetraploid cells is significantly increased in CIN lesions compared to normal cervical tissue and is considered an early event in cervical carcinogenesis.[28] Aneuploidy is more often found in more advanced lesions and cervical carcinoma.[15] Although it is still debated whether aneuploidy results from genomic instability of diploid cells or from chromosomal losses from tetraploid cells, evidence in CIN lesions suggests that aneuploidy is preceded by tetraploidy. [28] This would indicate that aneuploidy in CIN lesions associates with later stages of cervical oncogenesis, possibly indicated a more high-risk CIN lesion. Based on these findings, one may argue that 3q26/hTERC gain based on aneuploidy imposes a greater risk of disease persistence or progression than 3926/hTERC gain based on tetraploidy. In our study, the only purely aneusomic lesion showed disease regression. Our study shows no difference in disease regression or persistence based on 3926 gain in tetrasomic or aneusomic lesions, but numbers are small and the follow-up term was relatively short. The reviewed studies show conflicting results regarding the prognostic value of 39 gain based on either aneuploidy or tetraploidy. One study showed a positive predictive value of $100 \%$ for gain based on aneuploidy for disease progression.[20] Two other studies could not confirm this finding, but compared progression to non-progression (including persistence), which makes comparison of the studies difficult.[22, 24] Interestingly, Lan et al. found a higher progression risk for tetraploid lesions.[23] In conclusion, current evidence shows that lesions with 3926 gain based on both tetrasomy and aneusomy can show either regression, persistence or progression. Based on these results, it remains unclear whether there is a clinical difference between 3q26/hTERC gain based on tetrasomy or aneusomy, in terms of the risk of disease persistence or progression.

Only five out of eight reviewed studies performed HPV typing, of which only one study reported on the association between HPV and 39 gain: a non-significant association was found between viral load and 3q gain. [21] As discussed before, this limits the overall interpretation of the study results, since high-risk HPV in itself is a risk factor for disease progression/persistence. Regarding the relation between HPV infection and 3q26/hTERC gain, it is debated whether 3q26/hTERC gain is a direct cause of HPV infection, or an independent risk factor in high-grade CIN. It has been shown that genomic integration of HPV (with increased expression of E6 and E7) and gain of hTERC are important associated genetic events in the progression of CIN to cervical cancer.[16] On the other hand, 3q gains have also been detected in non-HPV-associated squamous cell cancers of the lower genital tract and other malignancies. [12] Assessment of HPV status is therefore vital in future studies on the prognostic properties of 3926/hTERC. Furthermore, future studies should clarify the association between HPV genotype and 39 gain, with regard to the natural prognosis of high-grade $\mathrm{CIN}$.

Limitations of the current clinical study include the small patient population. The patient population was extracted from a historical cohort of patients from a previous study, based upon the availability of sufficient biopsy material. Another limitation of our study may be the use of 
histological specimen instead of cytology for the FISH analysis, which has been shown to be more sensitive to the identification of cells with 39 gain. $[12,20]$ We however chose to perform FISH analysis in biopsy material, as histology is the golden standard for a CIN diagnosis. A limitation regarding the interpretation of the study results, is the effect of a diagnostic biopsy on the natural history of the lesion. It is suggested that the biopsy itself may induce lesions regression. This would limit the interpretation of any prognostic marker, and applies to all studies on the natural history of CIN lesions. On the other hand, high-grade lesions are clinically diagnosed with a biopsy, making the prognostic effect of a prognostic biomarker clinically applicable despite the effect of the biopsy on regression itself. Another general limitation with regard to the interpretation and application of histological biomarkers in high-grade $\mathrm{CIN}$ is the possibility of false negative results due to sampling error, in which the biopsy is not representative of the actual disease status. We therefore propose that histological biomarkers should be applied as part of a biomarker profile, which should also contain biomarkers that are independent of the disease histology. Examples are cytological, serological or epidemiological markers, such as HPV-genotype, immune markers and smoking status.

In conclusion, the results of the current review and pilot study show that the absence of 3q26 gain could potentially serve as a prognostic biomarker for the identification of CIN lesions with a high probability of disease regression, preferentially as part of a broader biomarker profile. As such, 3926 staining could aid in the selection of women with low-grade lesions who would not need immediate colposcopic assessment and women with high-grade lesions who would not need immediate treatment. Both strategies could result in reduced costs, patient burden and side effects of surgical treatment. To confirm this hypothesis further research is necessary. Research should focus on identification of a generalized methodology for 3926 gain testing and interpretation. Subsequently, its prognostic properties should be confirmed in a larger patient population. Moreover, assessment of the association between HPV and $3 q 26$ gain is needed. Upon confirmation of its prognostic properties, 3926 staining could be considered as part of a biomarker profile to triage women with high-grade lesions for conservative follow-up measures. 


\section{References}

1. zur Hausen H. Papillomaviruses causing cancer: evasion from host-cell control in early events in carcinogenesis. J Natl Cancer Inst. 2000;92(9):690-8.

2. McCredie MR, Sharples K], Paul C, Baranyai J, Medley G, Jones RW, et al. Natural history of cervical neoplasia and risk of invasive cancer in women with cervical intraepithelial neoplasia 3: a retrospective cohort study. Lancet Oncol. 2008;9(5):425-34.

3. Trimble CL, Piantadosi S, Gravitt P, Ronnett B, Pizer E, Elko A, et al. Spontaneous regression of high-grade cervical dysplasia: effects of human papillomavirus type and HLA phenotype. Clin Cancer Res. 2005;11(13):4717-23.

4. Ovestad IT, Gudlaugsson E, Skaland I, Malpica A, Munk AC, Janssen EA, et al. The impact of epithelial biomarkers, local immune response and human papillomavirus genotype in the regression of cervical intraepithelial neoplasia grades 2-3. J Clin Pathol. 2011;64(4):303-7.

5. Munk AC, Gudlaugsson E, Ovestad IT, Lovslett K, Fiane B, Hidle B, et al. Interaction of epithelial biomarkers, local immune response and condom use in cervical intraepithelial neoplasia 2-3 regression. Gynecol Oncol. 2012;127(3):489-94.

6. Grimm C, Polterauer S, Natter C, Rahhal ], Hefler L, Tempfer CB, et al. Treatment of cervical intraepithelial neoplasia with topical imiquimod: a randomized controlled trial. Obstet Gynecol. 2012;120(1):152-9.

7. Barken SS, Rebolj M, Andersen ES, Lynge E. Frequency of cervical intraepithelial neoplasia treatment in a well-screened population. Int ] Cancer. 2012;130(10):2438-44.

8. Moody CA, Laimins LA. Human papillomavirus oncoproteins: pathways to transformation. Nat Rev Cancer. 2010;10(8):550-60.

9. Stanley MA. Epithelial cell responses to infection with human papillomavirus. Clin Microbiol Rev. 2012;25(2):215-22.

10. Litjens R], Hopman AH, van de Vijver KK, Ramaekers FC, Kruitwagen RF, Kruse A]. Molecular biomarkers in cervical cancer diagnosis: a critical appraisal. Expert Opin Med Diagn. 2013;7(4):365-77.

11. Koeneman MM, Kruitwagen RF, Nijman HW, Slangen BF, Van Corp T, Kruse A]. Natural history of high-grade cervical intraepithelial neoplasia: a review of prognostic biomarkers. Expert Rev Mol Diagn. 2015;15(4):527-46.

12. Thomas LK, Bermejo JL, Vinokurova S, Jensen K, Bierkens $M$, Steenbergen R, et al. Chromosomal gains and losses in human papillomavirus-associated neoplasia of the lower genital tract - a systematic review and meta-analysis. Eur ] Cancer. 2014;50(1):85-98.

13. Seppo A, Jalali GR, Babkowski R, Symiakaki H, Rodolakis A, Tafas T, et al. Gain of 3q26: a genetic marker in low-grade squamous intraepithelial lesions (LSIL) of the uterine cervix. Gynecol Oncol. 2009;114(1):80-3.

14. Earley A, Lamont JL, Dahabreh I], Cowan J, Feldman S, Uhlig K. Fluorescence in situ hybridization testing for the diagnosis of high-grade cervical abnormalities: a systematic review. J Low Genit Tract Dis. 2014;18(3):218-27.

15. Hopman AH, Theelen W, Hommelberg PP, Kamps MA, Herrington CS, Morrison LE, et al. Cenomic integration of oncogenic HPV and gain of the human telomerase gene TERC 
at 3926 are strongly associated events in the progression of uterine cervical dysplasia to invasive cancer. ] Pathol. 2006;210(4):412-9.

16. Wright TC, Compagno ], Romano P, Grazioli V, Verma Y, Kershnar E, et al. Amplification of the 39 chromosomal region as a specific marker in cervical cancer. Am ] Obstet Gynecol. 2015;213(1):51 e1-8.

17. Pepe MS, Feng Z, Janes H, Bossuyt PM, Potter JD. Pivotal evaluation of the accuracy of a biomarker used for classification or prediction: standards for study design. ] Natl Cancer Inst. 2008;100(20):1432-8.

18. Hopman AH, van Hooren E, van de Kaa CA, Vooijs PG, Ramaekers FC. Detection of numerical chromosome aberrations using in situ hybridization in paraffin sections of routinely processed bladder cancers. Mod Pathol. 1991;4(4):503-13.

19. Pahlplatz MM, de Wilde PC, Poddighe P, van Dekken H, Vooijs GP, Hanselaar AC. A model for evaluation of in situ hybridization spot-count distributions in tissue sections. Cytometry. 1995;20(3):193-202.

20. Heselmeyer-Haddad K, Sommerfeld K, White NM, Chaudhri N, Morrison LE, Palanisamy N, et al. Cenomic amplification of the human telomerase gene (TERC) in pap smears predicts the development of cervical cancer. Am J Pathol. 2005;166(4):1229-38.

21. Alameda F, Espinet B, Corzo C, Munoz R, Bellosillo B, Lloveras B, et al. 3926 (hTERC) gain studied by fluorescence in situ hybridization as a persistence-progression indicator in lowgrade squamous intraepithelial lesion cases. Hum Pathol. 2009;40(10):1474-8.

22. Jalali GR, Herzog T], Dziura B, Walat R, Kilpatrick MW. Amplification of the chromosome $3 q 26$ region shows high negative predictive value for nonmalignant transformation of LSIL cytologic finding. Am J Obstet Gynecol. 2010;202(6):581 e1-5.

23. Lan YL, Yu L, Jia CW, Wu YM, Wang SY. Gain of human telomerase RNA gene is associated with progression of cervical intraepithelial neoplasia grade I or II. Chin Med ] (Engl). 2012;125(9):1599-602.

24. Rodolakis A, Biliatis I, Symiakaki H, Kershnar E, Kilpatrick MW, Haidopoulos D, et al. Role of chromosome 3926 gain in predicting progression of cervical dysplasia. Int ] Gynecol Cancer. 2012;22(5):742-7.

25. Obermann EC, Savic Prince S, Barascud A, Grilli B, Herzog M, Kaup D, et al. Prediction of outcome in patients with low-grade squamous intraepithelial lesions by fluorescence in situ hybridization analysis of human papillomavirus, TERC, and MYC. Cancer Cytopathol. 2013;121(8):423-31.

26. Li L, Jiang W, Zeng SY, Li L. Prospective study of hTERC gene detection by fluorescence in situ hybridization (FISH) in cervical intraepithelial neoplasia 1 natural prognosis. Eur ] Gynaecol Oncol. 2014;35(3):289-91.

27. Ravaioli S, Tumedei MM, Amadori A, Puccetti M, Chiadini E, Bravaccini S. Role of Telomerase in Cervical Lesions as Prognostic Marker: A Comparison Between Immunohistochemistry and Fluorescence In Situ Hybridization. J Low Genit Tract Dis. 2017;21(1):42-6.

28. Olaharski A], Sotelo R, Solorza-Luna G, Gonsebatt ME, Guzman P, Mohar A, et al. Tetraploidy and chromosomal instability are early events during cervical carcinogenesis. Carcinogenesis. 2006;27(2):337-43. 


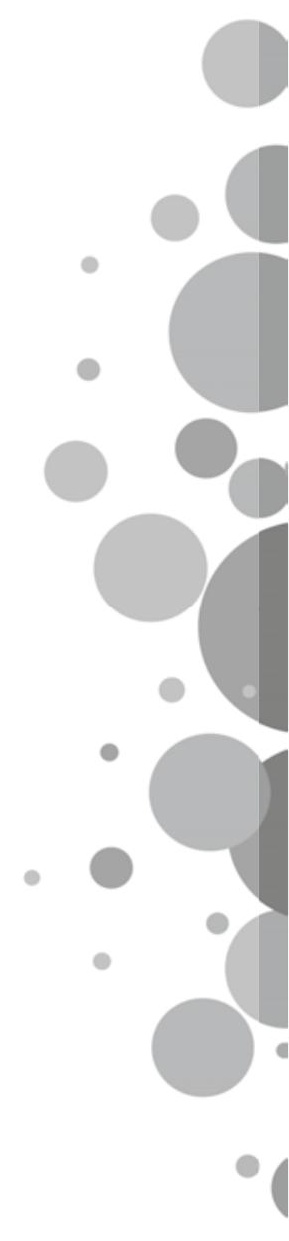




\section{CHAPTER 5}

A prediction model for spontaneous regression of cervical intraepithelial neoplasia grade 2 , based on simple clinical parameters

Margot M Koeneman, Freyja HM van Lint, Sander M] van Kuijk, Luc]M Smits, Loes FS Kooreman, Roy FPM Kruitwagen, Arnold] Kruse

Human Pathology. 2017]an;59:62-69. 


\begin{abstract}
This study aims to develop a prediction model for spontaneous regression of cervical intraepithelial neoplasia grade 2 ( $\mathrm{CIN}$ 2) lesions based on simple clinical and pathological parameters. The study was conducted at Maastricht University Medical Center, the Netherlands. The prediction model was developed in a retrospective cohort of 129 women with a histological diagnosis of CIN 2 who were managed by watchful waiting for 6-24 months. Five potential predictors for spontaneous regression were selected based on the literature and expert opinion and were analyzed in a multivariable logistic regression model, followed by backward stepwise deletion based on the Wald test. The prediction model was internally validated by the bootstrapping method. Discriminative capacity and accuracy were tested by assessing the area under the receiver operating characteristic curve (AUC) and a calibration plot. Disease regression within 24 months was seen in 91 out of 129 (71\%) patients. A prediction model was developed including the following variables: smoking, PAP smear outcome prior to the CIN 2 diagnosis, concomitant $\mathrm{CIN} 1$ diagnosis in the same biopsy, and more than one biopsy containing $\mathrm{CIN}$ 2. Not smoking, $<\mathrm{PAP}_{3}$, concomitant $\mathrm{CIN} 1$ and no more than one biopsy containing CIN 2 were predictive of disease regression. The AUC was 69.2\% [95\% confidence interval (Cl), 58.5-79.9\%], indicating a moderate discriminative ability of the model. The calibration plot indicated good calibration of the predicted probabilities. This prediction model for spontaneous regression of CIN 2 may aid physicians in the personalized management of these lesions.
\end{abstract}




\section{Introduction}

High-grade cervical intraepithelial neoplasia (CIN 2-3, High-Grade Squamous Intraepithelial Lesion, HSIL) is considered to be the precursor of cervical carcinoma. However, not all high-grade $\mathrm{CIN}$ lesions will develop into cervical cancer; indeed, prospective follow-up studies show that a substantial subset of high-grade CIN lesions will regress spontaneously without treatment $[1,2]$. Treatment guidelines have historically advised surgical treatment of high-grade CIN (CIN 2-3), most commonly by large loop excision of the transformation zone (LLETZ). Since a substantial percentage of high-grade CIN lesions show spontaneous regression, this standardized treatment policy leads to overtreatment and unnecessary complications as a result of surgical excision. The most serious long-term complication of LLETZ is an increased risk of premature birth in subsequent pregnancies, most probably as a result of cervical insufficiency [3, 4]. Since CIN 2 has a higher spontaneous regression rate than $\mathrm{CIN}_{3}$, overtreatment is most profound for CIN 2 lesions [5]. For this reason, conservative management of $\mathrm{CIN} 2$ for young women was previously proposed by other authors [6]. International guidelines also advocate conservative treatment of younger women with $\mathrm{CIN} 2$ [7, 8]. As a result, LLETZ rates have decreased in patients with CIN 2 histology preceded by low-grade abnormal cytology [9].

Ideally, those lesions that will regress spontaneously should be differentiated from those that will not, allowing for watchful waiting instead of invasive treatment in the subgroup of patients in which spontaneous regression is expected. Prediction of the individual probability of regression of high-grade CIN is essential for this strategy. Conventional histopathological assessment is unfit for this purpose. Several patient and biopsy characteristics and immunological or histological biomarkers have been identified that correlate with prognosis in high-grade CIN, but no biomarker or biomarker profile has yet reached clinical applicability [10]. This study aims to develop a widely applicable prediction model for spontaneous regression of CIN 2 lesions based on patient and lesion characteristics, as these are simple and readily available clinical parameters.

\section{Methods}

\section{Setting}

This registration-based retrospective cohort study was conducted at the Maastricht University Medical Center, Maastricht, the Netherlands. Approval for this study was obtained from the Medical Ethics Committee of Maastricht University Medical Center (approval number: METC 154-174; September 2, 2015).

\section{Population}

Patients with a diagnosis of CIN 2 from a diagnostic biopsy obtained during a colposcopy who received observational management (including cytological follow-up) between January 1, 2000 and April 30, 2013 were retrospectively identified and assessed for eligibility. According to the colposcopy protocol, biopsies were taken from each suspicious lesion. The histopathological assessment of cervical biopsies was performed according to national guidelines, based on the World Health Organization criteria, with p16 staining in case of an inconclusive diagnosis based 
on hematoxylin and eosin staining (figure 1). Observational management of CIN 2 consisted of serial PAP smears after 6,12 , and 24 months. Patients were included in this study if they had at least one follow-up visit with cervical cytology during the first year. A new colposcopy was planned in case of PAP 3 a2 (cytological HSIL) or higher at either of the follow-up visits. Exclusion criteria were previous treatments for high-grade $\mathrm{CIN}$ and concomitant $\mathrm{CIN} 3$ or higher. For patients who completed the follow-up schedule, disease regression was defined as PAP 1 cytology at the 24 month follow-up or $\leq \mathrm{CIN} 1$ histology at the 24 month follow-up and no diagnosis of $>\mathrm{CIN} 2$ before the 24 month follow-up visit. For patients who did not complete the 24 month follow-up schedule, those with PAP 1 cytology at the last follow-up visit were included in the regression group, and all other patients were included in the persistence group.
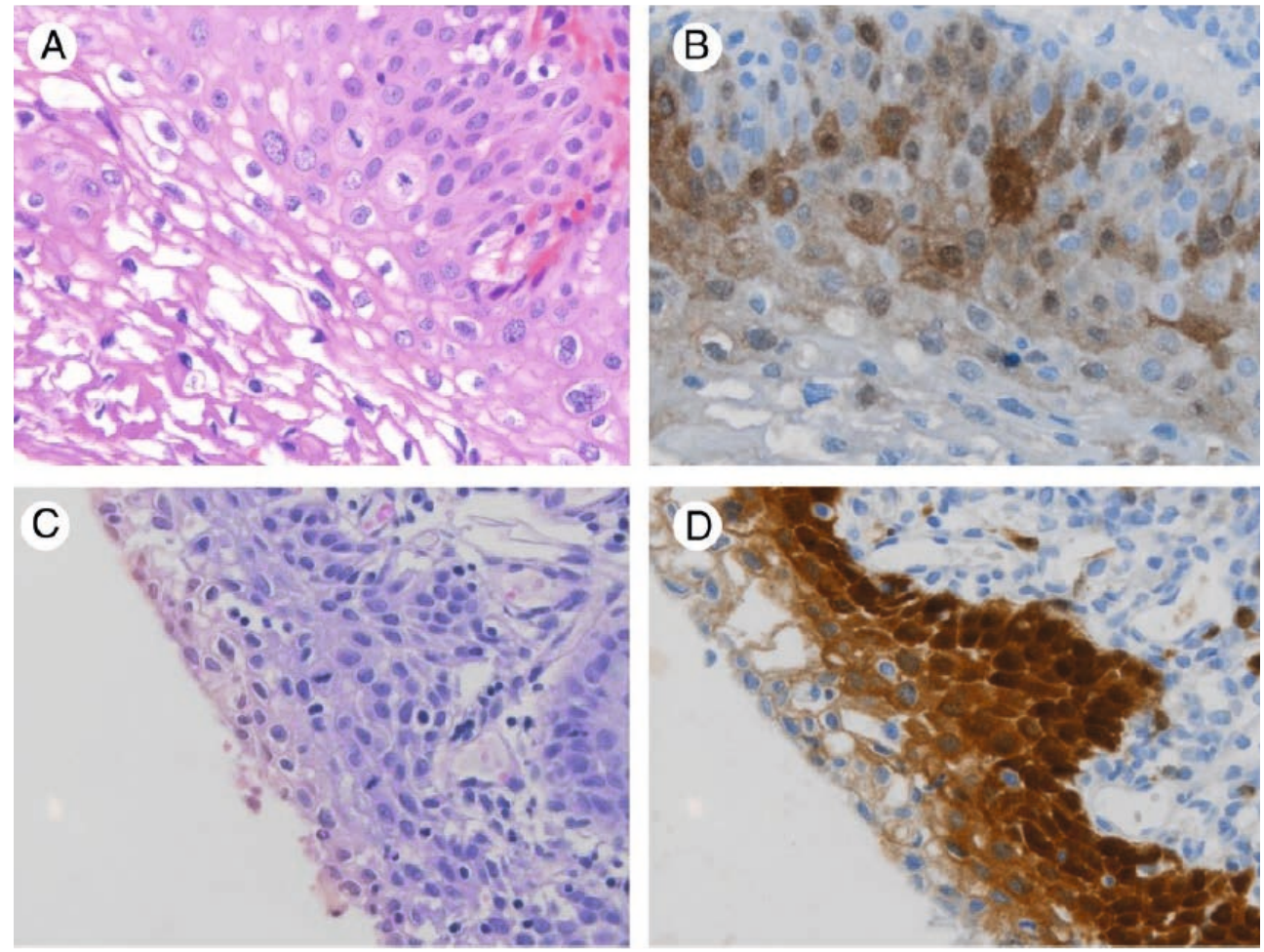

Figure 1. Histological images of CIN2 lesions from study subjects

All images represent a CIN 2 lesion, magnification 40x. A) CIN2 lesion with spontaneous regression during follow-up, HEstaining. B) CIN2 lesion with spontaneous regression during follow-up, p16staining. C) CIN2 lesions with persistence during follow-up, HE staining. D) CIN2 lesion with persistence during follow-up, p16 staining. The proliferating cells extend into the middle of the epithelium. The cells show atypia with nuclear hyperchromasia and increased nuclearcytoplasmic ratio. All cases show overlying koilocytosis. There is patchy expression of 1616 immuno staining in figure $1 B$ and block positive p16 immuno staining of the basal and middle cell layers in figure $1 \mathrm{D}$. 


\section{Potential predictors}

Potential predictors of response were pre-selected based on expert opinions and published studies reporting factors that influence the natural course of $\mathrm{CIN}$, as is advised in methodological guidelines for prognostic modeling. This results in higher external validity than selecting factors that are significantly related to outcome in the same dataset $[11,12]$. Evidence regarding potential predictors of disease regression in $\mathrm{CIN} 2$ is scarce. Moreover, most studies examined immunological and histological biomarkers and not simple clinical parameters [10]. We aimed to develop a widely applicable prediction model, and therefore limited the potential predictors to readily available variables from medical history, such as patient demographics and characteristics of the cervical cytology and biopsy specimens. The final set of potential predictors that was considered for the model included age at diagnosis, smoking, the result of the PAP smear immediately before the $\mathrm{CIN} 2$ diagnosis, the number of individual CIN 2 lesions, and the existence of concomitant $\mathrm{CIN} 1$ and $\mathrm{CIN} 2$ in the same biopsy. Younger age is associated with an increased rate of human papilloma virus (HPV) clearance after treatment [13]. Older age is associated with a higher risk of progression or persistence than younger women and with a higher incidence of residual disease after non-radical surgical excision of high-grade CIN [14, 15]. The severity of pre-treatment cytological abnormalities is associated with residual disease after non-radical surgical treatment of high-grade CIN [14]. Milder cytological abnormalities may indicate that a CIN 2 lesion is lowrisk (more often hr-HPV negative). Indeed, it was recently shown that the risk of cancer in women with a test result of HPV negative abnormal squamous cells of unknown significance (ASCUS) was comparable to that in women with a negative PAP test result [16]. Smoking is associated with an increased risk of cervical cancer, a higher risk of developing high-grade $\mathrm{CIN}$ in patients with abnormal cervical cytology, and disease persistence after surgical treatment [17-19]. The amount of individual CIN 2-lesions was interpreted as comparable to a larger lesion size, which has been associated with non-regression of high-grade CIN [1]. Finally, concomitant CIN 1 and CIN 2 in the same biopsy is a frequent event. Evidence of its prognostic value with regard to disease regression is lacking, but such lesions may be low-risk, with a natural history similar to that of low-grade lesions.

\section{Sample size}

At least ten events should be collected for each potential predictor that is to be evaluated in the multivariable regression analysis [20]. An event is defined as the least frequent outcome status. A recent prospective randomized controlled trial evaluating spontaneous regression of CIN 2 after 24 months showed disease regression in $40 \%$ of patients [21]. In order to develop a model with five potential predictors, at least 50 events would be required, which correlates to a sample size of at least 125 (50/0.4).

\section{Data collection, data quality and missing data}

Patients were recruited from the hospital pathology database. Patient data regarding demography and medical history were extracted from medical records by a trained senior medical student (FL). In case of doubt, data were interpreted by a second person (MK, medical doctor). Data were checked for completeness and inconsistencies (MK). Any inconsistencies or incomplete data were double-checked and corrected or completed if possible. Missing data were imputed using a 
multiple imputation strategy in order to prevent a potentially considerable loss of precision and minimize the probability of introducing bias in the estimation of coefficients [22]. The number of imputations was set to five. A sensitivity analysis was performed by comparing the imputed dataset to the original dataset, in order to determine whether imputation led to significantly different results.

\section{Model development}

For each of the five imputed datasets, all potential predictors were analyzed in a multivariable logistic regression model, using disease regression within 24 months as the outcome variable. Backward stepwise deletion based on the Wald test was applied to reduce the number of predictors in the model, using a $p$-value of 0.20 , as recommended by prediction modeling guidelines [12]. Predictors that remained in at least three out of five imputed datasets were included in the final model. The selected predictors were re-estimated in all imputed datasets separately. The results of the five models were combined into one prediction model.

\section{Internal validation of the model}

Internal validation was performed using the bootstrapping method. This is a method to correct for over-fitting, when the model performs well for the data from which it was developed but provides too extreme predictions for future patients. Backward stepwise deletion can introduce a selection bias, as predictors that are overestimated by chance are more likely to be included than predictors that are underestimated by chance. This may lead to over-fitting. B-bootstrap samples of the same size as the original sample were drawn while replacing the original data, which reflects the drawing of samples from the underlying population. This was repeated 1000 times $(B)$. In this way, the likely performance in future patients could be estimated, and the model was adjusted to make future predictions less extreme.

\section{Performance of the model}

The overall performance of the internally validated model was assessed using Nagelkerke's $R^{2}$ and the Brier score. Nagelkerke's $R^{2}$ is a pseudo $\mathrm{R}^{2}$ compared to the one for linear regression, and can be used to quantify the predictive strength of a model. The higher the Nagelkerke's $R^{2}$, the greater the strength of the model. The Brier score quantifies the average prediction error as the difference between binary outcomes and predictions, and thus should be close to zero. The ability of the model to identify patients in whom a CIN 2 lesion will regress spontaneously was quantified as the area under the receiver operating characteristic curve (AUC). The AUC ranges from $50 \%$ to $100 \%$, indicating no discriminative capacity to perfect discriminative capacity. The agreement between predicted probabilities and observed frequencies of the outcome was assessed by visually inspecting the calibration plot. Last, the Hosmer and Lemeshow $(\mathrm{H}-\mathrm{L})$ goodness-of-fit statistic was computed as a quantitative measure of accuracy. A high outcome of this statistic is related to a low $p$-value, which indicates a poor fit. All statistical analyses were performed using SPSS 22.0 (IBM; Armonk, NY, USA; released 2013) and R 3.2.1 (http://www.r-project.org). 


\section{Results}

\section{Population characteristics}

A total of 129 women were eligible for inclusion in the study. Spontaneous regression within 24 months occurred in 91 of these women (71\%). None of the women showed disease progression beyond CIN 3. Most (106) women completed the 24 month follow-up. Of the 23 women that did not complete the follow-up, those with a test result of PAP 1 after 6 and/or 12 months were included in the regression group $(n=21)$, while the others were included in the non-regression group $(n=2)$. Table 1 shows the population characteristics with regard to the selected predictors in the model.

Table 1. Patient characteristics with regard to the selected predictors in the model

\begin{tabular}{lll}
\hline & Missing (\%) & Total \\
\hline Mean age at diagnosis, mean (range) & 0 & $36(17-74)$ \\
Smoking, no. (\%) & $22(17 \%)$ & $61(47 \%)$ \\
PAP1 or PAP 2 (ASCUS) before CIN 2 diagnosis, no. (\%) & 0 & $21(16 \%)$ \\
Concomitant CIN 1 in same biopsy, no. (\%) & 0 & $46(36 \%)$ \\
More than one biopsy containing CIN 2, no. (\%) & 0 & $9(7 \%)$ \\
\hline
\end{tabular}

\section{Model development and internal validation}

Missing values were only present for smoking as a predictor. After imputation, all 129 women were available for development of the prediction model. After entering the five potential predictor variables in the model, the following predictors met our selection criteria: smoking, a result of PAP 1 or PAP 2 (ASCUS) before the $\mathrm{CIN} 2$ diagnosis, concomitant $\mathrm{CIN}_{1}$ in the same biopsy, and more than one biopsy containing $\mathrm{CIN} 2$. All outcomes are dichotomous variables. These four variables were combined into one model. Table 2 shows the original prediction model that estimates the spontaneous regression of $\mathrm{CIN} 2$ within 24 months. Internal validation of the model by bootstrapping produced a shrinkage factor of 0.95 , which was used to adjust the regression coefficients, as shown in Table 2. To estimate the individual probability of regression of $\mathrm{CIN} 2$ within 24 months, the following predictive equation can be used: $\mathrm{P}\left({ }_{\text {regression }}\right)=100 \% \times 1 /(1+\exp (-$ (0.928 - (0.677 * smoking) + (1.237 * PAP1 or PAP2 before CIN2 diagnosis) + (0.881 * concomitant $\mathrm{CIN} 1$ in same biopsy) - (0.752* more than one biopsy containing $\mathrm{CIN} 2)))$ ). For example, a smoking patient with a pre-biopsy PAP 3 a (cytological LSIL with indications for HPV) and a concomitant $\mathrm{CIN} 1$ in the same biopsy but only one $\mathrm{CIN} 2$ lesion has a predicted probability of regression of $73.7 \%$. With a diagnosis of a pure CIN 2 lesion, the probability of regression would drop to $56.2 \%$. 
Table 2. Prediction model for spontaneous regression of $\mathrm{CIN} 2$

\begin{tabular}{|c|c|c|c|}
\hline Variable & $\begin{array}{l}\text { Regression coefficient } \\
\text { (initial model) }\end{array}$ & $\begin{array}{l}\text { Regression coefficient (inter- } \\
\text { nally validated model) }\end{array}$ & $\begin{array}{l}\text { Odds ratio (initial model) } \\
(95 \% \mathrm{Cl})\end{array}$ \\
\hline Intercept & 0.928 & 0.928 & - \\
\hline Smoking & -0.713 & -0.677 & $0.49(0.18-1.32)$ \\
\hline $\begin{array}{l}\text { PAP } 1 \text { or PAP } 2 \text { before } \\
\text { CIN } 2 \text { diagnosis }\end{array}$ & 1.302 & 1.237 & $3.68(0.78-17.43)$ \\
\hline $\begin{array}{l}\text { Concomitant } \mathrm{CIN} 1 \text { in } \\
\text { same biopsy }\end{array}$ & 0.928 & 0.881 & $2.53(1.05-6.12)$ \\
\hline $\begin{array}{l}\text { More than one bio- } \\
\text { psy containing CIN } 2\end{array}$ & -0.792 & -0.752 & $0.45(0.10-2.06)$ \\
\hline
\end{tabular}

To calculate the absolute risk of regression on CIN 2:

$\mathrm{P}($ regression $)=1 /(1+\mathrm{e}-$ Linear part $) \times 100 \%$.

Linear part $=0.928-\left(0.677^{*}\right.$ smoking $)+(1.237$ *PAP 1 or PAP 2 before $\mathrm{CIN} 2$ diagnosis $)+(0.881$ * concomitant CIN 1 in same biopsy) - (0.752 * more than one biopsy containing CIN 2).

*Regression coefficients after adjustment for over-fitting by shrinkage (shrinkage factor $=0.95$ ).

$\mathrm{Cl}$, confidence interval.

\section{Performance of the model}

The overall performance of the model showed a Nagelkerke's $R^{2}$ of $14 \%$ and a Brier score of 0.19 . Figure 2 shows the ROC curve with the AUC for assessment of the discriminative performance of the prediction model. The AUC was $69.2 \%$ [95\% confidence interval $(\mathrm{Cl}), 58.5-79.9 \%$ ], which indicates a reasonable discriminative ability. A non-significant $\mathrm{H}$ - $\mathrm{L}$ statistic $(0.715$ with $p=0.982)$ indicated good model fit. Figure 3 shows the corresponding calibration curve, representing the accuracy of the model. Predicted probabilities ranged from $38 \%$ to $95 \%$, with a mean of $70.4 \%$ [standard deviation (SD), 14\%]. The developed prediction model has a good fit to the reference curve. The calibration plot shows that the predicted probabilities are especially well calibrated from about $70 \%$ upwards. When using an outcome of $70 \%$ as the cut-off value (with a predicted outcome of $>70 \%$ indicating disease regression and a predicted outcome of $<70 \%$ indicating non-regression), the sensitivity is $73.6 \%$ and the specificity is $55.3 \%$. This represents a positive predictive value of $79.8 \%$ and a negative predictive value of $46.7 \%$ in a population with a similar frequency of outcomes. In this cohort, 84 women had a predicted probability of disease regression $>70 \%$. Of these, 17 patients (20\%) experienced disease persistence. 


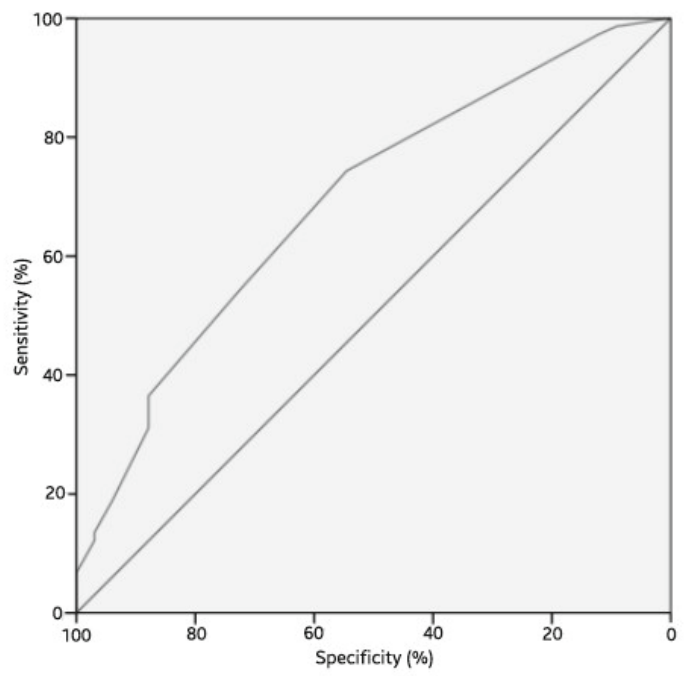

Figure 2. Receiver operating characteristic curve

The AUC of the ROC curve of the prediction model was 69.2\% [95\% confidence interval, 58.5-79.9\%], which indicates a reasonable discriminative ability.

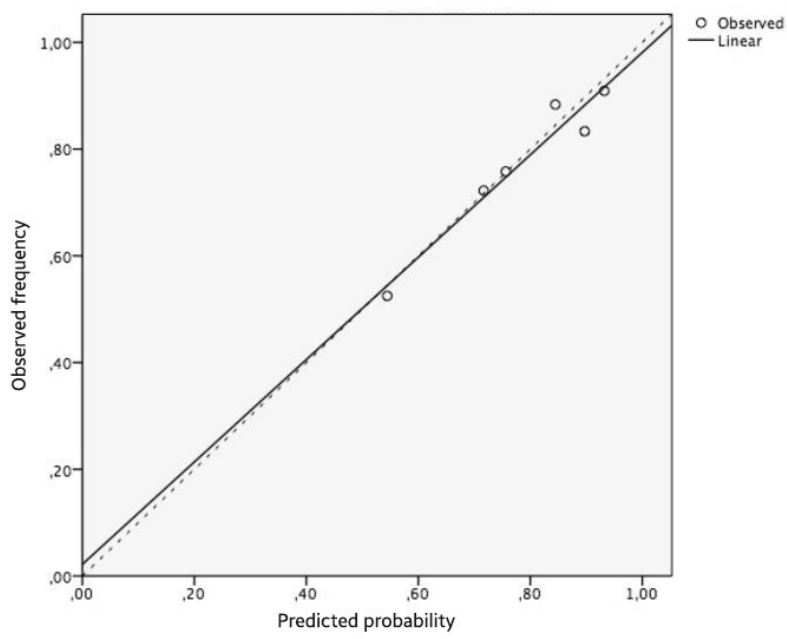

\section{Figure 3. Calibration plot}

The calibration plot indicates the observed frequency of regression by predicted probability (slope, 0.959; intercept, 0.022). The predicted probabilities ranged from $52 \%$ to $95 \%$, with a mean of $70.4 \%$ [SD $14 \%]$. 


\section{Discussion}

This study describes the development and internal validation of a prediction model for spontaneous regression of CIN 2 lesions. The model consists of four simple clinical parameters, including smoking habits, the result of the last PAP smear, concomitant CIN 1 and CIN 2 in one biopsy, and multiple CIN 2 lesions in different biopsies. This prediction model has reasonable discriminative capacity and is accurate, especially in the prediction of disease regression. As such, it can be applied to reassure patients that observational management can be a good option when the model indicates a high probability of regression.

The prediction model was designed for $\mathrm{CIN} 2$, but not $\mathrm{CIN}$ 3, because overtreatment is more common for $\mathrm{CIN} 2$ than for $\mathrm{CIN} 3$. This is due to the fact that $\mathrm{CIN} 2$ has a higher spontaneous regression rate than $\mathrm{CIN}_{3}[5]$. Indeed, international guidelines also advocate conservative treatment of younger women with $\mathrm{CIN} 2 .[7,8]$ In previous studies on the conservative treatment of $\mathrm{CIN} 2$, no women progressed beyond $\mathrm{CIN} 3$ within 12 months $[6,23,24]$. This was confirmed in our study, which showed no disease progression beyond CIN 3 within 24 months of follow-up. These findings indicate that observational management of $\mathrm{CIN} 2$ with adequate follow-up is safe and may prevent overtreatment. It must be noted, however, that discussion has evolved as to whether $\mathrm{CIN} 2$ and $\mathrm{CIN} 3$ are truly two grades of the same pathological condition. Additional studies on epigenetic changes may subdivide CIN lesions into early or advanced lesions, carrying a lower or higher risk of malignant transformation, regardless of the conventional histopathological diagnosis.[25] However, such tests are currently not available in clinical practice. Since the differentiation between $\mathrm{CIN} 2$ and $\mathrm{CIN} 3$ is still based on histopathology, this study focused on CIN 2 because of its high spontaneous regression rate and the safety of conservative management.

The prediction model aims at widespread applicability by using simple clinical parameters as predictors. Two previous models were developed to predict the risk of progression and regression of CIN 1-2 and CIN 2-3 lesions, but included several biomarkers for which additional immunohistochemical staining was necessary. $[1,26]$ Both models have good predictive values, but utilize predictors that are not readily available and require additional tests. This reduces the clinical applicability of these models. For the same reason, HPV genotyping was not included in our model as a predictor. Indeed, the presence of high-risk HPV types in CIN 2 lesions are strongly predictive for non-regression, but HPV genotyping is not routinely performed at all medical institutions.[2, 27, 28] Although most countries and institutions will apply HPV-based cervical cancer screening in the future, cervical cytology will be used in clinical practice outside screening programs and in those institutions where HPV-based cervical cancer screening cannot be applied. Our prediction model is based entirely on simple clinical parameters and is consequently widely applicable in different patient populations.

Interestingly, age was dropped from the prediction model during backward stepwise deletion. As mentioned before, previous research suggests an increased probability of disease progression of LSIL with increasing age and that HPV clearance is more common in younger patients. In our cohort, age did not influence the outcome (data not shown). Although age is not part of 
the prediction model, it seems sensible to take age into account when counseling a patient for conservative treatment. The largest benefit of conservative treatment will be for younger patients with a pregnancy wish in the future, by omitting surgical treatment and the associated risk of preterm birth. As such, conservative treatment is primarily advised for younger patients. The current prediction model aids the decision-making in these patients based on four other parameters.

The discriminative ability of the prediction model can be considered reasonable-to-good. The AUC can range from 50 to $100 \%$, indicating no discriminative capacity to perfect discriminative capacity. Generally, an AUC of $70 \%$ and higher is considered indicative of good discriminative ability. The AUC of our model is $69.2 \%$, which almost reaches $70 \%$ and therefore indicates a reasonable-to-good discriminative ability. The model is most suitable for prediction of disease regression in patients with high predicted probabilities of disease regression. Although a cutoff value of $70 \%$ identifies $80 \%$ of patients who will show disease regression correctly, the $20 \%$ of patients will still experience disease persistence and could be considered 'under treated'. However, several studies have shown that disease progression within the follow up period of our study is very rare, making watchful waiting a safe option for all patients. [6, 23, 24] We therefore advocate the use of this model to reassure patients that observation is an appropriate treatment option. As such, the model could function as a tool for a more individualized approach to $\mathrm{CIN} 2$ management.

Strengths of the study include the population size and robust statistical methods. Another strength of the study is the internal validation procedure, as an alternative to external validation when a validation population is lacking, Large populations of untreated women with $\mathrm{CIN} 2$ are currently scarce, as conservative management of CIN 2 has only recently been included in treatment guidelines. In these situations, it is not uncommon to apply only internal validation to a newly developed prediction model $[29,30]$. Prospective external validation is of course desirable and encouraged. An important limitation to this study is the low reproducibility of $\mathrm{CIN} 2$ diagnoses [31]. All biopsies were assessed according to the WHO guidelines. The biopsies were not re-analyzed for this study, as this does not necessarily lead to a more reliable diagnosis and is not performed in routine clinical practice. Another potential limitation of the study is the high observed regression rate in our population (71\%). The sample size calculation in this study was based on the regression rate of $\mathrm{CIN} 2$ in a randomized controlled trial. However, studies on spontaneous regression of CIN 2 show a wide range of regression rates over different follow-up periods, with several studies reporting high regression rates of up to $71 \%[32,33]$. Our high regression rate may therefore be the upper range of normal, or may be (partially) due to a selection bias, as a wait-and-see policy may have been largely applied to patients with a high perceived chance of disease regression. Another explanation could be that some patients were over-diagnosed, as a result of the low reproducibility of $\mathrm{CIN} 2$ diagnoses. Prospective validation of the model should clarify this issue. Additionally, a prospective study should assess the usefulness and clinical impact of the model, in order to assess whether its implementation leads to the desired effect of less overtreatment of CIN 2, without under-treatment. 


\section{Conclusion}

We developed and internally validated a prediction model for spontaneous regression of CIN 2 lesions in a Western population. Upon external validation of its discriminatory capacity and accuracy, the model could be used in clinical practice to counsel patients with a CIN 2 diagnosis. When there is a high predicted chance of disease regression, the model could be used to reassure the patient that observation is an appropriate treatment option. As such, the model could function as a tool for a more individualized approach to CIN 2 management, and its use could reduce unnecessary treatment of $\mathrm{CIN} 2$ lesions and the associated complications. 


\section{References}

1. MunkAC, Gudlaugsson E, Ovestad IT, etal. Interaction of epithelial biomarkers, local immune response and condom use in cervical intraepithelial neoplasia 2-3 regression. Gynecol Oncol. 2012 Dec;127(3):489-94.

2. Trimble CL, Piantadosi S, Gravitt $\mathrm{P}$, et al. Spontaneous regression of high-grade cervical dysplasia: effects of human papillomavirus type and HLA phenotype. Clin Cancer Res. 2005 Jul 1;11(13):4717-23.

3. Crane JM. Pregnancy outcome after loop electrosurgical excision procedure: a systematic review. Obstet Gynecol. 2003 Nov;102(5 Pt 1):1058-62.

4. Kyrgiou M, Koliopoulos G, Martin-Hirsch P, Arbyn M, Prendiville W, Paraskevaidis E. Obstetric outcomes after conservative treatment for intraepithelial or early invasive cervical lesions: systematic review and meta-analysis. Lancet. 2006 Feb 11;367(9509):489-98.

5. Holowaty P, Miller AB, Rohan T, To T. Natural history of dysplasia of the uterine cervix.] N Natl Cancer Inst. 1999 Feb 3;91(3):252-8.

6. McAllum B, Sykes PH, Sadler L, Macnab H, Simcock B], Mekhail AK. Is the treatment of CIN 2 always necessary in women under 25 years old? Am J Obstet Gynecol. 2011 Nov;205(5):478 e1-7.

7. Committee on Adolescent Health C. ACOG Committee Opinion No. 436: evaluation and management of abnormal cervical cytology and histology in adolescents. Obstet Gynecol. 2009 Jun;113(6):1422-5.

8. Massad LS, Einstein MH, Huh WK, et al. 2012 updated consensus guidelines for the management of abnormal cervical cancer screening tests and cancer precursors.] Low Cenit Tract Dis. 2013 Apr;17(5 Suppl 1):S1-S27.

9. Kinney W, Hunt WC, Dinkelspiel H, et al. Cervical excisional treatment of young women: a population-based study. Gynecol Oncol. 2014 Mar;132(3):628-35.

10. Koeneman MM, Kruitwagen RF, Nijman HW, Slangen BF, Van Corp T, Kruse A]. Natural history of high-grade cervical intraepithelial neoplasia: a review of prognostic biomarkers. Expert Rev Mol Diagn. 2015 Apr;15(4):527-46.

11. Steyerberg EW, Moons KG, van der Windt DA, et al. Prognosis Research Strategy (PROGRESS) 3: prognostic model research. PLoS Med. 2013;10(2):e1001381.

12. Harell F]. Regression Modeling Strategies. New York: NY: Springer; 2001.

13. Rositch AF, Soeters HM, Offutt-Powell TN, Wheeler BS, Taylor SM, Smith JS. The incidence of human papillomavirus infection following treatment for cervical neoplasia: a systematic review. Gynecol Oncol. 2014 Mar;132(3):767-79.

14. Quint KD, de Koning MN, Quint WG, Pirog EC. Progression of cervical low grade squamous intraepithelial lesions: in search of prognostic biomarkers. Eur ] Obstet Cynecol Reprod Biol. 2013 Oct;170(2):501-6.

15. Fu Y, Chen C, Feng S, et al. Residual disease and risk factors in patients with high-grade cervical intraepithelial neoplasia and positive margins after initial conization. Ther Clin Risk Manag. 2015;11:851-6. 
16. Gage JC, Katki HA, Schiffman M, et al. The low risk of precancer after a screening result of human papillomavirus-negative/atypical squamous cells of undetermined significance papanicolaou and implications for clinical management. Cancer Cytopathol. 2014 Nov;122(11):842-50.

17. Guarisi R, Sarian LO, Hammes LS, et al. Smoking worsens the prognosis of mild abnormalities in cervical cytology. Acta Obstet Gynecol Scand. 2009;88(5):514-20.

18. de Mello Silva MV, Coutinho IC, de Andrade Heraclio S, Fittipaldi HM, Jr., Katz L. Factors associated with the persistence/recurrence of $\mathrm{CIN} 2 / 3$ in women submitted to loop electrosurgical excision procedure in a teaching hospital in northeastern Brazil: a casecontrol study. J Low Genit Tract Dis. 2014 Oct;18(4):286-90.

19. Cadducci A, Barsotti C, Cosio S, Domenici L, Riccardo Cenazzani A. Smoking habit, immune suppression, oral contraceptive use, and hormone replacement therapy use and cervical carcinogenesis: a review of the literature. Gynecol Endocrinol. 2011 Aug;27(8):597-604.

20. Peduzzi P, Concato ], Kemper E, Holford TR, Feinstein AR. A simulation study of the number of events per variable in logistic regression analysis. J Clin Epidemiol.1996 Dec;49(12):1373-9.

21. Castle PE, Schiffman M, Wheeler CM, Solomon D. Evidence for frequent regression of cervical intraepithelial neoplasia-grade 2. Obstet Gynecol. 2009 Jan;113(1):18-25.

22. Donders AR, van der Heijden G], Stijnen T, Moons KG. Review: a gentle introduction to imputation of missing values.] J Clin Epidemiol. 2006 Oct;59(10):1087-91.23. Ho GY, Einstein MH, Romney SL, et al. Risk factors for persistent cervical intraepithelial neoplasia grades 1 and 2: managed by watchful waiting. J Low Cenit Tract Dis. 2011 Oct;15(4):268-75.

24. Discacciati MG, de Souza CA, d'Otavianno MG, et al. Outcome of expectant management of cervical intraepithelial neoplasia grade 2 in women followed for 12 months. Eur ] Obstet Gynecol Reprod Biol. 2011 Apr;155(2):204-8.

25. Steenbergen RD, Snijders P], Heideman DA, Meijer C]. Clinical implications of (epi)genetic changes in HPV-induced cervical precancerous lesions. Nat Rev Cancer. 2014 Jun;14(6):395405.

26. Kruse A], Skaland I, Janssen EA, et al. Quantitative molecular parameters to identify low-risk and high-risk early CIN lesions: role of markers of proliferative activity and differentiation and Rb availability. Int ] Cynecol Pathol. 2004 Apr;23(2):100-9.

27. Ovestad IT, Gudlaugsson E, Skaland I, et al. The impact of epithelial biomarkers, local immune response and human papillomavirus genotype in the regression of cervical intraepithelial neoplasia grades 2-3. J Clin Pathol. 2011 Apr;64(4):303-7.

28. Schiffman M, Boyle S, Raine-Bennett T, et al. The Role of Human Papillomavirus Cenotyping in Cervical Cancer Screening: A Large-Scale Evaluation of the cobas HPV Test. Cancer Epidemiol Biomarkers Prev. 2015 Sep;24(9):1304-10.

29. Schoorel EN, van Kuijk SM, Melman S, et al. Vaginal birth after a caesarean section: the development of a Western European population-based prediction model for deliveries at term. BJOG. 2014 Jan;121(2):194-201

30. Vergeldt TF, van Kuijk SM, Notten KJ, Kluivers KB, Weemhoff M. Anatomical Cystocele Recurrence: Development and Internal Validation of a Prediction Model. Obstet Cynecol. 2016 Feb;127(2):341-7.

31. Carreon JD, Sherman ME, Guillen D, et al. CIN2 is a much less reproducible and less valid 
diagnosis than CIN3: results from a histological review of population-based cervical samples. Int ] Gynecol Pathol. 2007 Oct;26(4):441-6.

32. Monteiro DL, Trajano A], Russomano FB, Silva KS. Prognosis of intraepithelial cervical lesion during adolescence in up to two years of follow-up. J Pediatr Adolesc Cynecol. 2010 Aug;23(4):230-6.

33. Munro A, Powell RG, Cohen P, et al. Spontaneous regression of $\mathrm{CIN} 2$ in women aged 18-24 years: a retrospective study of a state-wide population in Western Australia. Acta Obstet Gynecol Scand. 2016 Mar; 95(3):291-8 


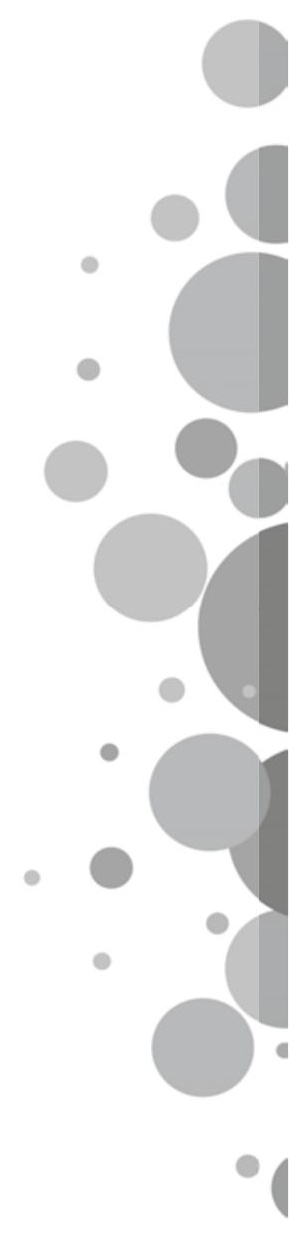




\section{CHAPTER 6}

Smoking status and parity are associated with spontaneous regression of high-risk HPV-positive CIN2

Margot M Koeneman, Natasja Hendriks, Loes FS Kooreman, Bjorn Winkens, Roy FPM Kruitwagen, Arnold ] Kruse

Submitted 


\section{Abstract}

Background: The majority of Cervical Intraepithelial Neoplasia (CIN) 2 lesions will regress without treatment. Conservative management is often advised, but the natural history of an individual lesion remains unpredictable. Identification of prognostic factors in $\mathrm{CIN} 2$ lesions would enable a more individualized patient counseling with regard to management options. Since the implementation of HPV-based screening for cervical cancer, the majority of $\mathrm{CIN} 2$ lesions is highrisk (hr)HPV positive. Evidence on prognostic factors in hrHPV-positive CIN2 is lacking.

Objective: The aim of this study is to identify prognostic factors for the spontaneous regression of exclusively high-risk HPV positive CIN2.

Study design: This registration-based retrospective cohort study was conducted at the Maastricht University Medical Center, Maastricht, the Netherlands. Women with a hrHPV positive CIN2 lesion who received observational management (including cytological follow-up) between January 1, 2000, and April 30, 2013 were included. Regression was defined as PAP $1 / 2$ cytology or $\leq \mathrm{CIN} 1$ histology at the 24-month follow-up and no diagnosis of $\geq \mathrm{CIN} 2$ before the 24-month follow-up visit. Potential prognostic factors (HPV16/18, p16 staining, KI67 staining, age, smoking status, last PAP smear result, multiple $\mathrm{CIN} 2$ lesions, oral contraception use and parity) were assessed using logistic regression analysis.

Results: 56 women were included in the study, of which 34 (61\%) showed spontaneous regression of their lesion. Not smoking and nulliparity were identified as significant prognostic factors for disease regression (OR 3.84 [1.04-14.21] and OR 5.00 [1.32-19.00], respectively).

Conclusions: Smoking status and parity influence the likelihood of disease regression in hrHPV positive $\mathrm{CIN} 2$. These factors should be considered in individual patient counseling regarding the choice between immediate treatment or conservative management. 


\section{Introduction}

High-grade cervical intraepithelial neoplasia (CIN 2-3) is caused by cervical infection with Human Papillomavirus (HPV) and is the precursor of cervical carcinoma. However, not all CIN lesions progress to cervical cancer. A significant subset of lesions regresses spontaneously without treatment. $[1,2]$ This is especially true for $\mathrm{CIN} 2$ : studies have shown a spontaneous regression rate of up to $74 \%$ after 12 months follow-up.[3-6]

Most high-grade lesions, including $\mathrm{CIN} 2$, are treated by surgical excision, which is most commonly performed by large loop excision of the transformation zone (LLETZ). Given the rate of spontaneous regression, this leads to significant overtreatment. This may result in unnecessary side effects, such as continued bleeding, vaginal discharge and more importantly, a risk of premature birth in subsequent pregnancies. [7, 8] For this reason, international guidelines now advice upon conservative management of younger women with $\mathrm{CIN}$ 2.[9]

Ideally, the natural prognosis of an individual lesion would be predictable, to allow for selection of those women in whom spontaneous regression is to be expected. This would enable a more individualized management of $\mathrm{CIN} 2$. To this aim, several studies aimed to identify prognostic (bio) markers or prediction models for the natural history of CIN lesions. However, most studies were performed in mixed populations of $\mathrm{CIN} 1 / 2$ and $\mathrm{CIN}_{2} / 3$ lesions and not exclusively $\mathrm{CIN} 2$ lesions. [6, 10-13] Those studies that did evaluate prognostic markers in exclusively CIN2, included both HPV positive and negative lesions.[4, 14-16] Since most screening programs for cervical cancer have recently switched to an HPV based approach, the majority of newly diagnosed CIN2 lesions will be high-risk (hr)HPV positive. As hrHPV in itself is considered to be a prognostic marker in $\mathrm{CIN}$, this may alter the effect of other predictive markers on disease outcome in a population that is exclusively hrHPV positive.

Evidence on prognostic markers in exclusively hrHPV positive $\mathrm{CIN} 2$ is lacking, which hampers adequate counseling of patients with such lesions. For this reason, we designed the current study, which aims to identify prognostic factors for disease regression in hrHPV positive CIN2.

\section{Materials and methods}

\section{Setting}

This registration-based retrospective cohort study was conducted at the Maastricht University Medical Center, Maastricht, the Netherlands. Approval for this study was obtained from the Medical Ethics Committee of Maastricht University Medical Center (approval number: METC 154-174; September 2, 2015).

\section{Population}

Patients were extracted from an existing database of a previous study by the authors.[17] In the previous study, patients were included with a biopsy proven $\mathrm{CIN} 2$ lesion who received observational management between January 1, 2000, and April 30, 2013. For the current study, 
we selected from this database all patients with a pure CIN2 diagnosis, who were hrHPV positive. Observational management of CIN 2 consisted of serial Papanicolaou (PAP) tests after 6, 12, and 24 months. A new colposcopy was planned in case of PAP $3 a 2$ (cytological high-grade squamous intraepithelial lesion) or higher at either of the follow-up visits.

\section{Pathological assessment}

All baseline biopsies were intitially assessed by a gynecopathologist in routine diagnostics. After case selection all biopsies were reassessed by a second gynecopathologist, with the aim to select those lesions with exclusively $\mathrm{CIN} 2$ (and not $\mathrm{CIN}_{1}-2$ ). In case of discrepancies with the primary routine diagnosis, a third gynecopathologist reviewed the case, after which a consensus diagnosis was made between the second and third gynecopathologist. The histopathological assessment of cervical biopsies was performed according to national guidelines, based on the World Health Organization criteria, and was based on hematoxylin and eosin staining (figure 1), with additional p16 and KI67 staining. p16 (DAKO clone MX007) and KI67 (DAKO clone MIB1) staining were done via DAKO Autostainer. Strong P16 positivity was determined by strong and diffuse en block staining (figure 2). Weak p16 staining was anything other than negativity. KI67 expression was considered low (non or sporadic expression of cells) or high (more than sporadic, figure 3).

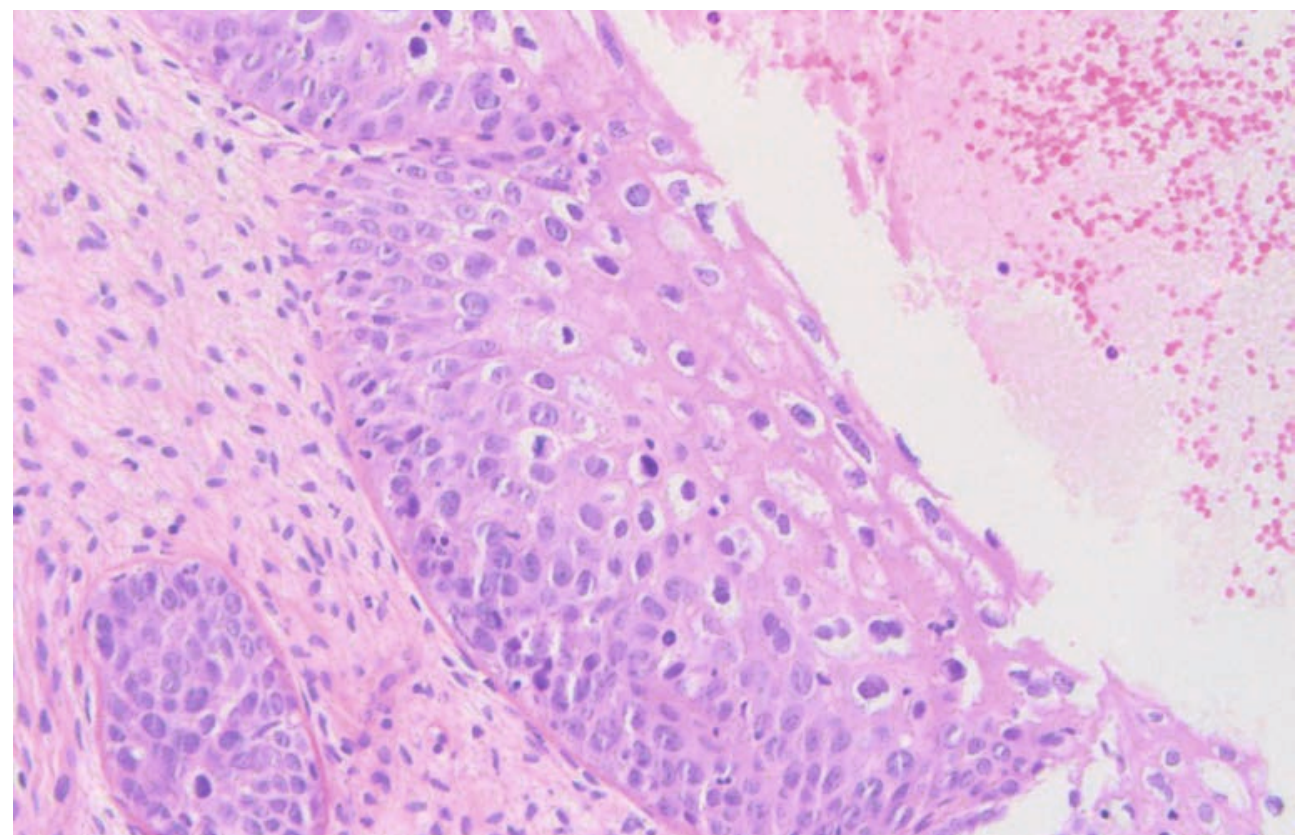

\section{Figure 1: HE staining}

Haematoxylin eosin stained FFPEslide ofCIN2 lesion. The lesion shows basal and suprabasal atypia and mitotic activity of the squamous epithelium with a koilocytic overlay. 


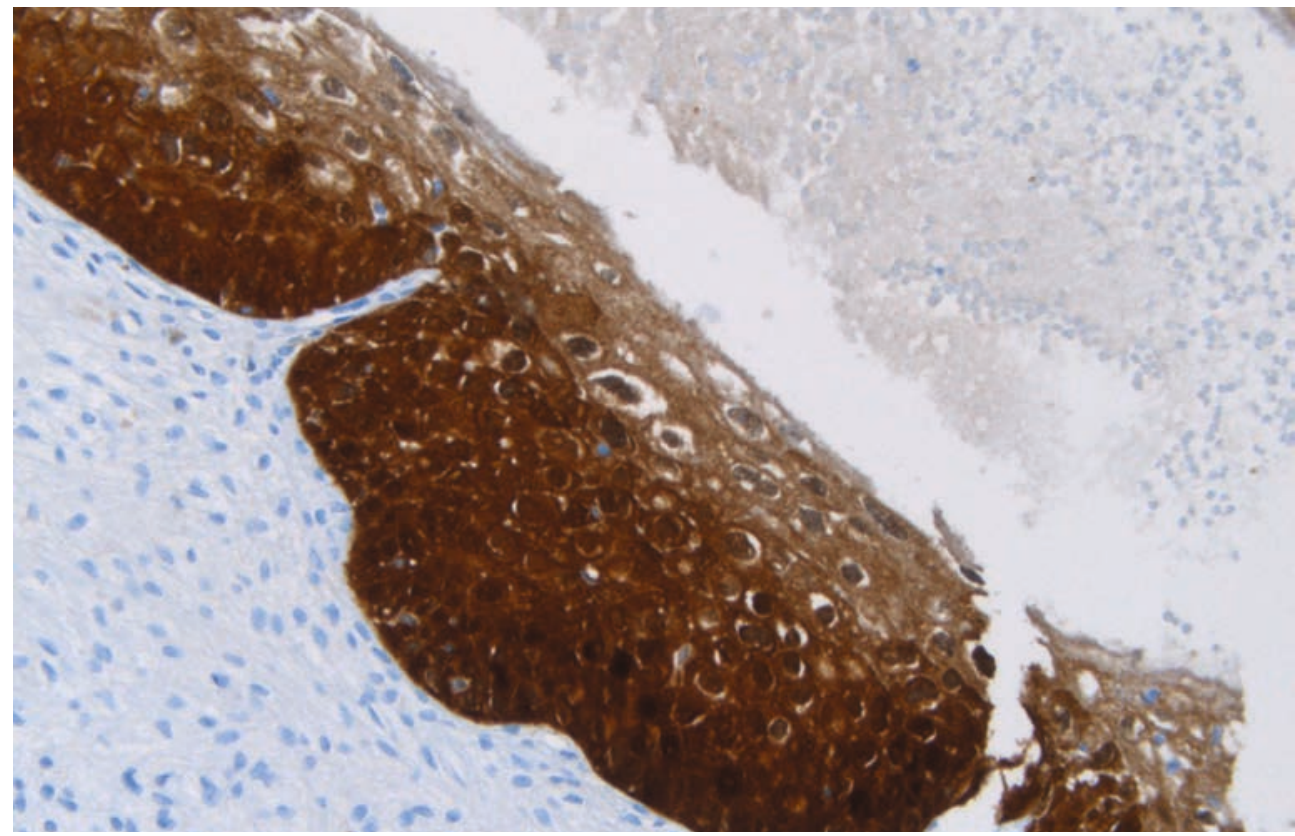

\section{Figure 2: en block p16 positivity}

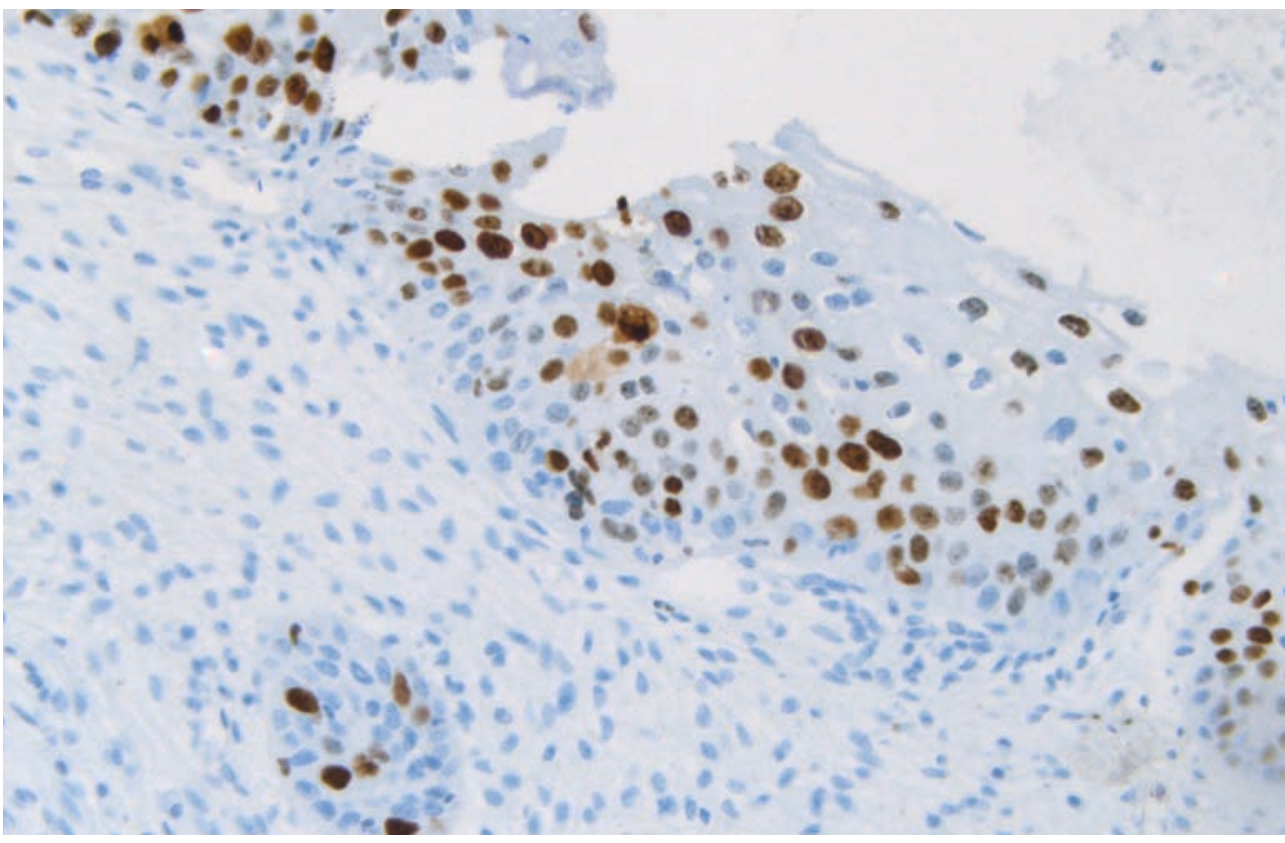

Figure 3: high KI67 expression. 


\section{HPV typing}

Annotations of the affected epithelium were made on the HE slide, after which DNA isolation of the formaline fixed paraffin-embedded tissue was performed via Maxwell 16 (Promega). A PCR GP5+/6+ was run with HPV universal primers. HPV positivity was assessed via agarose gel electrophoresis. Positive HPV cases were subtyped using an Enzym Immuno Assay. Possible outcomes are positivity for HPV16, HPV18 or cocktail hrHPV $(31,33,35,39,45,51,52,56,58,59,66)$.

\section{Outcome measures}

Disease regression was defined as follows. For patients who completed the follow-up schedule, disease regression was defined PAP $1 / 2$ cytology or $\leq \mathrm{CIN} 1$ histology at the 24-month follow-up and no diagnosis of $\mathrm{CIN} 2$ or higher before the 24-month follow-up visit. For patients who did not complete the 24-month follow-up schedule, those with PAP $1 / 2$ cytology at the last follow-up visit were included in the regression group, and all other patients were included in the persistence group.

Potential prognostic factors were selected based on expert opinion and published studies reporting factors that influence the natural course of $\mathrm{CIN}$, taking into account the availability of these factors in the studied population. The following potential predictors were assessed: (1) HPV16/18 negative lesion in baseline biopsy, (2) weak p16 staining in baseline biopsy, (3) weak $\mathrm{KI} 67$ staining in baseline biopsy, (4) lower age at CIN2 diagnosis, (5) not smoking at time of CIN2 diagnosis, (6) last PAP smear result before $\mathrm{CIN}_{2}$ diagnosis < PAP 3 (i.e. PAP1 or PAP 2), (7), no more than one $\mathrm{CIN} 2$ lesion in case of multiple baseline biopsies, (8) no use of oral contraception at time of diagnosis and (9) nulliparity at time of diagnosis.

\section{Statistical analysis}

Statistical analysis was performed using IBM SPSS Statistics for windows (version 24.0. Armonk, NY: IBM Corp). Baseline characteristics were summarized using mean (standard deviation (SD), range) for numerical variables, and number of patients (\%) for categorical variables. The influence of potential prognostic factors on spontaneous regression was first assessed using univariable logistic regression. Additionally, multivariable logistic regression analysis was performed, correcting for age and HPV 16/18 status. For each prognostic variable, the correction was performed in two separate analyses, as correction for both potential confounders was considered inappropriate given the small sample size. Outcomes were presented as odds ratios (OR) with $95 \%$ confidence intervals $(\mathrm{Cl})$. In case of missing data, these cases were omitted from the analysis (complete case analysis, CCA). A two-sided p-value smaller than or equal to 0.05 was considered statistically significant.

\section{Results}

\section{Population}

81 cases of $\mathrm{CIN} 2$ were identified and included in the study. After revision, 23 cases were excluded (8 cases were considered $\mathrm{CIN}_{1}$ or $\mathrm{CIN} 1-2$ and 15 cases were considered $\mathrm{CIN} 3$ ). Of the remaining $58 \mathrm{CIN} 2$ lesions, 56 were positive for hrHPV and were included in the current study. Patient characteristics can be found in table 1 . 
Table 1. Patient characteristics

\begin{tabular}{lll}
\hline Predictor & Missing values & Outcome \\
\hline Age (mean, SD, range) & - & $37(9,17-61)$ \\
Complete follow-up $(\mathrm{n}, \%)$ & - & $45(80 \%)$ \\
Spontaneous regression $(\mathrm{n}, \%)$ & - & $34(61 \%)$ \\
HPV 16/18 negative $(\mathrm{n}, \%)$ & - & $38(68 \%)$ \\
Weak p16 staining (n, \%) & - & $36(64 \%)$ \\
Weak KI67 staining (n, \%) & - & $14(25 \%)$ \\
Not smoking at time of diagnosis (n, \%) & $13(23 \%)$ & $21(49 \%)$ \\
Last PAP smear result before CIN2 diagnosis <PAP 3 (n, \%) & - & $5(9 \%)$ \\
No more than one CIN2 lesion in case of multiple biopsies $(\mathrm{n}, \%)$ & - & $51(91 \%)$ \\
No use of oral contraception at time of diagnosis (n, \%) & $7(13 \%)$ & $27(55 \%)$ \\
Nulliparity $(\mathrm{n}, \%)$ & $9(16 \%)$ & $20(43 \%)$ \\
\hline
\end{tabular}

\section{Prognostic factors for spontaneous regression}

The results of the logistic regression analysis are presented in table 2. Of all studied potential prognostic factors, only negative smoking status and nulliparity were significantly associated with disease regression (OR 3.84 [1.04-14.21] and 5.00 [1.32-19.00], respectively, in the univariable analysis). Both effects remained after correction for age and HPV16/18 in a multivariable regression analysis. With regard to age, similar conclusions were obtained if age was dichotomised (age $<=$ 25 or age $<=30$ ). In smoking women, disease regression occurred in 10 out of 22 women (46\%), compared to 16 out of 21 non-smoking women ( $76 \%$ ). In parous women, regression occurred in 12 out of 27 women (44\%), compared to 16 out of 20 nulliparous women (80\%). Nulliparous women who did not smoke $(n=13)$ showed disease regression in 11 cases ( $85 \%)$. Smoking, parous women $(\mathrm{n}=15)$ showed disease regression in five cases $(33 \%)$. Logistic regression analysis could not be applied to the factor 'last PAP smear result', as there were only five women with a result $<$ PAP3, all of which showed spontaneous regression. Fisher's exact test was applied and showed a $p$-value of 0.143 . 
Table 2. Results of logistic regression analysis of potential predictors for spontaneous regression of hrHPV positive CIN2

\begin{tabular}{|c|c|c|c|c|c|c|}
\hline \multirow[b]{2}{*}{ Predictor } & \multicolumn{2}{|c|}{ Univariable analysis } & \multicolumn{2}{|c|}{$\begin{array}{l}\text { Multivariable analysis, } \\
\text { correction for age }\end{array}$} & \multicolumn{2}{|c|}{$\begin{array}{l}\text { Multivariable analysis, } \\
\text { correction for HPV 16/18 } \\
\text { negative }\end{array}$} \\
\hline & OR $[95 \% \mathrm{Cl}]$ & p value & OR $[95 \% \mathrm{Cl}]$ & pvalue & OR $[95 \% \mathrm{Cl}]$ & p value \\
\hline Age & $1.00[0.95-1.06]$ & 0.95 & - & - & $1.03[0.32-3.24]$ & 0.95 \\
\hline HPV $16 / 18$ negative & $0.98[0.31-3.08]$ & 0.97 & $0.97[0.31-3.09]$ & 0.96 & - & - \\
\hline Weak p16 staining & $1.05[0.34-3.20]$ & 0.94 & $1.05[0.34-3.20]$ & 0.94 & $1.05[0.34-3.21]$ & 0.93 \\
\hline Weak KI67 staining & $0.82[0.24-2.80]$ & 0.75 & $0.78[0.21-2.96]$ & 0.71 & $0.81[0.23-2.85]$ & 0.74 \\
\hline $\begin{array}{l}\text { Not smoking at } \\
\text { time of diagnosis }\end{array}$ & $3.84[1.04-14.21]$ & $0.04 *$ & $3.84[1.04-14.24]$ & $0.04 \%$ & $3.84[1.03-14.33]$ & $0.04^{*}$ \\
\hline $\begin{array}{l}\text { No more than one } \\
\mathrm{CIN} 2 \text { lesion }\end{array}$ & $2.53[0.39-16.51]$ & 0.33 & $2.61[0.38-17.85]$ & 0.33 & $2.64[0.39-17.95]$ & 0.32 \\
\hline $\begin{array}{l}\text { No use of oral con- } \\
\text { traception at time } \\
\text { of diagnosis }\end{array}$ & $0.50[0.16-1.62]$ & 0.25 & $0.49[0.15-1.60]$ & 0.24 & $0.50[0.15-1.63]$ & 0.25 \\
\hline $\begin{array}{l}\text { Nulliparity at time } \\
\text { of diagnosis }\end{array}$ & $5.00[1.32-19.00]$ & $0.02 *$ & $9.82[1.75-55.04]$ & $0.01^{*}$ & $5.00[1.32-19.00]$ & $0.02 *$ \\
\hline
\end{tabular}

*significant

\section{Discussion}

In this study, we identified smoking status and nulliparity as prognostic factors in the natural history of hrHPV positive CIN2. No significant effect was found for HPV16/18, p16 staining, KI67 staining, age, last PAP smear result, multiple CIN2 lesions and oral contraception as potential prognostic factors.

To the best of our knowledge, this is the first study to assess potential predictors of spontaneous regression in exclusively hrHPV positive $\mathrm{CIN} 2$ lesions. Previous studies that assessed prognostic markers in exclusively $\mathrm{CIN} 2$ lesions included either a mixed population of both hrHPV positive and negative women or a population with unknown HPV status.[4, 14-16] We hypothesized that the effect of potential predictors may be different in a population of exclusively high-grade HPV positive $\mathrm{CIN} 2$, due to a prognostic effect of hrHPV itself. Indeed, hrHPV genotypes were found to predict an increased disease progression and a decreased disease regression in several studies in high-grade CIN lesions.[13] As such, a population of exclusively hrHPV positive CIN2 lesions reflects a more 'high-risk' population than a population that also includes hrHPV negative lesions, which may influence potential prognostic factors in the two populations. Indeed, our study results were different from previous studies in $\mathrm{CIN} 2$ lesions. The previous studies on prognostic factors in hrHPV positive and negative $\mathrm{CIN} 2$ lesions identified various prognostic markers, although findings were not always consistent. Non-consistent results among the different studies were found for age, lesions size, oral contraception use and p-16 staining.[4, 14-16] Furthermore sexarche and parity were each evaluated in one study and were both found 
not to be significantly prognostic for spontaneous regression. [4, 15] Ki67 staining was found to be predictive in one study.[15] Smoking status was evaluated in two studies and was negatively correlated with disease regression in both studies. $[14,15]$ In our study of patients with exclusively hrHPV positive CIN2 lesions, we only identified smoking status and parity as prognostic factors. We found no prognostic effect of the other studied factors. This may indeed be explained by the fact that all lesions were hrHPV positive, reflecting a more 'high-risk' population, in which previously determined prognostic factors are of less influence on the natural history of the lesion. This theory is supported by the fact that the regression rate in the current study is lower than in the previous study in the same cohort (61\% vs 71\%).[17] It cannot be excluded, however, that the lack of prognostic factors is the result of a relatively small patient population. Nevertheless, the findings of our study indicate that conventional prognostic factors may not be of equal importance in a population with exclusively hrHPV positive $\mathrm{CIN} 2$ lesions, as compared to populations with both hrHPV positive and negative CIN2 lesions. The relative lack of prognostic factors in hrHPV positive $\mathrm{CIN} 2$ hampers individual patient counseling. Future research should therefore also focus on other potential prognostic factors, among which may be molecular markers of oncogenic processes.

Although hrHPV positivity may diminish the effect of potential prognostic biomarkers in $\mathrm{CIN} 2$, hrHPV genotypes were not found to be prognostic of disease regression in the two studies in $\mathrm{CIN}_{2}$ lesions that assessed this potential biomarker. $[15,16]$ This is in contrast to previous studies in high-grade $\mathrm{CIN}$ lesions that quite consistently identified hrHPV genotypes as prognostic factors for disease progression or non-regression. [13] With regard to exclusively CIN2 lesions, Miyamoto et al. retrospectively included 112 women, of which 28 (25\%) showed disease regression. [15] HPV genotyping was performed in only 40 women and nearly all were positive for high-risk HPV $(n=38)$. Moreover, no classification into different genotypes (e.g. HPV16 and/or HPV-18) was performed. Because of the high prevalence of hrHPV infection, the study population seems unsuitable for testing hrHPV as possible prognostic factor. Moscicki et al. prospectively included 95 women with $\mathrm{CIN} 2$, of which $63 \%$ cleared their lesion after 2 years follow-up.[16] They studied the effect of HPV16/18 infection at baseline (and not all hrHPV types). They found a prognostic value of HPV16/18 with regard to disease regression in a univariable analysis, but this effect was no longer significant in a multivariable analysis. This is in accordance with our study, in which HPV 16/18 at baseline were not significantly related to disease outcome. Both results may be explained by the relatively small population, but it cannot be ruled out that $\mathrm{HPV}_{16 / 18}$ status is not a prognostic factor for disease outcome in $\mathrm{CIN}$ 2. Further research in CIN2 populations could clarify the effect of hrHPV genotypes on disease outcome. Nevertheless, given the widespread implementation of HPV-based screening for cervical cancer, the identification of additional prognostic factors should be performed in hrHPV positive lesions in order to provide with clinically relevant results.

Our study identified smoking as a prognostic factor in hrHPV positive CIN2 lesions. This is in accordance with the two previous studies that studied the influence of smoking on the natural prognosis of exclusively CIN2 lesions. Smoking is an established risk factor for the development of cervical carcinoma and has also been identified as a risk factor for the development of CIN lesions in hrHPV positive women.[18] Several biological mechanisms for this effect have been proposed, among which are an altered immune response and direct cellular effects of exposure 
to nicotine and other tobacco products. [18] The immune response in smokers is characterized by an unbalanced production of pro-and anti-inflammatory cytokines, suppression of T lymphocyte activity and lower numbers of Th cells and NK cells. Moreover, smoking women have decreased numbers of cervical Langerhans cells. An impaired immune response as a result of smoking may lead to less effective clearance of CIN lesions. Direct cellular effects of exposure to nicotine include increased cell proliferation, inhibition of apoptosis and stimulation of vascular endothelial growth factor, all of which contribute to oncogenesis and may reduce the potential of lesion regression. [18-20] Moreover, nicotine has been shown to promote cervical cell migration and invasion in vitro.[21]

These study results indicate a potential direct effect of smoking in the development of cervical dysplasia. Absence of this direct effect in non-smoking women may enable a more effective immune response, resulting in a higher likelihood of disease clearance.

Our study identified nulliparity as a prognostic factor in hrHPV positive CIN2 lesions, also after correction for age and HPV16/18 status. Previous studies with pooled data have shown a strong association between parity and cervical cancer and also an association between parity and high-grade $\mathrm{CIN}(\mathrm{CIN} 2-3)$. [22, 23] Interestingly, a recent study by Jensen et al. shows that parity is associated with an increased risk for $\mathrm{CIN}_{3}+$ in women with a persistent HPV infection. [24] This implies that the association between parity and CIN lesions may not be caused by an increased risk for HPV infection or persistence of HPV infection, but that giving birth itself seems to be a risk factor for high-grade CIN. Proposed mechanisms for this increased risk are increased hormone levels and impaired immune response, increased exposure of the transformation zone in parous women as a result of a longer ectocervical position and local tissue damage during vaginal delivery. Evidence on the association between parity and exclusively $\mathrm{CIN} 2$ lesions is scarce. Wang et al. reported on the association between parity and the risk of high-grade $\mathrm{CIN}$ development and distinguished between $\mathrm{CIN}_{2}$ and $\mathrm{CIN}_{3}$. [25] Whereas parity was found to increase the risk of $\mathrm{CIN}_{3}$, it did not increase the risk of CIN2 lesions in HPV positive women. This may imply a less effective disease clearance of $\mathrm{CIN} 2$ in parous women, leading to more cases of progressive disease. Indeed, in a study with HPV-infected women with normal cervical cytology or CIN 1, it was shown that parity is significantly and inversely associated with HPV clearance.[26] The only previous study assessing disease regression of $\mathrm{CIN} 2$ in relation to parity was performed by Miyamoto et al.[15] Nearly of women in this study were hrHPV positive. They found no differences in nulliparity between women with $\mathrm{CIN} 2$ who showed disease regression, persistence or progression. However, the mean age in the group with disease regression was higher than in the groups with persistence or progression, while correction of parity for age was not performed. This may explain why their result for parity is not in accordance with our study, which does indicate parity as a prognostic factor for regression of hrHPV positive CIN2.

A point of particular interest in studies on $\mathrm{CIN} 2$ is the diagnostic difficulty of these lesions. Studies show that $\mathrm{CIN} 2$ is a much less reproducible diagnosis than $\mathrm{CIN} 3$ and distinction between $\mathrm{CIN} 2$ and $\mathrm{CIN}_{3}$ is not always clear.[27-30] Concordance in $\mathrm{CIN} 2$ diagnosis between different pathologists was found in only $19-53 \%$ of cases, compared to $48-62 \%$ in $\mathrm{CIN} 3$ lesions. This raises discussion 
as to whether CIN 2 actually corresponds to a well-defined phase of the pathogenetic pathway of CIN, and consequently, whether it should be considered as an individual entity. Indeed, in the pathogenetic pathway of infection and transformation in $\mathrm{CIN}$, the histological image of CIN2 may represent the late stage of a productive infection or the early stage of a transforming infection, which are considered two biologically different phases in the pathogenetic pathway of infection and transformation.[31] Nevertheless, natural history studies, in which $\mathrm{CIN} 2$ and $\mathrm{CIN}_{3}$ lesions were distinguished based on conventional pathological criteria, show a much higher spontaneous regression rate for $\mathrm{CIN}_{2}(40-74 \%)$ than for $\mathrm{CIN}_{3}(22-33 \%)$. [6, 32] Other studies confirm the high regression rate of $\mathrm{CIN} 2$ lesions.[33] Based on these results, guidelines now advice upon observational management of $\mathrm{CIN} 2$ in younger women, to reduce overtreatment and associated long-term obstetric complications.[9] Despite the diagnostic difficulties, we do advocate the distinction of $\mathrm{CIN} 2$ and $\mathrm{CIN}_{3}$ lesions based on conventional histopathological criteria, with the aim to provide young women with an opportunity for observational management in case of CIN2. In this approach, any suspicion of $\mathrm{CIN} 3$ should lead to a $\mathrm{CIN}_{3}$ diagnosis, for safety concerns.

Strengths of this study include that it is the first study on prognostic factors in exclusively hrHPV positive CIN2, which makes the results applicable to current populations of women who participate in HPV-based screening for cervical cancer. Several limitations must be mentioned, however. First of all, only a limited number of women was available for inclusion in the study, as conservative management of $\mathrm{CIN} 2$ was less common in the past than currently. This may have prevented the finding of significant associations. A second limitation is the fact that our findings are based on a retrospective study of subjects at a single institution. Consequently, a selection bias cannot be ruled out. The findings of this study should therefore ideally be validated in a larger and prospective study. HPV genotyping was performed in the diagnostic biopsies, rather than in cytology specimen, as is performed in screening programs. By performing HPV genotyping in the biopsies, we ensured that the HPV genotype was actually the causative agent of the lesion.

In conclusion, we identified smoking status and parity as prognostic factors in the natural history of hrHPV positive $\mathrm{CIN} 2$, from a set of nine potential prognostic factors. These factors should be considered in individual patient counseling regarding the choice between immediate treatment or conservative management of hrHPV positive $\mathrm{CIN} 2$ lesions. Individual risk prediction could be further improved by the identification of additional prognostic factors. Further research thereto could focus on additional socio-demographic factors, such as sexual behavior, and on molecular markers of oncogenic processes. 


\section{References}

1. Munk AC, Gudlaugsson E, Ovestad IT, Lovslett K, Fiane B, Hidle B, et al. Interaction of epithelial biomarkers, local immune response and condom use in cervical intraepithelial neoplasia 2-3 regression. Gynecol Oncol. 2012;127(3):489-94.

2. Trimble CL, Piantadosi S, Gravitt P, Ronnett B, Pizer E, Elko A, et al. Spontaneous regression of high-grade cervical dysplasia: effects of human papillomavirus type and HLA phenotype. Clin Cancer Res. 2005;11(13):4717-23.

3. Holowaty P, Miller AB, Rohan T, To T. Natural history of dysplasia of the uterine cervix.] Natl Cancer Inst. 1999;91(3):252-8.

4. Discacciati MG, de Souza CA, d'Otavianno MG, Angelo-Andrade LA, Westin MC, RabeloSantos $\mathrm{SH}$, et al. Outcome of expectant management of cervical intraepithelial neoplasia grade 2 in women followed for 12 months. Eur] Obstet Cynecol Reprod Biol. 2011;155(2):2048.

5. Moore K, Cofer A, Elliot L, Lanneau G, Walker ], Gold MA. Adolescent cervical dysplasia: histologic evaluation, treatment, and outcomes. Am J Obstet Gynecol. 2007;197(2):141 e1-6.

6. Lee MH, Finlayson S], Gukova K, Hanley G, Miller D, Sadownik LA. Outcomes of Conservative Management of High Grade Squamous Intraepithelial Lesions in Young Women.] Low Genit Tract Dis. 2018;22(3):212-8.

7. Kyrgiou M, Athanasiou A, Kalliala IE], Paraskevaidi M, Mitra A, Martin-Hirsch PP, et al. Obstetric outcomes after conservative treatment for cervical intraepithelial lesions and early invasive disease. Cochrane Database Syst Rev. 2017;11:CD012847.

8. Group T, Sharp L, Cotton S, Cochran C, Gray N, Little ], et al. After-effects reported by women following colposcopy, cervical biopsies and LLETZ: results from the TOMBOLA trial. BJOC. 2009;116(11):1506-14.

9. Massad LS, Einstein MH, Huh WK, Katki HA, Kinney WK, Schiffman M, et al. 2012 updated consensus guidelines for the management of abnormal cervical cancer screening tests and cancer precursors. J Low Genit Tract Dis. 2013;17(5 Suppl 1):S1-S27.

10. Ho GY, Einstein MH, Romney SL, Kadish AS, Abadi M, Mikhail M, et al. Risk factors for persistent cervical intraepithelial neoplasia grades 1 and 2: managed by watchful waiting. ] Low Genit Tract Dis. 2011;15(4):268-75.

11. Munk AC, Gudlaugsson E, Malpica A, Fiane B, Lovslett KI, Kruse A], et al. Consistent condom use increases the regression rate of cervical intraepithelial neoplasia 2-3. PLoS One. 2012;7(9):e45114.

12. Sole-Sedeno JM, Mancebo G, Miralpeix E, Lloveras B, Bellosillo B, Alameda F, et al. Utility of Human Papillomavirus Cenotyping in the Management of Low-Crade Squamous Intraepithelial Lesions. J Low Genit Tract Dis. 2018;22(1):13-6.

13. Koeneman MM, Kruitwagen RF, Nijman HW, Slangen BF, Van Corp T, Kruse A]. Natural history of high-grade cervical intraepithelial neoplasia: a review of prognostic biomarkers. Expert Rev Mol Diagn. 2015;15(4):527-46.

14. Loopik DL, Doucette S, Bekkers RL, Bentley JR. Regression and Progression Predictors of CIN2 in Women Younger Than 25 Years. J Low Cenit Tract Dis. 2016;20(3):213-7. 
15. Miyamoto S, Hasegawa ], Morioka M, Hirota Y, Kushima M, Sekizawa A. The association between p16 and Ki67 immunohistostaining and the progression of cervical intraepithelial neoplasia grade 2. Int ] Gynaecol Obstet. 2016;134(1):45-8.

16. Moscicki AB, Ma Y, Wibbelsman C, Darragh TM, Powers A, Farhat S, et al. Rate of and risks for regression of cervical intraepithelial neoplasia 2 in adolescents and young women. Obstet Gynecol. 2010;116(6):1373-80.

17. Koeneman MM, van Lint FHM, van Kuijk SM], Smits LJM, Kooreman LFS, Kruitwagen R, et al. A prediction model for spontaneous regression of cervical intraepithelial neoplasia grade 2 , based on simple clinical parameters. Hum Pathol. 2017;59:62-9.

18. Fonseca-Moutinho JA. Smoking and cervical cancer. ISRN Obstet Cynecol. 2011;2011:847684.

19. Guillaud M, Buys TP, Carraro A, Korbelik ], Follen M, Scheurer M, et al. Evaluation of HPV infection and smoking status impacts on cell proliferation in epithelial layers of cervical neoplasia. PLoS One. 2014;9(9):e107088.

20. Samir R, Asplund A, Tot T, Pekar C, Hellberg D. Tissue tumor marker expression in smokers, including serum cotinine concentrations, in women with cervical intraepithelial neoplasia or normal squamous cervical epithelium. Am ] Obstet Gynecol. 2010;202(6):579 e1-7.

21. Wang C, Gu W, Zhang Y, Ji Y, Wen Y, Xu X. Nicotine promotes cervical carcinoma cell line HeLa migration and invasion by activating Pl3k/Akt/NF-kappaB pathway in vitro. Exp Toxicol Pathol. 2017;69(6):402-7.

22. Munoz N, Franceschi S, Bosetti C, Moreno V, Herrero R, Smith JS, et al. Role of parity and human papillomavirus in cervical cancer: the IARC multicentric case-control study. Lancet. 2002;359(9312):1093-101.

23. International Collaboration of Epidemiological Studies of Cervical C. Cervical carcinoma and reproductive factors: collaborative reanalysis of individual data on 16,563 women with cervical carcinoma and 33,542 women without cervical carcinoma from 25 epidemiological studies. Int ] Cancer. 2006;119(5):1108-24.

24. Jensen KE, Schmiedel S, Norrild B, Frederiksen K, Iftner T, Kjaer SK. Parity as a cofactor for high-grade cervical disease among women with persistent human papillomavirus infection: a 13-year follow-up. Br] Cancer. 2013;108(1):234-9.

25. Wang SS, Zuna RE, Wentzensen N, Dunn ST, Sherman ME, Gold MA, et al. Human papillomavirus cofactors by disease progression and human papillomavirus types in the study to understand cervical cancer early endpoints and determinants. Cancer Epidemiol Biomarkers Prev. 2009;18(1):113-20.

26. Kim JW, Song SH, Jin CH, Lee JK, Lee NW, Lee KW. Factors affecting the clearance of high-risk human papillomavirus infection and the progression of cervical intraepithelial neoplasia. ] Int Med Res. 2012;40(2):486-96.

27. Ismail SM, Colclough AB, Dinnen JS, Eakins D, Evans DM, Gradwell E, et al. Observer variation in histopathological diagnosis and grading of cervical intraepithelial neoplasia. BM]. 1989;298(6675):707-10.

28. Carreon JD, Sherman ME, Guillen D, Solomon D, Herrero R, Jeronimo ], et al. CIN2 is a much less reproducible and less valid diagnosis than $\mathrm{CIN} 3$ : results from a histological review of population-based cervical samples. Int ] Gynecol Pathol. 2007;26(4):441-6. 
29. Dalla Palma P, Giorgi Rossi P, Collina G, Buccoliero AM, Ghiringhello B, Gilioli E, et al. The reproducibility of CIN diagnoses among different pathologists: data from histology reviews from a multicenter randomized study. Am ] Clin Pathol. 2009;132(1):125-32.

30. Cai B, Ronnett BM, Stoler M, Ferenczy A, Kurman R], Sadow D, et al. Longitudinal evaluation of interobserver and intraobserver agreement of cervical intraepithelial neoplasia diagnosis among an experienced panel of gynecologic pathologists. Am J Surg Pathol. 2007;31(12):185460.

31. Steenbergen RD, Snijders P], Heideman DA, Meijer C]. Clinical implications of (epi)genetic changes in HPV-induced cervical precancerous lesions. Nat Rev Cancer. 2014;14(6):395-405.

32. Ostor AC. Natural history of cervical intraepithelial neoplasia: a critical review. Int ] Cynecol Pathol. 1993;12(2):186-92.

33. Tainio K, Athanasiou A, Tikkinen KAO, Aaltonen R, Cardenas ], Hernandes, et al. Clinical course of untreated cervical intraepithelial neoplasia grade 2 under active surveillance: systematic review and meta-analysis. BM]. 2018;360:k499. 


\section{。}

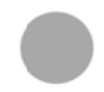

\section{PART 2}

Assessment of clinical applicability of imiquimod cream as a treatment modality for high-grade CIN 


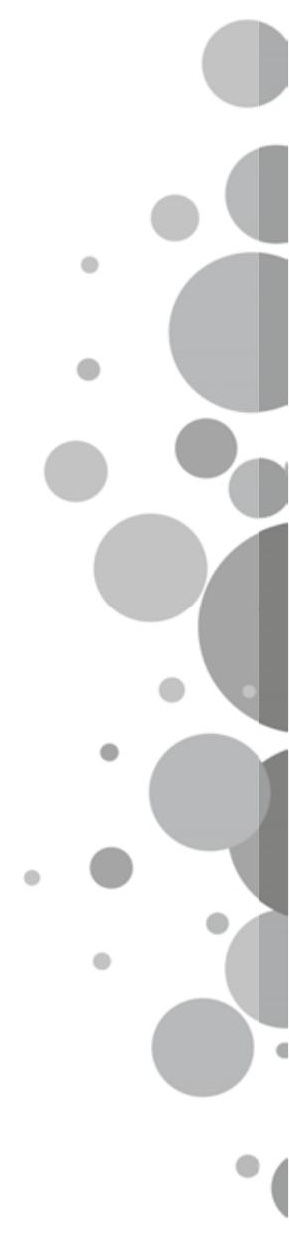




\section{CHAPTER 7}

Physicians' awareness, attitudes and experiences rogarding imiquimod treatment of vaginal and cervical intraepithelial neoplasia

Margot M Koeneman, Anna JM van de Sande, Heleen ] van Beekhuizen, Kees C Cerestein, Rafli van de Laar, Roy FPM Kruitwagen, Arnold-Jan Kruse Journal of Lower Cenital Tract Disease. 2016: Jan;20(1):75-9 


\section{Abstract}

Objective: The aim of the study was to assess awareness, attitudes and current clinical experiences of gynecologists regarding imiquimod as a potential treatment modality for vaginal intraepithelial neoplasia (VAIN) and cervical intraepithelial neoplasia (CIN).

Methods: A 37-item questionnaire consisting of both multiple choice and open questions was sent to all Dutch gynecologists who regularly perform colposcopies in all 87 Dutch hospitals, in December 2014. The outcomes were assessed using descriptive statistics.

Results: Gynecologists from 52 hospitals (60\%) completed the questionnaire. Of the 77 respondents, $79 \%$ and $58 \%$ were aware of imiquimod for treating VAIN and CIN, respectively. Twelve and five respondents had used imiquimod to treat VAIN and CIN, respectively; most treatments were for intractable VAIN lesions, recurrent lesions and to avoid surgical treatment for $\mathrm{CIN}$ in patients with a future pregnancy wish. Most respondents reported successful treatment outcomes but frequent side effects. Most (96\%) stated that they would consider using imiquimod to treat high-grade $\mathrm{CIN}$ in selected patients, but only upon additional evidence and inclusion into treatment guidelines.

Conclusions: The awareness of imiquimod as a potential treatment for VAIN and CIN was limited, probably because of the paucity of evidence regarding vaginal imiquimod efficacy, the lack of inclusion into guidelines, and the high frequency of side effects. Imiquimod was applied off-label in a limited number of selected patients, with good treatment results. The respondents generally had a positive attitude towards treating VAIN and CIN with imiquimod. Additional evidence on treatment efficacy and inclusion in treatment guidelines is necessary before application in clinical practice. 


\section{Introduction}

Imiquimod is a toll-like receptor antagonist that has antiviral and antitumor properties. Imiquimod cream is currently registered for the topical treatment of certain types of basal cell carcinoma, actinic keratosis, and genital warts. It is also an effective treatment modality for HPVinduced vulvar intraepithelial neoplasia (VIN).[1] Indeed, imiquimod therapy is being applied in VIN in order to reduce potentially mutilating effects of surgical therapy and is recommended in current treatment guidelines. [2, 3]

Imiquimod has also been studied as a treatment modality for HPV-induced vaginal intraepithelial neoplasia (VAIN) and cervical intraepithelial neoplasia (CIN), in order to reduce the side effects and challenges associated with the standard treatment modalities for these conditions. At present, advised treatment modalities for high-grade CIN include ablation or excision of the transformation zone or hysterectomy in selected patients with recurrent or persistent highgrade CIN. [4] When treatment is indicated, this is usually performed by large loop excision of the transformation zone (LLETZ). LLETZ associates with moderately severe short-term side effects, including prolonged bleeding and vaginal discharge. An important long-term side effect of LLETZ treatment is a twofold increase in the risk of premature birth in subsequent pregnancies. [5, 6] For this reason, management by observation is recommended for young women with either $\mathrm{CIN} 2$ or $\mathrm{CIN}_{3}$ and adequate colposcopy.[4] The preferred treatment modalities for VAIN are surgical excision and laser ablation. However, VAIN treatment can be challenging as the lesions can be large and scattered. As a result, the rates of residual and recurrent VAIN after primary treatment are high.[7, 8]

Treatment efficacy of imiquimod in VAIN and CIN has been examined in several studies.[9-13] Although the patient populations and outcome measures in these studies were heterogeneous, the results were generally promising. One of these studies was a randomized controlled trial (RCT): it showed that in 22 out of $30(73 \%)$ patients with high-grade $\mathrm{CIN}$, imiquimod treatment caused histological regression to $\mathrm{CIN} 1$ or less. However, side effects of vaginal application of imiquimod were common.[11]

Imiquimod is not currently registered for the treatment of VAIN and CIN and is not part of treatment guidelines. Additional studies on treatment efficacy and clinical applicability are currently conducted, but awareness and attitudes of physicians regarding imiquimod treatment for VAIN and CIN are unknown. In both research and clinical implementation of a new treatment modality, awareness and attitudes of physicians towards the treatment modality may be important factors for success. Additionally, knowledge on current off-label clinical experiences may provide valuable additional information on the clinical applicability of imiquimod in VAIN and $\mathrm{CIN}$ and perceived treatment indications.

This study aims to assess awareness, attitudes and current clinical experience of gynecologists with regard to imiquimod treatment of VAIN and CIN. 


\section{Materials and methods}

The study design and questionnaire were developed according to Checklist for Reporting Results of Internet E-Surveys (CHERRIES) guidelines.[14] A completed CHERRIES guideline has been attached as a supplement (Supplemental Digital Content 1). Since this study is a survey among physicians, ethical approval was not necessary.

\section{Population}

The electronic survey was sent to all gynecologists in the Netherlands who regularly perform colposcopies and treat VAIN and CIN lesions. Since a specific mailing list was not available, all hospitals in the Netherlands in which colposcopies are conducted $(n=87)$ were contacted by telephone to identify these gynecologists. Of the 87 hospitals, 8 were university hospitals, 37 were large semi-specialized teaching hospitals, and 42 were general non-teaching hospitals. In total, 176 gynecologists were identified and contacted by an e-mail containing an invitation message and a link to the survey. The first invitation was sent in November 2014. Reminders were sent after 3 and 6 weeks to only those gynecologists who had not responded (as tracked by the survey program). The survey was closed after 9 weeks.

\section{Questionnaire}

An anonymous electronic questionnaire was designed by the authors and was conducted by using a commercial web survey provider (www.surveymonkey.com). The questionnaire has been attached as a supplement (Supplemental Digital Content 2). The questionnaire was composed by an expert panel of four gynecological oncologists with experience in colposcopy and treatment of VAIN and CIN. The questionnaire was tested by five colleagues; this led to several changes to the questionnaire. The questionnaire consisted of 37 items relating to current colposcopy practice, awareness of imiquimod as an immunotherapy for genital warts and VIN lesions, and as an experimental treatment modality of VAIN and CIN lesions, whether the gynecologist had used imiquimod to treat VAIN and CIN and what the outcomes were (only when relevant), and opinions about imiquimod use for high-grade CIN lesions. The questions concerning current colposcopy practice were included to determine whether the physicians were aware of the risk of premature birth after LLETZ and their clinical practice with regard to this potential adverse effect. The majority of questions were multiple-choice, and it was possible to make a comment. The survey system automatically stored all responses in a database. E-mail addresses of all respondents were stored by the survey system for the purpose of sending reminder messages to non-responders. Respondents could only complete the questionnaire once.

\section{Statistics}

The descriptive statistics were generated by using SPSS (IBM Corp., released 2012, IBM SPSS Statistics for Windows, Version 21.0, Armonk, NY). 


\section{Results}

Gynecologists from 52 hospitals (60\%) responded to the questionnaire. The questionnaire was started by 79 participants and was completed by 77 participants (44\%). The two incomplete questionnaires were completely blank and were therefore excluded from the analysis. The professional function, type of hospital, and number of colposcopies that each gynecologist conducted or supervised yearly are displayed in Table 1. All respondents were aware that imiquimod could be used to treat genital warts and thus were familiar with the drug.

Table 1. Professional function, type of hospital, and number of colposcopies performed by the respondents

\begin{tabular}{|c|c|c|c|}
\hline 1. & What is your professional function? & $\mathbf{n}$ & $\%$ \\
\hline- & Gynecologic oncologist or fellow gynecologic oncologist & 13 & $(17 \%)$ \\
\hline- & Gynecologist with semi-specialization in gynecologic oncology & 41 & $(53 \%)$ \\
\hline- & Gynecologist with other (semi-) specialization & 23 & $(30 \%)$ \\
\hline 2. & In what hospital type are you currently employed? & & \\
\hline- & University hospital or specialized oncological center & 12 & $(16 \%)$ \\
\hline- & Semi-specialized teaching hospitals & 42 & $(55 \%)$ \\
\hline- & Non-teaching hospital & 23 & $(30 \%)$ \\
\hline 3. & How many colposcopies do you conduct or supervise yearly? & & \\
\hline- & Less than 30 & 6 & $(8 \%)$ \\
\hline- & $30-100$ & 40 & (52\%) \\
\hline- & More than 100 & 31 & $(40 \%)$ \\
\hline
\end{tabular}

\section{Current colposcopy practice}

A large subset of respondents tailor their colposcopy practice to the individual patient: 32 respondents $(42 \%)$ perform diagnostic biopsies in patients who wish to become pregnant and apply a see-and-treat policy in patients without a future pregnancy wish. Thirty respondents (39\%) never apply a see-and treat policy, while ten respondents (13\%) always apply a see-andtreat policy. The majority of respondents $(n=52,68 \%)$ considered the risk of premature birth after LLETZ to be unchanged or only marginally increased. The other respondents estimated the relative risk at approximately 2.7 (range, 2-6). Of all the respondents, $53(69 \%)$ always discuss this risk with patients and 15 (19\%) never discuss this risk.

\section{Awareness of imiquimod as immunotherapy for VIN, VAIN and CIN}

The vast majority of respondents $(n=70,91 \%)$ were aware that imiquimod could be used to treat VIN. Sixty-one (79\%) respondents were aware of the application of imiquimod for VAIN, in literature or clinical practice. Forty-five (58\%) respondents were aware of the application of imiquimod for $\mathrm{CIN}$, in literature or clinical practice. Respondents who performed more than 100 colposcopies yearly and (fellow) gynecologic oncologists or semi-specialized gynecologic oncologists associated with a higher level of awareness in all cases.

Results are displayed in table 2 . 
Table 2: Awareness and off-label use of imiquimod as an immunotherapy for VIN, VAIN, and $\mathrm{CIN}$ by respondents divided according to hospital type

\begin{tabular}{lllll}
\hline & $\begin{array}{l}\text { Total respondent } \\
\text { population } \\
(\mathbf{n = 7 7 )}\end{array}$ & $\begin{array}{l}\text { University } \\
\text { hospital } \\
(\mathbf{n = 1 2})\end{array}$ & $\begin{array}{l}\text { Semi-specialized } \\
\text { teaching hospital } \\
(\mathbf{n = 4 2 )}\end{array}$ & $\begin{array}{l}\text { Non-teaching } \\
\text { hospital } \\
(\mathbf{n = 2 3})\end{array}$ \\
\hline Aware of imiquimod in VIN & $70(91 \%)$ & $12(100 \%)$ & $39(93 \%)$ & $19(83 \%)$ \\
Aware of imiquimod in VAIN & $61(79 \%)$ & $12(100 \%)$ & $36(81 \%)$ & $15(65 \%)$ \\
Aware of imiquimod in CIN & $45(58 \%)$ & $11(92 \%)$ & $28(67 \%)$ & $6(26 \%)$ \\
Applied imiquimod in VAIN & $12(16 \%)$ & $4(33 \%)$ & $6(14 \%)$ & $3(9 \%)$ \\
Applied imiquimod in CIN & $5(7 \%)$ & $2(17 \%)$ & $2(5 \%)$ & $1(4 \%)$ \\
\hline
\end{tabular}

$\mathrm{CIN}$, cervical intraepithelial neoplasia; VAIN, vaginal intraepithelial neoplasia; VIN, vulvar intraepithelial neoplasia.

\section{Off-label application of imiquimod in VAIN and CIN}

Twelve (16\%) and five (7\%) respondents had used imiquimod to treat VAIN and CIN lesions, respectively (table 2). Clinical application of imiquimod was more frequent in respondents who performed more than 100 colposcopies yearly and (fellow) gynecologic oncologists or semispecialized gynecologic oncologists, compared to the total respondent population. Frequency of application and lesion characteristics are displayed in table 3. Most respondents applied it less than 5 times in VAIN and less than 10 times in CIN. Imiquimod was applied in both low grade and high-grade lesions and in both primary and recurrent lesions.

Table 3: Off-label application of imiquimod in VAIN and CIN by the respondents: frequency, lesion characteristics, and treatment success

\begin{tabular}{|c|c|c|c|c|c|}
\hline & & \multicolumn{2}{|c|}{ VAIN $(n=12)$} & \multicolumn{2}{|c|}{$\operatorname{CIN}(n=5)$} \\
\hline \multicolumn{6}{|c|}{ How often have you applied imiquimod for this indication? } \\
\hline - & $1-5$ times & 10 & $(83 \%)$ & 3 & $(60 \%)$ \\
\hline - & 6-10 times & 1 & $(8 \%)$ & 2 & $(40 \%)$ \\
\hline - & More than 10 times & 1 & $(8 \%)$ & 0 & - \\
\hline \multicolumn{6}{|c|}{ What was (were) the lesion grade(s)? } \\
\hline- & Low-grade lesion(s) (VAIN/CIN 1) & 2 & $(16 \%)$ & 1 & $(20 \%)$ \\
\hline- & High-grade lesion(s) (VAIN/CIN 2-3) & 7 & $(58 \%)$ & 3 & $(60 \%)$ \\
\hline- & Both & 3 & $(25 \%)$ & 1 & $(20 \%)$ \\
\hline \multicolumn{6}{|c|}{ Was it a primary or recurrent lesion? } \\
\hline- & Primary lesion(s) & 4 & $(33 \%)$ & 1 & $(20 \%)$ \\
\hline- & Recurrent lesion(s) & 4 & $(33 \%)$ & 3 & $(60 \%)$ \\
\hline- & Both & 4 & $(33 \%)$ & 1 & $(20 \%)$ \\
\hline \multicolumn{6}{|c|}{$\begin{array}{l}\text { Was treatment of the lesion(s) with imiquimod successful? } \\
\text { (multiple options possible)* }\end{array}$} \\
\hline - & $\begin{array}{l}\text { Yes, lesion regression or complete remission, invasive } \\
\text { therapy no longer needed }\end{array}$ & 9 & $(75 \%)$ & 5 & $(100 \%)$ \\
\hline - & No, persistent lesion & 3 & $(25 \%)$ & 1 & $(20 \%)$ \\
\hline - & No, progressive lesion & 1 & $(8 \%)$ & 0 & \\
\hline
\end{tabular}




\section{$\operatorname{VAIN~}(n=12) \quad \operatorname{CIN}(n=5)$}

Would you recommend treatment of VAIN/CIN with imiquimod, and would you apply it again?

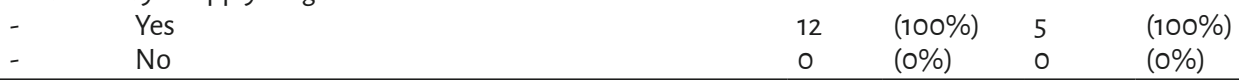

* Percentages add up to $>100 \%$ because some respondents treated more than one patient and reported on both disease regression and persistence or progression.

$\mathrm{CIN}$, cervical intraepithelial neoplasia; VAIN, vaginal intraepithelial neoplasia.

The most common reasons for choosing imiquimod to treat VAIN were recurrence after laser and/or excisional therapy $(n=5)$ and the estimation that laser or excisional therapy would be too difficult or impossible $(n=4)$. The reasons for choosing imiquimod to treat $\mathrm{CIN}$ were recurrence after LLETZ $(n=3)$, large CIN lesions that demanded a large LLETZ in patients with a future pregnancy wish $(n=1)$, and refusal by the patient to undergo LLETZ $(n=1)$.

In both VAIN and CIN, imiquimod was applied via vaginal capsules, vaginal suppositories, vaginal tampons, or vaginal applicators. The respondents used a fairly consistent treatment dose and protocol: it generally consisted of 2-3 applications per week for 12-16 weeks. The exception was one respondent who applied imiquimod five times per week for 6 weeks to treat VAIN. All respondents used one $6.25 \mathrm{mg}$ sachet per application.

Treatment efficacy was mostly evaluated by colposcopy, with diagnostic biopsies on indication. Four respondents combined colposcopy with cervical cytology after imiquimod treatment of VAIN. One respondent performed cervical cytology only after imiquimod treatment of CIN.

The treatment outcome was documented by 11 of the 12 respondents for VAIN and by all five respondents for $\mathrm{CIN}$ (table 3). Nine respondents (75\%) experienced treatment success in VAIN and all five respondents experienced treatment success in $\mathrm{CIN}$ : invasive therapy was no longer needed. Only one respondent reported disease progression, in VAIN. All respondents would apply the therapy again. Since this study did not aim to systematically document treatment outcome, overall treatment efficacy cannot be derived from the results. Side effects of imiquimod treatment of VAIN and CIN were common: all but one of the respondents reported that the treatment had side effects. The side effects consisted of vaginal and vulvar pain, vulvar erythema, vulvar erosion and/or ulceration, vaginal discharge, flu-like symptoms, arthralgia, and fatigue. The side effects were subjectively graded by the respondents and were generally considered to be mild to moderate; only one respondent documented severe side effects. Nevertheless, five respondents stated that at least one of their patients had stopped treatment due to the side effects (three for VAIN patients and two for CIN patients).

Taking into consideration the efficacy and side effects of imiquimod treatment, all respondents stated that they would use imiquimod again in patients with VAIN and CIN.

\section{Future application of imiquimod}

All participants were informed in the questionnaire of the results of a recent RCT on the efficacy of imiquimod in high-grade $\mathrm{CIN}$.[11] On the condition that the study results were validated in 
a larger patient population, 74 respondents (96\%) stated that they would consider applying imiquimod in high-grade CIN. Thirteen respondents also commented on this question. Several respondents stressed that it is important that additional high-quality evidence of treatment efficacy becomes available; they also emphasized the need for additional research on the longterm effects, recurrence rates, and cost-effectiveness of imiquimod therapy for VAIN and CIN. Two respondents stated that imiquimod treatment would have to be incorporated in national guidelines on treatment of VAIN and CIN before they would apply it. One respondent commented that he/she would consider applying imiquimod in CIN 2 but not in CIN 3. Several respondents were concerned about the clinical applicability of imiquimod in $\mathrm{CIN}$ : they questioned whether the treatment would be tolerable for patients due to the common side effects, relatively long treatment duration, and practicalities such as the mode of application. One respondent stated that patient perspectives regarding imiquimod treatment for gynecological conditions should be researched further. Most respondents $(n=72,94 \%)$ stated that they would consider participating in a RCT on the treatment efficacy of imiquimod in high-grade CIN.

\section{Discussion}

The current study shows that there is limited awareness among gynecologists in the Netherlands regarding investigational treatment of VAIN and CIN with imiquimod; this was especially true for CIN. A small number of respondents had used imiquimod as an off-label treatment for VAIN and CIN. The main reasons for their off-label application of imiquimod were recurrence of VAIN and CIN lesions, VAIN lesions that could not otherwise be optimally treated, and to avoid surgical treatment in patients with CIN who had a future pregnancy wish. The respondents reported good treatment efficacy but also stated that the side effects of imiquimod therapy were common. Nevertheless, all gynecologists who had treated patients with imiquimod stated that they would use this treatment modality again in the future. The attitude of gynecologists regarding imiquimod as a potential treatment of high-grade CIN is generally positive: a vast majority of participating gynecologists are willing to apply imiquimod for high-grade $\mathrm{CIN}$, but recommend additional trials and subsequent inclusion of this therapy in $\mathrm{CIN}$ guidelines.

The limited awareness and clinical application of imiquimod in VAIN and CIN may be explained by the relative paucity of evidence regarding the efficacy of imiquimod treatment in VAIN and CIN and the fact that imiquimod treatment is not currently part of treatment guidelines. There are only three studies on the efficacy of imiquimod treatment in VAIN, and two of these focused on only or mainly low-grade lesions. $[9,13]$ The remaining study focused only on patients with high-grade lesions, but the patient population was small: disease regression was observed in six of the seven patients. [10] There are also only three studies on the efficacy of imiquimod in CIN. One of these was a recent RCT that showed 22 out of 30 (73\%) patients with high-grade CIN exhibited disease regression after treatment. [11] The two other studies focused on different outcome measures or had a heterogeneous patient population. $[12,13]$ Large-scale RCTs that confirm treatment efficacy and assess disease recurrence over the long term are lacking. Additional evidence may lead to inclusion of imiquimod in treatment guidelines, leading to increased awareness and clinical application. 
Indeed, several respondents stressed the need for additional evidence regarding treatment efficacy, side effects, and long-term outcomes, and stated that they would only use imiquimod to treat VAIN and CIN when it was incorporated in the treatment guidelines.

An important limitation to the clinical application of imiquimod may be the high rate of side effects of vaginal imiquimod therapy. All trials on vaginal imiquimod treatment and the current study show high rates of side effects. These consist mainly of vaginal discharge, flu-like symptoms and vulvar pain or pruritus, which were reported in up to $32 \%, 93 \%$ and $93 \%$ respectively in previous trials on vaginal imiquimod for CIN lesions.[11, 12] Vulvar erosion or ulceration was seen in up to $33 \%$ of patients treated with vaginal imiquimod. Flu-like symptoms and vulvar pain or pruritus are not common side effects of LLETZ treatment, which is more common associated with prolonged vaginal bleeding and vaginal discharge. An observational study in 185 patients who underwent LLETZ treatment showed that $40 \%$ of women experienced moderate to severe discharge and 50\% experienced moderate to severe bleeding. Bleeding persisted for more than two weeks in approximately $40 \%$ of women.[15] Intervention for postoperative hemorrhage is rare: 7 cases (1.3\%) were reported in a retrospective cohort study among 557 patients. [16] Indeed, several respondents in the current study who had not used vaginal imiquimod expressed their concern about its potential side effects. When choosing imiquimod therapy for VAIN or CIN, the side effects of standard treatment modalities should outweigh the side effects of imiquimod therapy, making imiquimod an acceptable treatment alternative. As such, imiquimod treatment may have to be limited to those patients who cannot be optimally treated by standard treatment modalities or in whom surgery is to be avoided. The latter group will consist mainly of younger patients with high-grade $\mathrm{CIN}$ and a future pregnancy wish. In order to reduce overtreatment, observation according to the ASCCP guideline should be considered first. [4] Imiquimod could be an alternative if treatment is preferred or upon disease persistence. This conclusion was clearly shared by the respondents in the current study who had used imiquimod to treat VAIN or CIN: they only used this treatment for VAIN lesions that could not be optimally treated by laser ablation or surgery, recurrent VAIN or CIN lesions, and patients with CIN lesions who had a future pregnancy wish and who would be at risk of premature birth if they underwent LLETZ treatment. Interestingly, the majority of our respondents underestimated the risk of premature birth in subsequent pregnancies. Moreover, a significant number stated that they do not discuss this risk with patients before LLETZ treatment. More widespread awareness of this risk could increase the clinical application of imiquimod after confirmation of its treatment modality and inclusion in treatment guidelines.

Treatment experiences in Dutch clinical practice were generally good. Off-label clinical application of imiquimod in VAIN and CIN was performed by a limited number of respondents, of whom the majority experienced successful treatment outcomes. Interestingly, our study also showed that there was a high rate of treatment discontinuation due to side effects. The incidence seems higher than that reported in previous trials, in which treatment discontinuation was very low. This discrepancy may reflect a better dosing regimen and response to side effects (with medication and dosing changes) in the clinical trials that arose from increased experience with this therapy. Quality of life studies or patient preference studies concerning vaginal imiquimod therapy are 
currently not available, but seem necessary to determine the clinical applicability of imiquimod therapy. Nevertheless, despite the high rate of side effects, the respondents of the current study generally considered imiquimod treatment of VAIN and CIN to be successful.

The current study is the first to provide insights regarding the awareness, off-label application, and attitudes of gynecologists with regard to imiquimod treatment of VAIN and CIN. The study has several limitations. First, although the questionnaire was tested before application, formal validation has not been performed. A pilot test was considered to be infeasible considering the small target population. However, we do not believe that the lack of validation influenced the response rate, because only two respondents did not finish the questionnaire, and those that did not respond at all could not have seen the questionnaire. Second, participation in the survey was voluntary. As a result, respondents who have a special interest or opinion with regard to the subject may have been more likely to participate, thus resulting in selection bias. The last limitation may be the moderate response rate of the study However, this response rate is comparable to the response rates of other surveys on the knowledge and attitudes of physicians with regard to several medical issues. $[17,18,19]$ Moreover, the absolute number of respondents is substantial. In addition, the survey encompassed the majority of the hospitals that were contacted (60\%).Thus, the results of this survey seem to be generally reliable and valuable.

\section{Conclusions}

The responding gynecologists had limited awareness of imiquimod as an experimental treatment modality for VAIN and CIN. Far fewer actually used this treatment in clinical practice. This is probably due to the limited evidence of imiquimod efficacy for these diseases, the fact that imiquimod treatment has not yet been incorporated in treatment guidelines, and the common side effects of the therapy. As a result, off-label application of imiquimod is currently limited to selected patients, if the benefits of imiquimod therapy outweighed its side effects. The treatment results in these patients were generally good. To facilitate more widespread implementation of imiquimod in clinical practice, more evidence regarding its efficacy, clinical applicability, and long-term outcomes is needed, followed by incorporation of imiquimod as a treatment modality in treatment guidelines. Treatment efficacy, clinical applicability, and long-term outcomes will be addressed by the authors in a randomized controlled trial: TOPIC trial (TOPical treatment with Imiquimod of high-grade CIN, ClinicalTrial.gov identifier: NCT02329171).[20] This trial aims to confirm and assess the efficacy of vaginal imiquimod treatment for $\mathrm{CIN}$ and to provide additional evidence on the rate of disease recurrence and the quality of life of the patients during and after this treatment. 


\section{References}

1. van Seters $M$, van Beurden $M$, ten Kate F], et al. Treatment of vulvar intraepithelial neoplasia with topical imiquimod. T N Engl ] Med. 2008;358:1465-1473.

2. Management of vulvar intraepithelial neoplasia. Committee Opinion No. 509. American College of Obstetricians and Gynecologists. Obstet Cynecol 2011;118:1192-1194.

3. Royal College of Obstetricians and Gynaecologists. Green-top Guideline No. 58: The Management of Vulval Skin Disorders. London: RCOG; 2011. [cited 2015 Aug 10] Available at: https://www.rcog.org.uk/globalassets/documents/guidelines/gtg_58.pdf.

4. Massad LS, Einstein MH, Huh WK, et al. 2012 updated consensus guidelines for the management of abnormal cervical cancer screening tests and cancer precursors. ] Low Genit Tract Dis. 2013;17(5 Suppl 1):S1-S27.

5. Crane JM. Pregnancy outcome after loop electrosurgical excision procedure: a systematic review. Obstet Gynecol. 2003;102:1058-1062.

6. Kyrgiou M, Koliopoulos G, Martin-Hirsch P, et al. Obstetric outcomes after conservative treatment for intraepithelial or early invasive cervical lesions: systematic review and metaanalysis. Lancet. 2006;367:489-498

7. Gurumurthy M, Cruickshank ME. Management of vaginal intraepithelial neoplasia. ] Low Genit Tract Dis. 2012;16:306-312.

8. Gunderson CC, Nugent EK, Elfrink SH, Cold MA, Moore KN. A contemporary analysis of epidemiology and management of vaginal intraepithelial neoplasia. Am J Obstet Cynecol. 2013;208:410.e1-6.

9. Buck HW, Guth KJ. Treatment of vaginal intraepithelial neoplasia (primarily low grade) with imiquimod 5\% cream. J Low Cenit Tract Dis. 2003;3:290-293.

10. Haidopoulos D, Diakomanolis E, Rodolakis A, et al. Can local application of imiquimod cream be an alternative mode of therapy for patients with high-grade intraepithelial lesions of the vagina? Int ] Gynecol Cancer. 2005;15:898-902.

11. Grimm C, Polterauer S, Natter C, et al. Treatment of cervical intraepithelial neoplasia with topical imiquimod: a randomized controlled trial. Obstet Gynecol. 2012;120:152-159.

12. Pachman DR, Barton DL, Clayton AC, et al. Randomized clinical trial of imiquimod: an adjunct to treating cervical dysplasia. Am J Obstet Gynecol. 2012;206:42 e1-7.

13. Lin CT, Qiu JT, Wang C], et al. Topical imiquimod treatment for human papillomavirus infection in patients with and without cervical/vaginal intraepithelial neoplasia. Taiwan ] Obstet Gynecol. 2012;51:533-538.

14. Eysenbach C. Improving the quality of Web surveys: the Checklist for Reporting Results of Internet E-Surveys (CHERRIES). ] Med Internet Res. 2004;6:e34.

15. Group T, Sharp L, Cotton S, et al. After-effects reported by women following colposcopy, cervical biopsies and LLETZ: results from the TOMBOLA trial. B]OG. 2009;116:1506-14.

16. Dunn TS, Killoran K, Wolf D. Complications of outpatient LLETZ procedures. J Reprod Med. 2004;49:76-8.

17. England L], Anderson BL, Tong VT, et al. Screening practices and attitudes of obstetriciansgynecologists toward new and emerging tobacco products. Am ] Obstet Cynecol. 2014;211:695. e1-7. 
18. Fox E, McCuaig ], Demsky R, et al. The sooner the better: Cenetic testing following ovarian cancer diagnosis. Gynecol Oncol. 2015;137:423-429.

19. Kissin DM, Power ML, Kahn EB, et al. Attitudes and practices of obstetrician-gynecologists regarding influenza vaccination in pregnancy. Obstet Gynecol. 2011;118:1074-1080. 20. Maastricht University Medical Center. TOPical Imiquimod Treatment of High-grade Cervical Intraepithelial Neoplasm: a Randomized Controlled Trial. In: ClinicalTrials. gov [Internet]. Bethesda (MD): National Library of Medicine (US). 2000- [cited 2015 Aug 10]. Available from: https://clinicaltrials.gov/ct2/show/NCT02329171; NLM Identifier: NCT02329171. 
Supplemental digital content 1.

Checklist for Reporting Results of Internet E-Surveys (CHERRIES)

\begin{tabular}{|c|c|c|}
\hline Design & Survey design & $\begin{array}{l}\text { The survey was intended for all gynaecologists in the } \\
\text { Netherlands who regularly perform colposcopies and } \\
\text { treat CIN/VAIN. Because a specific mailing list was not } \\
\text { available, all hospitals in the Netherlands were contacted } \\
\text { by phone to identify these specialists. }\end{array}$ \\
\hline \multirow{3}{*}{$\begin{array}{l}\text { IRB (Institutional Review } \\
\text { Board) approval and } \\
\text { informed consent process }\end{array}$} & IRB approval & IRB approval was not necessary for this study. \\
\hline & Informed consent & $\begin{array}{l}\text { The participants were contacted by email. The } \\
\text { introduction email contained information about the } \\
\text { purpose of the study, the identity of the investigators, the } \\
\text { time investment for the survey and that completing the } \\
\text { data was anonymous. }\end{array}$ \\
\hline & Data protection & $\begin{array}{l}\text { The following commercial web survey provider was } \\
\text { used: www.surveymonkey.com. An e-mail link to the } \\
\text { survey was created. E-mail addresses of respondents } \\
\text { were automatically stored by the survey provider, for } \\
\text { the purpose of sending reminder messages to non- } \\
\text { responders. E-mail addresses could not be linked to } \\
\text { individual surveys, ensuring anonymity of participants. } \\
\text { The survey provider account is only accessible to the } \\
\text { main investigator, for data protection purposes. }\end{array}$ \\
\hline $\begin{array}{l}\text { Development and pre- } \\
\text { testing }\end{array}$ & $\begin{array}{l}\text { Development and } \\
\text { testing }\end{array}$ & $\begin{array}{l}\text { A non-validated anonymous electronic questionnaire } \\
\text { was designed by the authors. The questions were } \\
\text { composed by an expert panel of four gynaecological } \\
\text { oncologists with experience in colposcopy and treatment } \\
\text { of VAIN/CIN. The web survey was tested before the start } \\
\text { of the study by colleagues. }\end{array}$ \\
\hline \multirow{3}{*}{$\begin{array}{l}\text { Recruitment process and } \\
\text { description of the sample } \\
\text { having access to the } \\
\text { questionnaire }\end{array}$} & $\begin{array}{l}\text { Open survey versus } \\
\text { closed survey }\end{array}$ & $\begin{array}{l}\text { The survey was closed: participants could only complete } \\
\text { the survey through a link in the invitation e-mail. }\end{array}$ \\
\hline & Contact mode & $\begin{array}{l}\text { The target population was contacted by e-mail. A second } \\
\text { and third invitation was sent after three and six weeks. }\end{array}$ \\
\hline & $\begin{array}{l}\text { Advertising the } \\
\text { survey }\end{array}$ & No advertising was used. \\
\hline
\end{tabular}




\begin{tabular}{|c|c|c|}
\hline \multirow[t]{11}{*}{ Survey administration } & Web/E-mail & $\begin{array}{l}\text { The survey was web-based and could be accessed } \\
\text { through a link in an invitation e-mail. The survey tool } \\
\text { included an automatic data collection tool. }\end{array}$ \\
\hline & Context & Not applicable. \\
\hline & $\begin{array}{l}\text { Mandatory/ } \\
\text { voluntary }\end{array}$ & Responding to the survey was voluntary. \\
\hline & Incentives & None \\
\hline & Time/Date & The survey was conducted from Nov 2014 to Jan 2015. \\
\hline & $\begin{array}{l}\text { Randomization } \\
\text { of items or } \\
\text { questionnaires } \\
\end{array}$ & No items or questionnaires were randomized. \\
\hline & \begin{tabular}{|l} 
Adaptive \\
questioning
\end{tabular} & $\begin{array}{l}\text { Adaptive questioning was used twice: the questions on } \\
\text { imiquimod use in VAIN and CIN were only shown to } \\
\text { those participants that actually applied imiquimod. }\end{array}$ \\
\hline & Number of Items & $\begin{array}{l}\text { The full survey consisted of } 37 \text { items. If the participant } \\
\text { never applied imiquimod in CIN or VAIN (the expected } \\
\text { majority of respondents), only } 12 \text { questions were asked. }\end{array}$ \\
\hline & \begin{tabular}{|l|}
$\begin{array}{l}\text { Number of screens } \\
\text { (pages) }\end{array}$ \\
\end{tabular} & $\begin{array}{l}\text { The survey consisted of } 7 \text { pages, with the amount of } \\
\text { questions per page varying from } 2 \text { to } 13 \text {. }\end{array}$ \\
\hline & $\begin{array}{l}\text { Completeness } \\
\text { check }\end{array}$ & $\begin{array}{l}\text { The } 12 \text { questions that were asked to all participants } \\
\text { were all mandatory (this was an automatic option in } \\
\text { the survey programme). The questions that were not } \\
\text { asked to all participants (but only those that applied } \\
\text { imiquimod), were not mandatory as they were not all } \\
\text { applicable in all cases. }\end{array}$ \\
\hline & Review step & $\begin{array}{l}\text { Respondents were able to review and change existing } \\
\text { answers until the survey was finished or until they } \\
\text { closed the survey, through a 'back button'. Reviewing } \\
\text { or changing questions after closing the survey was not } \\
\text { possible. }\end{array}$ \\
\hline
\end{tabular}




\begin{tabular}{|c|c|c|}
\hline \multirow[t]{4}{*}{ Response rates } & Unique site visitor & $\begin{array}{l}\text { All participants could only complete the survey once, } \\
\text { through the link in their e-mail address. Reminders were } \\
\text { only sent to those participants that had not responded } \\
\text { yet, preventing duplicates. }\end{array}$ \\
\hline & \begin{tabular}{|l|} 
View rate (Ratio \\
of unique survey \\
visitors/unique site \\
visitors)
\end{tabular} & Not applicable. \\
\hline & $\begin{array}{l}\text { Participation rate } \\
\text { (Ratio of unique } \\
\text { visitors who agreed } \\
\text { to participate/ } \\
\text { unique first survey } \\
\text { page visitors) }\end{array}$ & The response rate of the study is: $45 \%(79 / 176)$. \\
\hline & \begin{tabular}{|l|} 
Completion rate \\
(Ratio of users who \\
finished the survey/ \\
users who agreed to \\
participate)
\end{tabular} & $\begin{array}{l}\text { Two surveys were incomplete: they were closed before } \\
\text { completion. The completion rate of the study is: } 97 \% \\
\text { (77/79). }\end{array}$ \\
\hline \multirow[t]{4}{*}{$\begin{array}{l}\text { Preventing multiple } \\
\text { entries from the same } \\
\text { individual }\end{array}$} & Cookies used & $\begin{array}{l}\text { Not used, preventing multiple entries from the same } \\
\text { individual was prevented by the electronic survey system, } \\
\text { through e-mail addresses. }\end{array}$ \\
\hline & IP check & $\begin{array}{l}\text { Not used, preventing multiple entries from the same } \\
\text { individual was prevented by the electronic survey system, } \\
\text { through e-mail addresses. }\end{array}$ \\
\hline & Log file analysis & $\begin{array}{l}\text { Not used, preventing multiple entries from the same } \\
\text { individual was prevented by the electronic survey system, } \\
\text { through e-mail addresses. }\end{array}$ \\
\hline & Registration & $\begin{array}{l}\text { Not used, preventing multiple entries from the same } \\
\text { individual was prevented by the electronic survey system, } \\
\text { through e-mail addresses. }\end{array}$ \\
\hline \multirow[t]{3}{*}{ Analysis } & $\begin{array}{l}\text { Handling of } \\
\text { incomplete } \\
\text { questionnaires } \\
\end{array}$ & $\begin{array}{l}\text { Two surveys were incomplete: they were completely } \\
\text { blank. These two surveys were omitted from the results. }\end{array}$ \\
\hline & $\begin{array}{l}\text { Questionnaires } \\
\text { submitted with an } \\
\text { atypical timestamp }\end{array}$ & Not applicable \\
\hline & $\begin{array}{l}\text { Statistical } \\
\text { correction }\end{array}$ & Not applicable \\
\hline
\end{tabular}




\section{Supplemental digital content 2. Questionnaire}

1. What is your professional function?

- Cynecologic oncologist or fellow gynecologic oncology

- Cynecologist with semi-specialization in gynecologic oncology

- Gynecologist with other (semi-) specialization

2. In what hospital type are you currently employed?

- University hospital or specialized oncological center

- Semi-specialized teaching hospitals

. Non-teaching hospital

3. How many colposcopies do you conduct or supervise yearly?

- Less than 30

- 30-100

. More than 100

4. Which method do you usually apply for suspected high-grade dysplasia during colposcopy?

- See-and-treat, regardless of age or future pregnancy wish

- See-and-treat in older patients without future pregnancy wish and diagnostic biopsies in younger patients with potential pregnancy wish

- Diagnostic biopsies, regardless of age or future pregnancy wish

- Other: ...

5. How large do you consider the additional risk of future premature birth following LLETZ, compared to the general population?

Not increased or marginally increased

- Increased with a factor:... (whole number)

6. Do you discuss the additional risk of premature birth in pregnancies following LLETZ, with patients who have an indication for LLETZ?
. Yes
. No
. Other:...

7. Are you aware of the application of imiquimod (Aldara $\left.{ }^{\circledR}\right)$ for genital warts, in literature or clinical practice? Have you ever applied imiquimod for this indication?

- I am aware of this, I have not applied it myself.

. I I am aware of this, I have applied it myself.

. I lam not aware of this.

8. Are you aware of the application of imiquimod (Aldara $\left.{ }^{\circledR}\right)$ for vulvar intraepithelial neoplasia (VIN), in literature or clinical practice? Have you ever applied imiquimod for this indication?

I am aware of this, I have not applied it myself.

- I am aware of this, I have applied it myself.

- I am not aware of this. 
9. Are you aware of the application of imiquimod (Aldara ${ }^{\circledR}$ ) for vaginal intraepithelial neoplasia (VAIN), in literature or clinical practice? Have you ever applied imiquimod for this indication?

I am aware of this, I have not applied it myself. (to question 22)

I am aware of this, I have applied it myself. (to question 10)

I am not aware of this. (to question 22)

10. You have applied imiquimod in VAIN. How often have you applied it for this indication?

- 1-5 times

- 6-10 times

. More than 10 times

11. What was(/were) the lesion grade(s)?

Low grade lesion(s) (VAIN 1$)$

High grade lesion(s) (VAIN 2-3)

Both

12. Was it a primary or recurrent lesion?

Primary lesion(s)

Recurrent lesion(s)

- Both

13. What was your rationale for using imiquimod in the treatment of this lesion? (open question)

14. How did you apply the imiquimod? (multiple options possible)

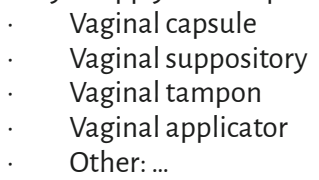

15. What was the dosing scheme and treatment period? (open question)

16. How did you evaluate treatment efficacy (multiple options possible)

- Cytology only

- Colposcopy without biopsies

- Colposcopy with biopsies

- Other: ...

17. Was treatment of the VAIN lesion(s) with imiquimod successful? (multiple options possible)

- Yes: lesions regression, invasive treatment no longer necessary

- Yes: complete remission, invasive treatment no longer necessary

- No: persistent lesion

. No: progressive lesion

18. Which side effects did you notice in the patients who were treated with imiquimod for VAIN? (multiple options possible)
. Vaginal/vulvar pain
- Vulvar erythema
- Vulvar erosion/ulceration
- Vaginal discharge
- Flu-like symptoms
. Other:... 
19. How serious were the side effects mentioned in the last questions, in general?

. No side effects noticed

- Mild side effects

- Moderate side effects

- Severe side effects

. Comment: ...

20. Did any of your patients stop imiquimod treatment due to side effects?

- Yes

- No

. Comment: ...

21. Would you recommend treatment of VAIN with imiquimod and would you apply it again?

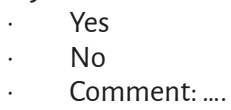

22. Are you aware of the application of imiquimod (Aldara $\left.{ }^{\circledR}\right)$ for cervical intraepithelial neoplasia $(C I N)$, in literature or clinical practice? Have you ever applied imiquimod for this indication?

- I I am aware of this, I have not applied it myself. (to question 36)

- I I am aware of this, I have applied it myself. (to question 23)

. I I am not aware of this. (to question 36)

23. You have applied imiquimod in $\mathrm{CIN}$. How often have you applied it for this indication?

$$
\begin{array}{ll}
\text {. } & \text { 1-5 times } \\
\text {. } & \text { More than } 10 \text { times }
\end{array}
$$

24. What was(/were) the lesion grade(s)?

$$
\begin{array}{ll}
. & \text { Low grade lesion(s) (CIN 1) } \\
\text {. } & \text { High grade lesion(s) (CIN 2-3) } \\
& \text { Both }
\end{array}
$$

25. Was it a primary or recurrent lesion?

$$
\begin{array}{ll}
\text {. } & \text { Primary lesion(s) } \\
\text {. } & \text { Recurrent lesion(s) } \\
\text { Both }
\end{array}
$$

26. What was your rationale for using imiquimod in the treatment of this lesion? (open question)

27. How did you apply the imiquimod? (multiple options possible)

$$
\begin{array}{ll}
\text {. } & \text { Vaginal capsule } \\
\text {. } & \text { Vaginal suppository } \\
\text {. } & \text { Vaginal applicator } \\
\text {. } & \text { Other:... }
\end{array}
$$

28. What was the dosing scheme and treatment period? (open question) 
29. How did you evaluate treatment efficacy (multiple options possible)

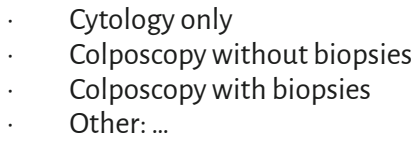

30. Was treatment of the CIN lesion(s) with imiquimod successful? (multiple options possible)

- Yes: lesions regression, invasive treatment no longer necessary

- Yes: complete remission, invasive treatment no longer necessary

- No: persistent lesion

. No: progressive lesion

31. Which side effects did you notice in the patients who were treated with imiquimod for CIN? (multiple options possible)
- Vaginal/vulvar pain
. Vulvar erythema
- Vulvar erosion/ulceration
- Vaginal discharge
- Flu-like symptoms
. Other: ...

32. How serious were the side effects mentioned in the last questions, in general?
- No side effects noticed
- Mild side effects
- Moderate side effects
- Severe side effects
. Comment: ...

33. Did any of your patients stop imiquimod treatment due to side effects?

$$
\begin{array}{ll}
\text {. } & \text { Yes } \\
\text {. } & \text { No } \\
& \text { Comment: ... }
\end{array}
$$

34. Would you recommend treatment of CIN with imiquimod and would you apply it again?

$$
\begin{array}{ll}
\text {. } & \text { Yes } \\
\text {. } & \text { No } \\
\text { Comment:.... }
\end{array}
$$

35. We wish to conduct a retrospective analysis of treatment of (recurrent) CIN lesions with imiquimod. Are you willing to share more detailed data with us? If you agree, you can leave your contact details here. We will not use your contact details for the analysis of this questionnaire.

$$
\text { Name, e-mail address and/or telephone number }
$$


36. Recently, an RCT was published on the treatment efficacy of imiquimod in high-grade CIN (Grimm, Obstet Gynecol, 2012). 59 patients with high-grade CIN (CIN2-3) were randomized to receive imiquimod or placebo during 16 weeks. Disease regression and remission was significantly more common in patients who were treated with imiquimod than in patients treated with placebo $(73 \%$ vs $39 \%$ and $47 \%$ vs $14 \%$ respectively). There was no disease progression in the imiquimod group. Side effects were acceptable for all patients.

If these results were to be confirmed in a larger trial, would you then consider using imiquimod in the treatment of high-grade $\mathrm{CIN}$ (CIN 2-3), for example in younger patients with a future pregnancy wish?

$$
\begin{array}{ll}
\text {. } & \text { Yes } \\
\text {. } & \text { No } \\
\text {. } & \text { Comment: ... }
\end{array}
$$

37. Would you consider participating in a randomized controlled trial, evaluating treatment efficacy of imiquimod in treatment of high-grade CIN?

$$
\begin{array}{ll}
\text {. } & \text { Yes } \\
\text {. } & \text { No } \\
\text {. } & \text { Comment in case of 'maybe' or 'no':... }
\end{array}
$$





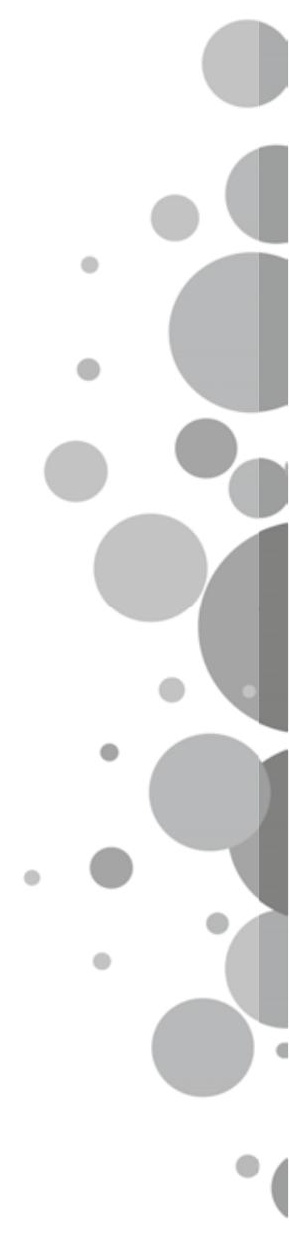




\section{CHAPTER 8}

Treatment of cervical intraepithelial neoplasia: patients preferences for surgery orimmunotherapy with imiquimod

Margot M Koeneman, Brigitte A Essers, Cees C Cerestein, Anna JM van de Sande, Rogier JNTM Litjens, Dieuwke Boskamp, Medi F] Coossens,

Heleen ] van Beekhuizen, Roy FPM Kruitwagen, Arnold] Kruse, Carmen D Dirksen

Journal of Immunotherapy. 2017; 40:148-153 


\section{Abstract}

Objective: Imiquimod has been studied as a non-invasive pharmacological treatment alternative to large loop excision of the transformation zone (LLETZ) for high-grade cervical intraepithelial neoplasia $(\mathrm{CIN})$ in order to prevent long-term obstetric complications from surgical treatment. This study aims to investigate womens' preferences for treatment of high-grade CIN with imiquimod or LLETZ.

Methods: A labelled discrete choice experiment was conducted among 100 women with abnormal cervical cytology in five hospitals in the Netherlands between March 2014 and December 2015. Participants were asked to choose between imiquimod treatment or standard surgical treatment in nine separate scenarios, based on the following treatment characteristics: treatment success rate, rate of side effects, risk of premature birth in subsequent pregnancies, and risk of subfertility after treatment. The levels of these characteristics differed for the imiquimod alternatives.

Results: Women assigned a positive utility to LLETZ compared to imiquimod. When making a choice for imiquimod, women preferred a higher treatment success rate and a lower risk of premature birth, infertility, and side effects. The choice for imiquimod treatment was also influenced by the intention of a future pregnancy. Subgroup analyses revealed that a lower efficacy regarding imiquimod might be more acceptable for women who desired a future pregnancy compared to women who did not desire a future pregnancy.

Conclusion: Women with a future pregnancy wish may prefer treatment of high-grade CIN with imiquimod cream over LLETZ, if the risk of subfertility and premature birth is low. 


\section{Introduction}

High-grade cervical intraepithelial neoplasia $(\mathrm{CIN})$ is a relatively common condition and is considered to be the precursor of cervical cancer. It is usually caused by Human Papillomavirus (HPV)-infection of the uterine cervix.[1] Currently, the gold-standard treatment for high-grade $\mathrm{CIN}$ is surgical excision, which is usually performed by large loop excision of the transformation zone (LLETZ). Common side effects of LLETZ are postoperative haemorrhage and vaginal discharge. More importantly, LLETZ is associated with potentially serious long-term side effects. An approximately twofold increase in premature birth is seen in pregnancies after a LLETZ procedure. $[2,3]$ Recent evidence also suggests an increase in subfertility after LLETZ. A casecontrol study showed that women with a history of cervical treatment for $\mathrm{CIN}$ are at increased risk of subfertility defined as a time to conception of more than 12 months. [4]

Imiquimod cream (Aldara) has been studied as a non-invasive pharmacological treatment alternative in order to reduce pregnancy-related side effects of LLETZ.[5-7] Imiquimod is a toll-like receptor antagonist with antiviral and antitumor properties. It is not currently registered for the treatment of high-grade $\mathrm{CIN}$ and is not part of treatment guidelines, but it is widely used in HPVrelated vulvar intraepithelial neoplasia (VIN) with good results. [7] The rationale of imiquimod treatment of HPV-induced lesions is to enhance the antiviral immunity of those who are unable to clear HPV naturally.

Treatment efficacy of imiquimod in high-grade $\mathrm{CIN}$ has been studied in one randomized controlled trial RCT and shows promising results: treatment efficacy was reported in $73 \%$ of subjects compared to $39 \%$ in the placebo group.[5] In comparison, treatment efficacy of LLETZ treatment is higher, with $95 \%$ of patients being adequately treated.[8] Vaginal imiquimod can be self-administered by patients, but the treatment is time-consuming and side effects are common. Frequently reported side effects include vaginal pruritus and/or pain, vaginal discharge, and flu-like symptoms. [5, 9] Imiquimod, therefore, does not seem to qualify as a replacement for surgical treatment in all women. Nevertheless, subgroups may be identified for which imiquimod treatment does provide a good alternative. Our previous study has shown that gynecologists consider women who desire a future pregnancy and women with recurrent lesions as potential candidates for imiquimod treatment.[10]

The choice for either surgical treatment or pharmacological treatment with imiquimod for highgrade CIN is likely to be preference-sensitive. However, patients' preferences for these treatment modalities are currently unknown. Knowledge of patients' preferences and explanations for their preference may further aid the identification of subgroups of women for whom imiquimod treatment can be considered a good alternative to LLETZ treatment. The current study investigated patients' preferences for either imiquimod or LLETZ in the treatment of high-grade CIN by means of a discrete choice experiment. 


\section{Methods}

\section{Participants}

The study was performed in five Dutch hospitals. The target population consisted of women diagnosed with premalignant abnormal cervical cytology (PAP 2/ASCUS to PAP 3b/HSIL), for whom colposcopy was planned and who would thus potentially need treatment for high-grade CIN.

\section{Discrete choice experiment}

Patient preferences were investigated with a discrete choice experiment (DCE).[11] In a DCE, two or more interventions are compared based on the attributes of these specific interventions. Attributes represent the characteristics of the intervention, such as treatment efficacy or side effects. The preference for either intervention is then based on the levels of the different attributes: the rate of successful treatment or the rate of side effects. Respondents choose between different hypothetical scenarios described in terms of their attributes and associated levels. Several choice sets are presented that consist of two or more scenarios in which the levels of the attributes differ. The assumption is that a respondent will choose the scenario that provides the highest utility or benefit.

\section{Attributes and levels}

Selection of the most relevant attributes and their levels was based on literature research followed by a systematic discussion with members of the research team, consisting of a gynecologist (AK), a resident-gynecologist (MK), and two DCE experts (CD and BE). Three reviews and three clinical trials reporting on outcome measures of LLETZ treatment and vaginal imiquimod treatment were identified. $[3-5,8,9,12]$ Subsequently, six interviews were conducted with women who had undergone colposcopy (with or without diagnostic biopsies or LLETZ treatment) to verify the relevance and relative importance of the selected attributes. The interviews consisted of qualitative, open-ended questions and discrete interview questions. Ages differed from 30 to 55 , and three women desired a future pregnancy. The patient interviews did not lead to the identification of additional attributes. The selected attributes and their levels are illustrated in Table 1. Because the evidence on the attributes and levels of imiquimod treatment is limited, we designed a DCE with two hypothetical imiquimod options per choice set, in which levels were varied.

\section{Study procedures}

An efficient main-effect design was created, using Ngene software, to select a subset of all possible choice sets. [13] An efficient design refers to the precision with which the different model parameters can be estimated.[14] Prior parameter values were based on the importance ranking that was provided in patient interviews. Based on the S-estimate (sample size) of the efficient design, 50 patients were sufficient to estimate the significant parameters for a main-effect model. We aimed for the inclusion of 100 patients in order to be able to explore potential interaction effects. 
Table 1. Attributes and levels used in the discrete choice experiment

\begin{tabular}{|c|c|c|c|c|}
\hline Attribute & Explanation of attribute & $\begin{array}{l}\text { Levels of the } \\
\text { attribute } \\
\text { for LLETZ } \\
\text { treatment }\end{array}$ & $\begin{array}{l}\text { Levels of the } \\
\text { attribute for } \\
\text { imiquimod } \\
\text { treatment }\end{array}$ & Ref. \\
\hline $\begin{array}{l}\text { Chance of successful } \\
\text { treatment }\end{array}$ & $\begin{array}{l}\text { Treatment is successful when the entire } \\
\text { CIN lesion has resolved after treatment } \\
\text { and no additional treatment is necessary. }\end{array}$ & $95 \%$ & $55 \%, 75 \%, 95 \%$ & $5,8,12$ \\
\hline $\begin{array}{l}\text { Risk of premature } \\
\text { birth after } \\
\text { treatment }\end{array}$ & $\begin{array}{l}\text { Premature birth is the birth of a baby } \\
\text { before } 37 \text { weeks of gestation. Premature } \\
\text { babies often have to be admitted to a } \\
\text { neonatal ward and experience more health } \\
\text { problems. }\end{array}$ & $12 \%$ & $6 \%, 12 \%$ & 2,3 \\
\hline $\begin{array}{l}\text { Risk of subfertility } \\
\text { after treatment }\end{array}$ & $\begin{array}{l}\text { Subfertility is when the time to conception } \\
\text { is longer than } 12 \text { months. }\end{array}$ & $16 \%$ & $8 \%, 16 \%$ & 4 \\
\hline \multicolumn{5}{|l|}{ Side effects" } \\
\hline $\begin{array}{l}\text { 1. Vaginal pruritus/ } \\
\text { pain }\end{array}$ & $\begin{array}{l}\text { Irritation of the vaginal mucosa, which can } \\
\text { lead to pruritus or pain. }\end{array}$ & NA & $10 \%, 25 \%$ & 5 \\
\hline 2. Abdominal pain & Pain in the lower abdomen. & $15 \%$ & NA & 12 \\
\hline 3. Vaginal discharge & Bloody or white vaginal discharge. & $20 \%$ & $10 \%, 30 \%$ & 9,12 \\
\hline 4. Vaginal bleeding & $\begin{array}{l}\text { Vaginal bleeding can be both brown and } \\
\text { red blood loss. }\end{array}$ & $25 \%$ & NA & 12 \\
\hline $\begin{array}{l}\text { 5. Flu-like } \\
\text { symptoms }\end{array}$ & $\begin{array}{l}\text { Flu-like symptoms include myalgia, } \\
\text { headache and fever. }\end{array}$ & NA & $10 \%, 25 \%$ & 5,9 \\
\hline
\end{tabular}

* study subjects were informed that the presented levels indicated the levels of moderate to severe complaints, during the first two weeks after LLETZ treatment and during the full period of imiquimod treatment.

$\mathrm{CIN}=$ cervical intraepithelial neoplasia; LLETZ = large loop excision of the transformation zone; NA = not applicable

In total, nine choice sets were created (figure 1). The ordering of the attributes was varied over four versions of the questionnaire to control for a potential attribute-ordering effect. To test internal consistency, choice set 5 was repeated at the end of the questionnaire (choice set 10). Internal consistency was evaluated by Cohen's kappa, in which a kappa value $<0.20$ denotes poor agreement, $0.21-0.40$ fair, $0.41-0.60$ moderate, $0.60-0.80$ good, and $0.81-1.00$ very good agreement.[15] 


Question 1

\begin{tabular}{|c|c|c|c|}
\hline Attribute & $\begin{array}{l}\text { Imiquimod } \\
\text { option } 1\end{array}$ & $\begin{array}{l}\text { Imiquimod } \\
\text { option } 2\end{array}$ & LLETZ \\
\hline Chance of successful treatment & $\begin{array}{l}55 \% \\
55 \text { of } 100 \text { women }\end{array}$ & $\begin{array}{l}95 \% \\
95 \text { of } 100 \text { women }\end{array}$ & $\begin{array}{l}95 \% \\
95 \text { of } 100 \text { women }\end{array}$ \\
\hline Risk of premature birth & $\begin{array}{l}12 \% \\
12 \text { of } 100 \text { women }\end{array}$ & $\begin{array}{l}6 \% \\
6 \text { of } 100 \text { women }\end{array}$ & $\begin{array}{l}12 \% \\
12 \text { of } 100 \text { women }\end{array}$ \\
\hline Risk of subfertility & $\begin{array}{l}8 \% \\
8 \text { of } 100 \text { women }\end{array}$ & $\begin{array}{l}16 \% \\
16 \text { of } 100 \text { women }\end{array}$ & $\begin{array}{l}16 \% \\
16 \text { of } 100 \text { women }\end{array}$ \\
\hline \multicolumn{4}{|l|}{ Risk of side effects: } \\
\hline Vaginal pruritus or pain & $\begin{array}{l}10 \% \\
10 \text { of } 100 \text { women }\end{array}$ & $\begin{array}{l}25 \% \\
25 \text { of } 100 \text { women }\end{array}$ & - \\
\hline Abdominal pain & - & - & $\begin{array}{l}15 \% \\
15 \text { of } 100 \text { women }\end{array}$ \\
\hline Vaginal discharge & $\begin{array}{l}10 \% \\
10 \text { of } 100 \text { women }\end{array}$ & $\begin{array}{l}30 \% \\
30 \text { of } 100 \text { women }\end{array}$ & $\begin{array}{l}20 \% \\
20 \text { of } 100 \text { women }\end{array}$ \\
\hline Vaginal bleeding & - & - & $\begin{array}{l}25 \% \\
25 \text { of } 100 \text { women }\end{array}$ \\
\hline Flu-like symptoms & $\begin{array}{l}10 \% \\
10 \text { of } 100 \text { women }\end{array}$ & $\begin{array}{l}\mathbf{2 5} \% \\
25 \text { of } 100 \text { women }\end{array}$ & - \\
\hline
\end{tabular}

\begin{tabular}{|l|l|l|l|l|}
\hline \multirow{2}{*}{ Which treatment do you prefer? } & $\begin{array}{l}\text { Imiquimod } \\
\text { option 1 }\end{array}$ & $\begin{array}{l}\text { Imiquimod } \\
\text { option 2 }\end{array}$ & LLETZ \\
\hline
\end{tabular}

\section{Figure 1. Example of a choice set}

Ten of such choice sets were presented. Study subjects were asked to choose one treatment alternative in each choice set: either imiquimod option 1, imiquimod option 2 or LLETZ.

A pilot study was conducted in ten women for feasibility, understanding, and to check whether women were willing to trade (switch between the two treatment options according to different characteristics). Based on oral feedback by these women, minor adjustments were made to the explanatory sections on high-grade CIN, LLETZ, and imiquimod treatment.

After giving informed consent, participants received written information and the DCE questionnaire. The information consisted of a general explanation of the DCE experiment, information about high-grade $\mathrm{CIN}$, and a detailed description of both LLETZ and imiquimod treatment, including illustrations on the LLETZ procedure and the insertion procedure of imiquimod cream and a table with the selected attributes and their levels. The description of 
LLETZ was based on the existing local information brochure, the description of imiquimod treatment was based on the treatment protocol according to the RCT by Grimm et al.[5] All questionnaires were completed before the planned colposcopy.

\section{Data analysis}

The respondents' choices were analyzed with a multinomial logit regression model in Nlogit version 5 . The following model was estimated:

$\begin{aligned} \mathrm{V}(\text { imiquimod })= & \beta_{0}+\left(\beta_{1}^{*} \text { treatment success }\right) \\ & \left.+\left(\beta_{2}^{*} \text { premature birth }\right)+{ }^{*} \beta_{3}^{*} \text { infertility }\right) \\ & +\left(\beta_{4}^{*} \text { pain }\right)+\left(\beta_{5}{ }^{*} \text { discharge }\right)+\left(\beta_{6}{ }^{*} \text { flu }\right) \\ \mathrm{V}(\text { LLETZ treatment }) \quad= & \left.\beta_{7} \text { (fixed constant }\right)\end{aligned}$

where $V$ represents the relative utility score patients derive from a treatment with imiquimod or LLETZ treatment; $\beta_{0}$ is a constant that, if positive, reflects a preference for the label imiquimod; $\beta_{1}$ to $\beta_{6}$ are the coefficients reflecting the impact of a particular attribute level on the utility score; $\beta_{7}$ is the coefficient (i.e., alternative specific constant) for the LLETZ treatment with fixed levels. The sign of a coefficient shows whether an attribute has a positive or negative impact on utility. A relative utility score can be calculated by filling in the regression equation with estimated coefficients from the multinomial logit model and levels of the attributes. A higher utility score means that patients prefer one treatment over another. To examine whether preferences for the attributes "risk of subfertility", "risk of premature birth in subsequent pregnancy," and the fixed alternative LLETZ-treatment are different depending on a desire for a future pregnancy, we included these interaction terms in the model. In addition, a subgroup analysis was performed based on women with or without a future pregnancy wish.

\section{Ethics approval}

The study was evaluated and approved of by the Medical Ethics Committee of the Maastricht University Medical Center (MUMC/METC 13-4-116, 6 January 2014). All study procedures were conducted according to the Declaration of Helsinki, $7^{\text {th }}$ revision, 2013.

\section{Results}

\section{Respondents and general results}

Respondents were recruited in five Dutch hospitals between March 2014 and December 2015. A total of 177 women were consecutively approached for participation in the study, of which 100 gave informed consent and completed the questionnaire (response rate 56.5\%). Baseline characteristics of the respondents can be found in Table 2. Three questionnaires had missing data (six questions in total). Of the 100 women, 82 evaluated the questions as clear or very clear. The internal consistency of the respondents was good, as reflected by a kappa value of 0.79 ( $p$-value $<0.01)$. 
Table 2. Baseline characteristics of the respondents

\begin{tabular}{cl}
\hline Characteristic & $\mathbf{N}=\mathbf{1 0 0}$ \\
\hline Age in years (mean, range) & $38.5,21-70$ \\
Degree of education (absolute) & \\
- Secondary education or intermediate vocational education & 62 \\
- Advanced vocational school or university & 35 \\
Parity & 3 \\
- Nulliparous & \\
- Primiparous & 39 \\
- Multiparous & 23 \\
- Unknown & 35 \\
Age in years at first birth (mean, range) & 3 \\
Pregnancy wish in the future & $27,18-36$ \\
- Yes & \\
- No & 34 \\
- Unsure & 53 \\
- Unknown & 9 \\
Previous colposcopy & 4 \\
- Yes & \\
- No & 77 \\
- Unknown & 21 \\
Previous LLETZ treatment or conization & 2 \\
- Yes & \\
- No & 18 \\
- Unknown & 81 \\
\hline
\end{tabular}

LLETZ $=$ Large loop excision of the transformation zone.

\section{DCE results: Main-effect model}

The results of the main-effect multinomial logit regression model are shown in Table 3. Women derived a positive utility from choosing the standard LLETZ treatment, as shown by the significant positive coefficient of 4.25 . All attributes representing imiquimod treatment were considered important, and women significantly preferred a higher treatment success rate and a lower risk of premature birth, infertility, and side effects. A higher utility score for imiquimod treatment compared to LLETZ was only reached when the success rate is $80 \%$ with a low risk of subfertility and premature birth in subsequent pregnancies and low levels of side effects (utility score 4.4: $\mathrm{V}($ imiquimod $)=(0.08 * 80 \%$ treatment success $)+(-0.13 * 6 \%$ premature birth $)+\left(-0.11^{*} 8 \%\right.$ infertility $)+(-0.03 * 10 \%$ vaginal pain $)+\left(-0.013^{*} 10 \%\right.$ vaginal discharge $)+(-0.029 * 10 \%$ flu $\left.)\right)$. In the case of a low risk of subfertility and premature birth (as is expected), but a high risk of side effects, treatment efficacy should reach $95 \%$ in order to provide a higher utility score compared to LLETZ treatment.

\section{DCE results: Interaction model}

The results of the interaction model (2) are presented in Table 3. All three interactions were statistically significant, indicating that preferences for the attributes "risk of subfertility", "risk 
of premature birth in subsequent pregnancy," and the fixed alternative LLETZ treatment are significantly different depending on a woman's plans for a future pregnancy. The LLETZ treatment provides a significantly higher benefit for women without a pregnancy wish compared to women with a pregnancy wish. The model 2 , which includes the interactions, significantly improves the model fit compared to model 1 (likelihood ratio test $\chi^{2}(251) \geq \chi^{2}(7.81) ; p<0.05$ ).

Table 3. Results of the multinomial model

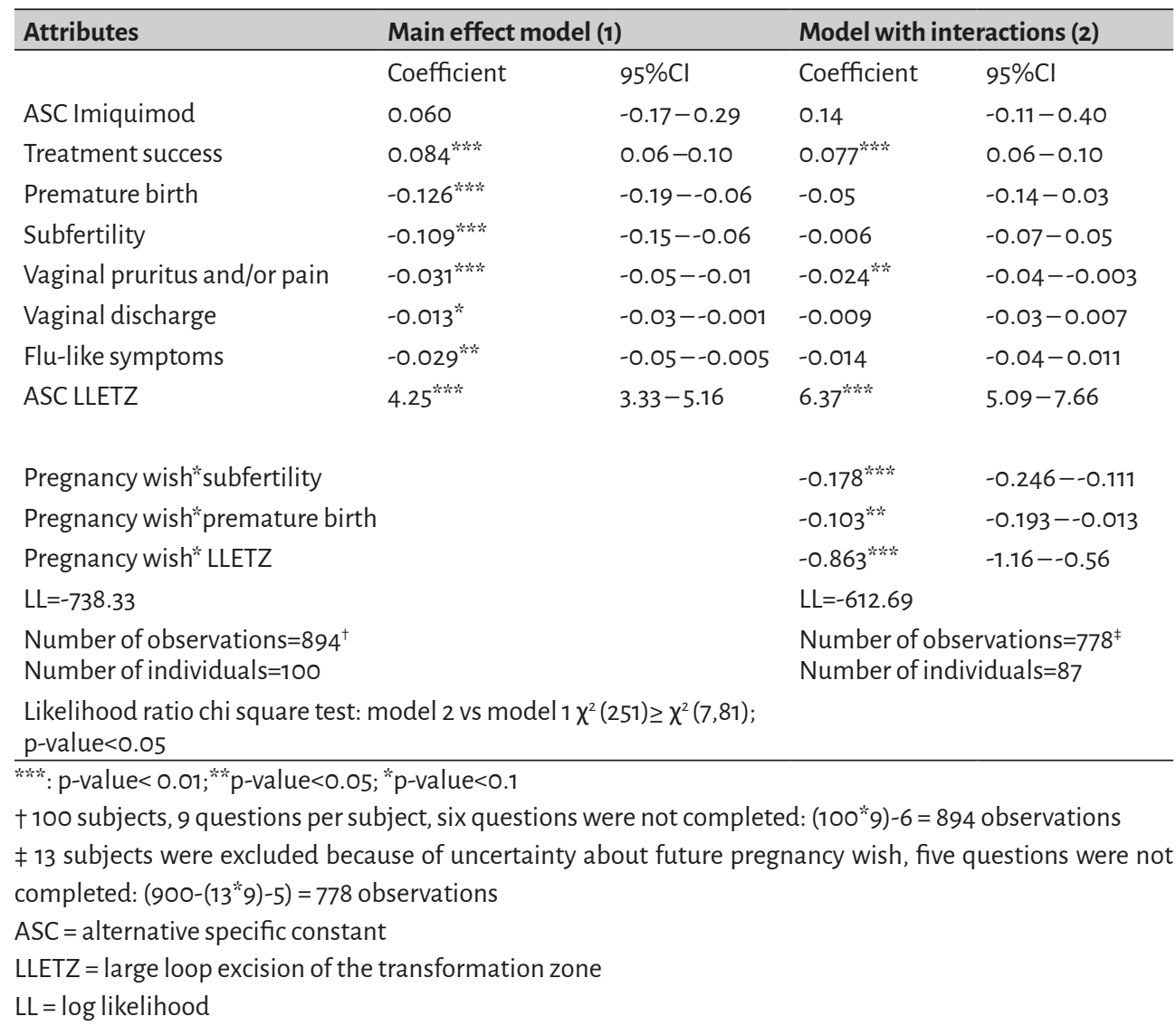

DCE results: Subgroup analysis of women with and without the desire for a future pregnancy

Table 4 presents the results for women with a future pregnancy wish and without a pregnancy wish. Women with a future pregnancy wish show a significant preference for a higher chance of treatment success and a lower risk of subfertility and premature birth. The other attributes do not play a role in their decision for imiquimod treatment. They also assign a positive utility (3.35) to the LLETZ treatment, although this value is significantly lower compared to women who do not desire a future pregnancy (5.76). For women who are planning a future pregnancy, imiquimod must have a treatment success of at least around $72 \%$ (0.08*72\%) with low levels for subfertility 
$(-0.19 * 8 \%)$ and premature birth $(-0.15 * 6 \%)$ in order to result in a higher utility score than that for LLETZ treatment.

Table 4. Subgroup analysis

\begin{tabular}{lllll}
\hline Attributes & \multicolumn{2}{l}{$\begin{array}{l}\text { Women with desire for future } \\
\text { pregnancy }\end{array}$} & \multicolumn{2}{l}{$\begin{array}{l}\text { Women without desire for } \\
\text { future pregnancy }\end{array}$} \\
\hline & Coefficient & $95 \% \mathrm{Cl}$ & Coefficient & $95 \% \mathrm{Cl}$ \\
ASC_Imiquimod & 0.173 & $-0.21-.0 .56$ & 0.118 & $-0.23-0.46$ \\
Treatment success & $0.081^{* * * *}$ & $0.05-0.11$ & $0.074^{* * * *}$ & $0.048-0.09$ \\
Premature birth & $-0.156^{* * * *}$ & $-0.25--0.05$ & -0.054 & $-0.16-0.03$ \\
Subfertility & $-0.190^{* * * *}$ & $-0.26--0.11$ & -0.008 & $-0.08-0.06$ \\
Vaginal pruritus and/or pain & -0.022 & $-0.05-0.01$ & $-0.027^{* *}$ & $-0.055-0.0008$ \\
Vaginal discharge & -0.004 & $-0.03-0.020$ & -0.013 & $-0.035-0.008$ \\
Flu-like symptoms & -0.006 & $-0.04-0.03$ & -0.022 & $-0.058-0.013$ \\
ASC_LLETZ-treatment & $3.35^{* * * *}$ & $1.84-4.86$ & $5.76^{* * * *}$ & $4.28-7.23$ \\
& $\mathrm{~N} .0 \mathrm{bs}=305$ & & $\mathrm{~N} .0 \mathrm{bs}=473$ & \\
& $\mathrm{~N} . \mathrm{ind}=34$ & & $\mathrm{~N} . \mathrm{ind}=53$ & \\
\hline
\end{tabular}

**** p-value $<0.01 ; *$ p-value $<0.05$, "p-value $<0.1 ;$

$\mathrm{ASC}=$ alternative specific constant

LLETZ = large loop excision of the transformation zone

\section{Discussion}

This is the first study reporting on patient preferences in the choice between imiquimod and LLETZ as treatment modalities for high-grade $\mathrm{CIN}$. The results indicate that women derive a positive utility from the standard LLETZ treatment compared to imiquimod treatment. With respect to imiquimod, women prefer a higher treatment success rate and a lower risk of premature birth, infertility, and side effects. Subgroup analysis showed that for women who planned a future pregnancy, imiquimod might be a more acceptable treatment modality than for women without a pregnancy wish if the risk of subfertility and premature birth are low.

The study shows that the preference for imiquimod treatment compared to LLETZ treatment is influenced by a future pregnancy wish. This is understandable, considering that the main advantage of imiquimod treatment is a reduction in future subfertility and premature birth. These findings correspond with the results of a recent survey among gynecologists concerning their experience and attitude regarding imiquimod treatment of high-grade CIN.[10] This survey showed that off-label application of imiquimod in high-grade $\mathrm{CIN}$ is largely restricted to patients with a future pregnancy wish and/or recurrent lesions in which (repeated) LLETZ treatment may cause long-term morbidity. Our study shows that in women planning a future pregnancy wish, the side effects of vaginal imiquimod treatment may indeed outweigh the risks of surgical treatment, making imiquimod treatment an acceptable treatment alternative for these women. Treatment efficacy is an important attribute in the decision between LLETZ and imiquimod 
treatment. The only RCT on imiquimod treatment of high-grade CIN showed a treatment success rate of $73 \%$ (defined as histologic regression to $\mathrm{CIN} 1$ or less after 20 weeks from the beginning of treatment). Complete histologic regression was seen in $47 \%$ of patients. [5] Assuming a high risk of side effects and a low risk of subfertility and premature birth in clinical practice, our study showed that a treatment efficacy of $95 \%$ for imiquimod treatment is generally desired for women to derive a higher benefit from imiquimod treatment than from LLETZ treatment. This supports our notion that imiquimod therapy will not develop as a treatment alternative for the general population of women with high-grade CIN. However, we also showed that women who planned a future pregnancy would possibly accept a lower treatment success rate if the risk of subfertility and premature birth was low. In that case, a treatment success rate of $72 \%$ would result in women preferring imiquimod over LLETZ treatment. Additional evidence on the long-term treatment efficacy of imiquimod treatment should clarify whether a treatment success rate of at least $72 \%$ is actually achievable. Ideally, the individual probability of treatment success should be predictable. A combination of patient characteristics and biomarkers reflecting host, viral, and cellular factors may provide a model to make the individual response to imiquimod predictable.[16] Patients with higher chances of treatment success could then be identified and counselled based on the results of this study.

An important strength of this study includes the innovative aspect of the study subject: this is the first study on patient preferences regarding the treatment of CIN. It provides the physician with information on relevant attributes in the choice between imiquimod and LLETZ treatment and on subgroups for who a new treatment modality might be indicated. Another strength is the relatively large patient population, which is twice the size of the calculated sample size. The moderate response rate of $56 \%$ can be considered a limitation of this study. Patients received the questionnaire by mail and were asked to complete it before the colposcopy to prevent biased results due to experiences during the colposcopy. Patients who had not completed the questionnaire at the time of the colposcopy could therefore not be included. Nevertheless, the effect of a potential sampling or response bias as a result of low response rates seems limited in other types of surveys. [17] Moreover, the study population is diverse with regard to age and future pregnancy plans, which are characteristics that could be expected to influence their treatment preference. Another potential limitation is the inclusion of the risk of subfertility as a treatment characteristic. A meta-analysis was published after the start of our DCE that concluded that there is no evidence suggesting that treatment for CIN adversely affects fertility. [18] It must be noted, however, that the results of this meta-analysis concerning time to conceive (subfertility) were based on three studies, of which two were more than 20 years old. The only recent study indicates a higher incidence of subfertility in women after LLETZ treatment. [4] Consequently, it cannot be ruled out that the risk of subfertility is higher after LLETZ treatment.

In conclusion, women generally have a preference for LLETZ treatment of high-grade CIN. A preference for imiquimod treatment is influenced by the desire for a future pregnancy, in which case lower treatment success rates might be more acceptable as long as the risk of premature birth and subfertility are low. Additional evidence is necessary to confirm the safety and applicability of imiquimod treatment of high-grade $\mathrm{CIN}$ and should clarify the actual levels of treatment success and side effects. 


\section{References}

1. zur Hausen H. Papillomaviruses causing cancer: evasion from host-cell control in early events in carcinogenesis. Journal of the National Cancer Institute. 2000;92(9):690-8.

2. Crane JM. Pregnancy outcome after loop electrosurgical excision procedure: a systematic review. Obstetrics and gynecology. 2003;102(5 Pt 1):1058-62.

3. Kyrgiou M, Koliopoulos G, Martin-Hirsch P, Arbyn M, Prendiville W, Paraskevaidis E. Obstetric outcomes after conservative treatment for intraepithelial or early invasive cervical lesions: systematic review and meta-analysis. Lancet. 2006;367(9509):489-98.

4. Spracklen CN, Harland KK, Stegmann B], Saftlas AF. Cervical surgery forcervical intraepithelial neoplasia and prolonged time to conception of a live birth: a case-control study. BJOC : an international journal of obstetrics and gynaecology. 2013;120(8):960-5.

5. Grimm C, Polterauer S, Natter C, Rahhal ], Hefler L, Tempfer CB, et al. Treatment of cervical intraepithelial neoplasia with topical imiquimod: a randomized controlled trial. Obstetrics and gynecology. 2012;120(1):152-9.

6. Lin CT, Qiu JT, Wang C], Chang SD, Tang YH, Wu P], et al. Topical imiquimod treatment for human papillomavirus infection in patients with and withoutcervical/vaginal intraepithelial neoplasia. Taiwanese journal of obstetrics \& gynecology. 2012;51(4):533-8.

7. de Witte C], van de Sande A], van Beekhuizen H], Koeneman MM, Kruse A], Gerestein CG. Imiquimod in cervical, vaginal and vulvar intraepithelial neoplasia: A review. Gynecologic oncology. 2015;139(2):377-84.

8. Martin-Hirsch PP, Paraskevaidis E, Bryant A, Dickinson HO. Surgery for cervical intraepithelial neoplasia. The Cochrane database of systematic reviews. 2013;12:CDo01318.

9. Pachman DR, Barton DL, Clayton AC, McGovern RM, Jefferies JA, Novotny PJ, et al. Randomized clinical trial of imiquimod: an adjunct to treating cervical dysplasia. American journal of obstetrics and gynecology. 2012;206(1):42 e1-7.

10. Koeneman MM, van de Sande A], van Beekhuizen H], Gerestein KG, van de Laar R, Kruitwagen RF, et al. Physicians' Awareness, Attitudes, and Experiences Regarding Imiquimod Treatment of Vaginal and Cervical Intraepithelial Neoplasia. Journal of lower genital tract disease. 2016;20(1):75-9.

11. Amaya-amaya M GK, Ryan M. Using Discrete Choice Experiments to Value Health and Health Care. Heidelberg: Springer; 20082008.

12. Group T, Sharp L, Cotton S, Cochran C, Gray N, Little ], et al. After-effects reported by women following colposcopy, cervical biopsies and LLETZ: results from the TOMBOLA trial. BJOG : an international journal of obstetrics and gynaecology. 2009;116(11):1506-14.

13. http://www.choice-metrics.com [accessed 20.10.2013].

14. ReedJohnson F, Lancsar E, Marshall D, Kilambi V, Muhlbacher A, Regier DA, etal. Constructing experimental designs for discrete-choice experiments: report of the ISPOR Conjoint Analysis Experimental Design Cood Research Practices Task Force. Value in health : the journal of the International Society for Pharmacoeconomics and Outcomes Research. 2013;16(1):3-13.

15. Lantz CA, Nebenzahl E. Behavior and interpretation of the kappa statistic: resolution of the two paradoxes. Journal of clinical epidemiology. 1996;49(4):431-4. 
16. Koeneman MM, Kruse AJ, Kooreman LF, Zur Hausen A, Hopman AH, Sep S], et al. TOPical Imiquimod treatment of high-grade Cervical intraepithelial neoplasia (TOPIC trial): study protocol for a randomized controlled trial. BMC cancer. 2016;16(1):132.

17. Choung RS LIG, Schleck CD, Ziegenfuss JY, Beebe T], Zinsmeister AR, Talley N]. A low response rate does not necessarily indicate non-response bias in gastroenterology survey research: a population-based study. J Public Health. 2013(21):87-95.

18. Kyrgiou M, Mitra A, Arbyn M, Stasinou SM, Martin-Hirsch P, Bennett P, et al. Fertility and early pregnancy outcomes after treatment for cervical intraepithelial neoplasia: systematic review and meta-analysis. Bmj. 2014;349:g6192. 


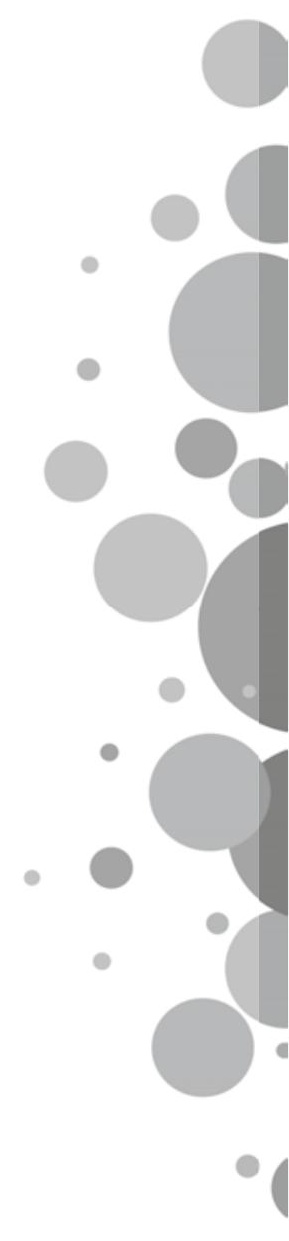




\section{CHAPTER 9a}

\section{TOPical Imiquimod treatment of high-grade Cervical intraepithelial neoplasia (TOPIC trial): study protocol for a randomized controlled trial}

Margot M Koeneman, Arnold] Kruse, Loes FS Kooreman, Axel zur Hausen, Anton HN Hopman, Simone JS Sep, Toon Van Corp, Brigitte FM Slangen, Heleen ] van Beekhuizen, Anna JM van de Sande, Cees G Cerestein, Hans W Nijman, Roy FPM Kruitwagen

BMC Cancer. 2016 Feb 20;16:132. 


\begin{abstract}
Background: Cervical intraepithelial neoplasia $(\mathrm{CIN})$ is the premalignant condition of cervical cancer. Whereas not all high-grade CIN lesions progress to cervical cancer, the natural history and risk of progression of individual lesions remains unpredictable. Therefore, high-grade CIN is currently usually treated by surgical excision: large loop excision of the transformation zone (LLETZ). This procedure has potential complications, such as acute haemorrhage, prolonged bleeding, infection and preterm birth in subsequent pregnancies. These complications could be prevented by development of a non-invasive treatment modality, such as topical imiquimod treatment. The primary study objective is to investigate the efficacy of topical imiquimod $5 \%$ cream for the treatment of high-grade $\mathrm{CIN}$ and to develop a biomarker profile to predict clinical response to imiquimod treatment. Secondary study objectives are to assess treatment side effects, disease recurrence and quality of life during and after different treatment modalities.
\end{abstract}

Methods/Design: The study design is a randomized controlled trial.140 women with a histological diagnosis of high-grade CIN (CIN 2-3) will be randomized into two arms: imiquimod treatment during 16 weeks (experimental arm) or immediate LLETZ (standard care arm). Treatment efficacy will be evaluated by colposcopy with diagnostic biopsies at 20 weeks for the experimental arm. Successful imiquimod treatment is defined as regression to CIN 1 or less, successful LLETZ treatment is defined as PAP 1 after 6 months. Disease recurrence will be evaluated by cytology at 6 , 12 and 24 months after treatment. Side effects will be evaluated using a standardized report form. Quality of life will be evaluated using validated questionnaires at baseline, 20 weeks and one year after treatment. Biomarkers, reflecting both host and viral factors in the pathophysiology of CIN, will be tested at baseline with the aim of developing a predictive biomarker profile for the clinical response to imiquimod treatment.

Discussion: Treatment of high-grade CIN lesions with imquimod in a selected patient population may diminish complications as a result of surgical intervention. More knowledge on treatment efficacy, side effects and long-term recurrence rates after treatment is necessary.

\title{
Trial registration
}

EU Clinical Trials Register EU-CTR2013-001260-34

Medical Ethical Committee approval number: NL44336.068.13 (Medical Ethical Committee Maastricht University Hospital, University of Maastricht)

Affiliation: Maastricht University Hospital

Registration number ClinicalTrials.gov: NCT02329171 


\section{Background}

Cervical intraepithelial neoplasia (CIN) is the premalignant condition of cervical cancer and is caused by cervical human papillomavirus (HPV) infection. [1] The natural history of individual CIN lesions is unpredictable. Approximately $30 \%$ of high-grade CIN progresses to cervical cancer.[2, 3] On the contrary, recent evidence suggests that spontaneous regression occurs in approximately 20-40\% of high-grade lesions. [4-7] Current histopathological assessment is unable to differentiate between lesions that will progress to cervical cancer and those that will regress spontaneously. Therefore, all high-grade CIN lesions are currently treated by surgical excision, consisting of large loop excision of the transformation zone (LLETZ). This treatment is associated with potential complications. Short term complications include pain, vaginal discharge and bleeding. The most serious late complication is premature birth in subsequent pregnancy, probably due to cervical insufficiency.[8-10] Evidence shows a twofold increase in premature birth between 32/34 and 37 weeks in patients who were treated with LLETZ. Furthermore, cervical surgery for CIN may be associated with subfertility. A recent case-control study in 152 patients who underwent cervical surgery showed a twofold increase in prolonged time to conception ( $16.4 \%$ vs $8.6 \%$ ) for patients who underwent LLETZ.[11] Since cervical dysplasia is most common in women of childbearing age, these potential complications are of special interest to the patient population and surgical intervention should be avoided if possible. Therefore, an effective non-invasive treatment modality is needed. A potential agent in non-invasive therapy is imiquimod cream. Imiquimod is an immunomodulator with antiviral and anti-tumour effects. It is a toll-like receptor 7 agonist and induces up regulation of interferon and activation of dendritic cells. [12] It is currently used in treatment of basal cell carcinoma, actinic keratosis and external genital warts in adults. As an offlabel drug, it has also been proven effective in the treatment of HPV related vulvar intraepithelial neoplasia (VIN).[13] The use of imiquimod in CIN has been studied by several authors. [7, 14, 15] Only one randomized controlled trial was conducted, evaluating the efficacy of imiquimod treatment in high-grade CIN.[7] Grimm et al. included 59 patients, who were randomized for treatment with imiquimod or placebo during 16 weeks. The study results are promising: both histologic regression and complete remission of high-grade $\mathrm{CIN}$ was significantly more frequent in patients treated with imiquimod, compared to the control arm ( $73 \%$ vs $39 \%$ and $47 \%$ vs $14 \%$ respectively). However, side effects seem common and long-term outcomes are unknown.

Ideally, the response to imiquimod treatment of an individual patient would be predictable. Previous studies have shown that the natural behaviour of CIN lesions can be partially predicted by biomarker models, consisting of markers that reflect host, viral and cellular factors.[4, 16] This study aims to develop a similar biomarker prediction model for the individual response to imiquimod treatment, in order to enable individualized treatment of CIN lesions.

This study aims to confirm the short and long term efficacy of imiquimod $5 \%$ cream in the treatment of high-grade $\mathrm{CIN}$, as well as to evaluate clinical applicability by assessment of side effects and quality of life during and after treatment. Additionally, it aims to develop a biomarker prediction model for clinical response to imiquimod treatment of high-grade CIN. For this purpose, we designed a randomized controlled trial with two arms, in which imiquimod 
treatment is compared to standard treatment by LLETZ. The trial was designed according to the CONSORT guidelines.

\section{Methods}

\section{Setting and study population}

The study will be started at the outpatient clinic of gynecologic oncology, Maastricht University Medical Center, the Netherlands and is intended as a multi-center trial in the future. Inclusion criteria are newly diagnosed, histologically confirmed high-grade CIN lesions (CIN 2-3) and age above 18 years. Exclusion criteria are previous histologically confirmed high-grade CIN (CIN 2-3), concomitant vulvar and/or vaginal intraepithelial neoplasia, previous cervical malignancy, current malignant disease, immunodeficiency (including HIV/AIDS and immunodepressive medication), pregnancy or lactation and legal incapability.

\section{Study objectives and outcome measures}

The primary study objectives and outcome measures are:

1. Assessment of treatment efficacy of imiquimod treatment for high-grade $\mathrm{CIN}$, as compared to LLETZ treatment. Successful treatment for the experimental (imiquimod) arm is defined as regression to $\mathrm{CIN} 1$ or less in diagnostic biopsies at 20 weeks follow-up. Successful treatment for the LLETZ arm is defined as normal cytology at 6 months follow-up. Based upon earlier studies, we hypothesize that $50-75 \%$ of patients in the experimental (imiquimod) arm will show regression to $\mathrm{CIN} 1$ or less.

These two different outcome measures (cytology in the LLETZ arm and histology in the experimental arm) were selected in order to optimize the assessment of treatment efficacy, whilst limiting overtreatment of patients (performing unnecessary biopsies or LLETZ treatments). For the experimental arm, it is assessed by colposcopy with diagnostic biopsies and for the LLETZ arm it is assessed by cytology. Regarding efficacy of LLETZ treatment, the significance of resection margins of the LLETZ specimen is controversial and is not advised in guidelines as outcome measure of LLETZ efficacy. Therefore, regular follow-up cytology at six months was selected as outcome measure, as is also done in clinical practice and by other authors. Regarding imiquimod treatment, histological assessment of treatment efficacy was selected as outcome measure, in order to optimize the assessment of potential residual disease. Biopsies were chosen rather than a standard LLETZ procedure to evaluate residual disease, to prevent overtreatment.

2. Development of a biomarker model to predict adequate response to imiquimod treatment.

Secondary study objectives and outcome measures are:

1. To determine the incidence and severity of side effects of LLETZ and imiquimod therapy, scored by the Common Terminology Criteria for Adverse Events guidelines.

2. To estimate disease recurrence rates for both arms at 6,12 and 24 months follow-up, defined as abnormal cervical cytology. The follow-up term starts after treatment is finished. 
3. To assess Quality of life (QoL) for both arms before, during and after treatment (at $\mathrm{O}$ and 20 weeks and after 1 year) by the following QoL questionnaires: (1) Medical Outcomes Study 36-Item Short-Form General Health Survey (RAND 36), to assess generic health-related quality of life, (2) the European Organization for Research and Treatment of Cancer (EORTC) QLQ-C30, to assess cancer-specific health-related quality of life, and (3) the European Organization for Research and Treatment of Cancer (EORTC) QLQ-CX24, to assess cervical cancer specific quality of life, including sexual functioning.

\section{Interventions}

After informed consent is obtained, patients are equally randomized into one of two arms:

1. Experimental arm. Patients in this arm are treated by a 16 -week regime of imiquimod $5 \%$ cream.

2. Standard arm. Patients in this arm receive standard treatment by LLETZ.

Follow-up visits are anchored to the start of the treatment (either imiquimod or LLETZ). Cytological assessment will be performed by two independent trained cytology analysts, according to the Papanicolaou system. In case of inconsistent results, a consensus will be reached by discussion. The histopathological assessment of cervical biopsies will be performed by two independent pathologists according to national guidelines, based on the WHO guidelines. In case of inconsistent results, a consensus will be reached by discussion. CIN diagnosis will be based on evaluation of histological features concerned with differentiation, maturation and stratification of cells and nuclear abnormalities, in combination with p16 staining. In CIN 1 there is good maturation with minimal nuclear abnormalities and few mitotic figures. Undifferentiated cells are confined to the deeper layers (lower third) of the epithelium. Mitotic figures are present, but not very numerous. Cytopathic changes due to HPV infection may be observed in the full thickness of the epithelium. CIN 2 is characterized by dysplastic cellular changes mostly restricted to the lower half or the lower two-thirds of the epithelium, with more marked nuclear abnormalities than in CIN 1. Mitotic figures may be seen throughout the lower half of the epithelium. In CIN 3 , differentiation and stratification may be totally absent or present only in the superficial quarter of the epithelium with numerous mitotic figures. Nuclear abnormalities extend throughout the thickness of the epithelium. Many mitotic figures have abnormal forms.

Patients in the experimental arm are treated with imiquimod 5\% cream during 16 weeks. Imiquimod 5\% cream is administered in a vaginal applicator, containing $12,5 \mathrm{mg}$ of imiquimod (one sachet). The cream is administered three times per week. The cream is administered by patients themselves at night, before going to bed. A vaginal shower is performed in the morning in order to remove cream remainders. In case of mild systemic drug-related side effects, patients are offered a prescription for anti-inflammatory drugs (paracetamol or NSAID). In case of more severe or persistent systemic or severe local side effects (Common Terminology Criteria for Adverse Events grade 2 or higher), the frequency of imiquimod application is decreased to twice per week, and subsequently to once per week if side effects persist. Imiquimod treatment is discontinued if side effects are unacceptable to patients hereafter. Subjects should use adequate contraception in order to prevent pregnancy. Subjects should not have vaginal sexual intercourse from the time 
of application of the imiquimod cream until the vaginal shower the next morning. A control colposcopy with diagnostic biopsies is performed after ten weeks to rule out disease progression. Biopsies are performed at the initial high-grade CIN lesion site and at any other suspect site, with a minimum of two. In case of progressive disease (defined as increase in lesions size with stable disease grade, higher disease grade or invasive disease), surgical excision is performed.

Treatment efficacy for the experimental arm is evaluated at 20 weeks follow-up, by colposcopy with diagnostic biopsies. Biopsies are performed by using a cervical biopsy specimen forceps (Aesculap ER055R), at the initial high-grade CIN lesion site and at any other suspect site, with a minimum of two. In case of persistent or progressive disease ( $>\mathrm{CIN} 1)$, surgical excision is performed.

Patients in the standard treatment arm undergo LLETZ at short term (within 4 weeks after the diagnosis). Excision of the transformation zone and macroscopic lesions is performed by a monopolar loop electrode, under local anaesthesia. A summary of study interventions can be found in figure 1.

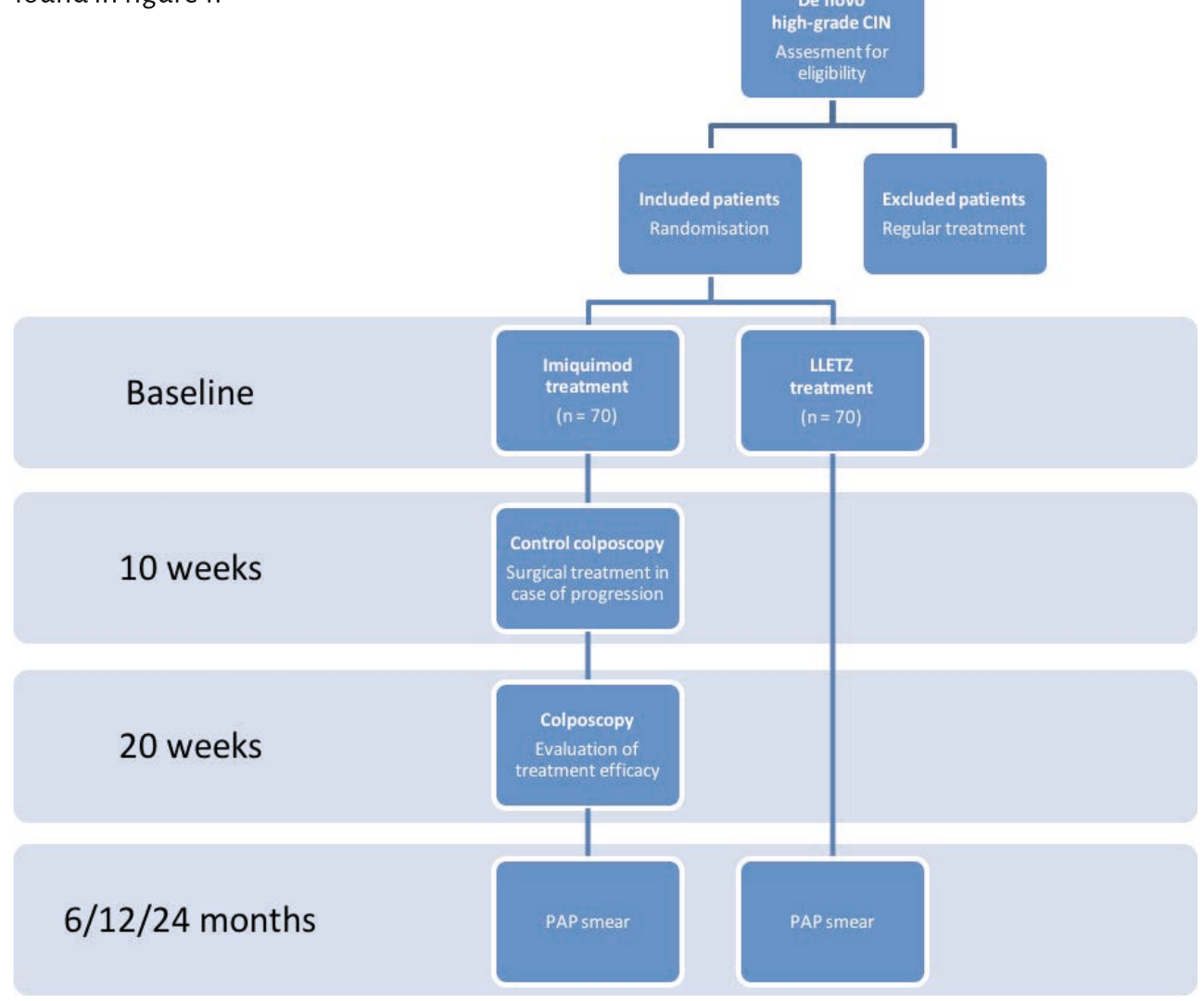

Figure 1. Summary of study interventions 
The imiquimod treatment period was set at 20 weeks, in order to realize adequate treatment efficacy of imiquimod, while minimizing the risk of progression of cervical dysplasia to invasive disease. Progression of CIN into cervical cancer is considered to be a slow process. The annual risk of progression of $\mathrm{CIN} 3$ to invasive cervical cancer is estimated to be less than 1\%.[17] We identified ten studies in which patients with high-grade $\mathrm{CIN}$ underwent watchful waiting for a set period of time. $[4,6,7,18-24]$ A total of 637 patients were included either as a control group, receiving no treatment during 6 weeks to 15 months, or followed during the period between diagnosis and LLETZ. Three cases of invasive disease were identified: all occurred in the same study after 16 weeks of observation. The possibility of invasive disease already present at the initial colposcopy (due to biopsy error) cannot be excluded. Based on these results, we set the maximum treatment period at 20 weeks and we included an additional control colposcopy with diagnostic biopsies after ten weeks. When no disease progression is detected during this colposcopy, the imiquimod treatment can be continued until the 20 weeks colposcopy.

\section{Sample size calculation}

The sample size calculation was based on a regression rate of $73 \%$ at 20 weeks follow-up after immunotherapy and a $95 \%$ treatment efficacy of LLETZ. Using $80 \%$ power and alpha $=5 \%$, the estimated sample size required is 42 women in both arms. Allowing for a withdrawal rate of approximately $20 \%, 53$ women will have to be recruited in each arm. Sample size calculations for the secondary outcome measures indicated that roughly 70 patients per treatment arm would be necessary. Although the sample size calculation should be based on the primary outcome measure, we decided to recruit a total of 140 patients (70 per arm).

\section{Randomization}

Randomization is performed by the principal investigator to prevent selection and allocation bias, by use of a computerized randomization tool. Sampling is stratified according to the following age categories: 18-24, 25-39, and 40 years and older. Sampling will be stratified according to study centre.

\section{Data collection}

Coded data are stored both on paper and in an electronic database. Collected data are stored in a digital case report form (CRF). Raw data is available only to the principal and coordinating investigator. The following data are recorded:

\section{Baseline}

- Patients characteristics: age, ethnical background, education, medical history, smoking, sexual behaviour

- $\quad$ HPV genotyping for all patients

- Histological biomarkers on biopsies of patients in the experimental arm: markers of lymphoproliferative response: CD4, CD8, CD25, CD138, fox p3; cell cycle markers: p16, Rb, p53, Ki67, CK 13, CK 14, IMP3

- Quality of life 
6 weeks follow-up

- $\quad$ Adverse effects of imiquimod treatment: patient reported side effects and side effects noticed at clinical investigation

- $\quad$ Adverse effects of LLETZ treatment: patient reported side effects, using a standardized report form

10 weeks follow-up

- Treatment compliance: amount of applied doses of imiquimod, as documented by the study subject on a dose calendar

- Histological presence and grading of CIN for the experimental arm

- $\quad$ Adverse effects of imiquimod treatment: patients reported side effects and side effects noticed at clinical investigation

14 weeks follow-up

- $\quad$ Adverse effects of imiquimod treatment: patients reported side effects and side effects noticed at clinical investigation

20 weeks follow-up

- Treatment compliance: amount of applied doses of imiquimod, as documented by the study subject on a dose calendar

- Histological presence and grading of CIN for the experimental arm

- $\quad$ Adverse effects of imiquimod treatment: patients reported side effects and side effects noticed at clinical investigation

- Quality of life

6,12, 24 months follow-up

- Cervical cytology outcomes for all patients, including HPV genotyping

- Quality of life for all patients at 12 months follow-up

\section{Statistical methods}

Logistic regression analysis will be used to evaluate treatment efficacy of imiquimod treatment, compared to LLETZ treatment. Covariates in this analysis are age at diagnosis, CIN grade, smoking and HPV-subtype. Analysis will be based on intention-to-treat protocol. Biomarker prediction models for adequate response to imiquimod treatment will be developed by backward logistic regression analysis of biomarkers based on likelihood ration tests. The prevalence and severity of side effects of imiquimod and LLETZ treatment will be presented as proportions and means with $95 \%$ confidence intervals. Differences in the rates of overall side effects and severe side effects between the imiquimod and LLETZ arms will be tested with a chi-square test. Disease recurrence rates after 6,12 and 24 months will be evaluated in successfully treated patients by use of multiple logistic regression analysis, after adjustment for age at diagnosis, $\mathrm{CIN}$ grade, smoking sexual behaviour and HPV subtype. Repeated-measures analysis of variance will be used to test for between-group differences over time in Quality of Life scores. 
Analysis of covariance will be used to compare group scores on these outcomes at 20 weeks and at 12 months, with adjustment for baseline scores.

\section{Withdrawal of individual subjects and replacement}

Subjects can leave the study at any time for any reason, without consequences. The investigator can decide to withdraw a subject from the study for medical reasons. Withdrawn individuals are not replaced by a new volunteer. Patients who withdraw from the study are offered the standard treatment of CIN, being a LLETZ procedure with standard follow up.

\section{Ethical considerations and dissemination}

The study was approved of by the Medical Ethical Committee of Maastricht University Hospital, University of Maastricht. The study will be performed according to the standards outlined in the Declaration of Helsinki. Ethics committee approval has been completed. Monitoring of the study is performed by a Data and Safety Monitoring Board, appointed by the Clinical Trial Center Maastricht. Adverse events are recorded and reported according to local protocol. Study results will be offered for publication in an international medical journal. Study results will be communicated to trial participants by mail, if agreed upon by the participant.

\section{Substantial amendment}

The current study protocol includes a substantial amendment to the original study protocol, which consisted of three study arms: imiquimod treatment arm, LLETZ treatment arm and an observational arm. The purpose of the observational arm was to assess spontaneous regression of high-grade $\mathrm{CIN}$ and to develop a prognostic biomarker panel to predict spontaneous regression of high-grade CIN. Patients in the observational arm underwent no treatment for a period of maximum 20 weeks. Histological assessment of disease development was performed after 10 and 20 weeks by colposcopy with diagnostic biopsies. Inclusion of patients into the study was hampered by the observational arm: patients declined the study because they wished to be treated, rather than undergo observational management. The observational arm was removed from the study.

\section{Discussion}

The development of a non-invasive treatment modality for high-grade CIN lesions may diminish complications as a result of surgical intervention. An earlier study indicates that imiquimod induces disease regression in $73 \%$. [7, 13] Thus, imiquimod treatment may prevent surgical treatment in the majority of patients. The current study aims to test the treatment efficacy hypothesis as well as long term disease recurrence after treatment and clinical applicability of imiquimod treatment, defined as side effects and quality of life during and after treatment. Furthermore, it aims to develop a prediction model for clinical response to imiquimod treatment, based on histological biomarkers. 


\section{Trial status}

Approved by the Medical Ethical Committee Maastricht University Hospital, University of Maastricht, on 21 May 2014. Recruitment started in December 2014. Protocol version 5.0. 


\section{References}

1. zur Hausen H. Papillomaviruses causing cancer: evasion from host-cell control in early events in carcinogenesis. Journal of the National Cancer Institute. 2000;92(9):690-8.

2. McCredie MR, Sharples K], Paul C, Baranyai ], Medley G, Jones RW, et al. Natural history of cervical neoplasia and risk of invasive cancer in women with cervical intraepithelial neoplasia 3: a retrospective cohort study. The lancet oncology. 2008;9(5):425-34.

3. Peto ], Gilham C, Deacon J, Taylor C, Evans C, Binns W, et al. Cervical HPV infection and neoplasia in a large population-based prospective study: the Manchester cohort. British journal of cancer. 2004;91(5):942-53.

4. Munk AC, Gudlaugsson E, Ovestad IT, Lovslett K, Fiane B, Hidle B, et al. Interaction of epithelial biomarkers, local immune response and condom use in cervical intraepithelial neoplasia 2-3 regression. Gynecologic oncology. 2012;127(3):489-94.

5. Ovestad IT, Gudlaugsson E, Skaland I, Malpica A, Munk AC, Janssen EA, et al. The impact of epithelial biomarkers, local immune response and human papillomavirus genotype in the regression of cervical intraepithelial neoplasia grades 2-3. J Clin Pathol. 2011;64(4):303-7.

6. Trimble CL, Piantadosi S, Gravitt P, Ronnett B, Pizer E, Elko A, et al. Spontaneous regression of high-grade cervical dysplasia: effects of human papillomavirus type and HLA phenotype. Clin Cancer Res. 2005;11(13):4717-23.

7. Grimm C, Polterauer S, Natter C, Rahhal ], Hefler L, Tempfer CB, et al. Treatment of cervical intraepithelial neoplasia with topical imiquimod: a randomized controlled trial. Obstetrics and gynecology. 2012;120(1):152-9.

8. Crane JM. Pregnancy outcome after loop electrosurgical excision procedure: a systematic review. Obstetrics and gynecology. 2003;102(5 Pt 1):1058-62.

9. Arbyn M, Kyrgiou M, Simoens C, Raifu AO, Koliopoulos G, Martin-Hirsch P, et al. Perinatal mortality and other severe adverse pregnancy outcomes associated with treatment of cervical intraepithelial neoplasia: meta-analysis. Bmj. 2008;337:a1284.

10. Kyrgiou M, Koliopoulos G, Martin-Hirsch P, Arbyn M, Prendiville W, Paraskevaidis E. Obstetric outcomes after conservative treatment for intraepithelial or early invasive cervical lesions: systematic review and meta-analysis. Lancet. 2006;367(9509):489-98.

11. Spracklen CN, Harland KK, Stegmann B], Saftlas AF. Cervical surgery forcervical intraepithelial neoplasia and prolonged time to conception of a live birth: a case-control study. BJOC : an international journal of obstetrics and gynaecology. 2013;120(8):960-5.

12. Stary G, Bangert C, Tauber M, Strohal R, Kopp T, Stingl C. Tumoricidal activity of TLR7/8activated inflammatory dendritic cells. J Exp Med. 2007;204(6):1441-51.

13. van Seters $M$, van Beurden $M$, ten Kate F], Beckmann I, Ewing PC, Eijkemans M], et al. Treatment of vulvar intraepithelial neoplasia with topical imiquimod. N Engl ] Med. 2008;358(14):1465-73.

14. Pachman DR, Barton DL, Clayton AC, McGovern RM, Jefferies JA, Novotny PJ, et al. Randomized clinical trial of imiquimod: an adjunct to treating cervical dysplasia. American journal of obstetrics and gynecology. 2012;206(1):42 e1-7.

15. Lin CT, Qiu JT, Wang C], Chang SD, Tang YH, Wu P], et al. Topical imiquimod treatment for human papillomavirus infection in patients with and without cervical/vaginal intraepithelial 
neoplasia. Taiwanese journal of obstetrics \& gynecology. 2012;51(4):533-8.

16. Baak JP, Kruse AJ, Robboy S], Janssen EA, van Diermen B, Skaland I. Dynamic behavioural interpretation of cervical intraepithelial neoplasia with molecular biomarkers. ] Clin Pathol. 2006;59(10):1017-28.

17. Canfell K, Barnabas R, Patnick ], Beral V. The predicted effect of changes in cervical screening practice in the UK: results from a modelling study. British journal of cancer. 2004;91(3):530-6.

18. Follen M, Atkinson EN, Schottenfeld D, Malpica A, West L, Lippman S, et al. A randomized clinical trial of 4-hydroxyphenylretinamide for high-grade squamous intraepithelial lesions of the cervix. Clin Cancer Res. 2001;7(11):3356-65.

19. Meyskens FL, Jr., Surwit E, Moon TE, Childers JM, Davis JR, Dorr RT, et al. Enhancement of regression of cervical intraepithelial neoplasia II (moderate dysplasia) with topically applied all-trans-retinoic acid: a randomized trial. Journal of the National Cancer Institute. 1994;86(7):539-43.

20. Keefe KA, Schell M], Brewer C, McHale M, Brewster W, Chapman JA, et al. A randomized, double blind, Phase III trial using oral beta-carotene supplementation for women with highgrade cervical intraepithelial neoplasia. Cancer epidemiology, biomarkers \& prevention : a publication of the American Association for Cancer Research, cosponsored by the American Society of Preventive Oncology. 2001;10(10):1029-35.

21. Alvarez RD, Conner MG, Weiss H, Klug PM, Niwas S, Manne U, et al. The efficacy of 9-cisretinoic acid (aliretinoin) as a chemopreventive agent for cervical dysplasia: results of a randomized double-blind clinical trial. Cancer epidemiology, biomarkers \& prevention : a publication of the American Association for Cancer Research, cosponsored by the American Society of Preventive Oncology. 2003;12(2):114-9.

22. Garcia F, Petry KU, Muderspach L, Gold MA, Braly P, Crum CP, et al. ZYC101a for treatment of high-grade cervical intraepithelial neoplasia: a randomized controlled trial. Obstetrics and gynecology. 2004;103(2):317-26.

23. Van Pachterbeke C, Bucella D, Rozenberg S, Manigart Y, Gilles C, Larsimont D, et al. Topical treatment of $\mathrm{CIN} 2+$ by cidofovir: results of a phase II, double-blind, prospective, placebocontrolled study. Gynecologic oncology. 2009;115(1):69-74.

24. Kaufmann AM, Nieland JD, Jochmus I, Baur S, Friese K, Gabelsberger ], et al. Vaccination trial with HPV16 L1E7 chimeric virus-like particles in women suffering from high grade cervical intraepithelial neoplasia (CIN 2/3). International journal of cancer Journal international du cancer. 2007;121(12):2794-800. 



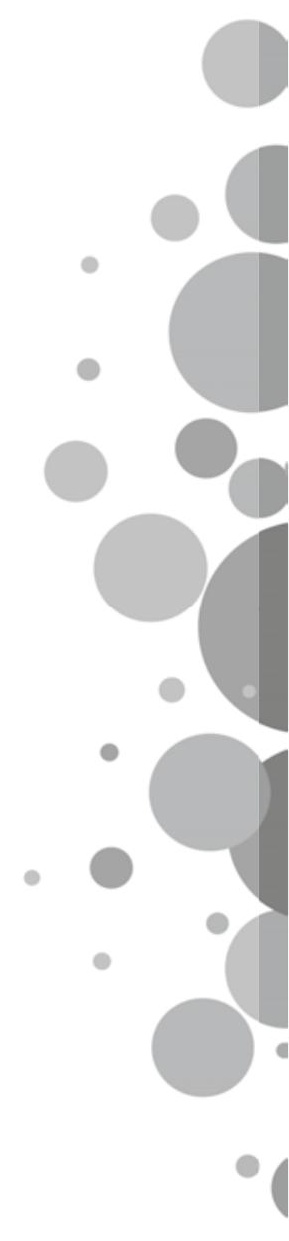




\title{
CHAPTER 9b
}

\author{
Preliminary stop
}

of the TOPical Imiquimod treatment of high-grade Cervical intraepithelial neoplasia (TOPIC) trial.

Margot M Koeneman, Arnold] Kruse, Loes FS Kooreman, Axel zur Hausen, Anton HN Hopman, Simone JS Sep, Toon Van Corp, Brigitte FM Slangen, Heleen ] van Beekhuizen, Anna JM van de Sande, Cees C Cerestein, Hans W Nijman, Roy FPM Kruitwagen

BMC Cancer. 2017 Feb 7;17(1):110. 


\section{Abstract}

The "TOPical Imiquimod treatment of high-grade Cervical intraepithelial neoplasia" (TOPIC) trial was stopped preliminary, due to lagging inclusions. This study aimed to evaluate the treatment efficacy and clinical applicability of imiquimod 5\% cream in high-grade cervical intraepithelial neoplasia (CIN). The lagging inclusions were mainly due to a strong patient preference for either of the two treatment modalities. This prompted us to initiate a new study on the same subject, with a non-randomized, open-label design: the 'TOPical Imiquimod treatment of high-grade Cervical intraepithelial neoplasia (TOPIC)-3' study. 


\section{Correspondence}

The TOPical Imiquimod treatment of high-grade Cervical intraepithelial neoplasia (TOPIC) trial started in January 2015, with the aim to evaluate the treatment efficacy and clinical applicability of imiquimod $5 \%$ cream in high-grade cervical intraepithelial neoplasia (CIN). A study protocol for this study was published in BMC Cancer in February 2016.[1] Patients were randomized into one of two treatment arms: the imiquimod treatment arm, in which subjects were treated with imiquimod during 16 weeks, and the standard treatment arm, in which large loop excision of the transformation zone (LLETZ) was performed. An earlier version of the study included a third treatment arm: an observational arm, which consisted of 'watchful waiting' during 20 weeks, with the aim to evaluate spontaneous regression of high-grade $\mathrm{CIN}$ and identify prognostic biomarkers for spontaneous regression. The observational arm was removed from the study after 9 months to increase the inclusion rate. The current study stopped preliminary in May 2016 due to lagging inclusions.

The lagging inclusions may be explained by the very different nature of the treatment modalities and the strong preferences of women for either imiquimod or LLETZ treatment. We experienced that women have a general preference for LLETZ treatment, as it provides a fast and effective treatment of high-grade CIN. This was confirmed by a recent patient preference study performed by the author (data not published). This preference study also showed that a preference for imiquimod treatment is largely restricted to women with a future pregnancy wish and that a treatment efficacy of at least $72 \%$ is desired by these women. Indeed, we experienced that only a subset of women with a future pregnancy wish wanted to participate in the study. All of these women had a strong preference for imiquimod treatment. The amount of women willing to participate was too small to achieve the intended study population of 120 in one study centre, within the time-frame of the study. For this reason, the study was preliminary stopped.

Twelve women were included in the total duration of the study: six women were included in the observational arm, three in the imiquimod arm and three in the standard treatment arm. Of the women included in the observational arm, two showed spontaneous regression to $\mathrm{CIN} 1$ or less. Two women showed persistent $\mathrm{CIN} 2$ after 10 and 20 weeks and were subsequently treated by LLETZ. The other two women quit the study immediately after randomization and were treated by LLETZ. Of the three women who were randomized for imiquimod treatment, one quit the study immediately after randomization and was treated by LLETZ. The other two women completed imiquimod treatment and both showed disease regression to $\mathrm{CIN} 1$ or less.

As a consequence of the very different nature of the two treatment modalities and the differences in treatment preferences among different populations of women, imiquimod will most likely not develop as a treatment alternative for all women with high-grade $\mathrm{CIN}$, but may be restricted to women with recurrent lesions or a future pregnancy wish. These insights prompted us to initiate a new study concerning the treatment efficacy and clinical applicability of imiquimod $5 \%$ cream in high-grade $\mathrm{CIN}$, in selected populations of women with a preference for either of the two treatment modalities. The 'TOPical Imiquimod treatment of high-grade Cervical intraepithelial 


\section{Chaptergb}

neoplasia (TOPIC)-3 study' is a multicenter, open-label, non-randomized, controlled study, evaluating treatment efficacy, side effects and quality of life associated with imiquimod treatment of high-grade CIN lesions in a selected population of women who prefer imiquimod treatment instead of LLETZ. The study also aims to develop a biomarker profile to predict clinical response to imiquimod treatment. This enables selection of patients in which good treatment response is expected. The study was approved by the Medical Ethics Committee AZM/UM (approval number METC162025) and has been registered on ClinicalTrials.gov (ClinicalTrials.gov Identifier:NCT02917746, 16 September 2016). The study started recruiting patients in November 2016. 


\section{References}

1. Koeneman MM, Kruse A], Kooreman LF, Zur Hausen A, Hopman AH, Sep S], Van Corp T, Slangen BF, van Beekhuizen H], van de Sande M, Cerestein CG, Nijman HW, Kruitwagen RF. TOPical Imiquimod treatment of high-grade Cervical intraepithelial neoplasia (TOPIC trial): study protocol for a randomized controlled trial. BMC Cancer. 2016 Feb 20;16:132 


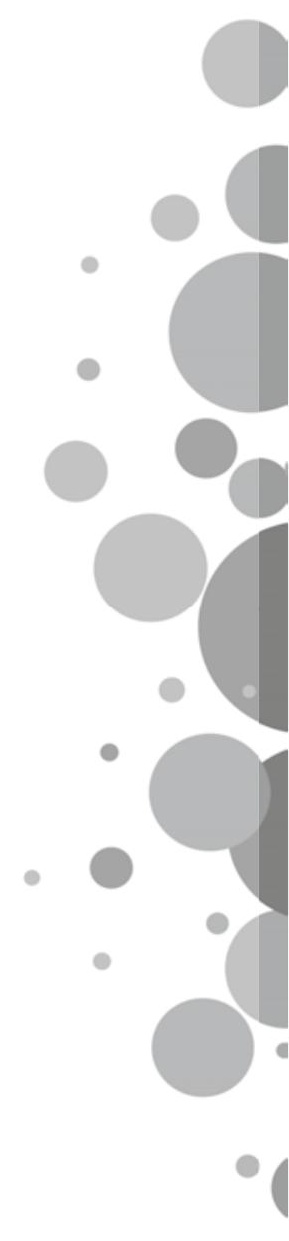




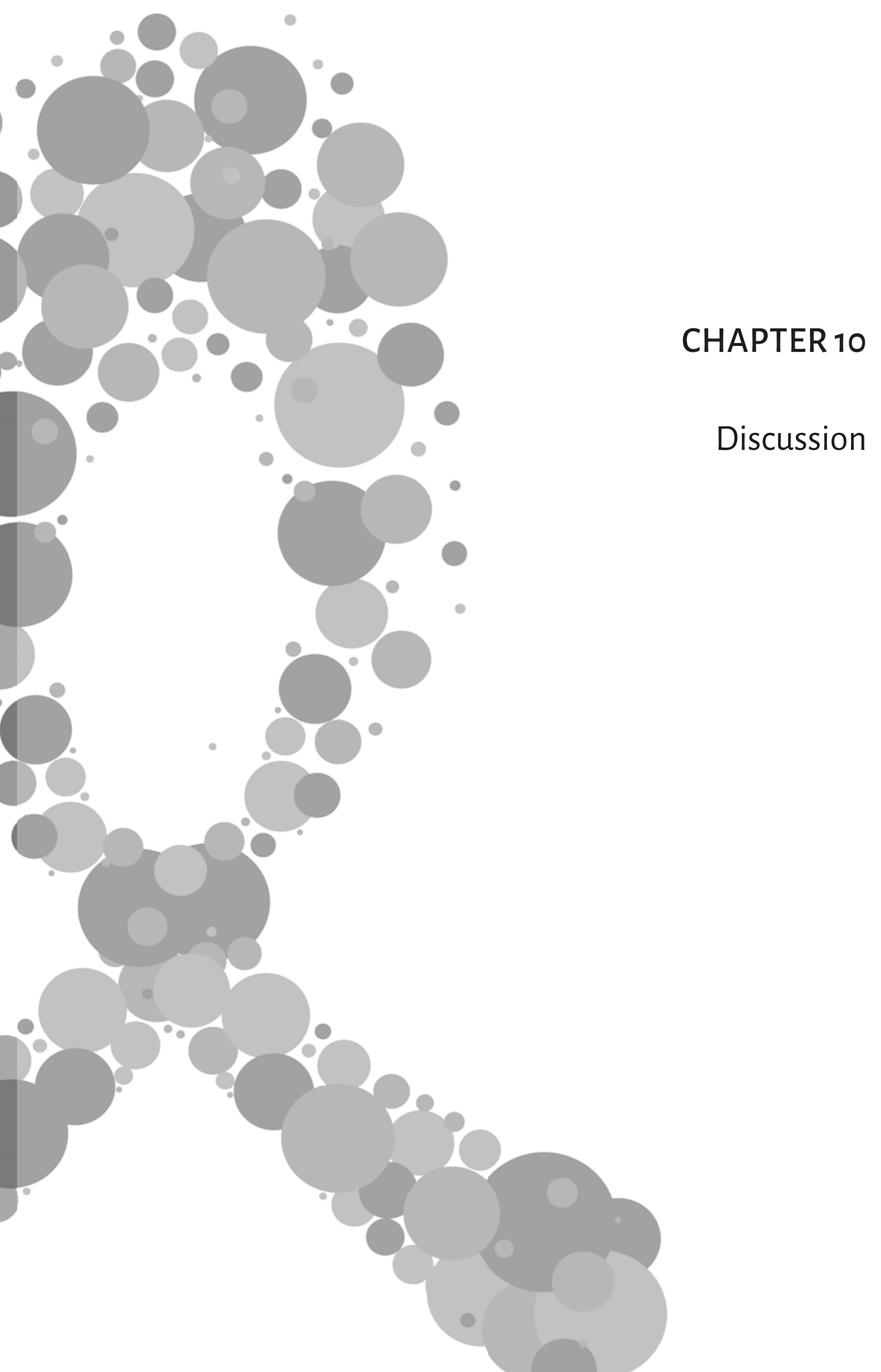



Treatment of high-grade CIN lesions is an effective method to prevent the development of cervical carcinoma. However, since not all lesions will progress to cervical cancer, overtreatment of highgrade CIN is common.[1] This leads to unnecessary side effects of surgical treatment, of which an increased risk of premature birth in subsequent pregnancy is the most severe.[2] With 5,000 women being treated for high-grade $\mathrm{CIN}$ yearly in the Netherlands, this is a relevant problem. The current HPV vaccination program will not eradicate all high-grade CIN lesions. At the same time, recent implementation of HPV-based cervical cancer screening leads to an increase in diagnosed high-grade $\mathrm{CIN}$ lesions.[3] Consequently, a substantial number of women will remain exposed to potential side effects of surgical treatment of high-grade CIN. Moreover, approximately $15 \%$ of high-grade $\mathrm{CIN}$ lesions are recurrent, leading to repeated surgical intervention, further increasing the risk of premature birth in future pregnancies.[4] Non-surgical treatment modalities could improve management of high-grade $\mathrm{CIN}$, with the aim to reduce side effects of surgical treatment. This thesis provides evidence and tools for such alternative management strategies in high-grade $\mathrm{CIN}$, focusing on the prediction of spontaneous regression with prognostic biomarkers and immunotherapy with imiquimod.

\section{Prognostic biomarkers for spontaneous regression of high-grade CIN}

Biomarker research in cancer is a hot topic and extensive research is being conducted in various fields of medicine, including in CIN and cervical carcinoma. Useful diagnostic markers have been identified and implemented to aid cytological triage and histological diagnosis of high-grade $\mathrm{CIN}$. Among these are HPV-genotype for cytological triage and p16/Ki67 staining for histological diagnosis.[5, 6] Various other cytological diagnostic markers have been studied, some with promising results such as combined CADM1/MAL methylation testing, but none have reached the stage of clinical implementation. [7, 8] Research on prognostic biomarkers in high-grade CIN has also proven challenging, as is shown in this thesis. We reviewed previously studied prognostic biomarkers in high-grade $\mathrm{CIN}$ and assessed their prognostic value and clinical applicability, according to their role in the natural history of CIN and the PROBE criteria for biomarker research. We concluded that none of the reviewed biomarkers is currently eligible for implementation in clinical practice for prediction of disease outcome in high-grade $\mathrm{CIN}$.

Prognostic biomarker research in high-grade CIN faces several important challenges. One of these challenges is the inability to conduct long-term prospective observational studies, due to the risk of malignant evolution of high-grade CIN lesions. This is most clearly illustrated by a historical and unethical clinical study at the National Women's Hospital, Auckland, New Zealand, in which treatment of $\mathrm{CIN}_{3}$ was withheld from a substantial number of women between 1965 and 1974.[9] The cumulative incidence of invasive cancer of the cervix or vaginal vault was $31 \%$ in these women. Consequently, in order to prevent the development of cervical carcinoma, a certain maximum follow-up term should be determined for prognostic biomarker studies in CIN3. A natural history model based on cervical screening practice in the UK showed that the annual risk of progression of a CIN 3 lesion to invasive cervical cancer is 0.99\%.[10] The short term natural history of highgrade $\mathrm{CIN}$ can be further derived from randomized controlled therapeutic trials, which include a control group of histologically confirmed CIN 2/3 patients, and from short-term follow-up studies. 
[11-21] Eleven such studies were identified and included a total of 678 women with CIN2/3 lesions. Follow-up terms ranged from six weeks to two years, during which colposcopies were performed every two to six months. Three cases of progression to micro-invasive disease were described and one case of invasive disease. $[13,15]$ All three cases of micro-invasive disease occurred in a study with a follow-up term of 20 weeks, in which a control colposcopy with biopsies was performed at 8 weeks. The author states that presence of micro-invasive disease at baseline cannot be excluded in these cases. [15] The only case of invasive disease occurred in an RCT with a two year follow-up term, in which control colposcopies were performed every 3 months and biopsies every six months. Overall, four cases of (micro-)invasive disease were diagnosed in 678 women (0.6\%), of which one occurred in a study with a relatively long follow-up term. Based on these study results, short-term prospective observational studies in high-grade $\mathrm{CIN}$ do seem feasible, whilst histological followup is provided at limited intervals. This strategy provides an opportunity for prognostic biomarker research. Short term follow-up, however, does imply that those lesions that would regress after the follow-up limit, will be classified as non-regressing lesions. Indeed, increasing regression rates with longer follow-up have been reported. Munk et al. reported that regression of $\mathrm{CIN} 2 / 3$ was $5 \%$ in women who were treated within 8 weeks, compared to $38 \%$ in women who were treated after 8 weeks follow-up. [22] Codfrey et al. followed 100 women with a CIN2 lesion, of whom 62 women showed spontaneous regression. The time to cytological normalization in these women varied from 3 to 43 months, indicating that spontaneous regression can occur even after longer periods. [23] The inability to conduct long-term studies with associated underestimation of spontaneous regression seems to be an unavoidable limitation to prognostic biomarker research in high-grade $\mathrm{CIN}$. Despite the feasibility of short-term biomarker research, motivating women with a $\mathrm{CIN} 3$ lesion for participation in a natural history study proved to be difficult, as we experienced in the first version of the TOPIC trial. This version included an observational arm, with the aim to develop a prognostic biomarker panel to predict spontaneous regression of high-grade CIN. Patients in the observational arm underwent no treatment for a period of maximum 20 weeks. Histological assessment of disease development was performed after 10 and 20 weeks by colposcopy with diagnostic biopsies. Inclusion of patients into the study was hampered by this observational arm: patients declined the study because they wished to be treated, rather than undergo observational management. The observational arm was removed from the study.

We studied Human Leucocyte Antigen (HLA) types and gain of the human telomerase RNA gene (hTERC) as potential prognostic biomarkers in high-grade CIN. Both were identified in our review as promising candidate markers. We found no effect of the HLA repertoire on spontaneous regression of high-grade $\mathrm{CIN}$ and could therefore not identify HLA alleles as a prognostic biomarker in high-grade CIN. The prognostic properties of hTERC gain were studied by means of 3926 locus, which contains the hTERC gene. A pilot study in 19 women showed that 3926 gain had a high negative predictive value: all lesions without 3926 gain showed disease regression. As such, the absence of 3926 gain could potentially serve as a prognostic biomarker for the identification of CIN lesions with a high probability of disease regression. Nevertheless, the positive predictive value of 3926 gain was limited, leading to incorrect classification as non-regressing lesions in women whose lesion would actually regress. This would mean that a significant subset of women would still be overtreated. 
As such, clinical application of 3926 gain, as a single marker, is limited by suboptimal test characteristics.

The results of our biomarker studies emphasize another important challenge in prognostic biomarker research in high-grade $\mathrm{CIN}$ : the achievement of adequate prognostic test properties. Desirable performance levels depend on the clinical context of the biomarker.[24] In case of highgrade $\mathrm{CIN}$ prognosis, it is vital to identify those lesions that will not regress spontaneously, as these women require treatment to avoid malignant evolution. Consequently, a high specificity of a test in prediction of disease regression is warranted. At the same time, a high sensitivity limits overtreatment, by correct identification of those lesions that will regress spontaneously. In our review, we concluded that no individual biomarker showed sufficient prognostic value to be implemented as a single prognostic test. This may be explained by the fact that individual biomarkers reflect contributing, but not single critical steps, in cervical oncogenesis.

The development of CIN and cervical cancer is characterized by a complex interaction between virus and host, in which viral oncogenic properties and the human immune system influence the cellular processes that lead to cervical oncogenesis. $[25,26]$ In this process, several important molecular events have been identified, among which are for example inactivation of pRB and p53, viral DNA integration and upregulation of telomerase.[25] However, no individual event has been identified as 'point of no return' in disease progression. Indeed, viral DNA integration and upregulation of telomerase is not found in all high-grade CIN lesions or cervical carcinomas and lesions with low pRB/p53 can still regress.[27-29] As such, it is unlikely that the prediction of the natural prognosis of CIN lesions will be based on a marker reflecting one molecular event, but rather on a combination of viral, host and genetic parameters. Therefore, in order to achieve better test performances, we suggest the development of biomarker profiles or prediction models, instead of aiming for individual prognostic markers. Previous research has shown that biomarker combinations improve test performance, when an individual marker does not have sufficient classification accuracy on its own.[30] One such biomarker profile in high-grade CIN has previously been described. [11] This profile was developed in 162 women with high-grade CIN and consists of four markers: the percentage of $\mathrm{pRb}$ in the lower epithelium, lesion size, the amount of $\mathrm{CD}_{4}+$ cells in the stroma and consistent condom use. All four markers are independently associated with disease regression. The model categorizes women into a group with a low regression probability of $8 \%$ and a high regression probability group of $56 \%$. The model was characterized by a sensitivity of $71 \%$ and a specificity of $85 \%$ : 24 out of 34 regressive lesions and $109 / 128$ non-regressive lesions were correctly predicted. This implies that overtreatment would occur in 30\% (10/34) of women and undertreatment in 15\% of women (19/128). As such, the model seems insufficient for implementation in clinical practice, but serves as an example for future research. Biomarker profiles composed of other markers may provide better test characteristics.

\section{Prognostic markers for spontaneous regression of $\mathrm{CIN}$, as a subset of high-grade CIN}

An important consideration regarding observational studies in high-grade $\mathrm{CIN}$ is the distinction between $\mathrm{CIN} 2$ and $\mathrm{CIN}_{3}$ lesions, because spontaneous regression of $\mathrm{CIN} 2$ seems much more common than $\mathrm{CIN} 3$. Natural history studies, in which $\mathrm{CIN} 2$ and $\mathrm{CIN} 3$ lesions were distinguished 
based on conventional pathological criteria, show spontaneous regression rates for $\mathrm{CIN} 2$ of 40 $74 \%$, compared to $22-33 \%$ for $\mathrm{CIN}_{3}$ lesions. $[1,20]$ Other studies confirm the high regression rate of $\mathrm{CIN} 2$ lesions. [31] Based on these results, guidelines now advice upon observational management of $\mathrm{CIN}_{2}$ in younger women, to reduce overtreatment and associated long-term obstetric complications.[32] In clinical histopathological assessment, however, the distinction between $\mathrm{CIN} 2$ and $\mathrm{CIN} 3$ lesions is challenging. Studies show that $\mathrm{CIN}_{2}$ is a much less reproducible diagnosis than $\mathrm{CIN}_{3}$ and distinction between $\mathrm{CIN}_{2}$ and $\mathrm{CIN}_{3}$ is not always clear.[33-36] Concordance in $\mathrm{CIN} 2$ diagnosis between different pathologists was found in only $19-53 \%$ of cases, compared to $48-62 \%$ in $\mathrm{CIN} 3$ lesions. The inconsistency in $\mathrm{CIN} 2$ diagnosis raises discussion as to whether $\mathrm{CIN} 2$ actually corresponds to a well-defined phase of the pathogenetic pathway of infection and transformation in $\mathrm{CIN}$. If $\mathrm{CIN} 2$ is considered not to be individual entity, the histological image of $\mathrm{CIN} 2$ may represent the late stage of a productive infection or the early stage of a transforming infection, which are considered two biologically different phases in the pathogenetic pathway of infection and transformation.[37] Although the histological image of these two forms of $\mathrm{CIN}_{2}$ may look similar, their biological behavior is markedly different. The high regression rate of $\mathrm{CIN} 2$ may then be explained by the fact that a substantial subset of CIN2 lesions represents a productive infection rather than a transforming infection. A solution to this discussion would be a strategy in which the lesion is not classified according to CIN grade, but according to its potential for spontaneous regression. After all, that is the actual clinical relevance of the diagnosis. This brings us back to the importance of prognostic biomarkers in high-grade $\mathrm{CIN}$. While prognostic biomarkers are lacking, other methods should be applied to distinguish between $\mathrm{CIN} 2$ and $\mathrm{CIN} 3$. Uleberg et al. studied 114 water-soluble proteins in supernatants from 20 fresh cervical biopsies (10 CIN2 and $10 \mathrm{CIN} 3$ lesions).[38] The histological diagnosis was based on p16 and Ki67 staining, but no information was provided on HPV status. Cytokeratin (CK) 2 was found to be a strong discriminator between $\mathrm{CIN}_{2}$ and $\mathrm{CIN}_{3}$, with $90 \%$ overall correct classifications. $\mathrm{CK}_{2}$ expression was expressed in the low and middle part of the epithelium in CIN2 lesions and was not expressed in $\mathrm{CIN}_{3}$ lesions. A difference in $\mathrm{CK}_{2}$ expression seems biologically plausible. This cytokeratin is normally expressed in late differentiation of the epidermal layers of normal skin. Its varied expression in CIN indicates that epithelial cells in CIN2 have a greater tendency towards differentiation. [38] These diagnostic properties of CK2 have not been validated in larger studies and have therefore not been implemented in clinical practice. Until the development of better methods, we advocate the distinction of $\mathrm{CIN}_{2}$ and $\mathrm{CIN}_{3}$ lesions based on conventional histopathological criteria, including p16 and Ki67 staining, with the aim to provide young women an opportunity for conservative management in case of $\mathrm{CIN} 2$.

In the era of personalized medicine and shared decision-making, individual prediction of disease outcome in $\mathrm{CIN}_{2}$ would enhance patient counseling with regard to management options. We therefore developed a prediction model for spontaneous regression of CIN2. Since it consists of simple and inexpensive clinical and pathological parameters, it is widely applicable. The model, based on smoking status, PAP smear outcome, concomitant $\mathrm{CIN} 1$ and number of biopsies containing $\mathrm{CIN} 2$, has a reasonable discriminative capacity and is accurate, especially in the prediction of disease regression. Upon external validation, the model could function as a tool for a more individualized approach to $\mathrm{CIN} 2$ management, in a population with mixed hrHPV positive 
and negative lesions. In order to provide with prognostic factors for women with exclusively hrHPV positive CIN2, we performed a second study in this subgroup of women and identified smoking and parity as prognostic factors. Disease regression occurred in $85 \%$ of nulliparous women who did not smoke, as compared to $33 \%$ of smoking, parous women. These findings are in accordance with existing literature on risk factors for high-grade CIN. Smoking is an established risk factor for the development of CIN and cervical carcinoma.[39] Smoking has also previously been associated with disease outcome of $\mathrm{CIN} 2$, in mixed populations of hrHPV positive and negative lesions.[40, 41] Several biological mechanisms for this effect have been proposed, among which are an altered immune response and direct cellular effects of exposure to nicotine and other tobacco products. [39] The immune response in smokers is characterized by an unbalanced production of pro-and anti-inflammatory cytokines, suppression of $\mathrm{T}$ lymphocyte activity and lower numbers of Th cells and NK cells. Moreover, smoking women have decreased numbers of cervical Langerhans cells. An impaired immune response as a result of smoking may lead to less effective clearance of CIN lesions. Direct cellular effects of exposure to nicotine include increased cell proliferation, inhibition of apoptosis and stimulation of vascular endothelial growth factor, all of which contribute to oncogenesis and may reduce the potential of lesion regression. $[39,42,43]$ Parity, too, has been previously associated with an increased risk of CIN and cervical cancer and increased parity has been shown to decrease the likelihood of HPV clearance.[44-46] Interestingly, a recent study by Jensen et al. shows that parity is associated with an increased risk for $\mathrm{CIN} 3$ and cervical cancer in women with a persistent HPV infection. This implies that the association between parity and CIN may not be caused by an increased risk for HPV infection or persistence of HPV infection, but that giving birth itself seems to be a risk factor for high-grade CIN. [47] Proposed mechanisms for this increased risk are increased hormone levels and impaired immune response during pregnancy, increased exposure of the transformation zone in parous women as a result of a longer ectocervical position and local tissue damage during vaginal delivery. In clinical practice, smoking status and parity can be considered in individual patient counseling regarding the choice between immediate treatment or conservative management of hrHPV positive $\mathrm{CIN} 2$ lesions.

\section{Non-surgical treatment of high-grade CIN}

For those women in whom treatment cannot be avoided, because their lesion is a $\mathrm{CIN}_{3}$ or observational management of a CIN2 lesion is unwanted, the development of a non-surgical treatment modality may reduce side effects of surgery. Imiquimod cream is the only non-surgical treatment modality for high-grade CIN incorporated in the Dutch guideline for CIN. Imiquimod is a Toll-like receptor (TLR) agonist, with anti-tumor and anti-viral properties.

\section{Toll like receptors and cervical carcinogenesis}

TLRs are the key molecules of the innate immunity. They are pattern recognition receptors (PRRs) that recognize specific pathogen associated molecular patterns (PAMPs) in viruses and other pathogens.[48-50] Ten functional TLRs have been described in humans, which recognize different PAMPs and are differentially expressed in human tissues. Upon recognition of a PAMP, TLRs trigger the nuclear factor kappa B (NF-kB) signaling pathways to develop antigen-specific acquired immunity, predominantly through pro-inflammatory cytokines interferon (IFN)- $\alpha$, 
tumour necrosis factor (TNF)- $\alpha$ and interleukin (IL)-12. This cytokine milieu triggers a Th1 cell mediated immune response with generation of cytotoxic effectors cells. These processes are depicted in figure 1.

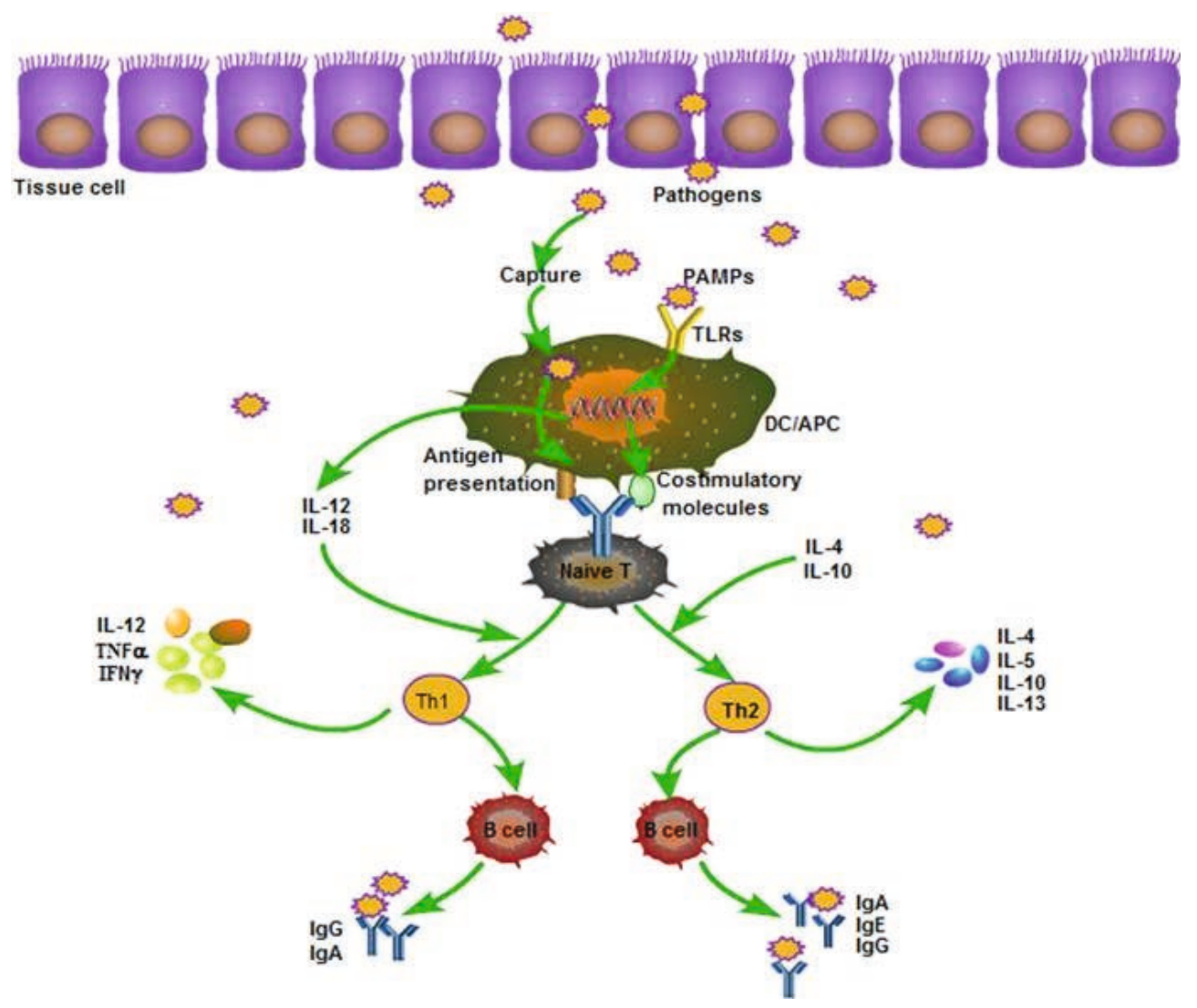

Figure 1. TLRs and T helper cell responses.

PAMPs from invading pathogens bind with TLRs expressed in DCs, and DCs become activated and mature to active naive T cells. Naive T cells are primed toward specific Thelper profiles: Th1, Th2, which can produce cytokines and in turn establish resistance to external pathogen invasion. [49]

In recent years, it has been uncovered that TLRs are also involved in the development of a variety of malignancies.[48, 49] Numerous studies have shown an abnormal expression of TLRs in tumor cells as compared to normal tissue, including in cervical cancer. Through mechanisms not fully understood, TLRs can induce a dysfunctional inflammatory response in a carcinogenic environment. TLR2/3/4/5/9 have been most frequently described in tumor tissues. This dysfunctional inflammatory response contributes to carcinogenesis through several mechanisms: an anti-apoptotic effect through activation of NF-kB, induction of oxidative damage to DNA, activation of the tissue repair response and promotion of angiogenesis through recognition of 
damage-associated molecular patterns (DAMPs). TLRs also play a role in cervical HPV infection and oncogenesis. The viral oncogenes $\mathrm{E} 6$ and $\mathrm{E} 7$ have been shown to inhibit the activity of NF-kB in response to TLR stimulation, thereby reducing antigen presentation. This enables HPV to evade local immune response and enables persistent infection.[48, 49, 51] Additionally, two TLRs seem to play an active role in cervical carcinogenesis. [49] TLR4 and TLR9 were found to be gradually upregulated in progression from cervical dysplasia to cervical cancer and their stimulation may contribute to cervical carcinogenesis through mechanisms described above.

\section{Imiquimod in treatment of high-grade CIN}

Imiquimod is an agonist of TLR7 and TLR8. The general antitumoral effect of imiquimod is mediated through at least three mechanisms. [52] The most important effect is agonistic activity towards TLR7 and -8, which activates NF-kB, inducing cell-mediated immunity. Imiquimod leads to a profound T-helper (Th)-1-weighted cellular immune response. TLR-independent effects are interference with adenosine receptor signaling pathways, which augments proinflammatory activity, and induction of apoptosis at higher concentrations through activation of a mitochondrial pathway. Imiquimod was shown to induce regression of high-grade CIN lesions in a clinical study, but the specific biological mechanisms of imiquimod in CIN lesions have not been completely elucidated.[15] Both TLR7 and TLR8 are found in the epithelium and stroma of cervical epithelium, but expression is limited.[53] One study found a mildly increased expression of TLR7 in CIN and cervical carcinoma as compared to normal epithelium, but this finding was not confirmed in a later study. [53, 54] This does not argue for a significant upregulation of TLR during cervical carcinogenesis, indicating that TLR7/8 are most likely not procarcinogenic in the human cervix. A clinical study indicated that a higher expression of cervical TLR7 in HPV16-positive women was a significant predictor of HPV clearance during follow-up. [55] As such, stimulation of TLR7/8 may contribute to HPV clearance and possibly regression of $\mathrm{CIN}$. More specific evidence regarding the biological mechanisms of imiquimod in HPV induced precancerous lesions is available from studies in VIN. Two immunological studies - in a limited number of women - showed that imiquimod treatment did not lead to a general induction or enhancement of HPV16-specific CD4+ and CD8+ T-cell responses. [56, 57] This indicates an effect of imiquimod other than induction of a specific immune response through TLR $7 / 8$. Interestingly, a stronger preexisting HPV-specific type-1 T-cell response was associated with a more favorable clinical outcome upon topical imiquimod treatment. The authors propose that the efficacy of imiquimod in VIN may be explained by the induction of additional pro-inflammatory signals, through Langerhans cells and macrophages and by stimulation of natural killer cells and Th1 cells via indirect mechanisms. This may restore the aberrant immunological environment that characterizes VIN (and CIN) lesions and allow for lesion regression. Indeed, a later study by the authors showed a normalization of immune cell counts in patients that cleared HPV and showed histologic regression after imiquimod treatment.[58] Additional evidence for a TLR independent effect of imiquimod was found in a cell line study on primary human skin derived keratinocytes. [51] In this study, it was shown that the effects of imiquimod treatment on NF-KB activation and cytokine secretion was independent of TLR $7 / 8$. Furthermore, it was found that undifferentiated primary keratinocytes do not express TLR $7 / 8$. The antitumoral effects of imiquimod in high-grade $\mathrm{CIN}$ may therefore be the result of two simultaneous mechanisms: first, stimulation of TLR7/8 in 
differentiated keratinocytes leading to normalization of the abberant immune response caused by HPV infection, and second, a TLR 7/8 independent pathway that augments pro-inflammatory activity and induces apoptosis as has been described above. Further studies in CIN lesions could unravel the mode of action of imiquimod in regression of high-grade $\mathrm{CIN}$.

\section{Imiquimod in clinical practice}

An inventory among Dutch gynecologists shows that clinical application of imiquimod in highgrade $\mathrm{CIN}$ is currently very limited. We identified three important reasons for this. First, a limited awareness among physicians of imiquimod as a treatment modality in high-grade CIN. Second, a perceived lack of evidence regarding clinical efficacy and recurrence rates. Third, concerns about clinical applicability, in the sense of side effects and treatment intensity.. The study also revealed a (not unexpected) limited area of indication for imiquimod in high-grade $\mathrm{CIN}$ : imiquimod was almost exclusively applied to avoid a secondary LLETZ or primary LLETZ in women with a future pregnancy desire. This perceived area of indication was confirmed in our patient preference study, which clearly indicated that a preference for imiquimod treatment instead of LLETZ is prompted by a future pregnancy desire. Women with a future pregnancy desire accept a lower treatment efficacy and a higher incidence of side effects, in return for a lower risk of obstetric side effects. Nonetheless, a certain minimal treatment efficacy of imiquimod is desired by these women: they prefer imiquimod over LLETZ under the condition that the imiquimod efficacy rate reaches $72 \%$. The only study on imiquimod efficacy by Grimm et al. showed a regression rate of $73 \%$ and a remission rate of $49 \%$.[15] Additional evidence on the long-term treatment efficacy of imiquimod treatment should clarify whether a treatment success rate of at least $72 \%$ is actually achievable in order to make imiquimod treatment worthwhile for women. When this efficacy rate is not reached in the TOPIC-3 study, we suggest the identification of predictive markers for treatment outcome of imiquimod. This would enable selection of those women in whom an adequate response to imiquimod treatment is expected. Biomarkers for imiquimod treatment outcome should ideally reflect plausible biological processes in pathophysiology and pharmacology. Predictive biomarkers for imiquimod treatment outcome could therefore be identified at the level of patient and lesion characteristics (such as smoking status, CIN grade, HPV genotype and lesion size) or on the level of functional cellular mechanisms in the pathogenesis and regression of CIN and pharmacological effects of imiquimod (such as proliferation markers, genetic markers, TLR-7/8 expression and markers reflecting immune responses). Such predictive biomarkers for treatment outcome were previously studied in VIN. Jones et al. studied HPV DNA methylation as a predictor for clinical response of VIN3 to imiquimod and cidofovir.[59] They hypothesized that HPV infection is likely to be most immunogenic in the context of a productive infection, which are associated with low levels of viral DNA methylation. Indeed, they found that median E2 methylation was lower in patients who responded to imiquimod treatment, but the result was not significant. E2 methylation $<4 \%$ predicted adequate treatment response with $70.6 \%$ sensitivity and $62.5 \%$ specificity. [59] As such, E2 methylation does not classify as a valid predictive marker for imiquimod treatment outcome. Markers of the immune response have also been studied with regard to imiquimod treatment outcome. As discussed before, Van Poelgeest et al. found that a preexisting HPV16-specific type 1 T-cell response was associated with a more favorable clinical outcome upon topical imiquimod treatment in VIN in HPV16 positive women. 
[56] Based on these results, differences in local immune response at baseline could influence the outcome of imiquimod treatment. It has been established that CIN lesions are characterized by an increasing aberrant immune response with increasing disease severity. Hypothetically, a more favorable immune response at baseline may reflect either a less advanced lesion or a more capable immune system, potentially making the lesion more sensitive to immunotherapy. A final example of a potential marker of imiquimod treatment outcome may be TLR7/8 expression. Scott et al. showed that a higher expression of TLR7 in HPV16-positive women was a significant predictor of HPV clearance during follow-up. [55] Halec et al. studied TLR expression in relation to regression of $\mathrm{CIN} 2$ lesions and found that women with $\mathrm{CIN} 2$ regression showed significantly higher baseline levels of TLR7 compared to women with CIN2 persistence/progression.[60] Increased expression of TLR7/8 may induce a more effective response to imiquimod treatment in high-grade $\mathrm{CIN}$, making TLR7/8 expression a potential predictive marker in this regard. The quest for predictive biomarkers for imiquimod treatment outcome is part of the TOPIC3 study which is currently being conducted.

\section{The TOPIC-3 study}

Nearly all gynecologists that responded to our survey indicated that they would be willing to apply imiquimod in high-grade $\mathrm{CIN}$, upon additional evidence on treatment efficacy and clinical applicability. This clearly emphasized the need for further studies on imiquimod as a treatment modality for high-grade CIN. The original TOPIC trial was a randomized controlled trial (RCT), designed to assess short- and long-term treatment efficacy, side effects and quality of life of imiquimod treatment compared to observational management and LLETZ treatment. Additionally, we aimed to identify prognostic biomarkers for spontaneous regression in the observational arm. Inclusion of women was first hampered by the observational arm: most women preferred treatment instead of watchful waiting. After removal of the observational arm, inclusion was further hampered by a strong preference of women for either of the treatment modalities. Consequently, the TOPIC trial was preliminary halted and converted to a nonrandomized study, to evaluate the treatment efficacy and clinical applicability of imiquimod cream as compared to LLETZ, in selected populations of women with a preference for either of the two treatment modalities (TOPIC-3 study). The patient inclusions of the TOPIC-3 study have been completed at the time of writing, 4 months prior to the expected completion date.

\section{Conclusion}

This thesis discusses alternative strategies in the treatment of high-grade CIN. Based on the available biomarker research and our assessment of HLA types and the 3926 locus, it is not yet possible to adequately predict the natural history of high-grade CIN lesions in general. In $\mathrm{CIN} 2$, simple clinical biomarkers can give an indication of the individual likelihood of disease regression, aiding shared decision-making regarding treatment options. While observational management of $\mathrm{CIN} 2$ is considered safe and can be applied, adequate treatment modalities for $\mathrm{CIN}_{3}$ remain vital. Imiquimod as a treatment modality for high-grade $\mathrm{CIN}$ may be a suitable alternative to surgical treatment for women who want to refrain from surgery, in order to reduce side effects of surgical excision. 
The TOPIC-3 study should clarify the clinical applicability of imiquimod cream as a treatment modality for high-grade CIN.

\section{Future perspectives}

Future perspectives of the issues addressed in this thesis include further development and implementation of prognostic biomarker profiles or prediction models in high-grade CIN and clarification of the clinical applicability of imiquimod in treatment of high-grade $\mathrm{CIN}$, followed by strategies for actual clinical implementation.

Further biomarker research should be conducted according to the PROBE criteria for biomarker research, in order to improve interpretation, comparison and pooling of study outcomes. Ideally, given the distinct difference in natural history of $\mathrm{CIN} 2$ and $\mathrm{CIN}_{3}$ lesions, prognostic markers should be studied in these groups separately. Previously identified prognostic markers and the markers identified in our studies can be incorporated in future biomarker profiles or prediction models. Hopefully, this leads to the development of biomarker profiles or prediction models with adequate test characteristics for the prediction of disease outcome in $\mathrm{CIN} 2$ and $\mathrm{CIN}_{3}$. This offers the opportunity for an individualized treatment strategy, in which a wait-and-see policy could be applied in those cases in which regression is expected. Women with $\mathrm{CIN} 2 / 3$ may benefit from this strategy, as they will be subject to less overtreatment and associated side effects. Clinical applicability of prognostic markers should then be further evaluated with regard to patient satisfaction with observational treatment and cost-effectiveness of this strategy. Finally, prognostic factors that can be influenced should be more actively addressed with patients. Based on the results of our study in hrHPV positive $\mathrm{CIN} 2$ lesions, which once again shows the important effect of smoking, we suggest that smoking cessation programs should be offered to smoking women with $\mathrm{CIN} 2 / 3$.

The TOPIC-3 study will clarify the clinical applicability of imiquimod cream as a treatment modality for high-grade CIN. It will provide additional evidence on short-and long-term treatment efficacy, side effects and quality of life of imiquimod treatment. Upon the establishment of adequate treatment efficacy and clinical applicability, we advocate the application in women with a future pregnancy desire. Other indications may be large $\mathrm{CIN}$ lesions requiring extensive excision, or recurrent $\mathrm{CIN}$ lesion with the aim to prevent repeated cervical surgery including hysterectomy. The efficacy of imiquimod in recurrent $\mathrm{CIN}$ lesions is currently being studied in the TOPIC-2 trial.[4] The identification of prognostic markers for imiquimod treatment outcome could enable selection of women in whom an adequate response to imiquimod treatment is expected. To this aim, the biological mechanisms behind imiquimod-associated clearance of high-grade CIN should be further elucidated. An intended study by the authors on cervical TLR expression and the immune response to imiquimod in high-grade CIN could contribute to this issue. After confirmation of the efficacy and clinical applicability of imiquimod, the awareness of this treatment modality should be increased among physicians and patients. Awareness among physicians could be achieved by incorporation in treatment guidelines, communication through gynecological associations and education. Awareness among patients could be achieved by 
incorporation of the treatment modality in patient information brochures. Remaining knowledge gaps with regard to imiquimod treatment of high-grade CIN are cost-effectiveness and patient and physician satisfaction with imiquimod treatment. These issues could be addressed in future studies. Finally, long-term studies should reveal whether imiquimod indeed reduces long-term side effects of surgical treatment, with emphasis on premature birth after both treatment modalities. 


\section{References}

1. Ostor AC. Natural history of cervical intraepithelial neoplasia: a critical review. Int ] Gynecol Pathol. 1993;12(2):186-92.

2. Kyrgiou M, Athanasiou A, Kalliala IE], Paraskevaidi M, Mitra A, Martin-Hirsch PP, et al. Obstetric outcomes after conservative treatment for cervical intraepithelial lesions and early invasive disease. Cochrane Database Syst Rev. 2017;11:CD012847.

3. Wright TC, Stoler MH, Behrens CM, Sharma A, Zhang G, Wright TL. Primary cervical cancer screening with human papillomavirus: end of study results from the ATHENA study using HPV as the first-line screening test. Gynecol Oncol. 2015;136(2):189-97.

4. van de Sande AJM, Koeneman MM, Cerestein CG, Kruse A], van Kemenade F], van Beekhuizen $\mathrm{H}$ ]. TOPical Imiquimod treatment of residual or recurrent cervical intraepithelial neoplasia (TOPIC-2 trial): a study protocol for a randomized controlled trial. BMC Cancer. 2018;18(1):655.

5. Reuschenbach M, Seiz M, von Knebel Doeberitz C, Vinokurova S, Duwe A, Ridder R, et al. Evaluation of cervical cone biopsies for coexpression of p16INK4a and Ki67 in epithelial cells. Int ] Cancer. 2012;130(2):388-94.

6. Silva DC, Concalves AK, Cobucci RN, Mendonca RC, Lima PH, Cavalcanti G]. Immunohistochemical expression of p16, Ki67 and p53 in cervical lesions - A systematic review. Pathol Res Pract. 2017;213(7):723-9.

7. Litjens R], Hopman AH, van de Vijver KK, Ramaekers FC, Kruitwagen RF, Kruse A]. Molecular biomarkers in cervical cancer diagnosis: a critical appraisal. Expert Opin Med Diagn. 2013;7(4):365-77.

8. De Strooper LM, Hesselink AT, Berkhof ], Meijer C], Snijders P], Steenbergen RD, et al. Combined CADM1/MAL methylation and cytology testing for colposcopy triage of high-risk HPV-positive women. Cancer Epidemiol Biomarkers Prev. 2014;23(9):1933-7.

9. McCredie MR, Sharples K], Paul C, Baranyai ], Medley G, Jones RW, et al. Natural history of cervical neoplasia and risk of invasive cancer in women with cervical intraepithelial neoplasia 3: a retrospective cohort study. Lancet Oncol. 2008;9(5):425-34.

10. Canfell K, Barnabas R, Patnick ], Beral V. The predicted effect of changes in cervical screening practice in the UK: results from a modelling study. Br ] Cancer. 2004;91(3):530-6.

11. Munk AC, Gudlaugsson E, Ovestad IT, Lovslett K, Fiane B, Hidle B, et al. Interaction of epithelial biomarkers, local immune response and condom use in cervical intraepithelial neoplasia 2-3 regression. Gynecol Oncol. 2012;127(3):489-94.

12. Follen M, Atkinson EN, Schottenfeld D, Malpica A, West L, Lippman S, et al. A randomized clinical trial of 4-hydroxyphenylretinamide for high-grade squamous intraepithelial lesions of the cervix. Clin Cancer Res. 2001;7(11):3356-65.

13. Keefe KA, Schell M], Brewer C, McHale M, Brewster W, Chapman JA, et al. A randomized, double blind, Phase III trial using oral beta-carotene supplementation for women with high-grade cervical intraepithelial neoplasia. Cancer Epidemiol Biomarkers Prev. 2001;10(10):1029-35.

14. Alvarez RD, Conner MG, Weiss H, Klug PM, Niwas S, Manne U, et al. The efficacy of 9-cisretinoic acid (aliretinoin) as a chemopreventive agent for cervical dysplasia: results of a randomized double-blind clinical trial. Cancer Epidemiol Biomarkers Prev. 2003;12(2):114-9. 
15. Grimm C, Polterauer S, Natter C, Rahhal ], Hefler L, Tempfer CB, et al. Treatment of cervical intraepithelial neoplasia with topical imiquimod: a randomized controlled trial. Obstet Gynecol. 2012;120(1):152-9.

16. Carcia F, Petry KU, Muderspach L, Gold MA, Braly P, Crum CP, et al. ZYC101a for treatment of high-grade cervical intraepithelial neoplasia: a randomized controlled trial. Obstet Cynecol. 2004;103(2):317-26.

17. Van Pachterbeke C, Bucella D, Rozenberg S, Manigart Y, Gilles C, Larsimont D, et al. Topical treatment of CIN 2+ by cidofovir: results of a phase II, double-blind, prospective, placebocontrolled study. Gynecol Oncol. 2009;115(1):69-74.

18. Kaufmann AM, Nieland JD, Jochmus I, Baur S, Friese K, Gabelsberger ], et al. Vaccination trial with HPV16 L1E7 chimeric virus-like particles in women suffering from high grade cervical intraepithelial neoplasia (CIN 2/3). Int ] Cancer. 2007;121(12):2794-800.

19. Trimble CL, Piantadosi S, Gravitt P, Ronnett B, Pizer E, Elko A, et al. Spontaneous regression of high-grade cervical dysplasia: effects of human papillomavirus type and HLA phenotype. Clin Cancer Res. 2005;11(13):4717-23.

20. Lee MH, Finlayson S], Gukova K, Hanley G, Miller D, Sadownik LA. Outcomes of Conservative Management of High Grade Squamous Intraepithelial Lesions in Young Women.] Low Cenit Tract Dis. 2018;22(3):212-8.

21. Trimble CL, Morrow MP, Kraynyak KA, Shen X, Dallas M, Yan ], et al. Safety, efficacy, and immunogenicity of VCX-3100, a therapeutic synthetic DNA vaccine targeting human papillomavirus 16 and 18 E6 and E7 proteins for cervical intraepithelial neoplasia 2/3: a randomised, double-blind, placebo-controlled phase 2 b trial. Lancet. 2015;386(10008):207888.

22. Munk AC, Kruse A], van Diermen B, Janssen EA, Skaland I, Gudlaugsson E, et al. Cervical intraepithelial neoplasia grade 3 lesions can regress. APMIS. 2007;115(12):1409-14.

23. Godfrey MAL, Nikolopoulos M, Garner JE, Adib TR, Mukhopadhyay D, Rains JS, et al. Conservative management of cervical intraepithelial neoplasia grade $2(\mathrm{CIN} 2)$ in women under 30 years of age: A cohort study. Eur] Obstet Cynecol Reprod Biol. 2018;228:267-73.

24. Pepe MS, Feng Z, Janes H, Bossuyt PM, Potter JD. Pivotal evaluation of the accuracy of a biomarker used for classification or prediction: standards for study design. ] Natl Cancer Inst. 2008;100(20):1432-8.

25. Moody CA, Laimins LA. Human papillomavirus oncoproteins: pathways to transformation. Nat Rev Cancer. 2010;10(8):550-60.

26. Stanley MA. Epithelial cell responses to infection with human papillomavirus. Clin Microbiol Rev. 2012;25(2):215-22.

27. Wentzensen N, von Knebel Doeberitz M. Biomarkers in cervical cancer screening. Dis Markers. 2007;23(4):315-30.

28. Thomas LK, Bermejo JL, Vinokurova S, Jensen K, Bierkens M, Steenbergen R, et al. Chromosomal gains and losses in human papillomavirus-associated neoplasia of the lower genital tract - a systematic review and meta-analysis. Eur ] Cancer. 2014;50(1):85-98.

29. Baak JP, Kruse A], Garland SM, Skaland I, Janssen EA, Tabrizi S, et al. Combined p53 and retinoblastoma protein detection identifies persistent and regressive cervical high-grade squamous intraepithelial lesions. Am J Surg Pathol. 2005;29(8):1062-6. 
30. Bansal A, Pepe MS. When does combining markers improve classification performance and what are implications for practice? Stat Med. 2013;32(11):1877-92.

31. Tainio K, Athanasiou A, Tikkinen KAO, Aaltonen R, Cardenas ], Hernandes, et al. Clinical course of untreated cervical intraepithelial neoplasia grade 2 under active surveillance: systematic review and meta-analysis. BM]. 2018;360:k499.

32. Massad LS, Einstein MH, Huh WK, Katki HA, Kinney WK, Schiffman M, et al. 2012 updated consensus guidelines for the management of abnormal cervical cancer screening tests and cancer precursors. J Low Cenit Tract Dis. 2013;17(5 Suppl 1):S1-S27.

33. Ismail SM, Colclough AB, Dinnen JS, Eakins D, Evans DM, Gradwell E, et al. Observer variation in histopathological diagnosis and grading of cervical intraepithelial neoplasia. BM]. 1989;298(6675):707-10.

34. Carreon JD, Sherman ME, Guillen D, Solomon D, Herrero R, Jeronimo ], et al. CIN2 is a much less reproducible and less valid diagnosis than $\mathrm{CIN} 3$ : results from a histological review of population-based cervical samples. Int ] Gynecol Pathol. 2007;26(4):441-6.

35. Dalla Palma P, Giorgi Rossi P, Collina G, Buccoliero AM, Chiringhello B, Gilioli E, et al. The reproducibility of CIN diagnoses among different pathologists: data from histology reviews from a multicenter randomized study. Am ] Clin Pathol. 2009;132(1):125-32.

36. Cai B, Ronnett BM, Stoler M, Ferenczy A, Kurman R], Sadow D, et al. Longitudinal evaluation of interobserver and intraobserver agreement of cervical intraepithelial neoplasia diagnosis among an experienced panel of gynecologic pathologists. Am J Surg Pathol.2007;31(12):185460.

37. Steenbergen RD, Snijders PJ, Heideman DA, Meijer C]. Clinical implications of (epi)genetic changes in HPV-induced cervical precancerous lesions. Nat Rev Cancer. 2014;14(6):395-405.

38. Uleberg KE, MunkAC, Brede C, Gudlaugsson E, van Diermen B, Skaland I, et al. Discrimination of grade 2 and 3 cervical intraepithelial neoplasia by means of analysis of water soluble proteins recovered from cervical biopsies. Proteome Sci. 2011;9:36.

39. Fonseca-Moutinho JA. Smoking and cervical cancer. ISRN Obstet Gynecol. 2011;2011:847684.

40. Loopik DL, Doucette S, Bekkers RL, BentleyJR. Regression and Progression Predictors of CIN2 in Women Younger Than 25 Years. J Low Genit Tract Dis. 2016;20(3):213-7.

41. Miyamoto S, Hasegawa J, Morioka M, Hirota Y, Kushima M, Sekizawa A. The association between p16 and Ki67 immunohistostaining and the progression of cervical intraepithelial neoplasia grade 2. Int ] Cynaecol Obstet. 2016;134(1):45-8.

42. Guillaud M, Buys TP, Carraro A, Korbelik ], Follen M, Scheurer M, et al. Evaluation of HPV infection and smoking status impacts on cell proliferation in epithelial layers of cervical neoplasia. PLoS One. 2014;9(9):e107088.

43. Samir R, Asplund A, Tot T, Pekar C, Hellberg D. Tissue tumor marker expression in smokers, including serum cotinine concentrations, in women with cervical intraepithelial neoplasia or normal squamous cervical epithelium. Am ] Obstet Cynecol. 2010;202(6):579 e1-7.

44. Munoz N, Franceschi S, Bosetti C, Moreno V, Herrero R, Smith JS, et al. Role of parity and human papillomavirus in cervical cancer: the IARC multicentric case-control study. Lancet. 2002;359(9312):1093-101. 
45. International Collaboration of Epidemiological Studies of Cervical C. Cervical carcinoma and reproductive factors: collaborative reanalysis of individual data on 16,563 women with cervical carcinoma and 33,542 women without cervical carcinoma from 25 epidemiological studies. Int ] Cancer. 2006;119(5):1108-24.

46. Kim JW, Song SH, Jin CH, Lee JK, Lee NW, Lee KW. Factors affecting the clearance of high-risk human papillomavirus infection and the progression of cervical intraepithelial neoplasia. ] Int Med Res. 2012;40(2):486-96.

47. Jensen KE, Schmiedel S, Norrild B, Frederiksen K, Iftner T, Kjaer SK. Parity as a cofactor for high-grade cervical disease among women with persistent human papillomavirus infection: a 13-year follow-up. Br] Cancer. 2013;108(1):234-9.

48. Husseinzadeh N, Davenport SM. Role of toll-like receptors in cervical, endometrial and ovarian cancers: a review. Gynecol Oncol. 2014;135(2):359-63.

49. Yang X, Cheng Y, Li C. The role of TLRs in cervical cancer with HPV infection: a review. Signal Transduct Target Ther. 2017;2:17055.

50. Stanley MA. Imiquimod and the imidazoquinolones: mechanism of action and therapeutic potential. Clin Exp Dermatol. 2002;27(7):571-7.

51. Richards KH, Wasson CW, Watherston O, Doble R, Blair GE, Wittmann M, et al. The human papillomavirus (HPV) E7 protein antagonises an Imiquimod-induced inflammatory pathway in primary human keratinocytes. Sci Rep. 2015;5:12922.

52. Schon MP, Schon M. Imiquimod: mode of action. Br] Dermatol. 2007;157 Suppl 2:8-13.

53. DeCarlo CA, Rosa B, Jackson R, Niccoli S, Escott NG, Zehbe I. Toll-like receptor transcriptome in the HPV-positive cervical cancer microenvironment. Clin Dev Immunol. 2012;2012:785825.

54. Hasimu A, Ge L, Li QZ, Zhang RP, Guo X. Expressions of Toll-like receptors 3, 4, 7, and 9 in cervical lesions and their correlation with HPV16 infection in Uighur women. Chin $]$ Cancer. 2011;30(5):344-50.

55. Scott ME, Ma Y, Farhat S, Moscicki AB. Expression of nucleic acid-sensing Toll-like receptors predicts HPV16 clearance associated with an E6-directed cell-mediated response. Int ] Cancer. 2015;136(10):2402-8.

56. van Poelgeest MI, van Seters $M$, van Beurden $M$, Kwappenberg KM, Heijmans-Antonissen C, Drijfhout JW, et al. Detection of human papillomavirus (HPV) 16-specific CD4+ T-cell immunity in patients with persistent HPV16-induced vulvar intraepithelial neoplasia in relation to clinical impact of imiquimod treatment. Clin Cancer Res. 2005;11 (14):5273-80.

57. Todd RW, Steele JC, Etherington I, Luesley DM. Detection of CD8+ T cell responses to human papillomavirus type 16 antigens in women using imiquimod as a treatment for high-grade vulval intraepithelial neoplasia. Gynecol Oncol. 2004;92(1):167-74.

58. Terlou A, van Seters M, Kleinjan A, Heijmans-Antonissen C, Santegoets LA, Beckmann I, et al. Imiquimod-induced clearance of HPV is associated with normalization of immune cell counts in usual type vulvar intraepithelial neoplasia. Int ] Cancer. 2010;127(12):2831-40.

59. Jones SEF, Hibbitts S, Hurt CN, Bryant D, Fiander AN, Powell N, et al. Human Papillomavirus DNA Methylation Predicts Response to Treatment Using Cidofovir and Imiquimod in Vulval Intraepithelial Neoplasia 3. Clin Cancer Res. 2017;23(18):5460-8.

60. Halec G, Scott ME, Farhat S, Darragh TM, Moscicki AB. Toll-like receptors: Important immune checkpoints in the regression of cervical intra-epithelial neoplasia 2. Int] Cancer. 2018. 


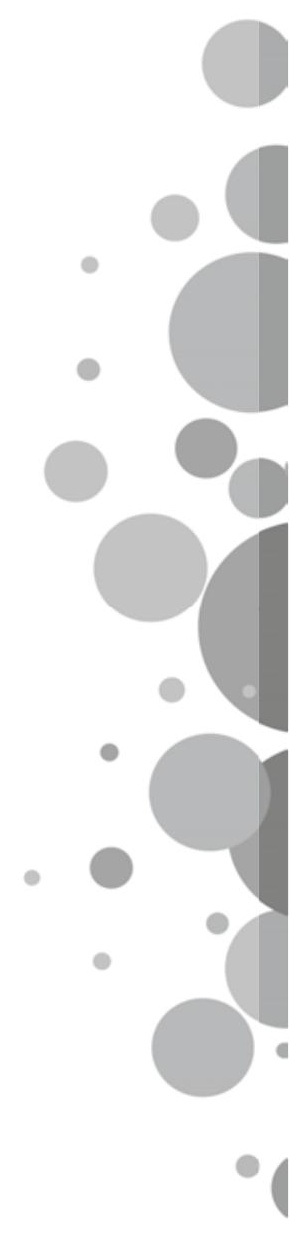




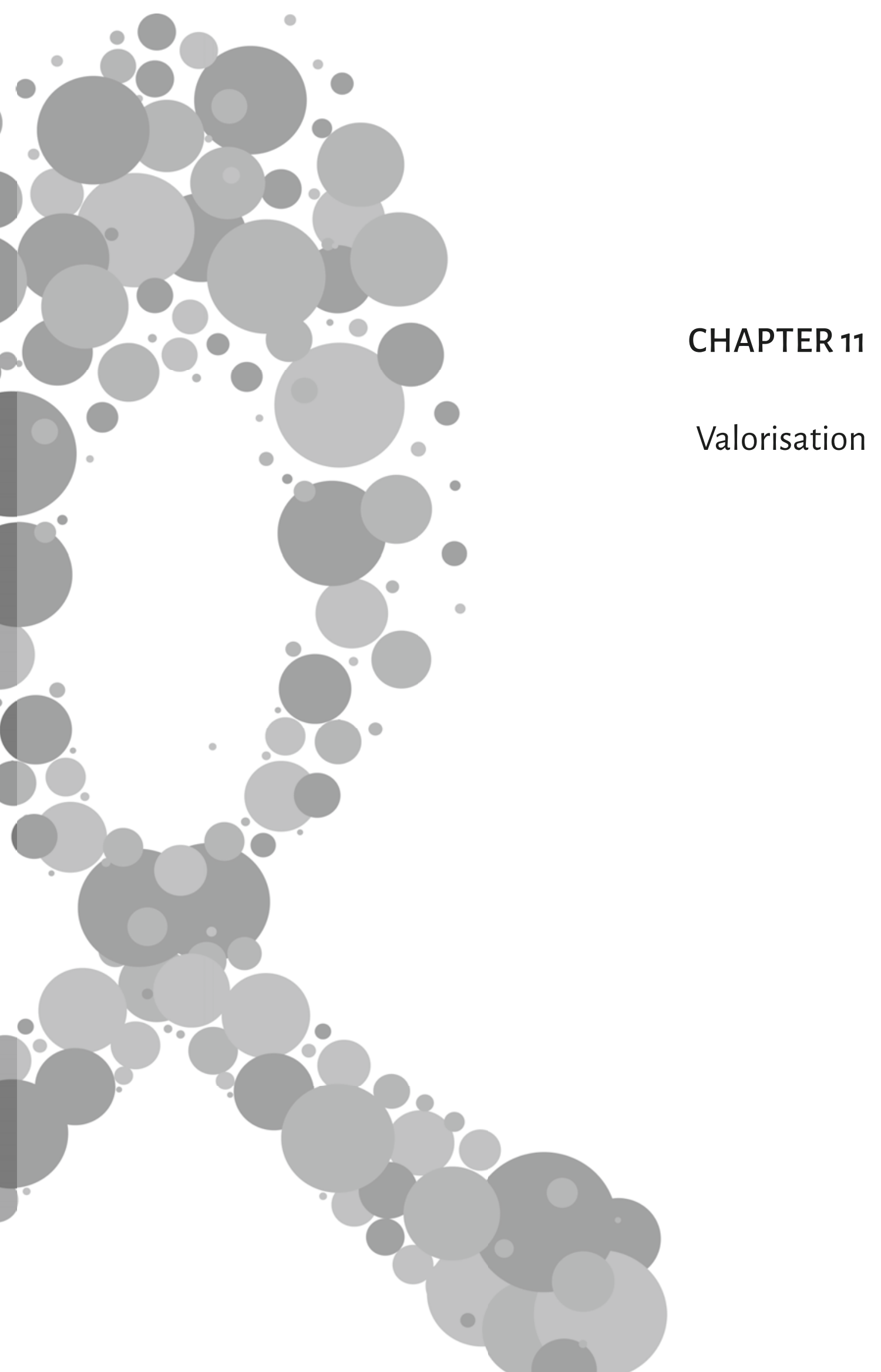





\section{Why is this thesis relevant?}

\section{Relevance of non-invasive management of high-grade $\mathrm{CIN}$}

High-grade Cervical Intraepithelial Neoplasia (CIN2 and CIN3) is the precursor of cervical cancer. Not all high-grade $\mathrm{CIN}$ lesions progress to cervical cancer: this happens in approximately $30 \%$ of lesions, while spontaneous regression occurs in $20-40 \%$ of cases. Because the natural prognosis of an individual lesion cannot be predicted, high-grade CIN lesions were - until recently - all treated by surgical excision. This obviously results in overtreatment of those lesions that would regress spontaneously. In the Netherlands, approximately 5,000 women are treated for highgrade CIN yearly, most commonly by surgical excision of the cervical transformation zone (Large Loop Excision of the Transformation Zone - LLETZ). This is an effective treatment modality, but it is associated with a twofold increase in the risk of premature birth in subsequent pregnancies, probably due to cervical insufficiency. Premature birth, both early and late, has significant impact on neonatal development and parental quality of life. The risk of premature birth after LLETZ treatment could be avoided by non-invasive management of high grade $\mathrm{CIN}$.

\section{The role of prognostic biomarkers in non-invasive management of high-grade CIN}

A first strategy towards non-invasive treatment of high-grade $\mathrm{CIN}$ is observational management of those lesions that will show spontaneous regression. This would reduce overtreatment of high-grade $\mathrm{CIN}$ lesions. Current histopathological assessment is unable to differentiate between lesions that will progress to cervical cancer and those that will regress spontaneously. Instead, prognostic biomarkers could be applied to this aim. A wide variety of prognostic biomarkers has been studied, but none have reached clinical implementation. This thesis provides an overview of all studied prognostic biomarkers in high-grade $\mathrm{CIN}$, which could serve as a general overview and as a basis for further research. Based on this review and our own biomarker studies, we have formulated several recommendations for future biomarker research, which include the application of the PROBE-criteria and the development of biomarker profiles or prediction models rather than individual markers.

We developed one such biomarker profile for spontaneous regression of $\mathrm{CIN}$. Although guidelines recommend observational treatment of $\mathrm{CIN} 2$ in younger women, adherence to this recommendation is not optimal. This could be due to fear of disease progression by both the patient and the physician. Our prediction model can be applied in such cases, to provide patients (and physicians) with a more individual risk prediction. This improves individual counseling of women with $\mathrm{CIN} 2$ and may reassure women that observational treatment is a good option when the chances of disease regression are high. The model consists of simple clinical parameters, instead of expensive or complicated markers, to make it widely applicable. To improve individual counseling of women with hrHPV positive $\mathrm{CIN} 2$, which concerns almost all lesions since the introduction of the new cervical cancer screening program, we identified two prognostic markers in this subgroup of patients. Smoking status and parity influence the natural prognosis of hrHPV positive $\mathrm{CIN} 2$ and these factors can now be taken into account when counseling women with regard to treatment options in hrHPV positive $\mathrm{CIN} 2$. 


\section{The role of imiquimod treatment in non-invasive management of high-grade CIN}

A second strategy towards non-invasive treatment of high-grade CIN is the application of nonsurgical treatment modalities. One non-surgical treatment modality is included in the Dutch treatment guideline for $\mathrm{CIN}$ : imiquimod, a cream with antiviral and antitumor properties. However, evidence regarding treatment efficacy and clinical applicability is scarce and clinical application is very limited. The studies in this thesis provide additional evidence on the desirability and clinical applicability of imiquimod as a treatment modality for high-grade CIN. First, we identified reasons for the currently limited application: this seemed to be the result of a lack of awareness among physicians, but also a perceived lack of evidence regarding treatment efficacy and a high rate of side effects. Subsequently, a patient preference study was conducted to assess the desirability of imiquimod treatment among women. This study indicated a subgroup of women with a future pregnancy wish as potential candidates for imiquimod treatment. These results show that there seems to be an area of indication for imiquimod in treatment of highgrade $\mathrm{CIN}$, but additional evidence on treatment efficacy and side effects is necessary. The TOPIC3 study was designed to provide this evidence. The inclusions of this study have been completed and the results are expected in the spring of 2019. Upon confirmation of its efficacy and tolerability, the results of this study may increase the clinical application of imiquimod as an alternative to LLETZ, with the aim to reduce future premature birth as a side effect of surgical treatment.

\section{Target groups}

The results of this thesis are relevant for gynaecologists, pharmacists, high-grade CIN patients and research groups in the areas of prognostic biomarkers and imiquimod treatment of high-grade $\mathrm{CIN}$. Application and development of prognostic biomarkers may improve individual patient counseling and management, reducing overtreatment of high-grade $\mathrm{CIN}$. When the TOPIC-3 study confirms the clinical applicability of imiquimod as a treatment modality for high-grade $\mathrm{CIN}$, it can be offered to women as an alternative to surgical treatment. Both strategies contribute to a reduction of surgical side effects, with the risk of future preterm birth as the most important.

\section{Activities and innovation}

We have provided a first overview of all previously studied prognostic biomarkers in highgrade $\mathrm{CIN}$ and further investigated two promising markers. These results can function as a tool for future biomarker research. For these studies, we collaborated with our colleagues at the Stavanger University Hospital in Norway, where a database and biopsy material is available from a cohort of women with high-grade CIN who were left untreated for a mean of 16 weeks. We developed a first prediction model for spontaneous regression of $\mathrm{CIN} 2$ and identified for the first time prognostic biomarkers for hrHPV positive CIN2, which may aid physicians in a more individual patient management. For these studies, we collaborated with the department of Clinical Epidemiology and Medical Technology Assessment of the Maastricht University. To assess the clinical applicability of imiquimod treatment, we not only started a clinical study on treatment efficacy and side effects of imiquimod in high-grade $\mathrm{CIN}$, but we also performed an inventory among gynecologists and a patient preference study to assess the support among these 
groups for this treatment modality. This is important in the context of personalized medicine and shared decision-making. The patient preference study was performed in collaboration with the Clinical Epidemiology and Medical Technology Assessment of the Maastricht University. The TOPIC3 study is a national multicenter study, in which we collaborate with the Erasmus MC and the Catharina Ziekenhuis Eindhoven. To generate awareness of our study results, all studies were submitted to international scientific journals and many have been presented at international congresses.

\section{Schedule and implementation}

This thesis provides evidence and tools for non-invasive management strategies in highgrade $\mathrm{CIN}$, focusing on the prediction of spontaneous regression with prognostic biomarkers and immunotherapy with imiquimod. The results of this thesis could lead to the following implementations and future research opportunities.

- We recommend further research on prognostic biomarkers in high-grade CIN. This should be conducted according to the PROBE criteria for biomarker research, in order to improve interpretation, comparison and pooling of study outcomes. We believe that studies should aim for the development of biomarker panels or prediction models, in order to increase prognostic properties. Ideally, given the distinct difference in natural history of $\mathrm{CIN} 2$ and $\mathrm{CIN} 3$ lesions, prognostic markers should be studied in these groups separately.

- $\quad$ The prediction model and individual prognostic markers for spontaneous regression of $\mathrm{CIN}_{2}$ can be applied in clinical care, to improve individual patient counseling with regard to their chances of spontaneous regression. Furthermore, prognostic factors that can be influenced should be more actively addressed with patients. The results of our studies once again show the important effect of smoking. We therefore advocate that smoking cessation programs should be offered to smoking women with $\mathrm{CIN} 2 / 3$.

- The results of the inventory among gynecologists and the patient preference study, which show support for imiquimod as a treatment modality for high-grade $\mathrm{CIN}$ upon confirmation of its efficacy, serve as a justification for the performance of the TOPIC3 study and, hopefully, future clinical implementation of this treatment modality in specific patient groups.

- $\quad$ The TOPIC3 study will clarify the clinical applicability of imiquimod cream as a treatment modality for high-grade CIN. It will provide additional evidence on short- and long-term treatment efficacy, side effects and quality of life of imiquimod treatment. Upon the establishment of adequate treatment efficacy and clinical applicability, the results could support physicians and patients in a choice for imiquimod as treatment for high-grade CIN.

- $\quad$ A second aim of the TOPIC3 study is the identification of predictive markers for adequate treatment outcome. This could enable selection of women in whom an adequate response to imiquimod treatment is expected. To this aim, the biological mechanisms behind imiquimod-associated clearance of high-grade CIN should be further elucidated. An intended study by the authors on cervical TLR expression and the immune response to imiquimod in high-grade $\mathrm{CIN}$ could contribute to this issue.

- $\quad$ Other indications for imiquimod treatment of high-grade $\mathrm{CIN}$ may be large lesions requiring 
extensive excision or recurrent CIN lesion, with the aim to prevent repeated cervical surgery including hysterectomy. The efficacy of imiquimod in recurrent CIN lesions is currently being studied in the TOPIC-2 trial, performed by our research colleagues from the Erasmus MC.

- $\quad$ Remaining knowledge gaps with regard to imiquimod treatment of high-grade CIN are cost-effectiveness and patient and physician satisfaction with imiquimod treatment. These issues could be addressed in future studies. Finally, long-term studies should reveal whether imiquimod indeed reduces long-term side effects of surgical treatment, with emphasis on premature birth after both treatment modalities. 



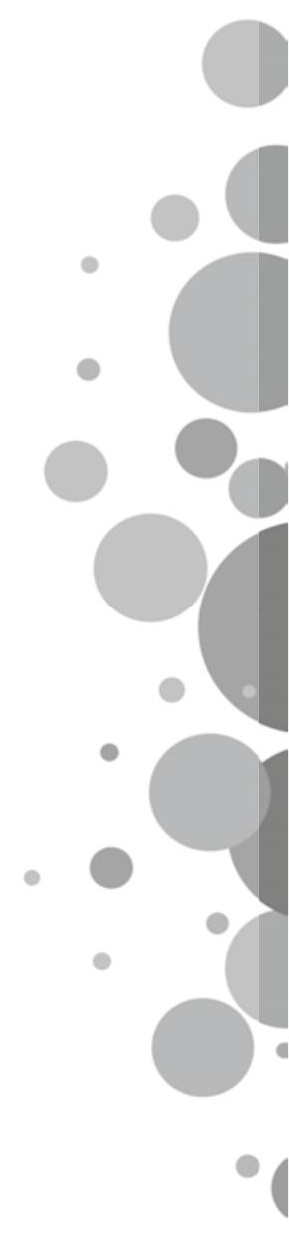




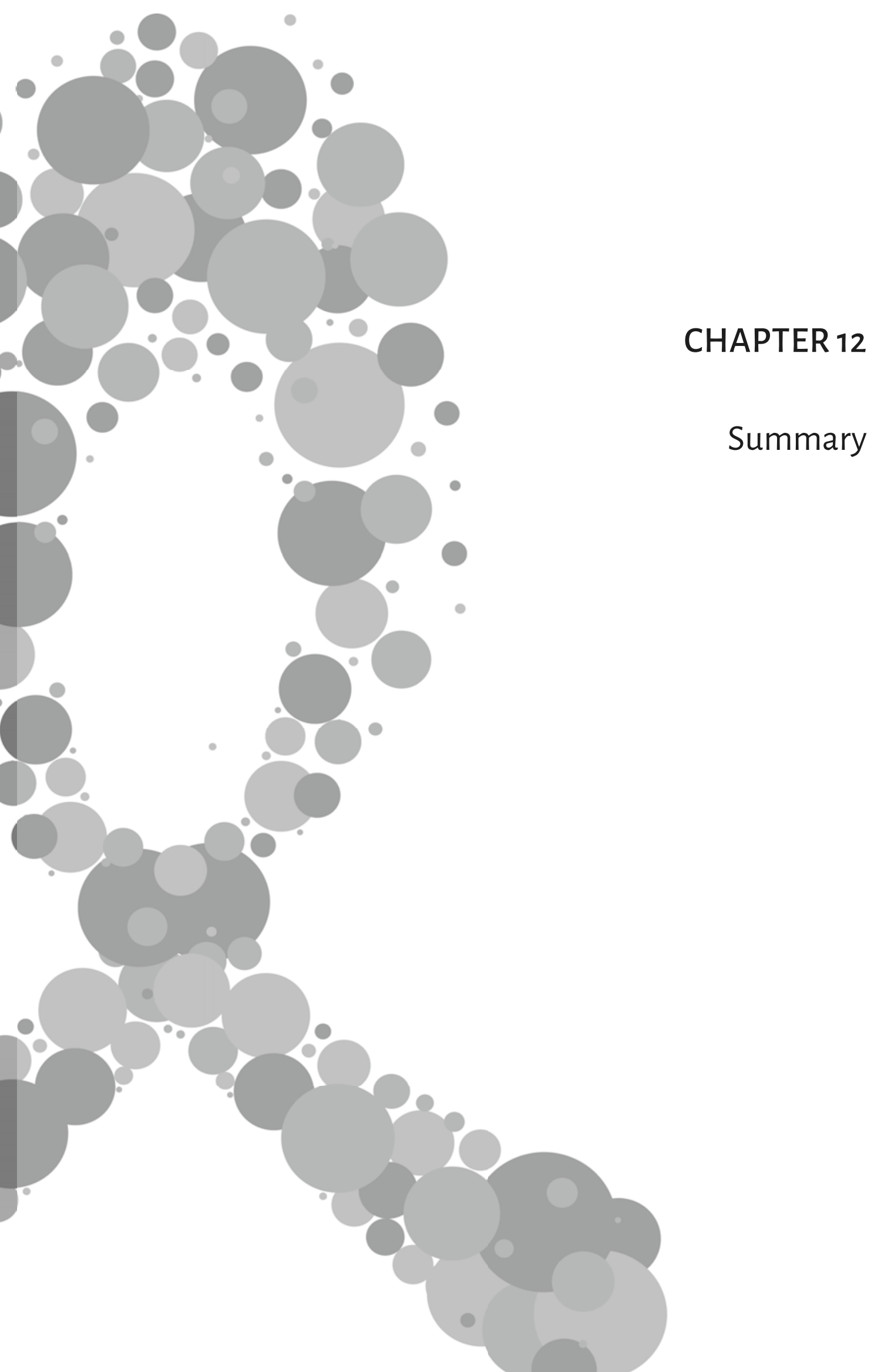



An introduction to the subjects in this thesis is provided in chapter 1. High-grade Cervical Intraepithelial Neoplasia (CIN2 and $\left.\mathrm{CIN}_{3}\right)$ is the precursor of cervical cancer and is caused by cervical infection with the human papillomavirus (HPV). In the Netherlands, approximately 5,000 women are treated for high-grade CIN every year. On the long term, approximately $30 \%$ of high-grade CIN lesions will progress to cervical cancer. On the other hand, 20-40\% would show spontaneous regression when left untreated, but these lesions cannot be identified by current histopathological assessment. Spontaneous regression of $\mathrm{CIN}_{2}$ is even more common: up to $74 \%$ of these lesions are cleared without treatment. Clearance of CIN lesions is performed by the human immune system. Historically, all high-grade CIN lesions were treated by surgical excision, most commonly by Large Loop Excision of the Transformation Zone (LLETZ). This is an effective treatment modality, but is associated with an approximate twofold increased risk of premature birth in subsequent pregnancies. For this reason, guidelines now advice upon observational management of $\mathrm{CIN} 2$ in younger women. Adherence to this recommendation is not optimal, though. $\mathrm{CIN}_{3}$ is still routinely treated with surgical excision, due to its higher potential for malignant evolution. Taken together, a significant number of women is subjected to surgical treatment of high-grade CIN with associated side effects, many of whose lesions would have regressed spontaneously. To reduce overtreatment of CIN, one would ideally be able to predict the disease outcome in individual women to select for observational treatment. Additionally, to reduce side effects of surgical treatment, non-surgical treatment modalities could be applied. Imiquimod, a cream with antiviral and antitumor properties, is the only non-surgical treatment modality currently included in the Dutch treatment guideline for CIN. Clinical application of imiquimod is limited, though, for reasons unknown. Based on these findings, the aims of this thesis were (1) assessment of clinical and molecular biomarkers as predictors of spontaneous regression of high-grade $\mathrm{CIN}$ and (2) assessment of clinical applicability of imiquimod cream as a non-surgical treatment modality for high-grade CIN.

\section{Assessment of clinical and molecular biomarkers as predictors of spontaneous regression of high-grade CIN}

Prognostic biomarkers could be applied to predict the natural disease outcome of high-grade $\mathrm{CIN}$. Cervical oncogenesis and regression of $\mathrm{CIN}$ is the result of a complex interaction between the human immune system, viral factors and functional cellular mechanisms in the cervical epithelium. Biomarkers should be identified at the different levels of this interaction. In chapter $\mathbf{2}$, we therefore first provided an overview of the pathophysiological processes in cervical oncogenesis and regression of CIN. Next, we conducted a review on previously studied prognostic biomarkers in high-grade CIN. None of the included studies was performed according to established criteria for biomarker research (PROBE criteria). We concluded that none of the studied biomarkers are currently fit for clinical implementation, but several promising biomarkers were identified. These include viral genotype and viral DNA methylation (viral factors), HLA-subtypes, markers of lymphoproliferative response, telomerase amplification and HPV-induced epigenetic effects (host factors) and Ki67, p53 and pRb (cellular factors). 
Human Leucocyte Antigens (HLA) alleles were identified a candidate prognostic biomarker for spontaneous regression of high-grade CIN. An individuals' HLA repertoire determines the immune capacity, potentially influencing the capability of $\mathrm{CIN}$ clearance. In chapter 3, we assessed HLA-A, - B, -C, -DRB1 and -DQB1 alleles as prognostic biomarkers in a cohort of 32 women with high-grade $\mathrm{CIN}$, who were followed for a median of 112 days before LLETZ was performed. We found no association was between any HLA allele and spontaneous regression of high-grade CIN. We did find a significant association between the HLA-DRB1"13/14 alleles and HPV status: all patients with these alleles were HPV16-negative, while HPV16 positive lesions did occur in patients without an HLA-DRB1*13/14 allele. HLA-DRB1"13/14 may therefore be protective against the development of HPV16-induced high-grade CIN. This indicates an early and HPV16-specific protective effect of an HLA-DRB1"13/14 epitope.

Gain of the human telomerase RNA gene (hTERC) was also identified as a promising biomarker for disease outcome in high-grade CIN. Overexpression of hTERC allows cells to avoid apoptosis, and thus contributes to carcinogenesis. In chapter $\mathbf{4}$, we assessed whether gain of the chromosomal 3926 region - which contains the hTERC gene -, can predict the natural prognosis of high-grade $\mathrm{CIN}$. To this aim, we first performed a review of the currently available literature. Eight studies were identified, most of which were performed in women with low-grade lesions. All studies found an association between 3926 gain and disease prognosis. Positive predictive values (PPV) ranged from $50-93 \%$, negative predictive values (NPV) ranged from $75-100 \%$. Next, we performed a pilot study in 19 women with high-grade CIN who were followed for a mean of 115 days before LLETZ was performed. 3926 gain was found in 15 lesions, of whom 10 showed disease persistence and 5 showed disease regression. Four lesions showed no 3926 gain, all these lesions regressed. This resulted in a positive predictive value of 3926 gain for disease persistence of $67 \%$, and a negative predictive value of $100 \%$. As such, the absence of 3926 gain is an indicator of disease regression, but the test characteristics of 3926 gain are insufficient for implementation as an individual prognostic biomarker. Further studies could focus on 3926 gain as part of a prognostic biomarker profile, to improve test characteristics.

Treatment guidelines advice upon observational management of $\mathrm{CIN} 2$ lesions in younger women. However, adherence to this recommendation is not optimal. In the era of personalized medicine and shared decision making, a prediction model for spontaneous regression of $\mathrm{CIN}_{2}$ could improve the counseling of women with $\mathrm{CIN} 2$ for observational or invasive treatment. In Chapter 5, we developed such a prediction model. Analysis of 129 women with CIN 2 who were managed by watchful waiting for 6-24 months resulted in a prediction model that consisted of four simple clinical parameters: (1) smoking status, (2) PAP smear result, (3) concomitant $\mathrm{CIN} 1$ in the same biopsy and (4) amount of biopsies containing $\mathrm{CIN} 2$. The area under the curve of the model was $69.2 \%$ [95\% confidence interval $(\mathrm{Cl}), 58.5-79.9 \%$ ], indicating a moderate-to-good discriminative ability of the model. The model proved to be more accurate in prediction of disease regression than disease persistence. The model can thus be applied to reassure patients that observational management is a good option when the model indicates a high probability of regression. As such, this prediction model for spontaneous regression of CIN 2 may aid physicians in the personalized management of these lesions. 
In 2017, a new national screening program for cervical cancer was introduced, based on primary HPV screening instead of primary cytological screening. As a consequence, most newly diagnosed CIN lesions are now high-risk (hr)HPV positive. The prediction model described in the previous chapter was developed in a population of both hrHPV-positive and -negative women, potentially making it less applicable in a population of exclusively hrHPV positive women with $\mathrm{CIN} 2$. Therefore, we aimed to identify prognostic factors for disease outcome in this subgroup of women, in chapter 6 . We analyzed 9 potential prognostic factors in 56 women with hrHPV positive CIN2: HPV16/18 status, p16 staining, KI67 staining, age, smoking status, last PAP smear result, multiple $\mathrm{CIN} 2$ lesions, oral contraception use and parity. Not smoking and nulliparity were identified as significant prognostic factors for disease regression. These factors should be considered in individual patient counseling regarding the choice between immediate treatment or conservative management.

\section{Assessment of clinical applicability of imiquimod cream as a non-surgical treatment modality for high-grade CIN}

In order to assess clinical applicability of imiquimod in CIN, we first evaluated the knowledge, experiences and attitudes of physicians regarding imiquimod treatment of high grade CIN and the preferences of women with high grade $\mathrm{CIN}$ for either surgical or imiquimod treatment. As described in chapter 7, we sent a 37-item questionnaire to all Dutch gynecologists who regularly perform colposcopies. Of the 77 respondents, 58\% were aware of imiquimod for treating CIN. Only five respondents had used imiquimod to treat $\mathrm{CIN}$ and reported generally successful treatment outcomes but frequent side effects. Among all respondents, 96\% stated that they would consider using imiquimod to treat high-grade CIN in selected patients, but only upon additional evidence and inclusion into treatment guidelines. Patient preferences were assessed in chapter 8. A discrete choice experiment (DCE) was conducted among 100 women with abnormal cervical cytology in five hospitals in the Netherlands. Participants were asked to choose between imiquimod treatment or standard surgical treatment in nine separate scenarios, in which the treatment characteristics of both modalities were varied. The DCE showed that most women prefer LLETZ treatment over imiquimod. As expected, the choice for imiquimod treatment was influenced by a future pregnancy desire. Additionally, subgroup analyses revealed women with a future pregnancy desire accept a lower treatment efficacy of imiquimod than women without this desire, but a minimum treatment efficacy of $72 \%$ is still required. We concluded that imiquimod treatment does not seem appropriate for all women with high-grade $\mathrm{CIN}$, but rather for a subgroup of women with a future pregnancy desire, in order to prevent surgical treatment with a risk of premature birth in subsequent pregnancy.

The current recommendation in the Dutch guideline on imiquimod treatment of CIN is based on one RCT. The implementation of a new treatment modality requires robust evidence of its efficacy. Indeed, our survey among gynecologists showed that additional evidence is wanted. For this reason, we designed the TOPIC trial, as described in chapter 9a. This was an RCT, designed to assess short and long-term efficacy of imiquimod treatment in high-grade $\mathrm{CIN}$, as well as side effects and quality of life associated with the treatment modality. The study included three arms: 
an imiquimod treatment arm, a LLETZ treatment arm and an observational arm, in which women were followed for a maximum of 20 weeks with a control colposcopy after 10 weeks. The purpose of this observational arm was to study prognostic biomarkers in high-grade $\mathrm{CIN}$. The inclusion of women into the study was first hampered by this observational arm, which was therefore removed from the study. Thereafter, inclusions were hampered by a strong preference of women for either of the two treatment modalities: most women did not want to be randomized between imiquimod and LLETZ treatment. The study was therefore prematurely terminated, as is described in chapter $\mathbf{9 b}$, and converted to a non-randomized trial (TOPIC-3 study). In this study, women could choose the treatment modality of their preference. In addition to treatment efficacy, sideeffects and quality of life, the TOPIC-3 also studies predictive biomarkers for treatment outcome of imiquimod. This would enable identification of women in whom an adequate response to imiquimod is expected. The TOPIC-3 study is currently running in three centers in the Netherlands. All 120 women have been included at the time of writing. The first study results are expected in the spring of 2019.

In chapter 10, the findings of this thesis are discussed in a general perspective and with regard to their implications for clinical care and future research. This thesis provides evidence and tools for alternative management strategies in high-grade $\mathrm{CIN}$, focusing on the prediction of spontaneous regression with prognostic biomarkers and immunotherapy with imiquimod.

\section{Conclusions, recommendations and future perspectives}

- Prognostic biomarker research is challenging, but short-term observational studies do provide an opportunity for further research in this field. Future prognostic biomarker research should focus on the development of biomarker panels or prediction models, instead of individual markers, and should be performed according to the PROBE criteria for biomarker research.

- $\quad$ Future prognostic biomarker research should distinguish between CIN2 and CIN3 lesions, given the distinct difference in spontaneous regression rates. This distinction should be based on conventional histopathological assessment, until better methods are available.

- $\quad 3926$ gain, smoking status and parity could be considered as part of a biomarker profile or prediction model for spontaneous regression of high-grade $\mathrm{CIN}$.

- Based on physician's opinions and patient preferences, imiquimod qualifies as a non-surgical treatment alternative to LLETZ treatment, mainly for women with a future pregnancy desire, to avoid the risk of premature birth in subsequent pregnancies caused by conventional surgical treatment.

- $\quad$ Additional evidence on the clinical applicability of imiquimod in high-grade CIN (including treatment efficacy and side effects) is necessary and will be provided by the TOPIC3 study. 



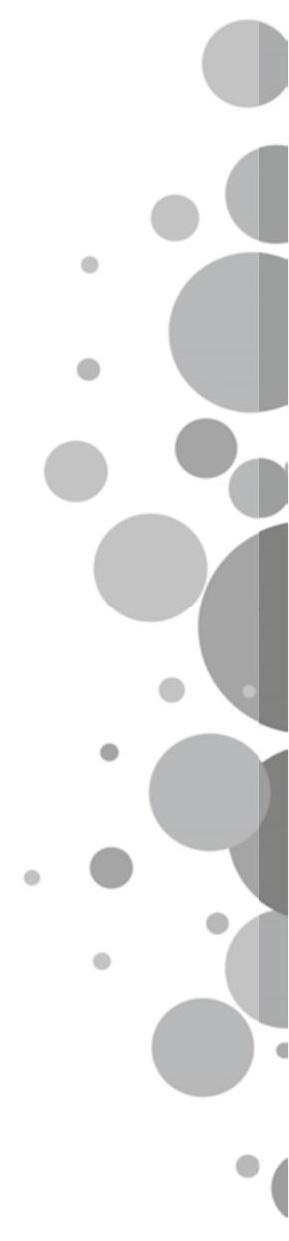




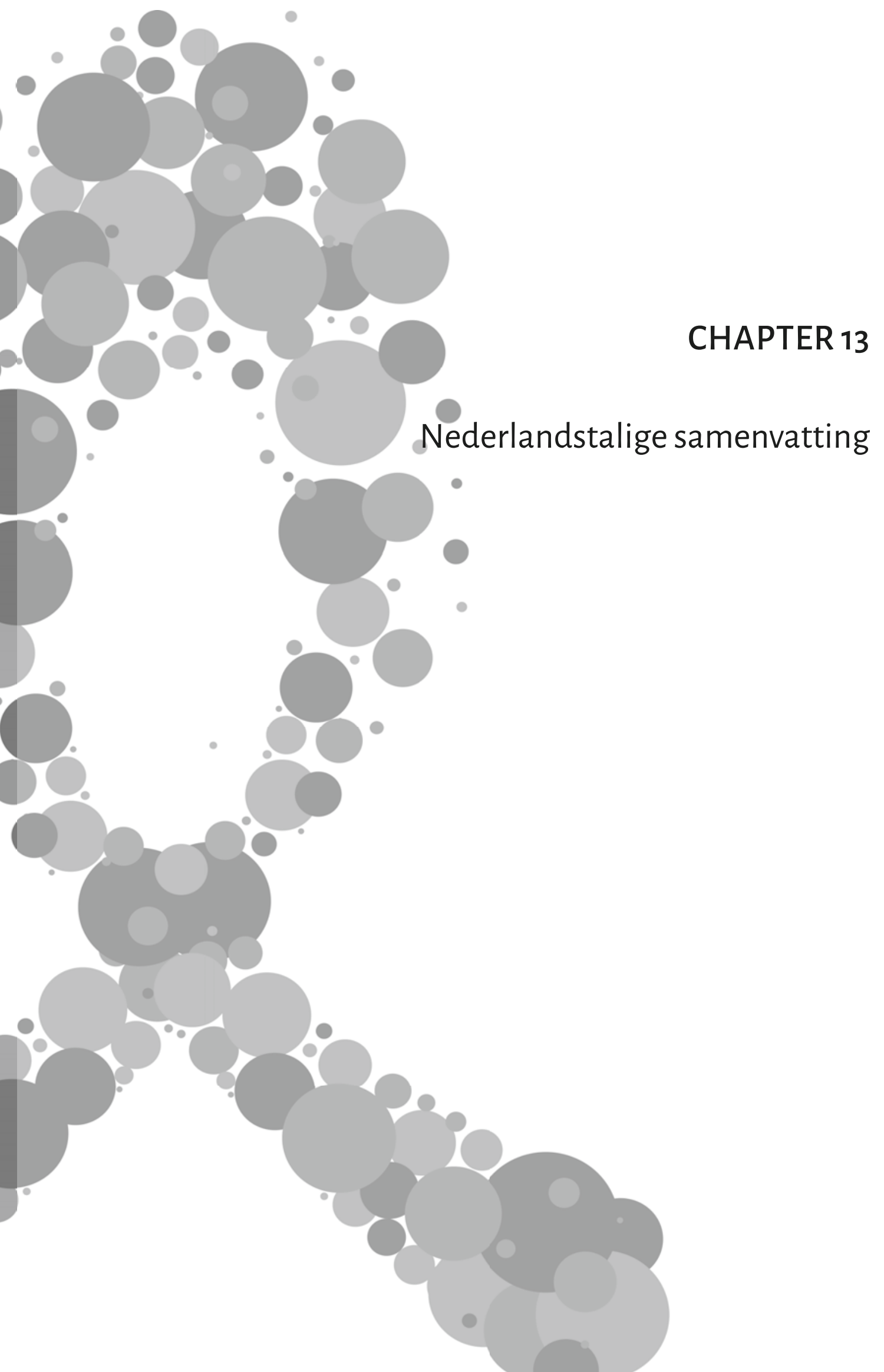



Hoofdstuk 1 is een introductie op de onderwerpen van dit proefschrift. Hooggradige Cervicale Intraepitheliale Neoplasie (CIN2 en CIN3) is een voorloperstadium van baarmoederhalskanker en wordt veroorzaakt door een infectie met het humaan papillomavirus (HPV). In Nederland worden jaarlijks ongeveer 5000 vrouwen behandeld voor een hooggradige CIN. Op de lange termijn ontwikkelt ongeveer 30\% van de hooggradige $\mathrm{CIN}$ afwijkingen zich tot baarmoederhalskanker. Aan de andere kant vertoont 20-40\% van de afwijkingen spontane regressie zonder behandeling. Op basis van het standaard histopathologisch onderzoek kunnen deze afwijkingen echter niet worden onderscheiden van afwijkingen die niet in regressie gaan. Spontane regressie van alleen $\mathrm{CIN} 2$ komt nog vaker voor: tot $74 \%$ van deze afwijkingen verdwijnt zonder behandeling. Regressie van CIN afwijkingen vindt plaats onder invloed van het immuunsysteem. Tot voor kort werden alle hooggradige CIN afwijkingen chirurgisch behandeld, meestal met een lisexcisie (ook wel genoemd: Large Loop Excision of the Transformation Zone-LLETZ). Dit is een effectieve behandeling, maar deze is geassocieerd met een verdubbeld risico op vroeggeboorte in een volgende zwangerschap. Om deze reden wordt in nationale en internationale richtlijnen nu een observationeel beleid geadviseerd bij jonge vrouwen met CIN2. Deze aanbeveling wordt echter niet altijd opgevolgd. CIN3 wordt nog steeds routinematig behandeld met een lisexcisie, gezien het grotere risico op progressie naar kanker. Al met al wordt een behoorlijk aantal vrouwen blootgesteld aan chirurgische behandeling van hooggradige CIN met bijbehorende bijwerkingen en risico's, terwijl een groot deel van hun afwijkingen spontaan zou verdwijnen zonder behandeling. Om deze overbehandeling te voorkomen zou het natuurlijke beloop van hooggradige $\mathrm{CIN}$ idealiter voorspelbaar zijn, om vrouwen met een grote kans op regressie te selecteren voor observationeel beleid. Bij vrouwen voor wie behandeling toch nodig is, zouden bijwerkingen van de lisexcisie kunnen worden voorkomen door het toepassen van een niet-chirurgische behandeling. Imiquimod, een crème werkzaam tegen virussen en bepaalde tumorcellen, is de enige niet-chirurgische behandeling die momenteel is opgenomen in de Nederlandse behandelrichtlijn voor CIN. De klinische toepassing van imiquimod is echter beperkt. Bovenstaande kennis en overwegingen hebben geleid tot de volgende doelstellingen van dit proefschrift: (1) evaluatie en identificatie van klinische en moleculaire biomarkers als voorspellers van spontane regressie van hooggradige $\mathrm{CIN}$ en (2) bepalen van de klinische toepasbaarheid van imiquimod crème in de behandeling van hooggradige $\mathrm{CIN}$.

\section{Klinische en moleculaire biomarkers als voorspellers van spontane regressie van hooggradige CIN}

Prognostische biomarkers zouden kunnen worden toegepast om het natuurlijke beloop van hooggradige $\mathrm{CIN}$ te voorspellen. De ontwikkeling en regressie van CIN wordt bepaald door een complexe interactie tussen het menselijke immuunsysteem, virale factoren en functionele cellulaire mechanismen in het cervicale epitheel. Biomarkers moeten worden gezocht in de verschillende gebieden van deze interactie. In hoofdstuk $\mathbf{2}$ wordt daarom eerst een overzicht gegeven van de pathofysiologische processen in de ontwikkeling en regressie van $\mathrm{CIN}$. Vervolgens wordt een review beschreven van eerder bestudeerde prognostische biomarkers. Geen enkele van de geïncludeerde studies werd verricht volgens de bestaande criteria voor biomarker onderzoek (PROBE criteria). Wij concludeerden dat geen enkele bestudeerde biomarker momenteel 
geschikt is voor toepassing in de dagelijkse praktijk. Er werd wel een aantal veelbelovende markers geïdentificeerd, namelijk: het virale genotype en methylering van het virale DNA (virale factoren), HLA subtypen, markers van de lymfoproliferatieve respons, telomerase amplificatie en epigenetische factoren (gastvrouw factoren), en immunohistochemische expressie van Ki67, p53 en $\mathrm{pRb}$ (cellulaire factoren).

Humane leukocyten antigenen (HLA) allelen werden door ons geïdentificeerd als mogelijke prognostische biomarkers voor spontane regressie van hooggradige $\mathrm{CIN}$. De samenstelling van de HLA allelen bepalen de capaciteit van het individuele immuunsysteem, waardoor mogelijk de spontane regressie van hooggradige CIN wordt beïnvloed. In hoofdstuk 3 onderzochten we de prognostische waarde van de HLA-A, -B, -C, -DRB1 en -DQB1 allelen in 32 vrouwen met hooggradige $\mathrm{CIN}$, die na een observationele periode van gemiddeld 112 dagen werden behandeld met een lisexcisie. We vonden geen associatie tussen HLA allelen en spontane regressie van hooggradige CIN. We vonden wel een significante associatie tussen HLA-DRB1"13/14 allelen en HPV status: alle vrouwen met deze allelen waren HPV16 negatief, terwijl HPV16 positieve CIN wel voorkwam bij vrouwen zonder deze allelen. HLA-DRB1*13/14 zou daarom kunnen beschermen tegen de ontwikkeling van HPV16 geassocieerde hooggradige CIN. Dit wijst op een vroeg en HPV16 specifiek beschermend effect van HLA- DRB1*13/14.

Chromosomale winst van het Humaan Telomerase RNA gen (hTERC) werd ook geïdentificeerd als mogelijke prognostische biomarker in hooggradige CIN. Toegenomen expressie van hTERC door chromosomale winst voorkomt celdood en draagt daarmee bij aan de ontwikkeling van kanker. In hoofdstuk 4 onderzochten we of chromosomale winst van de 3926 regio, waarop het hTERC zich bevindt, de prognose van hooggradige CIN kan voorspellen. We verrichtten eerst een review van alle reeds beschikbare studies op dit gebied. Er werden acht studies gevonden, waarvan de meeste echter waren uitgevoerd in vrouwen met (grotendeels) laaggradige afwijkingen. Alle studies vonden een associatie tussen 3926 winst en de ziekte-uitkomst. Positief voorspellende waarden varieerden van $50-95 \%$, negatief voorspellende waarden varieerden van $75-100 \%$. Vervolgens voerden we een pilot study uit in 19 vrouwen met een hooggradige $\mathrm{CIN}$, die na een observationele periode van gemiddeld 115 dagen werden behandeld met een lisexcisie. Chromosomale winst van 3926 werd gevonden in 15 afwijkingen, waarvan er 10 in regressie gingen en 5 persisteerden. Vier afwijkingen vertoonden geen 3926 winst, al deze afwijkingen gingen in regressie. Deze bevindingen resulteren in een positief voorspellende waarde voor het persisteren van de afwijking van $67 \%$ en een negatief voorspellende waarde van $100 \%$. De afwezigheid van 3926 winst zou dus een prognostische factor kunnen zijn voor spontane regressie. Echter, de testkarakteristieken van 3926 winst zijn onvoldoende voor implementatie ervan als een individuele prognostische factor. In vervolgstudies zou 3926 expressie daarom kunnen worden onderzocht als onderdeel van een biomarker profiel.

In veel behandelrichtlijnen wordt geadviseerd om $\mathrm{CIN} 2$ bij jongere vrouwen niet direct te behandelen, maareersteen observationeel beleid tevoeren. Denalevingvan ditadvies isechterniet optimaal. In een tijd waarin "gepersonaliseerde geneeskunde" en "gedeelde besluitvorming" een belangrijke rol spelen, kan een predictiemodel voor spontane regressie van $\mathrm{CIN} 2$ de counseling 
van individuele vrouwen met $\mathrm{CIN} 2$ verbeteren, met betrekking tot de keuze tussen wel of niet direct behandelen. In hoofdstuk $\mathbf{5}$ hebben we een dergelijk predictiemodel ontwikkeld, op basis van 129 vrouwen met $\mathrm{CIN} 2$ die niet primair behandeld waren, maar zijn opgevolgd gedurende 6-24 maanden. Dit resulteerde in een predictiemodel dat bestaat uit vier simpele klinische parameters: (1) roken, (2) uitslag van het laatste uitstrijkje, (3) gelijktijdige $\mathrm{CIN} 1$ in hetzelfde biopt en (4) het aantal biopten met CIN2. De area-under-the-curve van het model was $69.2 \%$ [95\% confidence interval 58.5-79.9\%], hetgeen wijst op een gemiddeld-tot-goede voorspellende waarde van het model. De voorspellende waarde van het model voor regressie is beter dan de voorspellende waarde voor persisteren. Wanneer het model een hoge kans op regressie aangeeft, kan het dus gebruikt worden om de keuze voor een observationeel beleid te onderbouwen. Op deze manier kan het predictiemodel bijdragen aan een meer gepersonaliseerde counseling van vrouwen met $\mathrm{CIN} 2$.

In 2017 werd een nieuw nationaal bevolkingsonderzoek voor baarmoederhalskanker geïntroduceerd, dat is gebaseerd op primaire HPV screening in plaats van primair cytologisch onderzoek van het uitstrijkje. Als gevolg hiervan zijn vrijwel alle gediagnosticeerde $\mathrm{CIN} 2$ afwijkingen nu hoogrisico (hr)HPV positief. Het predictiemodel dat werd beschreven in het vorige hoofdstuk werd ontwikkeld in een groep vrouwen met zowel hrHPV positieve als negatieve $\mathrm{CIN} 2$, waardoor het mogelijk niet van toepassing is op vrouwen met enkel hrHPV positieve CIN2. Het doel van de studie in hoofdstuk 6 was daarom het identificeren van prognostische factoren voor regressie van $\mathrm{CIN} 2$ in deze specifieke groep vrouwen. We analyseerden 9 potentieel prognostische factoren in 56 vrouwen met een hrHPV positieve CIN2: HPV16/18 status, p16 kleuring, KI67 kleuring, leeftijd, roken, uitslag van het laatste uitstrijkje, meerdere $\mathrm{CIN} 2$ afwijkingen, gebruik van orale anticonceptiepil en pariteit (aantal bevallingen). Niet-roken en nullipariteit (geen bevallingen) bleken significante prognostische factoren voor regressie van hrHPV positieve CIN2. Deze factoren moeten worden meegenomen in de counseling van vrouwen met hrHPV positieve $\mathrm{CIN} 2$, met betrekking tot de keuze tussen directe behandeling of observationeel beleid.

\section{Klinische toepasbaarheid van imiquimod crème in de behandeling van hooggradige $\mathrm{CIN}$}

Om de klinische toepasbaarheid van imiquimod in CIN te bepalen, werd allereerst de kennis, ervaringen en meningen van gynaecologen ten aanzien van deze behandeling geïnventariseerd. Ook onderzochten wij de voorkeuren van vrouwen met hooggradige $\mathrm{CIN}$ voor behandeling met lisexcisie of imiquimod. In hoofdstuk 7 worden de resultaten beschreven van een vragenlijst die werd verstuurd naar alle Nederlandse gynaecologen die regelmatig kolposcopieën uitvoeren. Van de 77 respondenten was $58 \%$ op de hoogte van imiquimod als behandeloptie voor hooggradige $\mathrm{CIN}$. Slechts vijf respondenten hadden het wel eens toegepast voor deze indicatie. Zij rapporteerden over het algemeen positieve behandelresultaten, maar daarbij wel veel bijwerkingen. Van alle respondenten gaf $96 \%$ aan een behandeling van $\mathrm{CIN}$ met imiquimod te willen overwegen in geselecteerde vrouwen, maar alleen wanneer er meer bewijs voor de werkzaamheid zou komen en als de behandeloptie werd opgenomen in de landelijke richtlijn. 
De behandelvoorkeuren van vrouwen met hooggradige CIN werden onderzocht in hoofdstuk 8. Er werd een discrete choice experiment (DCE) uitgevoerd in 100 vrouwen met een afwijkend uitstrijkje in 5 ziekenhuizen in Nederland. Deze vrouwen werd gevraagd om in negen verschillende scenario's te kiezen tussen behandeling met imiquimod of lisexcisie. In elk scenario wisselden de karakteristieken van de behandeling (o.a. effectiviteit en bijwerkingen). Uit dit experiment bleek dat de meeste vrouwen een voorkeur hebben voor een lisexcisie. Zoals verwacht werd de keuze voor imiquimod beïnvloed door een kinderwens in de toekomst. Een subgroep analyse van vrouwen met een toekomstige kinderwens liet zien dat zij een lagere behandeleffectiviteit accepteren dan vrouwen zonder kinderwens in de toekomst, maar dat de effectiviteit wel minstens $72 \%$ dient te zijn. De conclusie van het onderzoek was dat behandeling met imiquimod niet geïndiceerd is voor alle vrouwen met hooggradige $\mathrm{CIN}$, maar juist voor een subgroep van vrouwen met een toekomstige kinderwens, om daarmee het risico op vroeggeboorte in opvolgende zwangerschappen te beperken.

De Nederlandse behandelrichtlijn voor $\mathrm{CIN}$ adviseert om imiquimod toe te passen bij vrouwen met CIN 2-3 die niet primair chirurgisch behandeld willen worden. Dit advies is gebaseerd op de resultaten van slechts één gerandomiseerd onderzoek. De implementatie van een nieuwe behandelmodaliteit vereist robuust bewijs voor de effectiviteit ervan. Uit hoofdstuk 7 bleek inderdaad dat veel gynaecologen aanvullend bewijs voor de effectiviteit als voorwaarde stellen voor de klinische toepassing ervan. Om deze reden werd de TOPIC trial gestart, zoals beschreven in hoofdstuk 9a. Dit was een gerandomiseerd onderzoek, dat als doel had om de effectiviteit op korte en lange termijn van imiquimod in hooggradige $\mathrm{CIN}$ te onderzoeken, alsmede bijwerkingen en de kwaliteit van leven tijdens en na de behandeling. De studie bestond uit drie armen: een groep werd behandeld met een lisexcisie, een groep werd behandeld met imiquimod en in de derde groep ondergingen vrouwen een observationeel beleid gedurende maximaal 20 weken. Het doel van deze laatste arm was om prognostische biomarkers voor spontane regressie van hooggradige $\mathrm{CIN}$ te onderzoeken. De inclusie van vrouwen in de studie werd eerst beperkt door deze observationele arm, die daarom werd verwijderd uit de studie. Daarna bleek echter dat vrouwen niet gerandomiseerd wilden worden tussen de twee verschillende behandelingen, omdat zij over het algemeen een zeer sterke voorkeur hadden voor een van beide behandelingen. Om deze reden werd de studie vroegtijdig gestopt, zoals beschreven in hoofdstuk $\mathbf{9 b}$, en gewijzigd naar een niet-gerandomiseerd onderzoek (TOPIC-3 studie). In dit onderzoek konden vrouwen zelf kiezen voor behandeling met imiquimod of lisexcisie. Naast effectiviteit, bijwerkingen en kwaliteit van leven, onderzoekt deze studie ook voorspellende factoren voor de uitkomst van behandeling met imiquimod, om daarmee vrouwen te kunnen selecteren waarbij een goed effect wordt verwacht. De TOPIC-3 studie wordt momenteel uitgevoerd in drie ziekenhuizen in Nederland (MUMC+, Erasmus MC en Catharina Ziekenhuis). Op het moment van schrijven zijn alle 120 vrouwen geïncludeerd in de studie. De eerste resultaten worden verwacht in het voorjaar van 2019. 
In hoofdstuk 10 werden de bevindingen van dit proefschrift bediscussieerd in een breder perspectief, met bespreking van de implicaties voor klinische zorg en toekomstig onderzoek. Dit proefschrift biedt handvatten voor de verdere ontwikkeling van alternatieve behandelstrategieën in hooggradige $\mathrm{CIN}$, met focus op het voorspellen van spontane regressie met prognostische biomarkers en immunotherapie met imiquimod.

\section{Conclusies, aanbevelingen en toekomstperspectieven}

- $\quad$ Prognostisch biomarker onderzoek is uitdagend, maar korte-termijn observationele studies bieden wel degelijk een mogelijkheid voor onderzoek op dit gebied. Toekomstig biomarker onderzoek zou zich moeten richten op de ontwikkeling van samengestelde biomarker profielen of predictiemodellen, in plaats van op individuele markers, en zou moeten worden verricht volgens de PROBE criteria voor biomarker onderzoek.

- $\quad$ In toekomstig biomarker onderzoek zou onderscheid gemaakt moeten worden tussen $\mathrm{CIN}_{2}$ en $\mathrm{CIN} 3$, omdat de kans op regressie duidelijk verschilt. Dit onderscheid moet gemaakt worden op basis van conventionele histopathologische beoordeling, tot betere methoden beschikbaar zijn.

- Toegenomen expressie van 3q26, roken en pariteit kunnen worden overwogen als onderdeel van een biomarker profiel voor de spontane regressie van hooggradige CIN.

- $\quad$ Uit de meningen van gynaecologen en voorkeuren van patiënten blijkt dat imiquimod toegepast zou kunnen worden als niet-chirurgische behandeling in een selecte groep vrouwen, namelijk vooral bij vrouwen met een toekomstige kinderwens om zo het risico op vroeggeboorte in een volgende zwangerschap door een lisexcisie te voorkomen.

- $\quad$ Aanvullend bewijs voor de klinische toepasbaarheid van imiquimod in hooggradige CIN is noodzakelijk en zal worden geleverd door de TOPIC3 studie. 


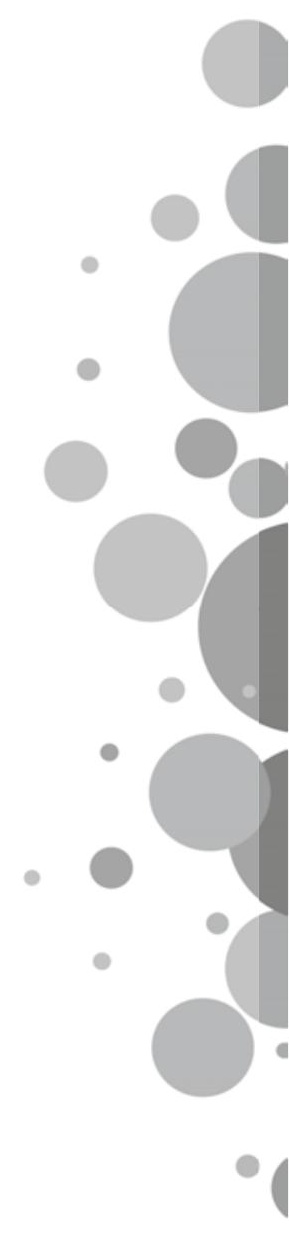




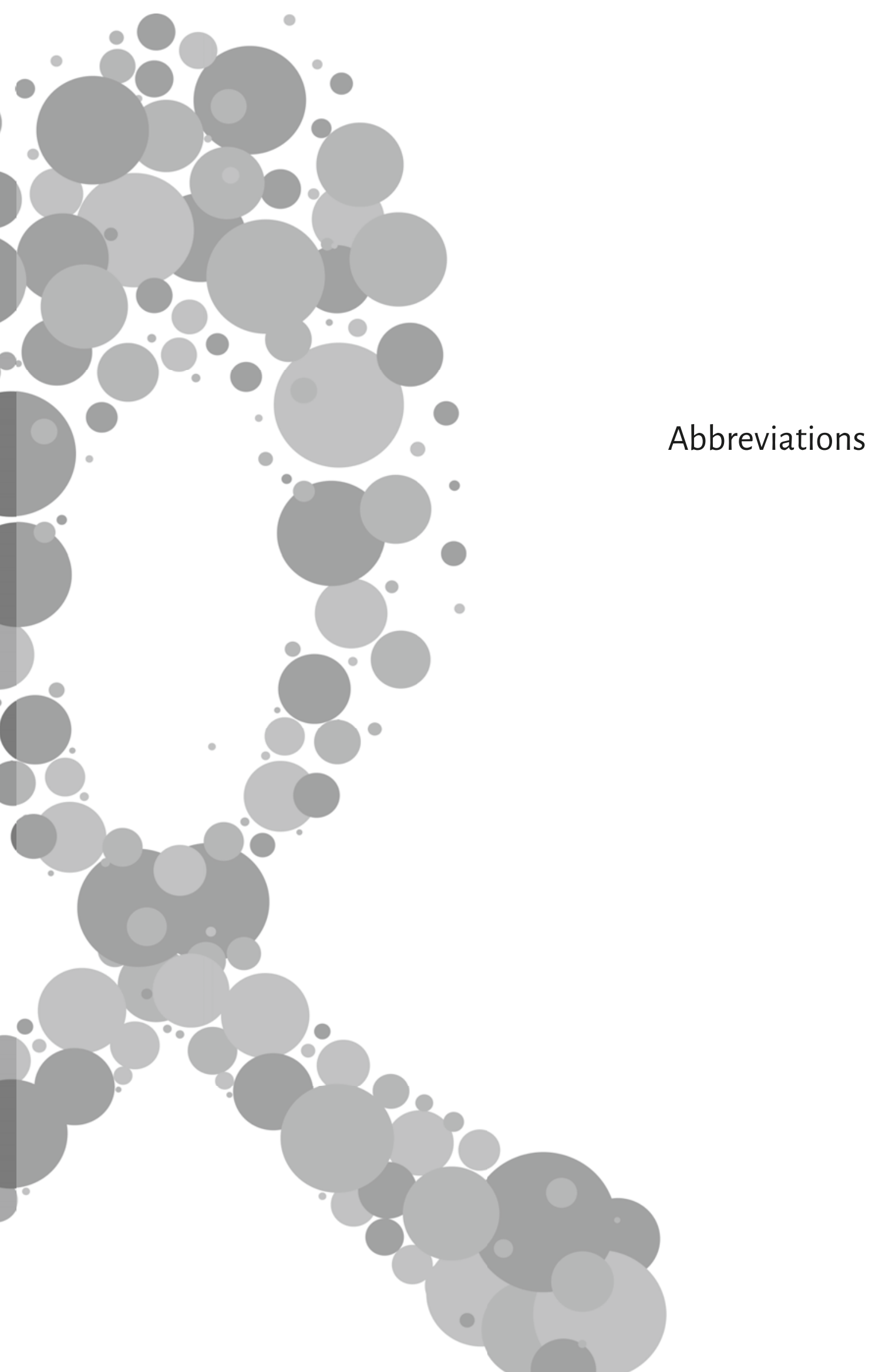




\section{Abbreviations}

\begin{tabular}{|c|c|}
\hline 5 -FU & 5-fluorouracil \\
\hline APC & Antigen presenting cell \\
\hline ASC & Alternative specific constant \\
\hline ASCUS & Atypical squamous cells of undetermined significance \\
\hline AUC & Area under the receiver operation characteristic curve \\
\hline $\mathrm{CADM}_{1} / \mathrm{MAL}$ & Cell adhesion molecule $1 /$ T-lymphocyte maturation assocatiated protein \\
\hline$C D$ & Cluster of differentiation \\
\hline CHERRIES & Checklist for reporting results of internet E-surveys \\
\hline $\mathrm{Cl}$ & Confidence interval \\
\hline $\mathrm{CIN}$ & Cervical intraepithelial neoplasia \\
\hline CK & Cytokeratin \\
\hline $\mathrm{CpC}$ & Cytosin-phosphatidic-Guanin \\
\hline CRF & Case report form \\
\hline CTL & Cytotoxic T cells \\
\hline DAMP & Damage-associated molecular pattern \\
\hline DC & Dendritic cell \\
\hline DCE & Discrete choice experiment \\
\hline DNA & Deoxyribonucleic acid \\
\hline EDTA & Ethylene diamine tetraacetic acid \\
\hline EORTC & European organization for research and treatment of cancer \\
\hline $\mathrm{FISH}$ & Fluorescence in situ hybridization \\
\hline HES & Hematoxylin erythrosin saffran \\
\hline HLA & Human Leucocyte Antigen \\
\hline HPV & Human papillomavirus \\
\hline hrHPV & High-risk human papillomavirus \\
\hline HSIL & High grade squamous intraepithelial lesion \\
\hline hTERC & Human telomerase RNA gene \\
\hline IFN & Interferon \\
\hline IL & Interleukin \\
\hline $\mathrm{IMP}_{3}$ & Insulin-like growth factor II m-RNA-binding protein 3 \\
\hline LEEP & Loop electrosurgical excision procedure \\
\hline LL & Log likelihood \\
\hline LLETZ & Large loop excision of the transformation zone \\
\hline LSIL & Low grade squamous intraepithelial lesion \\
\hline OR & Odds ratio \\
\hline PAMP & Pathogen associated molecular pattern \\
\hline PAP & Papanicolaou test \\
\hline PMBC & Peripheral blood mononuclear cell \\
\hline PPV & Positive predictive value \\
\hline PROBE & Prospective specimen collection, retrospective blinded evaluation \\
\hline PRR & Pattern recognition receptor \\
\hline
\end{tabular}




$\begin{array}{ll}\text { NF-kB } & \text { Nuclear factor kappa B } \\ \text { NK } & \text { Natural killer } \\ \text { NPV } & \text { Negative predictive value } \\ \text { RAND 36 } & \text { Research and Development 36 item Health Survey } \\ \text { Rb } & \text { Retinoblastoma } \\ \text { RCT } & \text { Randomized controlled trial } \\ \text { RR } & \text { Relative risk } \\ \text { RCT } & \text { Randomized controlled trial } \\ \text { SC] } & \text { Squamocolumnar junction } \\ \text { SD } & \text { Standard deviation } \\ \text { SSO } & \text { Sequence specific oligonucleotide } \\ \text { Th cells } & \text { Thelper cells } \\ \text { TLR } & \text { Toll like receptor } \\ \text { TNF } & \text { Tumor necrosis factor } \\ \text { Tregs } & \text { Regulatory T cells } \\ \text { TOPIC } & \text { Topical imiquimod treatment of high-grade CIN } \\ \text { VAIN } & \text { Vaginal intraepithelial neoplasia } \\ \text { VIN } & \text { Vulvar intraepithelial neoplasia } \\ \text { VLP } & \text { Virus-like particle }\end{array}$




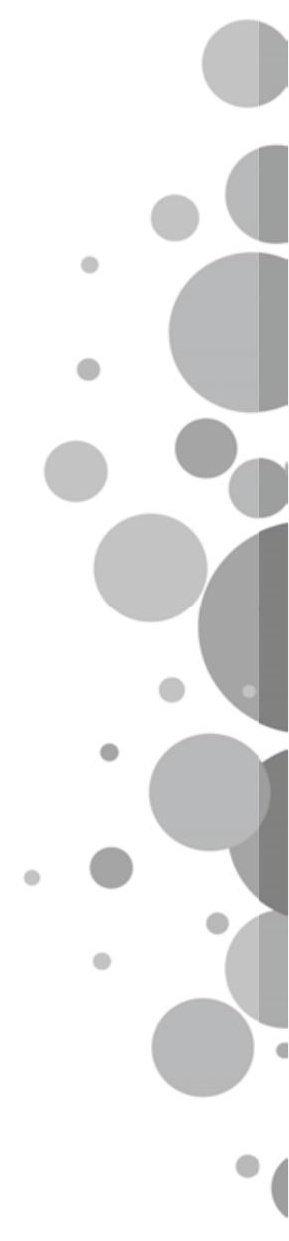




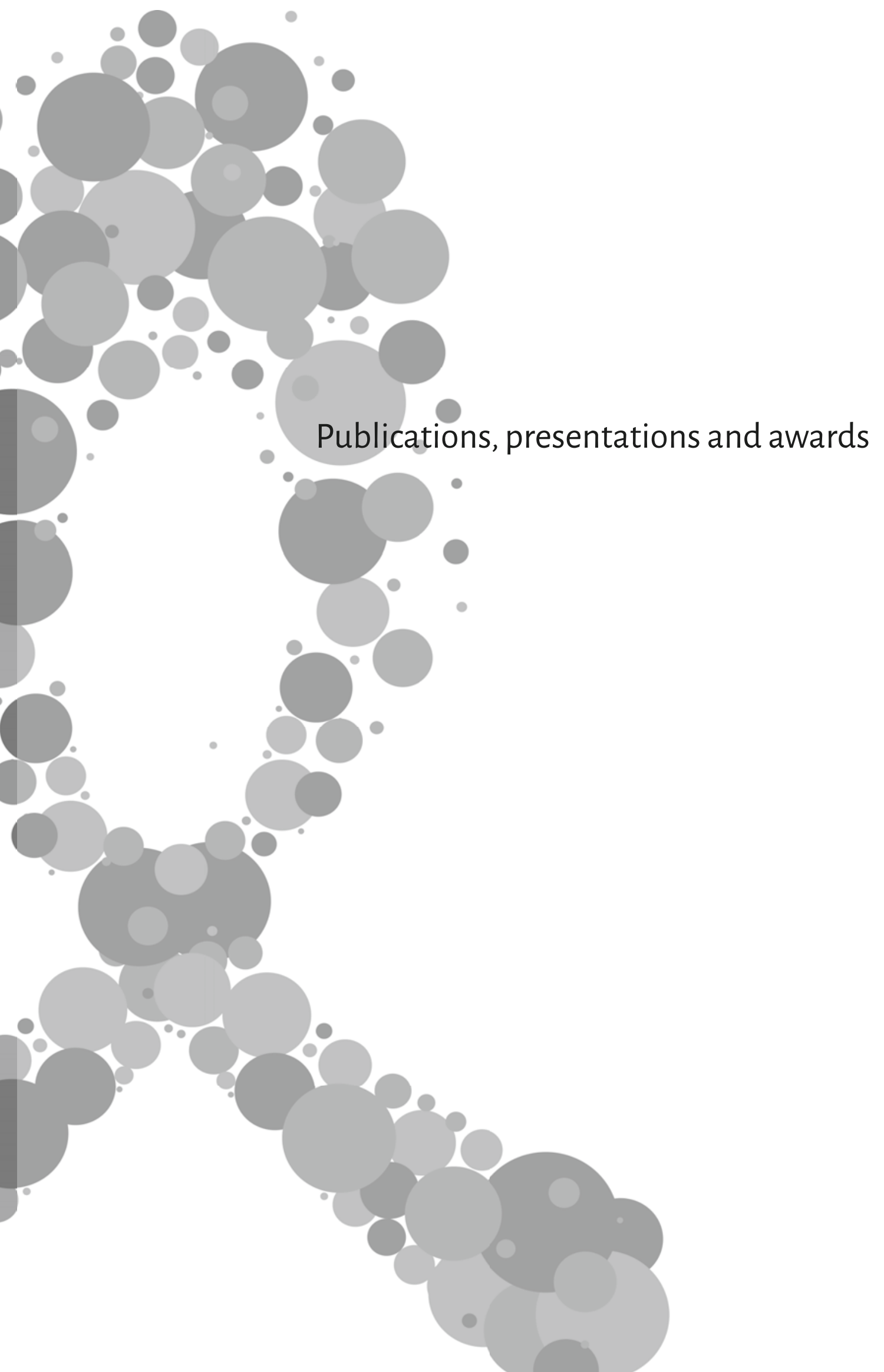




\section{Publications}

Koeneman MM, Hendriks N, Kooreman LFS, Winkens B, Kruitwagen RFPM, Kruse A]. Smoking status and parity are associated with spontaneous regression of high-risk HPV-positive CIN2. Submitted

Koeneman MM, Ovestad IT, Schellekens ], Munk AC, Janssen EAM, Tilanus MG], Kruitwagen RFPM, Kruse A]. A common epitope in HLA-DRB1*13/14 may protect against HPV16 related highgrade cervical intraepithelial neoplasia. Submitted

Koeneman MM, Ovestad IT, Janssen EAM, Ummelen M, Kruitwagen RFPM, Hopman AH, Krüse A]. Gain of chromosomal region 3926 as a prognostic biomarker for high-grade cervical intraepithelial neoplasia: literature overview and pilot study.

Pathology \& Oncology Research. 2018 Oct 25

van de Sande AJM, Koeneman MM, Gerestein CG, Kruse A], van Kemenade F], van Beekhuizen H]. TOPical Imiquimod treatment of residual or recurrent cervical intraepithelial neoplasia (TOPIC-2 trial): a study protocol for a randomized controlled trial.BMC Cancer. 2018 ]un 15;18(1):655.

Koeneman MM, Essers BA, Gerestein CG, van de Sande AJM, Litjens RJNTM, Boskamp D, Coossens MF], Beekhuizen H], Kruitwagen RFPM, Kruse A], Dirksen CD. Treatment of Cervical Intraepithelial Neoplasia: Patients Preferences for Surgery or Immunotherapy with Imiquimod. Journal of Immunotherapy. 2017 Feb; 40 (4): 148-153.

Koeneman MM, Kruse A], Kooreman LF, Zur Hausen A, Hopman AH, Sep S], Van Corp T, Slangen BF, van Beekhuizen H], van de Sande A], Gerestein CG, Nijman HW, Kruitwagen RF. Preliminary stop of the TOPical Imiquimod treatment of high-grade Cervical intraepithelial neoplasia (TOPIC) trial. BMC Cancer. 2017 Feb 7;17(1):110.

Koeneman MM, van Lint FH, van Kuijk SM, Smits L], Kooreman LF, Kruitwagen RF, Kruse A]. A prediction model for spontaneous regression of cervical intraepithelial neoplasia grade 2, based on simple clinical parameters. Hum Pathol. 2016 Sep 30.

Koeneman MM, Heiligers-Duckers C, Van der Velde R, Wouda S, De Rooij MJM, Janssen MJW, Boskamp D. Ovarian Leydig cell hyperplasia as a rare cause of hair loss in a postmenopausal female patient: a case report and diagnostic approach towards postmenopausal hyperandrogenism. Eur] Obstet Cynecol Reprod Biol. 2016 Apr;199:198-200

Koeneman MM, Kruse A], Kooreman LF, Zur Hausen A, Hopman AH, Sep S], Van Corp T, Slangen BF, van Beekhuizen H], van de Sande M, Gerestein CG, Nijman HW, Kruitwagen RF.TOPical Imiquimod treatment of high-grade Cervical intraepithelial neoplasia (TOPIC trial): study protocol for a randomized controlled trial. BMC Cancer. 2016 Feb 20;16(1):132 
Koeneman MM, Kruitwagen RFPM, Kruse A]. Clinical applicability of immunotherapy in cervical intraepithelial neoplasia. World ] Obstet Gynecol 2016 February 10; 5(1): 1-4

Koeneman MM, van de Sande A], van Beekhuizen H], Gerestein KC, van de Laar R, Kruitwagen RF, Kruse A]. Physicians' Awareness, Attitudes, and Experiences Regarding Imiquimod Treatment of Vaginal and Cervical Intraepithelial Neoplasia. J Low Cenit Tract Dis. 2016 Jan;20(1):75-9.

De Witte C], van de Sande A], van Beekhuizen H], Koeneman MM, Kruse A], Cerestein CC. Imiquimod in cervical, vaginal and vulvar intraepithelial neoplasia: a review.

Gynecol Oncol. 2015 Nov;139(2):377-84

Koeneman MM, Kruitwagen RF, Nijman HW, Slangen BF, Van Corp T, Kruse A]. Natural history of high-grade cervical intraepithelial neoplasia: a review of prognostic biomarkers.

Expert Rev Mol Diagn. 2015 Apr;15(4):527-46.

Kremer ME, Dirix M, Koeneman MM, van Baren R, Heij HA, Wijnen MH, Wijnen RM, van der Zee DC, van Heurn LW. Quality of life in adulthood after resection of a sacrococcygeal teratoma in childhood: a Dutch multicentre study. Arch Dis Child Fetal Neonatal Ed. 2015 May;100(3):F229-32

Kremer ME, Koeneman MM, Derikx JP, Coumans A, van Baren R, Heij HA, Wijnen MH, Wijnen RM, van der Zee DC, van Heurn EL. Evaluation of pregnancy and delivery in 13 women who underwent resection of a sacrococcygeal teratoma during early childhood.

BMC Pregnancy Childbirth. 2014 Dec 12;14(1):407.

Koeneman MM, Kruse A], Sep S], Gubbels CS, Slangen BF, van Gorp T, Lopes A, Gomez-Garcia E, Kruitwagen RF. A family history questionnaire improves detection of women at risk for hereditary gynecologic cancer: a pilot study. Fam Cancer. 2014 Sep;13(3):469-75.

Koeneman MM, Koek CH, Bemelmans M, Peeters LL. Perihepatic adhesions: an unusual complication of hemolysis, elevated liver enzymes and low platelet syndrome.

World ] Castroenterol. 2014 Jul 14;20(26):8726-8.

Koeneman MM, De Graaff AA, Van Beek J], Zipp-Haest K. Een bijzondere ectopische graviditeit. NTOG. 2014 juli, vol 127: 287-292

Debats IB, Koeneman MM, Booi DI, Bekers O, van der Hulst RR.Intravenous arginine and human skin graft donor site healing: a randomized controlled trial.

Burns. 2011 May;37(3):420-6. 


\section{Presentations}

Koeneman MM, Ovestad IT, Janssen EAM, Ummelen M, Kruitwagen RFPM, Hopman AH, Krüse A]. Gain of 3926 as a prognostic biomarker for high-grade cervical intraepithelial neoplasia. ESCO conference, Vienna, Austria, 4-7 November 2017 [poster]

MM Koeneman et al. What women want: voorkeuren van vrouwen in de behandeling van hooggradige $\mathrm{CIN}$ laesies met lisexcisie of imiquimod crème.

Gynaecongres. Apeldoorn, the Netherlands, 17-18 November 2017.

[oral presentation, $1^{\text {st }}$ prize Wim Schellekens Award]

MM Koeneman, FHM van Lint, SM] van Kuijk, LJM Smits, LFS Kooreman, RFPM Kruitwagen, A] Kruse. A prediction model for spontaneous regression of cervical intraepithelial neoplasia grade 2 , based on simple clinical parameters.

$16^{\text {th }}$ biennial meeting of the ICCS, Lisbon, Portugal, 29-31 October 2016. [poster]

MM Koeneman, BA Essers, CG Gerestein, AJM van de Sande, RJNTM Litjens, D Boskamp, MF] Coossens, $\mathrm{H}$ ] Beekhuizen, RFPM Kruitwagen, AJ Kruse, CD Dirksen. Womens' preferences for surgery or imiquimod as treatment for high-grade cervical intraepithelial neoplasia: a discrete choice experiment.

$16^{\text {th }}$ biennial meeting of the ICCS, Lisbon, Portugal, 29-31 October 2016. [poster]

MM Koeneman, A.J. Kruse, E. Comez-Garcia, C.S. Cubbels, S. Sep, B. Slangen, T. van Corp, R. Kruitwagen. Routine use of a screening questionnaire improves detection of individuals that may be at risk of hereditary cancer. ESCO conference. Liverpool, UK, October 2013. [poster]

MM Koeneman, A.J. Kruse, E. Comez-Carcia, C.S. Cubbels, S. Sep, B. Slangen, H. van Beekhuizen, T. van Gorp, R. Kruitwagen. Routine use of a screening questionnaire improves detection of individuals that may be at risk of hereditary cancer. Tenth Annual AACR International Conference on Frontiers in Cancer Prevention Research, Boston, USA, 22-25 October 2011. [poster]

\section{Awards}

\section{$\mathbf{1}^{\text {st }}$ prize Wim Schellekens Award}

Oral presentation at Cynaecongres, Apeldoorn, the Netherlands, 17-18 November 2017.

MM Koeneman et al. What women want: voorkeuren van vrouwen in de behandeling van hooggradige $\mathrm{CIN}$ laesies met lisexcisie of imiquimod crème. 



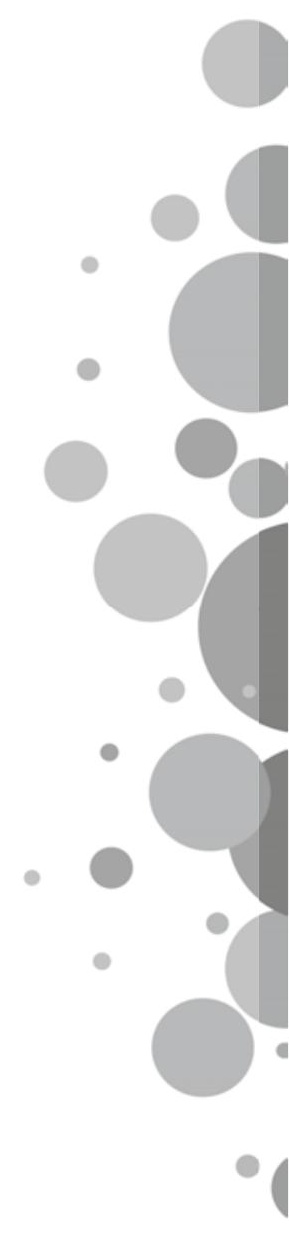




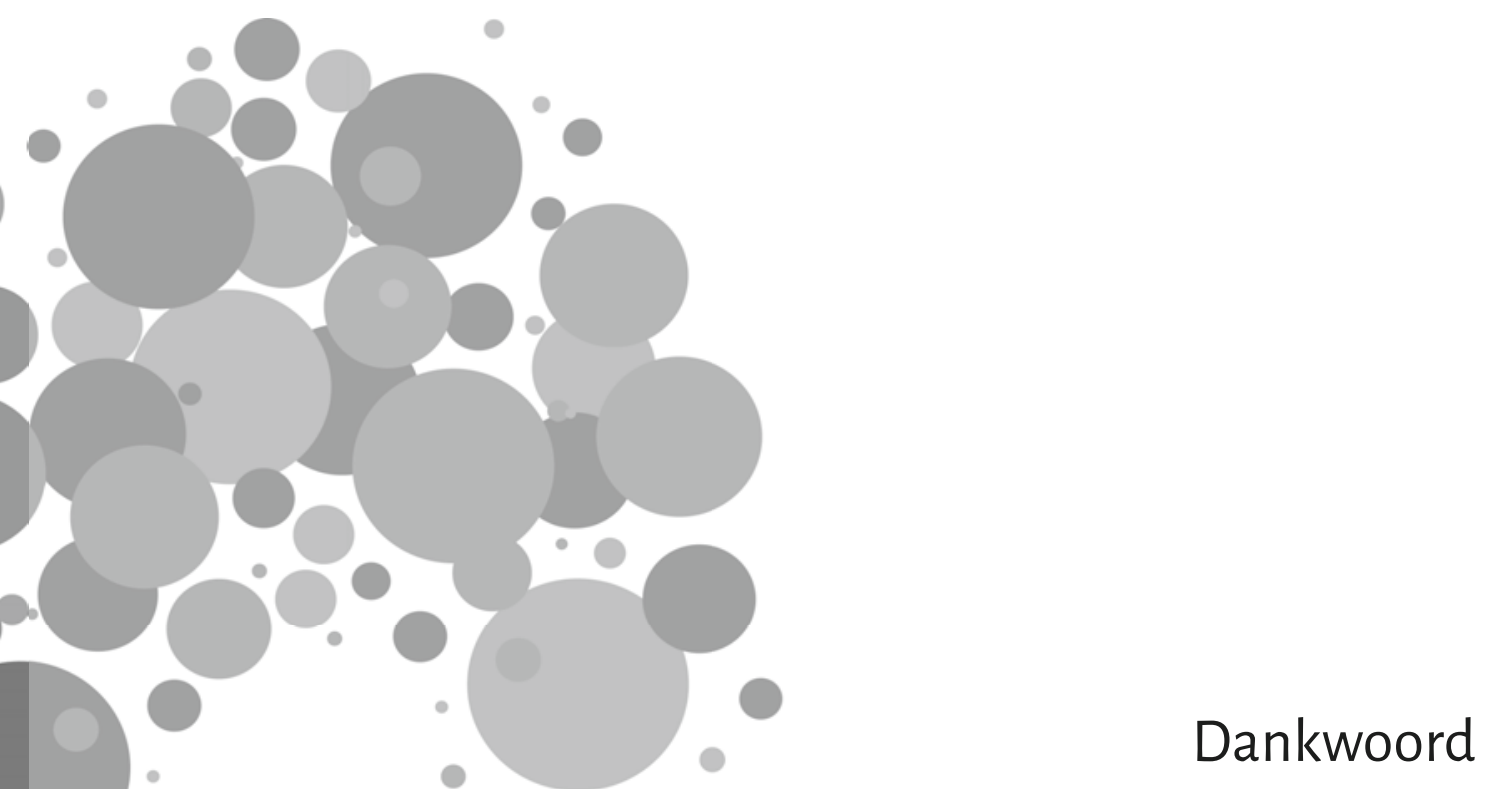


Allereerst natuurlijk heel veel dank aan de $\mathbf{1 2 0}$ vrouwen die deelnemen aan de TOPIC3 studie. Deelname aan wetenschappelijk onderzoek is niet vanzelfsprekend!

Professor Kruitwagen, u hebt mijn beeld van een professor als verstrooide grijze man volledig veranderd. Ik bewonder uw enorme toewijding aan de patiëntenzorg én het onderzoek, uw flexibiliteit, uw betrokkenheid bij de AIOS en onderzoeker als persoon, maar ook uw snelheid: simpele vragen worden vrijwel direct beantwoord en op de revisie van een manuscript hoefde ik zelden langer dan een paar dagen te wachten. Veel dank voor de kansen die u mij gegeven heeft, in het onderzoek én de kliniek!

Professor Nijman, mijn promotor op afstand. Hartelijk dank voor uw hulp bij de opzet van de TOPIC studie. We hebben nog een interessant onderzoeksproject lopen, ik kijk uit naar de resultaten!

Mijn copromotor, Arnold. Het eerste project dat we samen ondernamen was het familieanamnese-onderzoek. Ik was keuzeco en wilde gynaecoloog worden, dus moest er onderzoek gedaan worden. Het bleek een succes en ik mocht blijven. Jij had net een grant gekregen voor de imiquimod-studie en daar mocht ik mee aan de slag. Zo begonnen we aan ons geneesmiddelenonderzoek-avontuur, waarmee we allebei geen ervaring hadden. De METCaanvraag duurde meer dan een jaar, waarin ik me echt wel eens heb afgevraagd of het überhaupt goed zou komen. Maar dat kwam het, zo blijkt. Bedankt voor je geweldige begeleiding: nooit bemoeizuchtig of veeleisend, maar sturend en ondersteunend. Je liet me vrij en vertrouwde me, maar wist me op de juiste momenten bij te sturen. Ik heb veel van je geleerd op het gebied van 'schrijven' en dat is me heel waardevol! Daarnaast was je niet alleen betrokken bij mijn onderzoek, maar ook bij mijzelf, Bob en Stijn. Soms belde je gewoon om te vragen hoe het allemaal ging en vroeg je of je nog iets voor me kon doen, geweldig! Ik hoop dat we blijven samenwerken aan het TOPIC project en dat we nu eindelijk eens een datum gaan prikken voor die BBQ in Zwolle (we kunnen ook naar de Librije overigens)!

Ik wil de beoordelingscommissie en promotiecommissie bedanken voor het beoordelen van mijn proefschrift en de oppositie tijdens mijn promotie.

Veel dank aan het stafconventbestuur van het MUMC, voor het gestelde vertrouwen met het toekennen van een bijdrage voor onze studie uit het academisch fonds.

Ook wil ik Clemens van Ede bedanken, onze contactpersoon bij MedaPharma, voor de constructieve samenwerking en financiële ondersteuning van onze studie.

Het TOPIC-team: Heleen van Beekhuizen, Meike van de Sande, Kees Gerestein en Jurgen Piek, hartelijk dank voor de fijne samenwerking, jullie enthousiasme en jullie inspanningen om zoveel vrouwen te includeren. Meike, wij bespraken aan de telefoon ook altijd even hoe het met 'de rest van ons leven' ging, dat vond ik altijd heel gezellig - zeker toen we bijna tegelijk moeder werden! 
Een speciaal woord van dank aan het oncoteam van het MUMC: Roy, Toon, Brigitte, Sandrijne en sinds kort ook Erica. Wat zijn jullie een geweldig team: kundig, betrokken, laagdrempelig en gezellig, mijn onco-diff is een feest! Ik wil Brigitte in het bijzonder bedanken voor haar betrokkenheid bij de TOPIC3 studie, na het vertrek van Arnold uit Maastricht. Fijn dat we op je kunnen bouwen! Ook veel dank aan Petra op den Oordt voor de secretariële ondersteuning, je bent geweldig en onmisbaar!

Mijn steun en toeverlaat op de werkvloer: Charlotte Penders, onze verpleegkundig specialist! Zonder jou waren de inclusies nooit zo vlot gegaan. Je enthousiasme, betrokkenheid en doortastendheid was heel belangrijk voor de werving en begeleiding van patiënten - $i k$ hoop dat ik je dat vaak genoeg heb gezegd! Je bent een held! Ook een groot dankwoord voor Moniek Kamps, die alle controle uitstrijkjes doet en daarbij mijn vragenlijsten uitdeelt en inneemt. Ik weet dat het soms een hele klus is en dat je dat allemaal 'extra' doet voor mij, dank je wel! Ook een speciaal woord van dank aan Helma van Berkel, research nurse in het Catharina Ziekenhuis, voor je ondersteuning van de studie. Je bent kundig, snel en doortastend!

Mijn 'opvolger', Natasja Hendriks: wat geweldig dat jij er ineens was om de TOPIC3 van mij over te nemen! En wat heb je het geweldig opgepakt! Binnen no-time had je het hele onderzoek onder controle en rondde je de inclusies 4 maanden eerder af dan verwacht. We gaan dit project samen afronden, leuk!

Ik ben ook veel dank verschuldigd aan mijn medeauteurs. Loes Kooreman, wat was ik zonder jouw inzet op pathologie-gebied. Dank voor al je hulp en inspanningen, hopelijk bereik jij dit punt ook snel! Irene Ovestad, Ane Cecilie Munk and Emiel Janssen: our research partners from Norway, thank you for a constructive and pleasant cooperation! En ook veel dank aan mijn andere coauteurs, voor een fijne en leerzame samenwerking: Ton Hopman, Monique Ummelen, Jennifer Schellekens, professor Tilanus, Freyja van Lint, Sander van Kuijk, Luc Smits, Björn Winkens, professor Zur Hausen, professor Dirksen, Brigitte Essers, Rogier Litjens, Dieuwke Boskamp, Medi Coossens en Simone Sep.

Een bijzonder woord van dank aan de gynaecologen van het VieCuri. Jullie namen me aan als ANIOS, zonder ook maar enige ervaring - dat vind ik heel bijzonder! Dankzij jullie had ik een vliegende start en ben ik gekomen waar ik nu ben als gynaecoloog in opleiding. Ik heb me ontzettend thuis gevoeld bij jullie! Beste Iris, Petra, Dieuwke, Anika, Annemieke, Erik, Ella, Rafli, maar ook de inmiddels gepensioneerde Hans van Beek en Robert Jan Iding: bedankt voor jullie vertrouwen en leerschool!

Bazen van het MUMC, waar ik het $2^{\mathrm{e}}$ en $3^{\mathrm{e}}$ jaar van mijn opleiding deed en nu mijn differentiatie oncologie: bedankt voor de fijne leeromgeving, het persoonlijk contact en de leuke momenten buiten het ziekenhuis (de skireis, carnaval, bruiloften, etc). 
Collega AIOS in het MUMC en het VieCuri, waar zijn we zonder elkaar! Bedankt voor de gezellige momenten in de assistentenkamer, het sparren over medisch inhoudelijke vraagstukken, maar ook voor de opvang en steun in mindere tijden en het begrip dat je niet meer zo flexibel kunt zijn als je zwanger bent of een klein kind hebt. Jullie zijn goud waard!

Lieve Kim, Lotte, Soetinah en Frouke, Venloose meisjes van het eerste uur! Wat was het heerlijk om met jullie mijn carrière in de gynaecologie te beginnen! Bedankt voor de vele uren in de auto met de carpoolmeisjes, waarin we de dagen in het ziekenhuis doorspraken, even konden 'afschakelen', maar vooral elkaar heel goed leerden kennen! Bedankt voor de vele gezellige lunches in het ziekenhuis en natuurlijk de borreltjes met het Zondag-op-Donderdag clubje, toen Lotte ook in Maastricht woonde. Jullie zijn de constante collega-factor, ik hoop dat dat zo blijft!

Meisjes van damesjaarclub Mystique! Wat geweldig dat we elkaar na 15 jaar nog steeds zo regelmatig zien. We gaan met onze jaren mee: van een mooie studententijd op de kroeg naar de mama-app, waarin ik heel veel goede adviezen van jullie krijg, maar vooral ook heel veel schattige fotootjes kan bekijken (en sturen). In het bijzonder nog een woordje voor Sarah en Chantal, met wie ik de afgelopen jaren trouw bleef jaarclubeten in Maastricht. Heerlijk om jullie zo regelmatig te spreken over lief en leed op ons werk, in het onderzoek, zwangerschappen, kids en onze liefdes. Ik zal het missen!

Disputatio ad Mosam Quadripara,mijn dispuut: bedankt voor mijn geweldige studententijd en de blijvende vriendschappen, maar ook voor het voorbeeld dat de ouderejaars voor mij waren: door jullie werd ik geïnspireerd en geadviseerd om onderzoek te gaan doen naast mijn studie. In het bijzonder wil ik bedanken Aron (en Anneke), Margit, Jonas (en Anne), Tim, Tom, Emiel, Sanne, Rosan, Marleen en Imke. Aron en Anneke, op alle gebieden gaan we gaan samen op: studeren, promoveren, specialiseren, maar ook reizen maken en kinderen krijgen. Bedankt voor alle mooie herinneringen in Maastricht en Eindhoven, en hopelijk straks in Ede en verder! Margit, Gitteke, wat hebben we al een hoop meegemaakt! Ook al zien we elkaar niet veel, we kunnen altijd op elkaar terug vallen - je bent me dierbaar! Jonas en Anne: ons spiegelbeeld qua beroepen; het is leuk om zo gelijk op te gaan (met zelfs maar 4 dagen tussen onze kindjes). Hopelijk volgen er nog vele malen vastelaovend! Tim, Tom en Emiel: lichting 2004, need I say more? Rosan, ook al ben je nu even op afstand (zeg maar letterlijk aan de andere kant van de wereld), bedankt voor alle fijne gesprekken en momenten! Sanne, Marleen en Imke, de Afrika-meisjes en mede-dokters, ik hoop op nog vele gezellige borreltjes en etentjes.

Maarten en Veerle, lievevrienden! Als'koudekant'werd ikgelukkigal heel snel opgenomen injullie hechte vriendschap met Bob. Ik heb mooie herinneringen aan onze tijd in Maastricht, zoals de uitde-hand-gelopen borrel bij Thembi, het skiweekend en natuurlijk aan pannenkoekenrestaurant de Clown! Sinds jullie verhuizing en onze kinderen zijn de bezoekjes aan elkaar schaarser, maar des te waardevoller. Ik hoop op nog vele oud-en-nieuws, etentjes en kinderuitjes; en in de tussentijd is er gelukkig altijd de app voor de nodige updates! 
Frouke en Janneke, mijn paranimfen. Frouke, van collega en carpoolmaatje tot geweldige vriendin. We delen lief en leed, in het groot en klein: van de geboorte van jouw dochter tot een appje over wat we die avond gaan eten. Ik heb bewondering voor jouw vermogen om alle ballen in de lucht te houden én betrokken te blijven bij de mensen om je heen. Ik ben blij dat jij vandaag naast mij staat en ik hoop dat dat altijd zo blijft! Janneke, het begon allemaal bij het overdrachtsbestand waarin ik aan het 'opruimen' was, toen onze eerste dienst samen begon. Je zei: 'met jou kan ik lezen en schrijven' en dat bleek ook zo! Bedankt voor de vele koppen thee op jouw kamer, de wandelingen op de Pietersberg en onze fietstochtjes! Ook heel veel dank voor het doorlezen van mijn héle proefschrift, waaruitje nog vele schoonheidsfoutjes hebt weten te halen. Stijn-en wij-hopen dat je ook in Ede wil komen oppassen!

Frank en Liesbeth, mijn lieve schoonouders, bedankt voor jullie eindeloze interesse en betrokkenheid bij ons en sinds kort ook bij onze zoon: wat zijn jullie een fijne opa en oma! Wouter en Alice, mijn schoonbroer en -zus. Wat bijzonder dat we in hetzelfde jaar een zoon kregen. Ik kijk uit naar de stappen die ze samen gaan zetten, te beginnen met hun eerste wintersport!

Lieve Lisette en Pauline, mijn zus en zusje. Vanuit ons ouderlijk huis gingen we allemaal onze eigen weg: studeren in Utrecht, Maastricht en Enschede. Lis, als mede-dokter konden we veel ervaringen delen en begreep jij altijd goed welke hobbels ik tegenkwam in de combinatie opleiding/onderzoek. Inmiddels heb jij de zorgsector verlaten; ik denk dat je daarmee een heel goede keuze hebt gemaakt. Sinds de geboorte van Stijn is ons contact intensiever geworden, daar ben ik blij mee! Bedankt voor je luisterend oor en leuke verhalen! Pien, nadat je op de middelbare school mijn kleine zusje was, ging je in je studententijd je eigen weg. We deelden het enthousiasme over het studentenleven, de studentenvereniging en een bestuursjaar; ik ben trots op hoe je je in die tijd ontwikkeld hebt! Je bent een fijne stabiele factor, we hebben aan weinig woorden genoeg. Ik ben blij dat ik binnenkort wat dichter bij jullie kom wonen!

Papa en mama, bedankt voor alle kansen die ik van jullie kreeg: het 'proefklasje' van het tweetalig VWO, studeren en op kamers in Maastricht en de ruimte om een bestuursjaar te doen, maar ook de mooie reizen die we als gezin hebben gemaakt! Het heeft me gemaakt wie ik ben en gezorgd dat ik nu hier sta. Sinds dit jaar zijn jullie behalve mijn ouders ook de grootouders van mijn zoon en dat geeft een nieuwe dimensie aan onze band! Mama, dank je wel voor het oppassen op de laatste maandagen die ik nodig had om mijn proefschrift af te maken. Binnenkort wonen we dichter bij jullie, daar kijk ik naar uit!

Lieve Bob! Jij bent mijn klankbord, steunpilaar en relativeringsvermogen, mijn reisgezel op de wereld en in het leven. Sinds kort ben je ook de vader van mijn zoon; en wat doe je dat geweldig! Ik ben zó blij met jou en zó trots op jou. Jouw eigen woorden hoor je nu terug: ik kan me de toekomst zonder jou niet voorstellen!

Stijn, kleine man! Wat waren we blij dat jij kwam. Je kwam in de afrondingsfase van mijn proefschrift, dus dat vroeg heel wat van mijn multitask-skills. Maar nu is het klaar en is maandag niet meer onderzoeksdag, maar mamadag! jij geeft elke dag een gouden randje... 


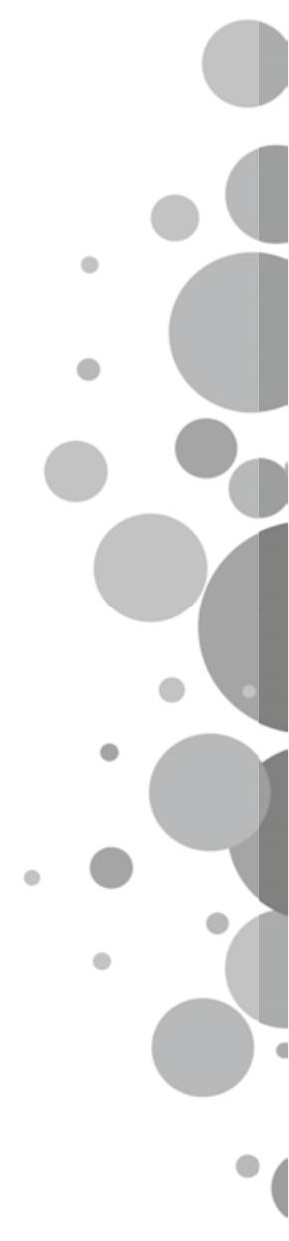



Margot Koeneman werd op 3 juni 1986 geboren in Eindhoven. In 2004 behaalde zij het gymnasiumdiploma aan het Kandinsky College te Nijmegen. Van 2004 tot 2011 studeerde zij geneeskunde aan de Universiteit Maastricht. $\mathrm{Na}$ het behalen van het artsexamen werkte zij van augustus 2011 tot maart 2013 als ANIOS gynaecologie in het VieCuri Medisch Centrum. In diezelfde periode startte zij met haar promotie onderzoek, onder begeleiding van Dr. A] Kruse en prof. dr. RFPM Kruitwagen. Sinds maart 2013 is zij daarnaast in opleiding tot gynaecoloog. De eerste vijf jaar van haar opleiding werden volbracht in het VieCuri Medisch Centrum (opleiders: dr. J] van Beek

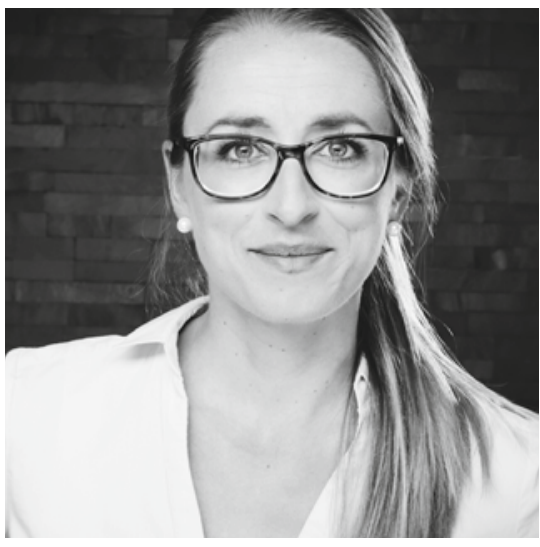
en dr. I van Cestel) en het MUMC (opleider: prof. dr. RFPM Kruitwagen). Het laatste deel van haar opleiding zal zij afmaken in het Ziekenhuis Gelderse Vallei te Ede (opleider: dr. MJN Weinans). Margot is in 2016 getrouwd met Bob Touwslager. Zij kregen in 2018 een zoon, Stijn. 




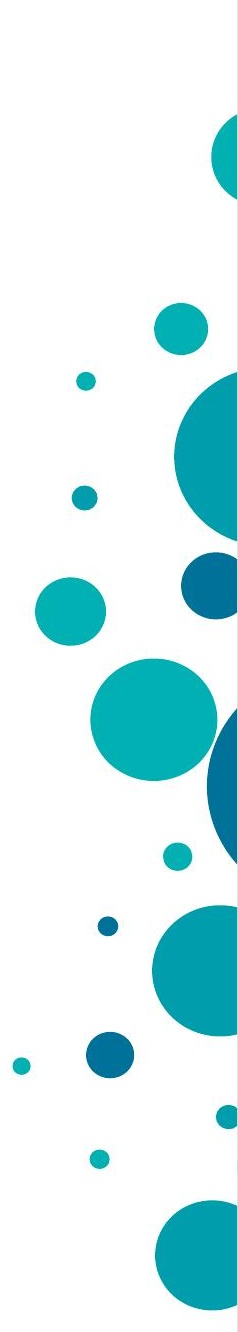

